Coastal Inlets Research Program

\title{
A Unified Sediment Transport Formulation for Coastal Inlet Application
}

Benoît Camenen and Magnus Larson

September 2007
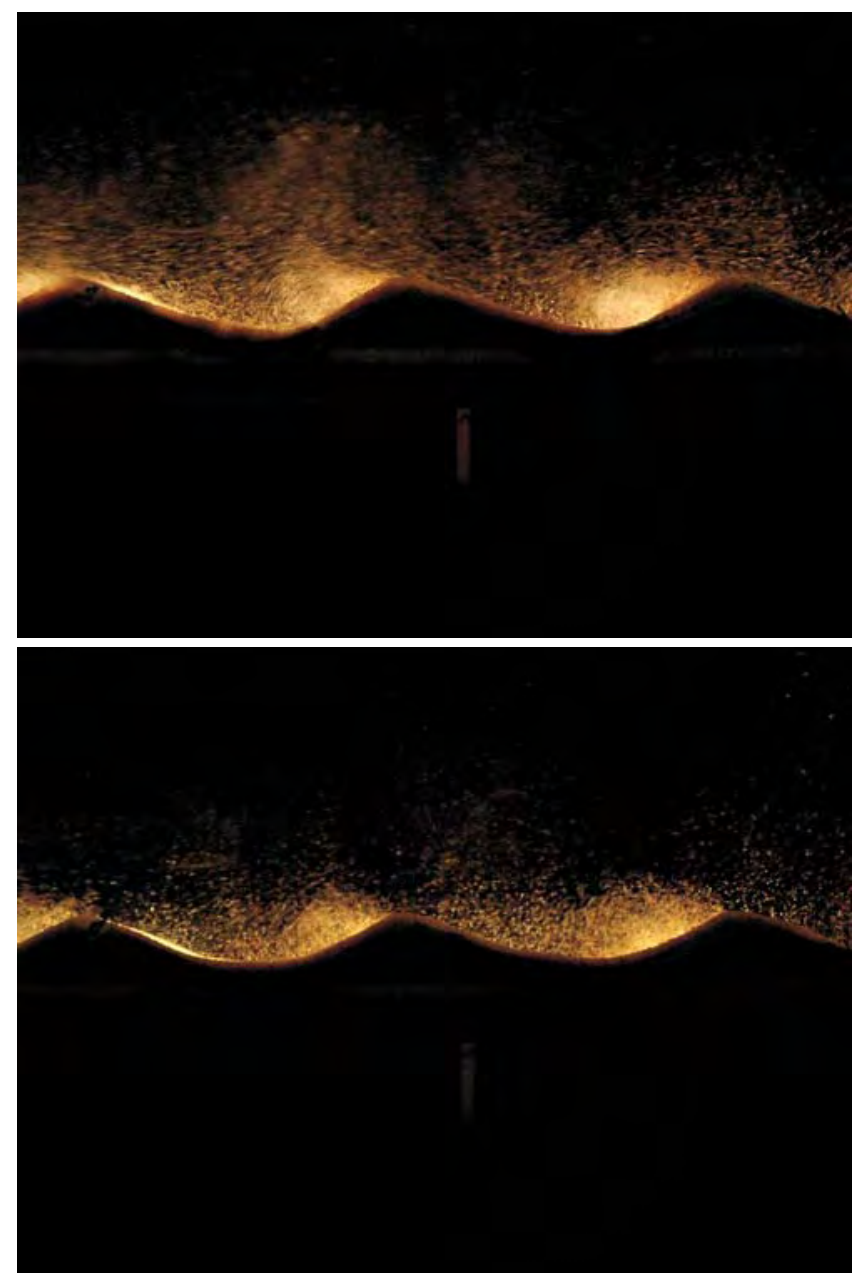

Sand suspended in vortices over a rippled bed by wave action in a laboratory tank. Photographs courtesy of Dr. Tomohiro Sekiguchii, taken at Osaka University, Japan. 


\title{
A Unified Sediment Transport Formulation for Coastal Inlet Application
}

\author{
Benoît Camenen \\ Cemagref Lyon \\ 3 Bis Quai Chauveau \\ CP 220, 69336 Lyon, CEDEX 09 \\ France \\ Magnus Larson \\ Department of Water Resources Engineering \\ Lund University \\ Box 118 \\ 22100 Lund \\ Sweden
}

Final report

Approved for public release; distribution is unlimited.

\author{
Prepared for Headquarters \\ U.S. Army Corps of Engineers \\ Washington, DC 20314-1000 \\ Under Coastal Inlets Research Program \\ Monitored by Coastal and Hydraulics Laboratory \\ U.S. Army Engineer Research and Development Center \\ 3909 Halls Ferry Road, Vicksburg, MS 39180-6199
}




\begin{abstract}
The Coastal Inlets Research Program (CIRP) is developing predictive numerical models for simulating the waves, currents, sediment transport, and morphology change at and around coastal inlets. Water motion at a coastal inlet is a combination of quasi-steady currents such as river flow, tidal current, wind-generated current, and seiching, and of oscillatory flows generated by surface waves. Waves can also create quasisteady currents, and the waves can be breaking or non-breaking, greatly changing potential for sediment transport. These flows act in arbitrary combinations with different magnitudes and directions to mobilize and transport sediment. Reliable prediction of morphology change requires accurate predictive formulas for sediment transport rates that smoothly match in the various regimes of water motion. This report describes results of a research effort conducted to develop unified sediment transport rate predictive formulas for application in the coastal inlet environment. The formulas were calibrated with a wide range of available measurements compiled from the laboratory and field and then implemented in the CIRP's Coastal Modeling System.
\end{abstract}

Emphasis of the study was on reliable predictions over a wide range of input conditions. All relevant physical processes were incorporated to obtain greatest generality, including: (1) bed load and suspended load, (2) waves and currents, (3) breaking and non-breaking waves, (4) bottom slope, (5) initiation of motion, (6) asymmetric wave velocity, and (7) arbitrary angle between waves and current. A large database on sediment transport measurements made in the laboratory and the field was compiled to test different aspects of the formulation over the widest possible range of conditions. Other phenomena or mechanisms may also be of importance, such as the phase lag between water and sediment motion or the influence of bed forms. Modifications to the general formulation are derived to take these phenomena into account. The performance of the new transport formulation was compared to several popular existing predictive formulas, and the new formulation yielded the overall best predictions among the formulas investigated. Results of this report are thus considered to represent a significant and operational step toward a unified formulation for sediment transport at coastal inlets and the nearshore where transport of non-cohesive sediment is common.

DISCLAIMER: The contents of this report are not to be used for advertising, publication, or promotional purposes. Citation of trade names does not constitute an official endorsement or approval of the use of such commercial products. All product names and trademarks cited are the property of their respective owners. The findings of this report are not to be construed as an official Department of the Army position unless so designated by other authorized documents. 


\section{Contents}

Figures and Tables......................................................................................................................

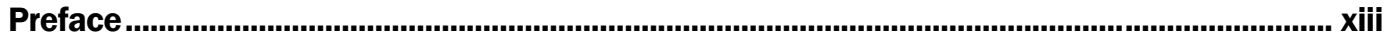

1 Introduction

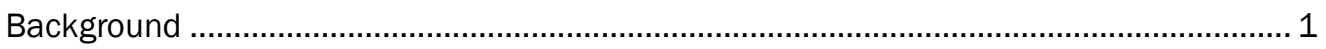

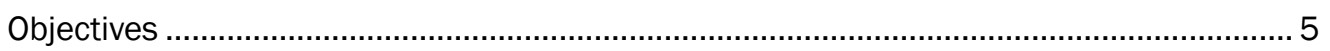

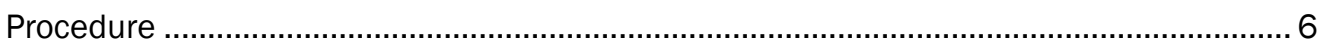

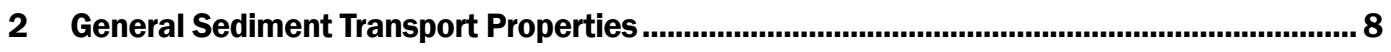

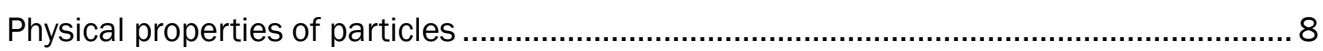

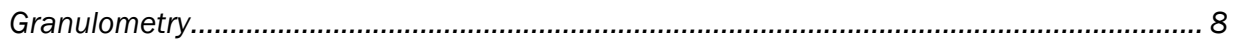

Porosity and friction angle .................................................................................... 9

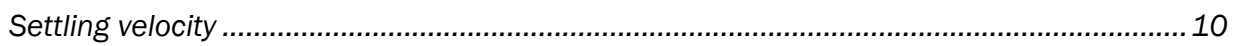

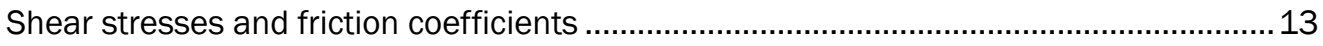

Bottom boundary layer flow..................................................................................... 13

Current-related shear stress..................................................................................... 14

Wave-related shear stress ....................................................................................... 15

Combined wave and current shear stress..................................................................17

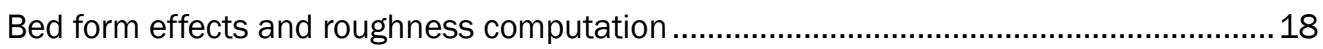

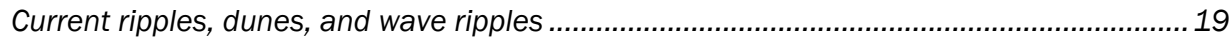

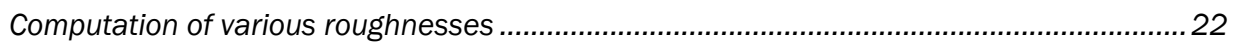

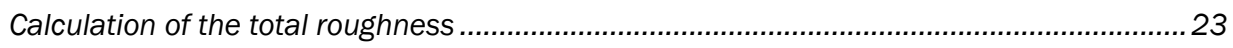

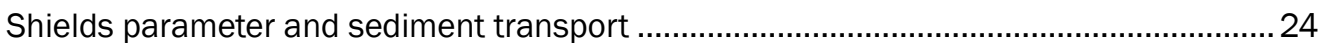

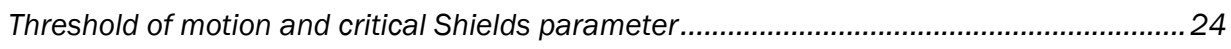

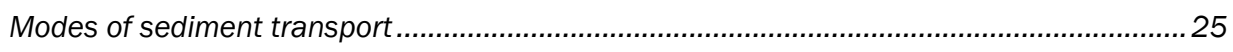

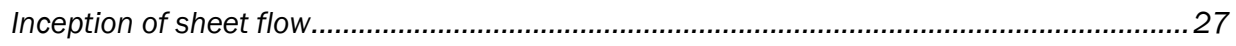

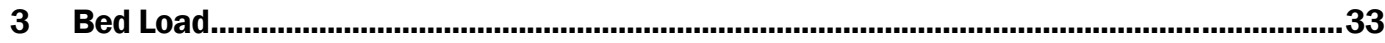

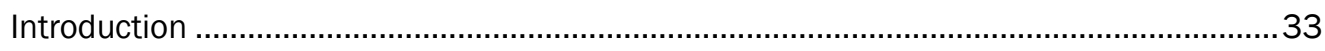

Previous studies on bed-load transport under wave and current interaction ....................34

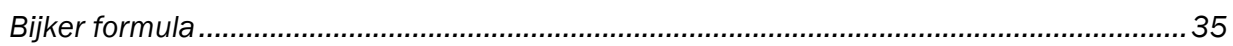

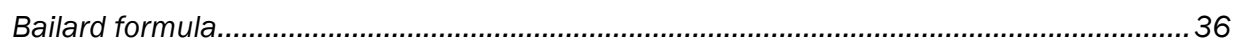

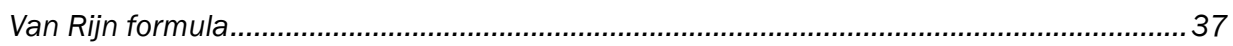

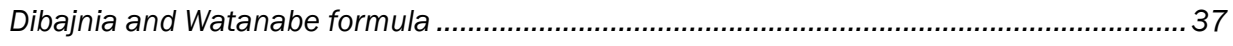

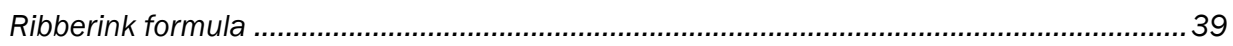

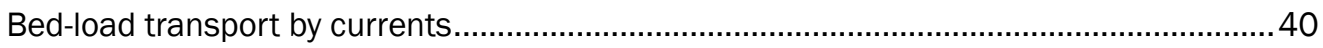

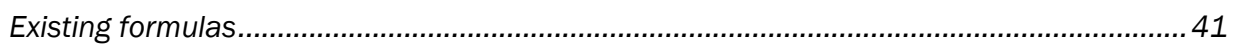

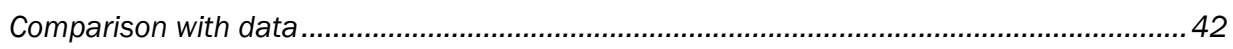

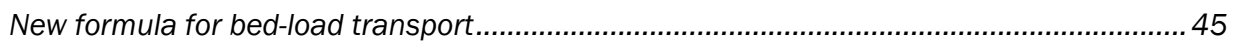

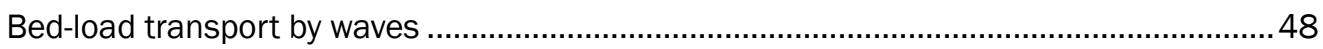

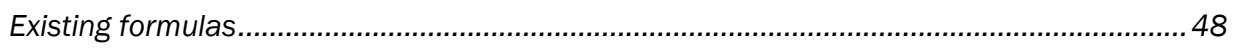

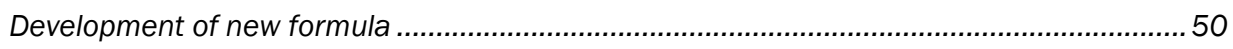

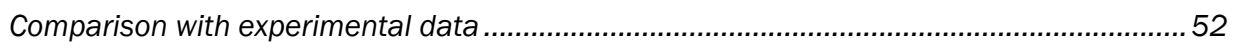




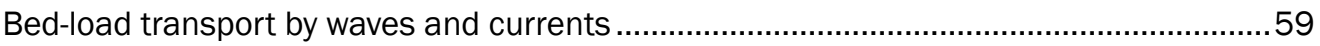

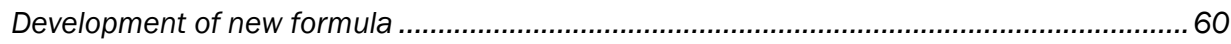

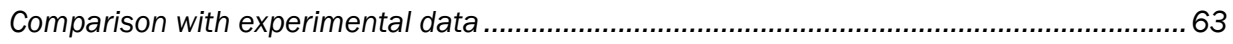

Comparison with existing formulas for waves and current .................................................6 65

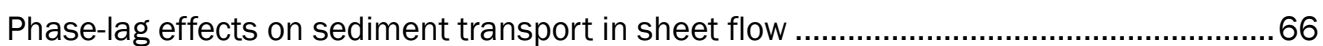

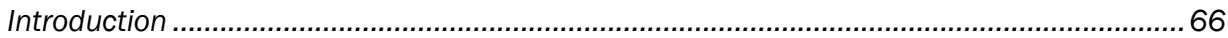

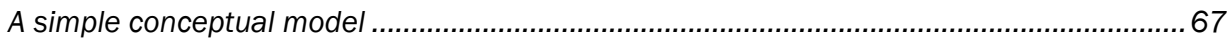

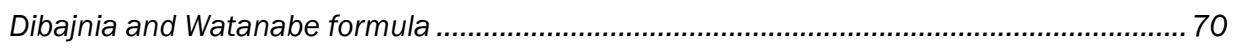

Modification of Camenen and Larson formula for phase lag.............................................. 71

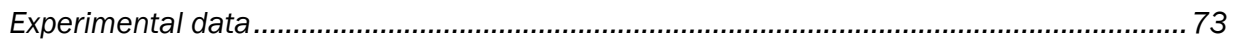

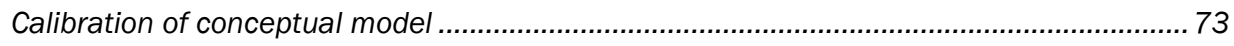

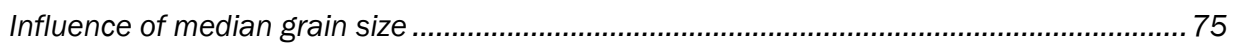

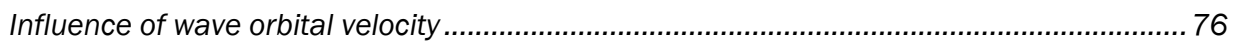

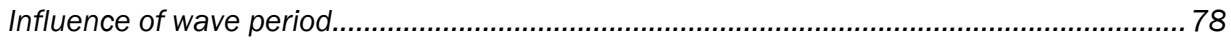

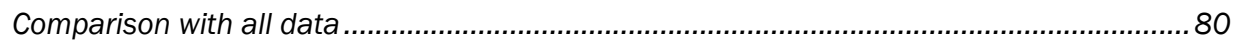

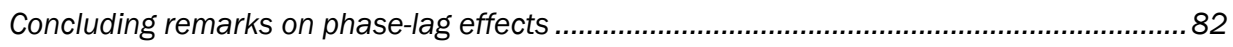

4 Suspended Load ........................................................................................................... 83

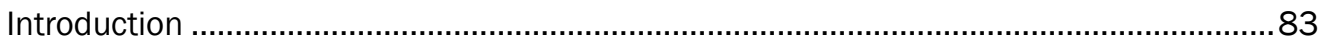

Equilibrium profile for suspended sediment................................................................. 86

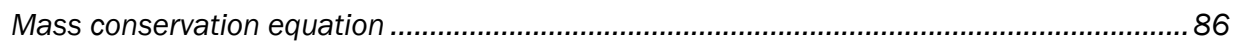

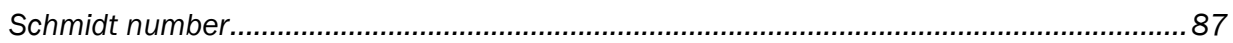

Sediment diffusivity and concentration profiles............................................................... 88

Sediment diffusivity due to steady current ............................................................. 91

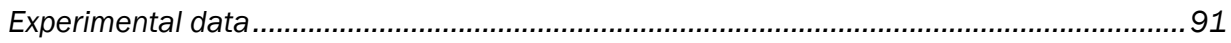

Shape of concentration profile.......................................................................................... 95

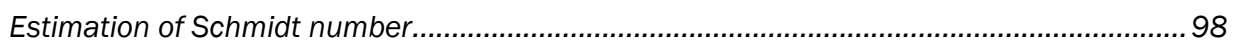

Sediment diffusivity in nonbreaking waves ..............................................................102

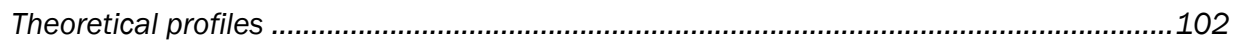

Estimation of sediment diffusivity profiles for oscillatory flows...........................................103

Starting point for suspended load..................................................................................... 108

Shape of concentration profile ......................................................................................... 112

Relationships for mean sediment diffusivity under waves .................................................118

New formula for mean sediment diffusivity due to waves................................................121

Interaction between waves and current ............................................................................ 124

Effect of breaking waves on sediment diffusivity ........................................................ 127

Extension of sediment diffusion expression ................................................................ 127

Experimental data with breaking waves ........................................................................130

Energy dissipation due to breaking waves ......................................................................130

Influence of Irribaren parameter and $u *_{w} / W_{s}$ on sediment diffusivity ...............................132

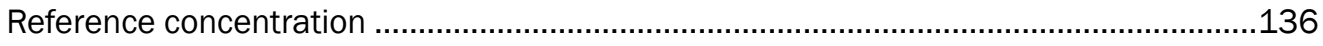

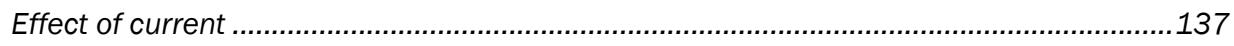

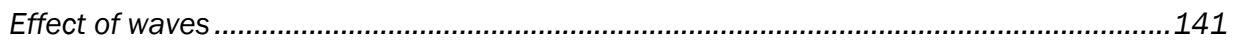

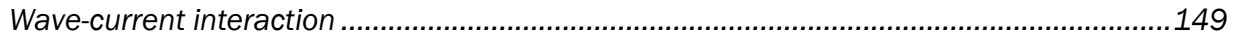

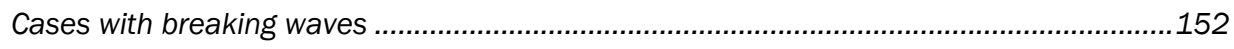

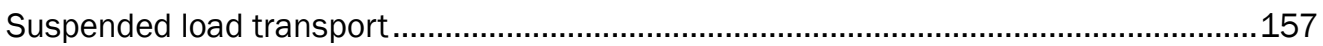

Existing formulas for suspended load under wave-current interaction ................................158 


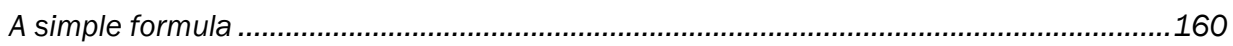

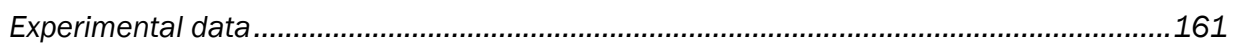

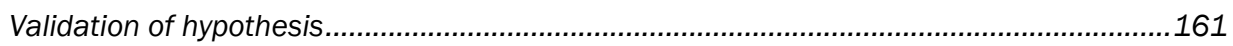

Comparison with experimental data in case of current only ...............................................167

Comparison with experimental data for wave-current interaction .........................................168

Suspended sediment transport for rippled beds .....................................................170

Effects of ripples on suspended load................................................................................ 171

Simple conceptual model for phase-lag effects on suspended load ...................................172

Modification of formula for asymmetric waves ....................................................................174

Observations of phase-lag effects on suspended load over ripples...................................175

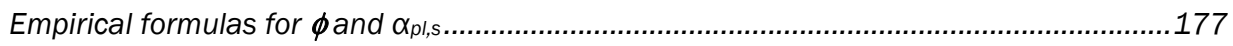

Sensitivity analysis for different formulas..................................................................... 181

Concluding remarks on phase-lag effects ................................................................185

5 A Unified Sediment Transport Formula for Coastal Inlet Application .............................. 186

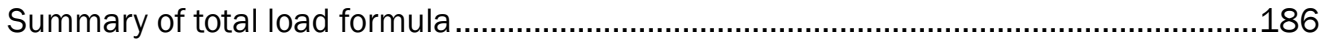

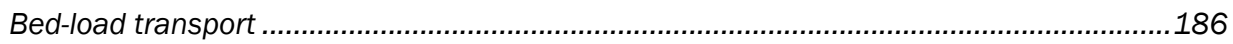

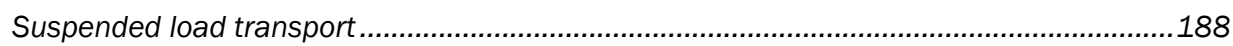

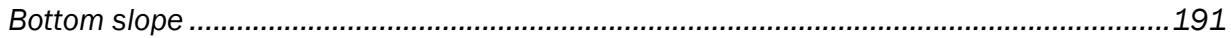

Velocity profiles for varying slope .................................................................................... 191

Application to coastal inlet studies .........................................................................193

Validation of longshore sediment transport ..................................................................... 193

Validation of cross-shore sediment transport ...................................................................201

Comments on morphological evolution using total load formulas.....................................205

6 Conclusions........................................................................................................................207

References..................................................................................................................................... 210

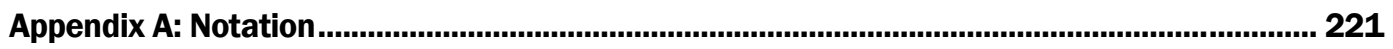

Appendix B: Computation of Mean Values for Onshore and Offshore Shields Parameter...... 229

\section{Report Documentation Page}




\section{Figures and Tables}

\section{Figures}

Figure 1. Hydrodynamic processes controlling sediment transport in an inlet environment (slide courtesy of N. C. Kraus)

Figure 2. Natural processes around an inlet for which predictions of sediment transport and morphological evolution are of importance (slide courtesy of N. C. Kraus)..................

Figure 3. Hydrodynamic forcing determining conditions for longshore sediment transport (after Soulsby 1997).

Figure 4. Sediment transport formulation. ………….......................................................... 5

Figure 5. Example of grain-size distribution (after Fredsøe and Deigaard 1994)............................. 9

Figure 6. Settling velocity for sediment with comparison of several formulas against measurements (for details on data and equations, see Camenen 2007).

Figure 7. Turbulent boundary layer structure with mean velocity profile.

Figure 8. Wave friction coefficients for plane bed predicted by different formulas.

Figure 9. Schematic diagram for non-linear interaction between wave and current bed shear stress (after Soulsby et al. 1993)

Figure 10. Schematic of ripples due to current (a) and waves (b)

Figure 11. Influence of grain size diameter (1), water depth (2), and wave orbital velocity (3); on bed-form predictions for current ripples (a), dunes (b), and wave ripples (c); and on roughness prediction according to different formulas.

Figure 12. Equivalent roughness ratio $k_{s} / d_{50}$ versus total Shields parameter $\theta$ for compiled data set together with predictions by studied formulas (for details on data and equations see Camenen et al. 2006).

Figure 13. Critical Shields parameter plotted against dimensionless grain size (equations for bed-load limit are from Van Rijn (1993) and Soulsby and Whitehouse (1997); equation for suspended load limit is from Van Rijn (1993)), compared to data with current (c) or waves (w) (for details on data see Hanson and Camenen 2007).

Figure 14. Modes of sediment transport (after Fredsøe and Deigaard 1994).

Figure 15. Schematic representation of different bed forms and sediment transport regimes for (a) increasing current, or (b) wave orbital velocity.

Figure 16. Classification of different types of sediment transport with respect to Shields parameter $\theta$ and ratio $U_{c} / W_{s}$ (after Shibayama and Horikawa 1982).

Figure 17. Comparison between observed critical wave orbital velocity $U_{w, c r, s f, \text { meas }}$ for inception of sheet flow and predicted value $U_{w, c r, s f, p r e d}$ using Equation 57.

Figure 18. Definition of (a) wave and current directions, and (b) horizontal time-dependent velocity variation at bottom in direction of wave propagation (after Dibajnia and Watanabe 1992).

Figure 19. Time variation of bottom velocity (a) in wave direction, and (b) induced shear stress for waves and current combined (after Ribberink 1998).

Figure 20. Distribution of median grain size and mean current speed for database compiled on sediment transport under steady current

Figure 21. Comparison between Meyer-Peter and Müller formula and compiled database on sediment transport rates. 
Figure 22. Effect of critical Shields parameter on bed-load transport rate: comparison between data and studied formulas.

Figure 23. Influence of critical Shields parameter on bed-load transport rate illustrated through data and Equation 81 (for different values on $\theta_{c r}$ ).

Figure 24. Comparison between bed-load transport for current only predicted by new formula (Equation 81) and measurements.

Figure 25. (a) Typical wave velocity variation, and (b) instantaneous Shields parameter variation over wave period in direction of wave propagation.

Figure 26. Calibration of coefficient $a_{w}$ using all experimental data with waves only (Nikuradse roughness calculated using Wilson (1989a) formula).

Figure 27. Comparison between bed-load transport predicted by Equation 90 and experimental data with waves (a) where $k_{s}$ is calculated using Wilson (1989a) formula, and (b) where $k_{s}=2 d_{50}$.

Figure 28. Comparison between bed-load transport predicted by Equation 90 and experimental data over full wave cycle ( $k_{s}$ calculated using Wilson (1989a) formula, $k_{s}=$ $\left.k_{\mathrm{s}, \mathrm{siw}}\right)$.

Figure 29. Definition sketches for (a) wave and current interaction, and (b) typical velocity variation over wave period in direction of wave propagation including effect of steady current.

Figure 30. Comparison between bed-load transport predicted by Equation 99 and experimental data with current $\left(U_{c}>0.02 \mathrm{~m} / \mathrm{sec}\right)(a)$ where $k_{s}$ is calculated using Wilson (1989a) formula, and (b) where $k_{s}=2 d_{50}$.....

Figure 31. Phase-lag effect on sediment transport for sinusoidal wave with superimposed current when phase lag $\phi_{s}$ is introduced for concentration at bottom: (a) instantaneous variation in velocity, concentration, and bed-load rate for $U_{c} / U_{w}=0.2$ and $\phi_{s}=-0.2 \pi$, and

(b) effect on sediment transport for varying $\phi_{s}$ and ratio $U_{c} / U_{w}$.

Figure 32. Phase-lag effects on sediment transport for second-order Stokes wave with (a) positive, or (b) negative, and adding current introducing phase lag $\phi_{s}$ for concentration at bottom and with $r_{w}=0.2$

Figure 33. Notation for colinear wave and current interaction. 70

Figure 34. Calibration of conceptual model against data. 75

Figure 35. Influence of grain size on bed load sediment transport (details of input parameters for cases $a$ and $b$ are given in Table 11).

Figure 36. Influence of wave orbital velocity on sediment transport (values of input parameters for cases a, b, c, and d are given in Table 12).

Figure 37. Influence of wave period on sediment transport (details of input parameters for cases a, b, c, and d are given in Table 13).

Figure 38. Comparison between predicted and measured sediment transport rate using

(a) original Camenen and Larson formula, (b) Dibajnia and Watanabe formula,

(c) modified Camenen and Larson formula (Equations 99 and 107), and (d) Camenen

and Larson formula with coefficient $r_{p / 2}$ (Equations 102 and 110).

Figure 39. Computation of suspended load over depth

Figure 40. Concentration profile for steady conditions.

Figure 41. Three analytical relationships for vertical sediment diffusivity (Equations 124, 125 , and 127 ) versus $z / h$ with $\sigma=\sigma_{E}=1 / 2 \sigma_{P}=1 / 6 \sigma_{B}$.

Figure 42. Comparison between "energy slope" (ES) method and "velocity profile" method (VP) to estimate the Nikuradse roughness $k_{s}$ and shear velocity $u \star_{c}$. 
Figure 43. Vertical profile of sediment diffusivity obtained from Equation 129 using measured concentration profiles (each symbol corresponds to a particular profile)...

Figure 44. Examples of comparisons between predicted concentration profiles, using fitted exponential (solid line) or power-law profiles (dashed line), and measured concentration.

Figure 45. Comparison between predicted concentration using fitted exponential profile (a), "linear" power-law profile (b), or "parabolic" power-law profile (c), and measured concentration using all data...

Figure 46. Estimation of Schmidt number (assumed equal to $\sigma_{B}$ ) as function of ratio $W_{s} / U *_{c}$ (solid line corresponds to Equation 133, dashed line to Equation 122, proposed by Van Rijn (1984b), and dashed-dotted line to Rose and Thorne (2001) formula)..

Figure 47. Estimated coefficient $\sigma_{E}$ compared to coefficients $\sigma_{P}$ and $\sigma_{B}$.

Figure 48. Estimated values of Schmidt number as function of ratio $W_{s} / U *_{c}$, together with predictive equations.

Figure 49. Vertical profiles of eddy diffusivity obtained from Equation 130 using measured concentration profiles with interaction between nonbreaking waves and current (data from wave flumes and water tunnels; each symbol corresponds to particular profile).

Figure 50. Vertical profile of eddy diffusivity obtained from Equation 130 using measured concentration profiles with interaction between nonbreaking waves and current (data from large-scale facilities and field measurements; each symbol corresponds to particular profile).

Figure 51. High sediment concentration close to bottom using data from Dohmen-Janssen (1999) (elevation $z$ was increased by $1 \mathrm{~cm}$ to allow for logarithmic representation).

Figure 52. (a) Characteristic sediment diffusion profile (Equation 142) and induced sediment concentration profile, and (b) division of induced concentration profile to different layers and application of an exponential and parabolic logarithmic profile to estimate suspended load.

Figure 53. Schematic representation of sediment concentration within moving mixing layer for rippled bed.

Figure 54. Examples of comparison between predicted concentration using fitted exponential profile (solid line) and power-law profiles (dashed and dashed-dotted line) and measured concentration for interaction between nonbreaking waves and current (data from wave flumes, water tunnels, and basins).

Figure 55. Examples of comparison between predicted concentration using fitted exponential profile (solid line) and power-law profiles (dashed and dashed-dotted line) and measured concentration for interaction between nonbreaking waves and current (data from large-scale facilities and field measurements).

Figure 56. Examples of (a) concentration profiles, and (b) corresponding sediment diffusivity profiles, using data from Steetzel (1984) and Van der Velden (1986).

Figure 57. Dimensionless sediment diffusivity $\varepsilon_{w, E} /\left(\kappa h u *_{w}\right)$ versus (a) wave period, and (b) ratio $U_{w} / W_{s}$.

Figure 58. Vertical sediment diffusivity $\varepsilon_{w, E}$ estimated from data compiled versus $\varepsilon_{w, E}$ calculated with Equation $152\left(U_{c}<0.05\right)$.

Figure 59. Estimated value of coefficient $\sigma_{w}$ using Equation 154 as function of ratio $W_{s} / u *_{w}$ with roughness ratio $k_{s} / d_{50}$ indicated.

Figure 60. Estimation of sediment diffusivity $\varepsilon_{c w}$ by adding current- and wave-related sediment diffusivity as function of parameter $W_{s} / u_{w}$ with ratio $U_{c} / U_{w}$ emphasized. 
Figure 61 . Vertical sediment diffusivity $\varepsilon_{C w, E}$ estimated from compiled data versus $\varepsilon_{C W, E}$ calculated with Equation 156 for wave and current interaction $\left(U_{c}>0.05\right)$.

Figure 62. Comparison between estimated energy dissipation from measured wave height variation and calculated energy dissipation from bore analogy using data from

Peters (2000).

Figure 63. (a) Ratio $\varepsilon_{b \text {,meas }} / \varepsilon_{b \text {,pred }}$ versus Irribaren parameter $\xi \infty$, and (b) ratio $u{ }_{*} / W_{s}(b)$ using data from Table 24 (except Peters data).

Figure 64. Vertical sediment diffusivity $\varepsilon_{V, E}$ estimated from compiled data versus $\varepsilon_{V, E}$ calculated with Equations 157 and 175 for breaking waves.

Figure 65. Comparison between observed reference concentrations assuming an exponential profile $\left(C_{R}\right)$ or power-law profile $\left(c_{a}\right)$ at reference level $z_{a}=k_{s}=2 d_{50}$.

Figure 66. Predicted reference concentration $c_{a}$ and $c_{R}$ versus Shields parameter from various formulas.

Figure 67. Predicted reference concentration $c_{R}$ using Equations 185 and 186 versus experimental reference concentration assuming an exponential profile for concentration.

Figure 68. Bottom concentration co versus modified skin Shields parameter $\theta_{r}$ using data collected by Nielsen (1986); new equation is based on Equation 190 with calibrated coefficient value from Equation 191 (curves are plotted using a mean value for $d *$ ).

Figure 69. Histograms of grain-size distribution for current data set (a) and wave data set (b). 146

Figure 70. Reference concentration $c_{R}$ estimated from compiled data (see Table 19) versus $C_{R}$ calculated with Equation 190 and 186 with roughness ratio emphasized.

Figure 71 . Estimated roughness ratio $k_{s} / d_{50}$ versus total Shields parameter with ripple height emphasized.

Figure 72. Reference concentration $c_{R}$ estimated from data compiled for waves only versus $C_{R}$ calculated with Equations 190 and 186

Figure 73. Reference concentration $C_{R}$ estimated from compiled data set with wavecurrent interaction versus $c_{R}$ calculated with Equations 192, 193, and 186 with (a) absolute mean current $\left|U_{c}\right|$ or $(b)$ roughness ratio $k_{s} / d_{50}$, emphasized.

Figure 74. Reference concentration $c_{R}$ estimated from data compiled with wave-current interaction versus $C_{R}$ calculated with Equations 192, 193, and 186

Figure 75. Reference concentration $c_{R}$ estimated from data compiled with breaking waves (a) excluding data from Peters (2000), and (b) using data set from Peters only, versus $c_{R}$ calculated with Equations 192, 193, and 186.

Figure 76. Ratio between estimated reference concentration and predicted reference concentration from Equations 192 and 186 as function of Irribaren parameter $\xi_{\infty}$ using data sets from Table 24.

Figure 77. Reference concentration $c_{R}$ estimated from data compiled with breaking waves (see Table 33) versus $C_{R}$ calculated with Equations 192, 186, and 196.

Figure 78. Comparison between observed and calculated suspended sediment load for steady current using Equation 210 with empirical values on $c_{R}$ and $\varepsilon$

Figure 79. Comparison between observed and calculated suspended sediment load for wave-current interaction using Equation 210 with experimental values on $c_{R}$ and $\varepsilon$.

Figure 80. (a) Vertical velocity profile, and (b) sediment concentration profile, (1) inside surf zone, and (2) close to breaker line (after (a) Svendsen and Hansen 1988, and

(b) Antsyferov et al. 1983). Circles correspond to experimental data. For velocity profiles

(a), theoretical logarithmic profile is included (dashed line). For suspended load profiles

(b), solid line corresponds to an exponential profile, and the dashed line corresponds to power-law profile ( $h_{t}$ denotes the depth at wave trough level). 
Figure 81. Comparison between observed suspended sediment load and calculated load using Equation 210 and predicted values for $C_{R}$ (Equation 185) and $\varepsilon$ (Equation 164) for current only

Figure 82. Comparison between measured and calculated values of wave and current interaction for (a) reference concentration $C_{R}$ (Equation 192) and sediment diffusivity $\varepsilon$ (Equation 164), and (b) for resulting suspended sediment load using Equation 210.

Figure 83. Schematic of transport processes in asymmetric wave motion over rippled bed.

Figure 84. Phase-lag effects on sediment transport for second-order Stokes wave with (a) positive or (b) negative current introducing phase lag $\phi_{p l}$ for concentration at bottom and with asymmetry of $r_{w}=0.1$.

Figure 85. Coefficient $\alpha_{p l, s}$ as function of sediment phase lag $\phi_{p l}$ with varying values on $U_{c} / U_{w}$ and an asymmetry of $r_{w}=0.20$.

Figure 86. Notation for colinear wave and current interaction.

Figure 87. Comparison between measured and calculated net sediment transport rate using Equations 210 and 218 with $\alpha_{p l, s}=0$ (legend is same as in Figure 88).

Figure 88. Dimensionless suspended sediment transport rate as function of phase-lag parameter $p_{\text {WR. }}$.

Figure 89. Comparison between measured and estimated net sediment transport rate using (a) Van der Werf and Ribberink (2004) formula; (b) Equations 210 and 218 with $r_{p l, s}$ from Equation 213; (c) Equations 210 and 218 with $\alpha_{p l, s}$ from Equation 217; or

(d) Equation 221.

Figure 90. Influence of wave orbital velocity on sediment transport (details of input parameters for graphs a, b, c, and d are given in Table 38).

Figure 91. Influence of wave period on sediment transport (details of input parameters for graphs $a$ and $b$ are given in Table 39).....

Figure 92. Influence of wave asymmetry on sediment transport (details of input parameters for graphs $a$ and $b$ are given in Table 40).

Figure 93. Definition of current and wave direction and velocity variation at bed in direction of wave propagation.

Figure 94. Velocity profiles according to Equations 244, 245, and $246\left(U_{c}=0.4 \mathrm{~m} / \mathrm{sec}\right.$, $h=0.2 \mathrm{~m}$, and $\left.\mathrm{z}_{\mathrm{o}}=0.001 \mathrm{~m}\right)$.

Figure 95. Cross-shore variations in hydrodynamic parameters and beach profile for an LSTF experimental case (Test 1 - spilling breakers) together with (a) measured longshore suspended sediment transport, and (b) calculated transport using six studied formulas..

Figure 96. Cross-shore variations in hydrodynamic parameters and beach profile for an LSTF experimental case (Test 6 - plunging breakers) together with (a) measured longshore suspended sediment transport, and (b) calculated transport using six studied formulas.

Figure 97. Cross-shore variations in hydrodynamic parameters and beach profile for Sandy Duck experiment (31 March 1997) together with (a) measured longshore suspended sediment transport, and (b) calculated transport using six studied formulas.

Figure 98. Cross-shore variations in hydrodynamic parameters and beach profile for Sandy Duck experiment (4 February 1998) together with (a) measured longshore suspended sediment transport, and (b) calculated transport using six studied formulas. 198

Figure 99. Predictive results for longshore sediment transport rate across beach profile using present formula for (a) LSTF data, and (b) Sandy Duck data.

Figure 100. Cross-shore variations in hydrodynamic parameters and beach profile for (a) Sandy Duck experimental case (12 March 1996) together with measured cross-shore suspended sediment transport, and (b) calculated transport using six studied formulas. 
Figure 101. Comparison of cross-shore suspended load across profile line with present formula and Sandy Duck data.

\section{Tables}

Table 1. Classification of different types of sands (after Falques and Swart 1998). ......................... 8

Table 2. Internal friction coefficient (after Migniot 1977)................................................................. 9

Table 3. Summary of data sets on inception of sheet flow under oscillatory flow.............................28

Table 4. Prediction of critical wave orbital velocity for inception of sheet flow within factor

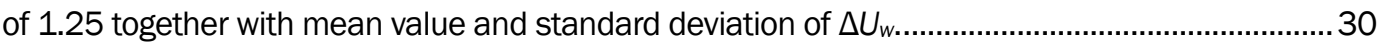

Table 5. Data base compiled to study bed-load sediment transport in steady current (plane bed cases only).

Table 6. Prediction of bed-load transport rate within factor of 2 and 5 of measured values and root-mean-square errors using current only data...................................................................... 45

Table 7. Data summary for bed-load sediment transport experiments carried out in oscillatory flow with and without current.

Table 8. Predictive capability of bed-load transport rate within factor of 2 and 5 of measured values and root-mean-square errors, data from waves only.

Table 9. Prediction of bed-load transport rate within factor of 2 and 5 of measured values and root-mean-square errors using data on waves and current combined $\left(\left|U_{c}\right|>0.02\right.$ $\mathrm{m} / \mathrm{sec})$

Table 10. Summary of data on bed-load sediment transport in full-cycle oscillatory flow................73

Table 11. Experimental conditions for studied cases on median grain size effect. .......................... 76

Table 12. Experimental conditions for studied cases on wave orbital velocity effects...................... 77

Table 13. Experimental conditions for studied cases on wave period effects (for Dibajnia experiments, maximum onshore wave orbital velocity is approximately constant).

Table 14. Prediction of bed-load transport rate within factor of 2 or 5 of measured values,

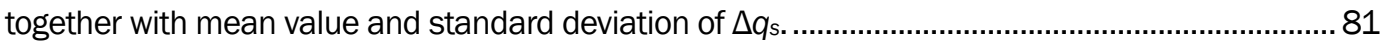

Table 15. Data summary for suspended sediment experiments under steady currents. ................92

Table 16. Percentage of predictions of sediment concentration within $+/-20$ percent of measured values obtained using an exponential law or power law (linear and parabolic profile) for $\varepsilon_{c}$ in fitting against data.

Table 17. Data summary for analysis on Schmidt number $\left(d_{s}\right.$ corresponds to median grain size in suspension).

Table 18. Prediction of Schmidt number using parabolic profile or an exponential profile for steady current. .

Table 19. Data summary for suspended sediment experiments under oscillatory flows. 104

Table 20. Percentage of predicted sediment concentrations within $+/$ - 20 percent of measured values using exponential-law or power-law (linear and parabolic profiles for $\left.\varepsilon_{w}\right)$ profiles for studied data sets.

Table 21. Input parameters for four study cases in Figure 56.

Table 22. Predictive skill of different formulas for sediment diffusivity for waves only.

Table 23. Prediction of sediment diffusivity for wave and current interaction (for different calculations of $\varepsilon_{c w, E}$, Equations 136 and 154 are used for obtaining $\varepsilon_{c, E}$ and $\varepsilon_{w, E}$, respectively). 
Table 24. Data summary for suspended sediment experiments along beach profiles employed for investigating breaking wave effects (for random waves, $H_{w, \infty}=H_{m o, \infty}$ and $T_{w}$ $=T_{p}$ ).

Table 25. Prediction of sediment diffusivity for transport under breaking waves.

Table 26. Selected relationships for reference concentration (chronological order)

Table 27. Prediction of reference concentration assuming parabolic power-law or an exponential sediment concentration profile.

Table 28. Experimental data used by Nielsen (1986)

Table 29. Prediction of bottom concentration using data from Nielsen (1986).

Table 30. Prediction of reference concentration using studied data sets encompassing waves only.

Table 31. Prediction of reference concentration using compiled data set with wave-current interaction.

Table 32. Prediction of reference concentration using compiled data set with breaking waves.

Table 33. Data summary for suspended sediment experiments under oscillatory flows (GS35 and GS45 correspond to "Grote Speurwerk" with length of 35 and 45 m, respectively).

Table 34. Prediction of suspended load transport for steady current.

Table 35. Prediction of suspended load transport for interaction between current and waves.

Table 36. Summary of data on suspended sediment transport over ripples in full-cycle oscillatory flow.

Table 37. Prediction of suspended sediment transport rate within factor of 2 or 5 of measured values, together with mean value and standard deviation on $f\left(q_{\mathrm{ss}}\right)$.

Table 38. Experiment conditions for studied cases on wave orbital velocity effects (mean value is used for ripple height $H_{r}$; for Grasmeijer (2002) experiment, mean value is also used for water depth $h$ and velocity $U_{c}$ ).

Table 39. Experiment conditions for studied cases on wave period effects (mean value is used for ripple height $H_{r}$ ).

Table 40. Experiment conditions for studied cases on wave asymmetry effects (mean value is used for ripple height $H_{r}$ ).

Table 41. Experiment conditions for studied cases on longshore sediment transport

Table 42. Predictive capability of different transport formulas for longshore suspended load transport for LSTF and Sandy Duck experiments.

Table 43. Predictive capability of different transport formulas regarding suspended load transport in cross-shore direction for Sandy Duck experiments. 203

Table 44. Predictive capability of total load sediment transport in cross-shore direction for sheet-flow experiments by Dohmen-Janssen and Hanes (2002). 


\section{Preface}

The Coastal Inlets Research Program (CIRP) is developing predictive numerical models for simulating the waves, currents, sediment transport, and morphology change at coastal inlets. Water motion at a coastal inlet can synoptically range through quasi-steady currents as in river flow, tide, wind, and seiching; oscillatory flow as under surface waves, which can create quasi-steady wave-induced currents; breaking and nonbreaking waves; and arbitrary combinations of these flows acting with different magnitudes and at different directions. Reliable prediction of morphology change requires accurate predictive formulas for sediment transport rates that will smoothly match in the aforementioned regimes of water motion and change according to the driving forces and water depth. This report describes a research effort conducted with the aim of developing unified sediment transport rate formulas for application in the coastal inlet environment. These formulas, calibrated with a wide range of available measurements compiled from the laboratory and field, have been implemented in CIRP's Coastal Modeling System.

CIRP is administered at the U.S. Army Engineer Research and Development Center (ERDC), Coastal and Hydraulics Laboratory (CHL) under the Navigation Systems Program for Headquarters, U.S. Army Corps of Engineers (HQUSACE). J ames E. Walker is HQUSACE Navigation Business Line Manager overseeing CIRP. J ames E. Clausner, CHL, is the Technical Director for the Navigation Systems Program. Dr. Nicholas C. Kraus, Senior Scientists Group (SSG), CHL, is the CIRP Program Manager.

The mission of CIRP is to conduct applied research to improve USACE capability to manage federally maintained inlets, which are present on all coasts of the United States, including the Atlantic Ocean, Gulf of Mexico, Pacific Ocean, Great Lakes, and U.S. territories. CIRP objectives are to advance knowledge and provide quantitative predictive tools to (a) make management of Federal coastal inlet navigation projects, principally the design, maintenance, and operation of channels and jetties, more effective and reduce the cost of dredging, and (b) preserve the adjacent beaches and estuary in a systems approach that treats the inlet, beaches, and estuary as sediment-sharing components. To achieve these objectives, CIRP is organized in work units conducting research and development in hydrodynamic, sediment transport and morphology change modeling; 
navigation channels and adjacent beaches; navigation channels and estuaries; inlet structures and scour; laboratory and field investigations; and technology transfer.

This report was prepared under contract with CIRP by Dr. Magnus Larson, Department of Water Resources Engineering, Lund University, Sweden, and by Dr. Benoît Camenen, presently at Cemagref Lyon, France, and formerly a post-doctoral researcher at Lund University, Sweden, and at the Disaster Prevention Research Institute, Kyoto University, J apan. J . Holley Messing, Coastal Engineering Branch, Navigation Division, CHL, typed the equations and format-edited this report. Dr. Kraus oversaw technical elements of this project during the 3 years of required research and development. Thomas W. Richardson was Director, CHL, and Dr. William D. Martin, Deputy Director, CHL, during the study and preparation of this report.

COL Richard B. J enkins was Commander and Executive Director of ERDC. Dr. J ames R. Houston was Director. 


\section{Introduction}

\section{Background}

Many sediment transport formulas have been developed through the years for application in coastal areas (for example, see Bayram et al. 2001; Camenen and Larroudé 2003). However, these formulas have typically described a specific set of physical processes, which limits their applicability in a situation where many processes act simultaneously to transport the sediment as, for example, around a coastal inlet. Also, most formulas have been validated with limited data. Thus, there is a lack of general and consistent sediment transport formulas valid under a wide range of hydrodynamic, sedimentologic, and morphologic conditions that yield reliable and robust predictions. In this report, such a formulation is presented and validated against a large set of laboratory and field data on longshore and cross-shore sediment transport.

The coastal environment at an inlet encompasses hydrodynamic forcing of many types, where waves, tides, wind, and river runoff are the most important agents for initiating water flow and associated sediment transport. Besides the oscillatory motion, waves induce mean currents in the surf zone (longshore currents, rip currents, etc.), stir and maintain sediment in suspension through the breaking process, and cause swash motion and transport on the foreshore. The wind and tide generate mean circulation patterns that move sediment, especially in combination with waves. Also, on the bay side and in the vicinity of the inlet throat, river discharge to the bay might generate currents that significantly contribute to the net transport. Figure 1 schematically illustrates some of the hydrodynamic forcing around an inlet that contributes to the mobilization and transport of sediment. 


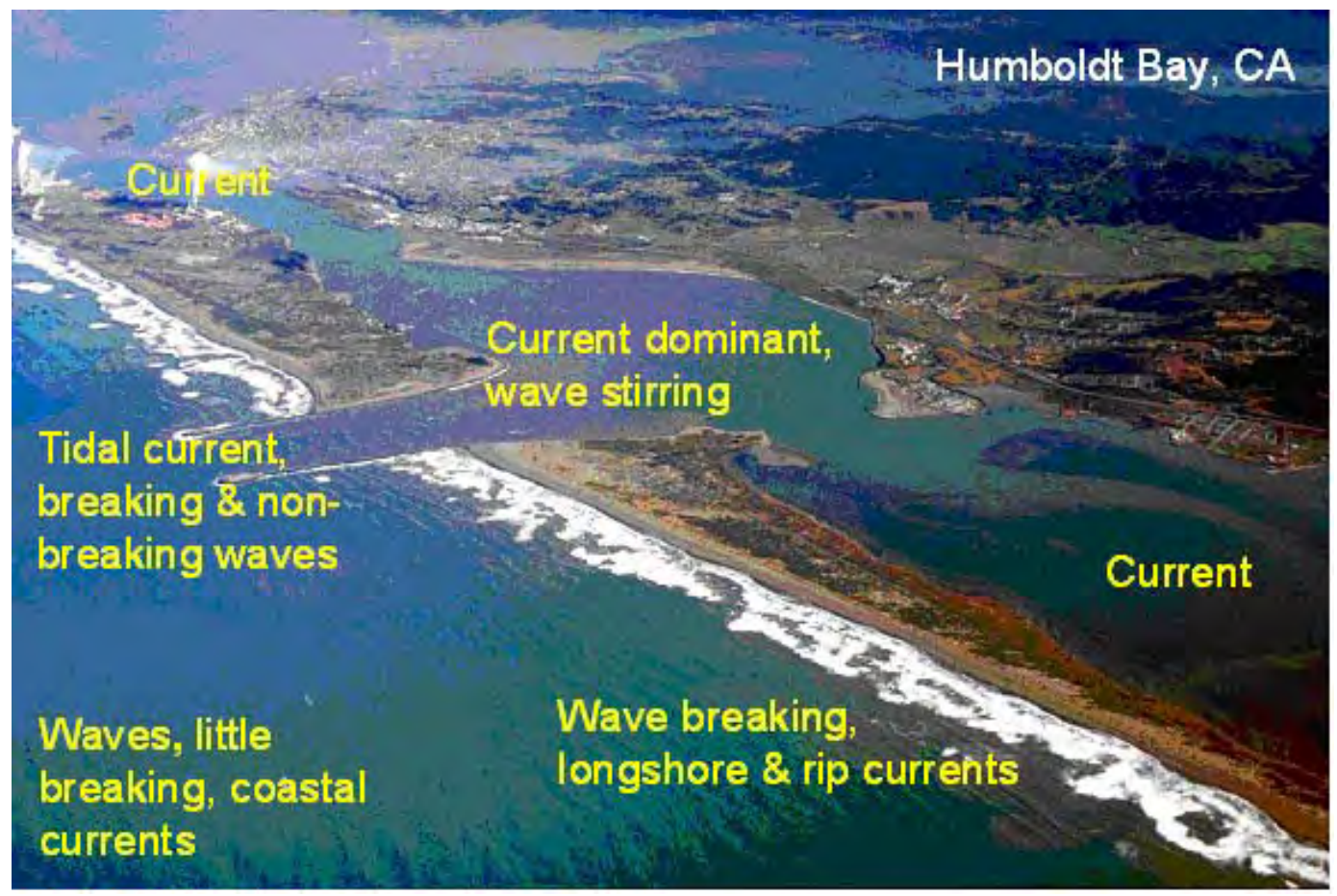

Figure 1. Hydrodynamic processes controlling sediment transport in an inlet environment (slide courtesy of N. C. Kraus).

Predicting sediment transport and morphologic evolution around a coastal inlet is necessary in support of engineering activities that ensure proper functioning of the inlet for navigation (Figure 2). Optimizing dredging operations in response to channel shoaling or minimizing local scour, which may threaten jetty integrity, are examples of such activities. Furthermore, natural bypassing of sediment through the inlet shoals and bars is required to supply material to downdrift beaches, and a reduction in this transport may cause erosion and shoreline recession. After an inlet opens, as the shoals and bars grow with little bypassing transport, downdrift erosion is common, and varying engineering measures such as beach nourishment and structures might be needed. On the updrift side of an inlet, accumulation normally occurs, especially if the inlet has been stabilized with jetties, with shoreline advance and increased infilling of the channel. 


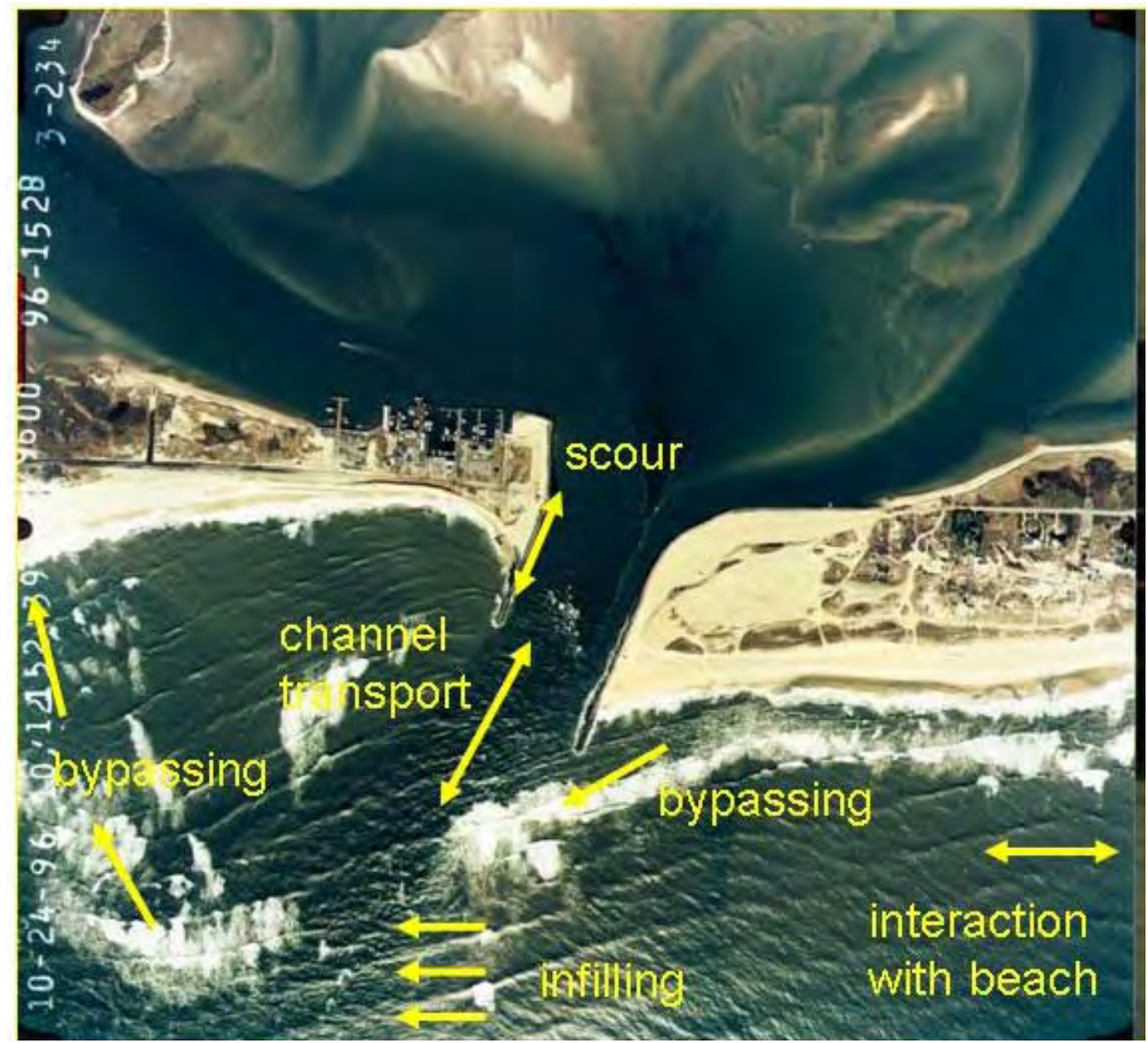

Figure 2. Natural processes around an inlet for which predictions of sediment transport and morphological evolution are of importance (slide courtesy of N. C. Kraus).

For these kinds of engineering-support studies, a gap has existed between semi-empirical approaches yielding estimates of transport rates for small scales (e.g., for a fixed water depth and with a temporal average over several wave periods) and the more empirical approach giving an assessment of mean sediment rate at larger scales (e.g., mean value for the surf zone based on formulas calibrated against long-term data). Here, the main objective is to develop reliable formulas that can predict net transport rates yielding the daily evolution (including storms), as well as the monthly and yearly evolution (seasonal and long-term forcing) in support of predictive numerical models of morphology change and channel evolution at coastal inlets for engineering design and planning. The main imposed hydrodynamic forcing for the sediment transport is the mean current (e.g., wave-induced longshore current and tidal current). 
If the cross-shore evolution of the beach is neglected, the influence of waves can be simplified as a stirring effect (Figure 3). Emphasis is given to suspended sediment and the action of breaking waves on the suspension. Moreover, to solve problems of channel infilling, the contribution of gravity should be included. With the objective of simulating long-term evolution, simplifications are introduced for many of the governing phenomena to limit computation time and reduce input requirements.

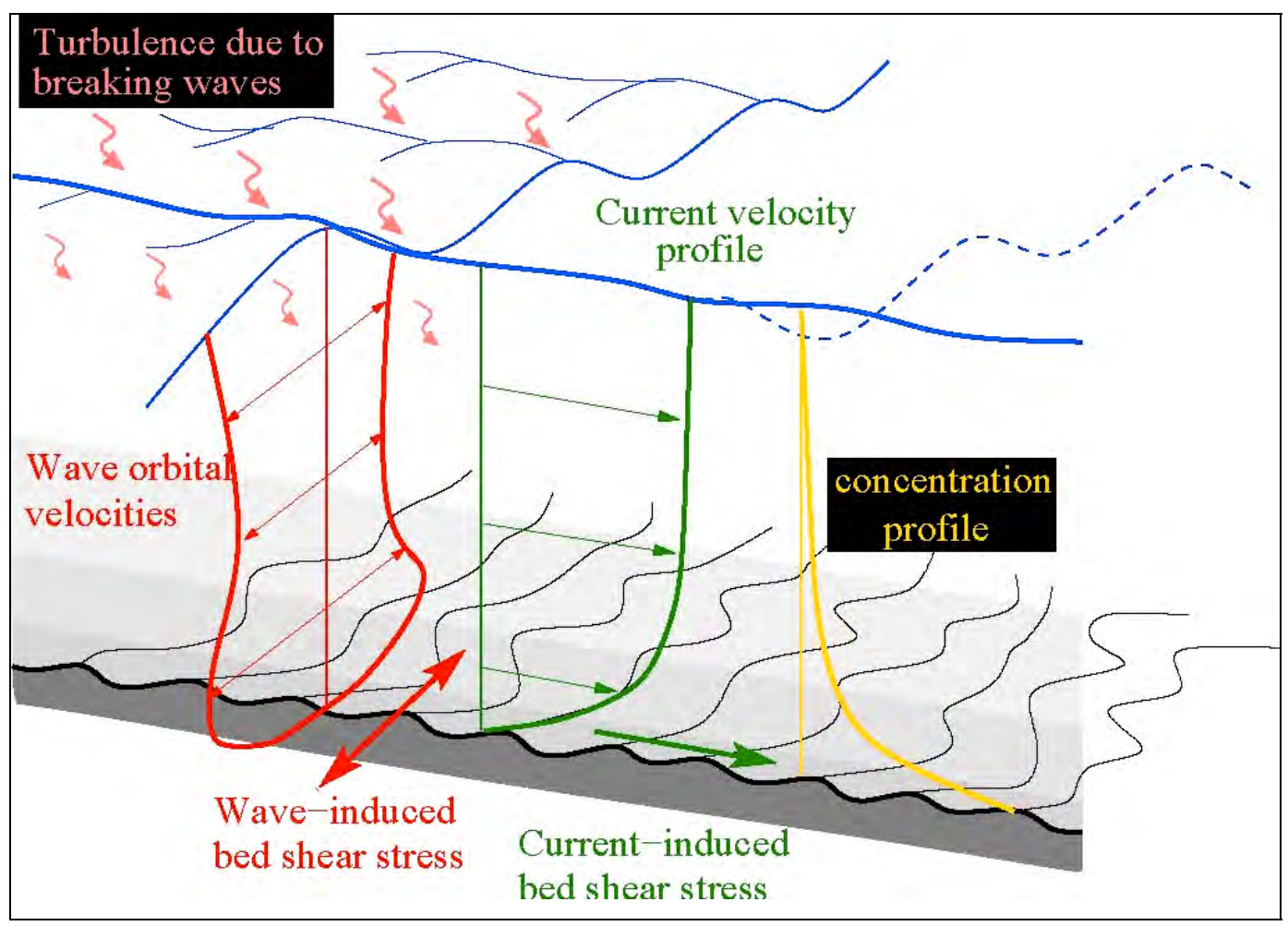

Figure 3. Hydrodynamic forcing determining conditions for longshore sediment transport (after Soulsby 1997).

Elements of the sediment transport formulation undertaken in the present study can be summarized as follows:

1. Develop sediment transport rate formulas valid at several different scales (a variety of formulas is needed).

2. Target both a general detailed model (modeling system) and predictive models for more specific purposes.

3. Build on previous work (existing formulas) for a wide variety of conditions, but identify limitations and extend applicability by enhancements.

4. Validate with both "point" and "area" data (from laboratory and field). 
5. Test models for a variety of conditions to ensure robustness, reliability, and efficiency.

6. Introduce morphologic constraints, as required.

For this kind of study, the transport modeling system employed is classical in requiring or including typically available or calculated information.

Figure 4 shows the principal components of such a system. An example is the Coastal Modeling System developed in the Coastal Inlets Research Program (Buttolph et al. 2006).

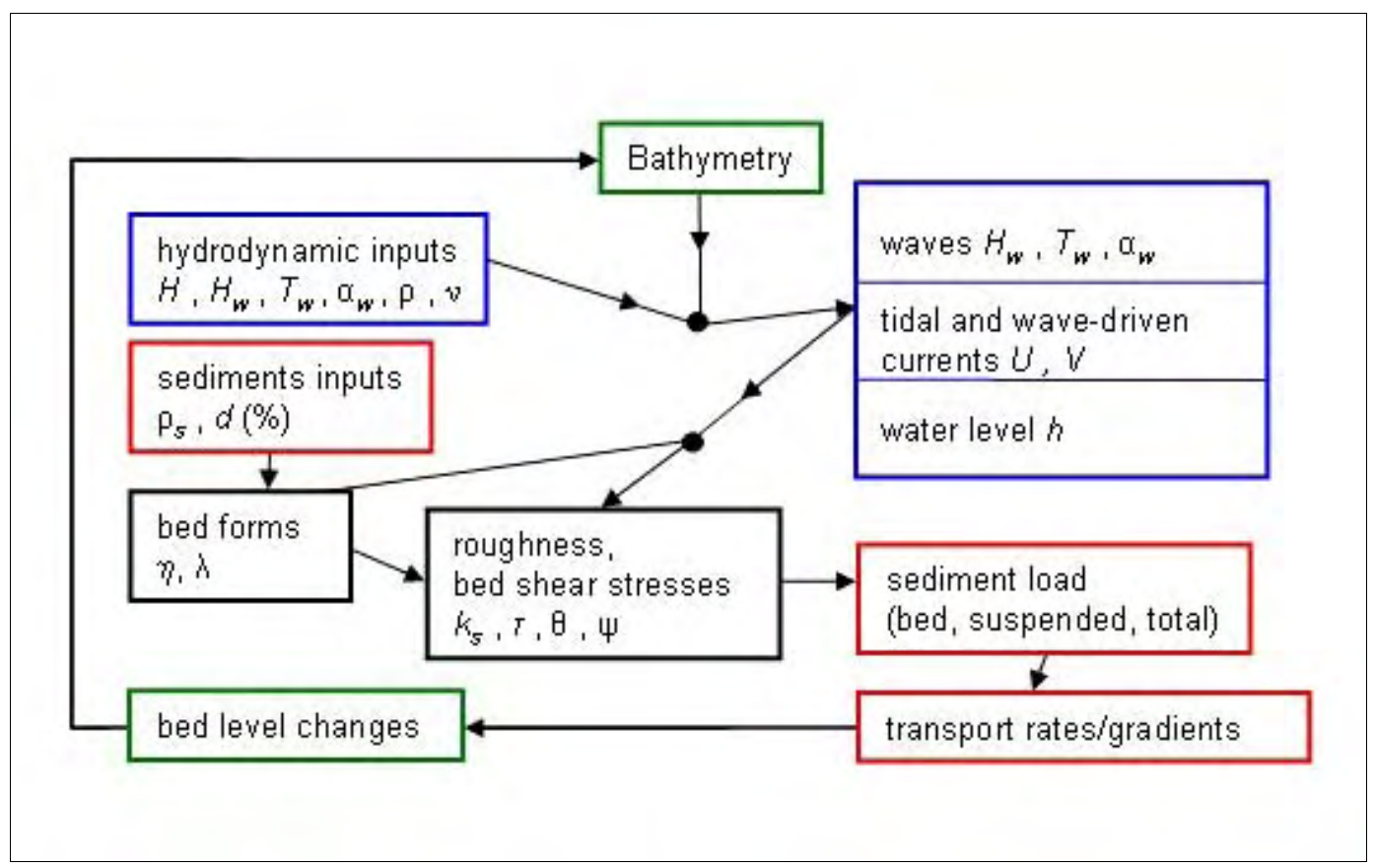

Figure 4. Sediment transport formulation.

\section{Objectives}

There is a great need for predictive sediment transport rate formulas that take into account a wide range of physical factors and conditions encountered near coastal inlets and their navigation channels. Such formulas should be compared with measurements from the laboratory and field including many different situations to ensure robust and reliable behavior. A general sediment transport formula should yield predictions of the transport rate for the following conditions:

1. Bed load and suspended load.

2. Waves and currents.

3. Breaking and nonbreaking waves. 
4. Bed slope.

5. Initiation of motion.

6. Asymmetric wave velocity.

7. Arbitrary angle between waves and current.

In this report, general formulas to compute the sediment transport rate developed that are valid under these conditions. Other phenomena or mechanisms may also be of importance, such as the phase lag between water and sediment motion or the influence of bed forms. Modifications to the general formula are derived to take these phenomena into account. The theoretical descriptions of the various mechanisms appearing for the conditions listed here are of differing sophistication, and not all of them can easily be validated with data at this time. Thus, another crucial feature of a general sediment transport formulation is that the different components can be replaced as improved descriptions become available.

The purpose of the present report is to develop a general sediment transport formulation to be applied in an inlet environment where both waves and currents control the transport rate. Emphasis on the development is to arrive at a formulation that is robust and yields reliable predictions over a wide range of input conditions. Also, all relevant physical processes should be included to obtain a formula of greatest generality. Initial development may include simpler formulations for some of these processes, but as physical understanding improves, such components may be replaced by more comprehensive approaches. Because the primary objective was to develop a robust and reliable formulation applicable under many different conditions, a large database on sediment transport measurements in the laboratory and field was assembled to test different aspects of the formulas to assure that they join smoothly through various combinations of forcing conditions.

\section{Procedure}

The strategy in the development was to derive straightforward predictive formulas to describe various governing physical processes of sediment transport and then validate these with data. At the initial stage, comparisons were made between existing formulas and data to see how well they performed and if these formulas could serve as a starting point for development of more general formulas. First, bed load under a steady current was investigated, and an extensive database was compiled for comparison 
with existing formulas. To improve the predictive capability, a slightly modified version of these transport formulas was developed that includes a different description of the criterion for incipient motion. A factor was added in the bed-load formula to describe the effect of bottom slope on the transport rate. The formula was then generalized to encompass waves at an arbitrary angle to the current, including the case of waves with an asymmetric velocity variation over a period (i.e., non-sinusoidal waves). A database on sediment transport under waves with and without a mean current was compiled, and the derived formula was compared with these data.

After studying conditions where bed-load transport was dominant, the formulation was extended to describe suspended load transport. The suspended transport rate was derived from the product of a concentration and mean velocity integrated over the water depth. An exponential function was employed to characterize the reduction in sediment concentration with distance from the bed, and a uniform velocity through the water column was assumed. Calculated sediment concentration distributions were compared with measurements, both for the case of waves and for currents. The vertical mixing coefficient, which determines the decay in concentration with distance from the bed, was expressed as a function of the energy dissipation. This dissipation includes contributions of currents and waves, where both bottom friction and breaking were taken into account for the waves. The reference concentration at the bottom was expressed in terms of the bed-load transport. 


\section{General Sediment Transport Properties}

In this chapter, selected general properties of sediment transport are discussed.

\section{Physical properties of particles}

\section{Granulometry}

Most beach sediment found is made of quartz $\left(\mathrm{SiO}_{2}\right)$. The density of the sediment is, thus, typically assumed to be $\rho_{s}=2,650 \mathrm{~kg} / \mathrm{m}^{3}$. However, shells are not negligible on some beaches, and they have a lower density ( $\rho_{s}=2,400 \mathrm{~kg} / \mathrm{m}^{3}$ for limestone).

Sediment grains are often classified according to their diameter into clays, silts, sands, granules, pebbles, cobbles, and boulders (Soulsby 1997). The characteristic diameter is usually chosen as the equivalent diameter of the sphere that has the same volume as the grain. A commonly used classification of sediments among geologist is the $\varphi_{d}$ scale defined by:

$$
\varphi_{d}=-\log _{2} d
$$

where $d$ is the sediment diameter in millimeters.

Table 1 shows the $\varphi_{d}$ scale for classification of different types of sand.

Table 1. Classification of different types of sands (after Falques and Swart 1998).

\begin{tabular}{|l|l|l|l|l|}
\hline Nature & Very Fine Sand & Fine Sand & Medium Sand & Coarse Sand \\
\hline$d(\mathrm{~mm})$ & 0.125 & 0.25 & 0.5 & 1.0 \\
\hline$\varphi_{d}$ & 3.0 & 2.0 & 1.0 & 0.0 \\
\hline
\end{tabular}

The grain-size distribution of sand may be represented by histograms with a frequency distribution or by a cumulated frequency distribution (Figure 5a and 5b, respectively). From these distributions, several parameters may be estimated: 


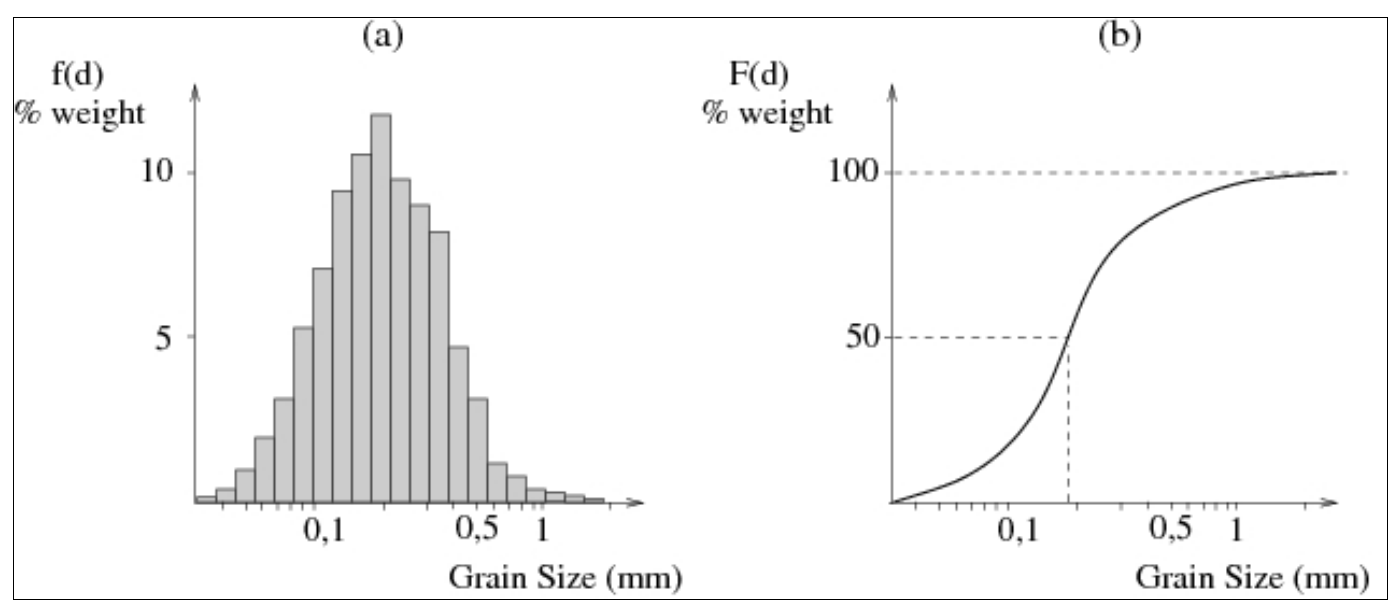

Figure 5. Example of grain-size distribution (after Fredsøe and Deigaard 1994).

- $d_{k}(0<k<100): k$ equals percent of particles by weight for which the diameter is less than $d_{k}$.

- median diameter: $d_{50}$.

- standard deviation: $\sigma=\sqrt{d_{84} / d_{16}}$.

\section{Porosity and friction angle}

The porosity is defined as follows:

$$
p=(1-c)=\left(V_{\text {tot }}-V_{\text {mat }}\right) / V_{\text {tot }}
$$

where $V_{\text {tot }}$ is the total volume, $V_{\text {mat }}$ is the volume of sediment, and $c$ the volume concentration of the sediment. For a natural mix of noncohesive sediments, $p \approx 0.4$.

The internal friction angle $\phi$ is the angle to the horizontal at which grains start to roll on a flat bed of sediment that is gradually tilted from the horizontal (Soulsby 1997). For non-cohesive sediments, the internal friction angle depends on the shape, sorting, and packing of the grains. Table 2 presents some typical values.

Table 2. Internal friction coefficient (after Migniot 1977).

\begin{tabular}{|l|l|l|l|l|}
\hline Nature & Beach Sand & Packed Sand & Gravel & Debris \\
\hline Internal friction $\phi(\mathrm{deg})$ & $28-32$ & $34-38$ & $35-40$ & $40-50$ \\
\hline
\end{tabular}




\section{Settling velocity}

A sediment particle under settling conditions in quiescent water is subject to its own weight (gravity force) and fluid resistance due to its movement (friction force). The fluid resistence is mainly characterized by the particle Reynolds number $\Re_{*}$ :

$$
\Re_{*}=\frac{W_{s} d}{\nu}
$$

where $W_{s}$ is the sediment fall speed, and $v$ is the kinematic viscosity of water (equal to $10^{-6} \mathrm{~m}^{2} / \mathrm{sec}$ at $20^{\circ} \mathrm{C}$ ). Assuming a spherical particle with a diameter $d$, a force balance leads to:

$$
\left(\rho_{s}-\rho\right) g \frac{\pi}{6} d^{3}=C_{D} \frac{\pi}{8} \rho W_{s}^{2} d^{2}
$$

where $\rho_{s}$ and $\rho$ are the density of sediment and water, respectively, and $g$ is the acceleration due to gravity. Stokes (1851) found that the drag coefficient $C_{D}$ is directly related to the particle Reynolds number $\Re_{*}$, for low values of $\Re_{*}\left(\Re_{*}<1\right)$, through the relationship:

$$
C_{D}=\frac{A}{\Re_{*}} \quad \text { if } \quad \Re_{*}<1
$$

with $A=24$ for spherical particles ( $24<A<34$ for natural sediment particles).

From Equations 4 and 5, the well-known expression for the settling velocity of spherical particles can be derived as:

$$
W_{s}=\frac{4}{3 A} \frac{(s-1) g d^{2}}{\nu}
$$

where $3 A / 4=18$ for spheres (Stokes law), and $s\left(=\rho_{s} / \rho\right)$ is the relative density. 
For large Reynolds numbers ( $\left.\Re_{*}>10^{5}\right)$, the drag coefficient is found to be constant:

$$
C_{D} \approx B \text { if } \Re_{*}>10^{5}
$$

with $B=0.39$ for spherical particles, and $1<B<1.3$ for natural sediment particles. This condition yields a settling velocity of:

$$
W_{s}=\sqrt{\frac{4}{3 B}(s-1) g d}
$$

Many semi-empirical formulas have been developed to estimate the settling velocity for weak concentrations based on these two asymptotic behaviors (see Camenen 2007). Figure 6 shows comparisons of several formulas with data from experiments with natural sand. All the studied formulas yield similar results. A simple relationship has been proposed by Soulsby (1997):

$$
W_{s}=\frac{\nu}{d}\left(\sqrt{10.36^{2}+1.049 d_{*}^{3}}-10.36\right)
$$

where $d_{*}=\left[\frac{(s-1) g d_{50}^{3}}{\nu^{2}}\right]^{1 / 3}$ is the dimensionless grain size.

If the suspended concentration of sediment increases, the settling velocity of individual particles decreases because of the return flow induced by neighboring particles (hindered settling). For example, if the mass concentration reaches $100 \mathrm{~g} / \mathrm{L}$, the fall speed becomes 50 percent less than for a single particle. The hindered fall speed may be described by the Richardson and Zaki (1954) equation based on a large set of data on sedimentation and fluidization:

$$
\frac{W_{s h}}{W_{s}}=(1-c)^{n}
$$




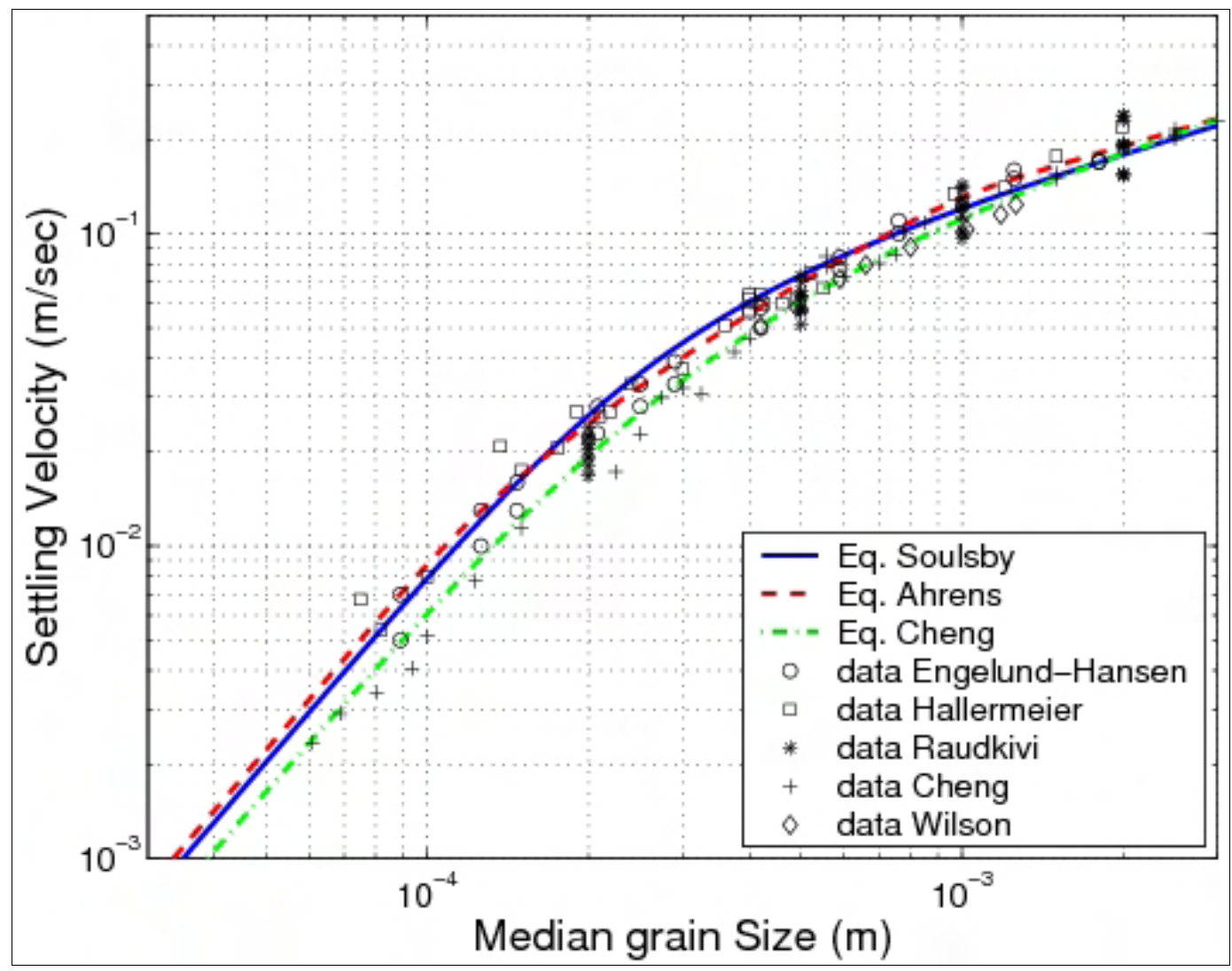

Figure 6. Settling velocity for sediment with comparison of several formulas against measurements (for details on data and equations, see Camenen 2007).

where $W_{\text {sh }}$ is the hindered settling velocity, and $2.0<n<5.0$ is an empirical coefficient. Using the limiting values of the coefficient $n$ obtained by Richardson and Zaki (1954) and assuming an infinite system, Rowe (1987) proposed an empirical fitting for $n$ as a function of the particle Reynolds number $\Re_{*}$ :

$$
n=2.35 \frac{2+0.175 \Re_{*}^{3 / 4}}{1+0.175 \Re_{*}^{3 / 4}}
$$

Camenen (2007) observed that Equation 10 is no longer valid if the concentration approaches its maximum value $c_{m} \approx 0.6$. He proposed a simple modification of the formula by Richardson and Zaki (1954).

Soulsby (1997) modified his own formula to include the effect of concentration: 


$$
W_{s}=\frac{\nu}{d}\left\{\left[10.36^{2}+1.049(1-c)^{4.7} d_{*}^{3}\right]^{0.5}-10.36\right\}
$$

Shape (angular or spherical sand), and temperature can also influence the settling velocity, yielding a deviation of as much as 50 percent from standard values (natural sand is more or less spherical).

\section{Shear stresses and friction coefficients}

\section{Bottom boundary layer flow}

With respect to sediment transport rate prediction, the most important part of the flow occurs in the bottom boundary layer. This layer is defined as that where the flow is significantly influenced by the bed (Figure 7). There are various ways to define the bottom boundary layer thickness $\delta$ in quantitative terms. In qualitative terms, Nielsen (1992) proposed the formula:

$$
\delta \approx \sqrt{\nu_{t} T}
$$

where $v_{t}$ is the eddy viscosity, and $T$ the flow period. For a steady current (e.g., tidal flow), $\delta=h$, where $h$ is the water depth, whereas for waves $\delta$ is small compared to $h$.

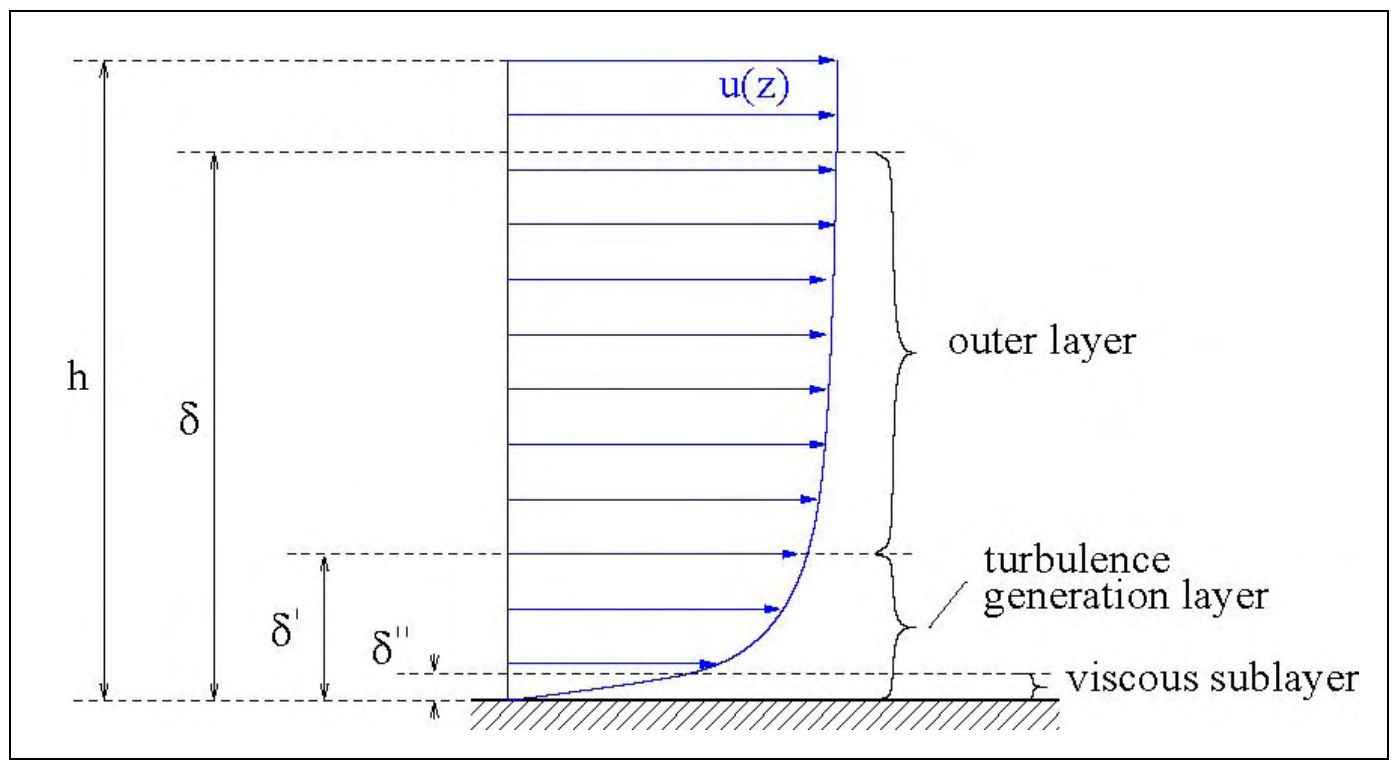

Figure 7. Turbulent boundary layer structure with mean velocity profile. 
In the viscous sublayer, turbulent fluctuations in velocity are present, but attenuated due to the presence of the bottom. Thus, the shear stress $\tau$ can be modeled approximately by the laminar boundary layer relationship:

$$
\tau=\rho \nu \frac{\partial u}{\partial z}
$$

where $u$ is the horizontal velocity. The turbulence generation layer is characterized by energetic small-scale turbulence and large fluid shear. If the bottom roughness is larger than the viscous layer, the turbulence generation layer extends to the bottom. Boussinesq (1872) introduced the eddy viscosity concept, which is defined by analogy to Equation 14 as:

$$
\tau=\rho \nu_{T} \frac{\partial u}{\partial z}
$$

The turbulent eddy viscosity can be expressed as:

$$
v_{t}=\kappa u_{*} Z
$$

where $z$ is a vertical coordinate, $\kappa(=0.4)$ the Von Karman constant, $u_{*}=\left(\tau_{b} / \rho\right)^{1 / 2}$ is called the shear velocity, and $\tau_{b}$ is the shear stress at the bed level $(z=0)$.

The outer layer is characterized by much larger eddies, which are more efficient at transporting momentum, producing a more gradually varying velocity profile compared to the turbulence generation layer.

\section{Current-related shear stress}

The bottom shear velocity for a current is defined as:

$$
u_{*_{c}}=\sqrt{\frac{\tau_{c}}{\rho}}
$$

where $\tau_{c}$ is the bottom shear stress produced by mean current. The shear velocity $u^{*}$ is a function of the velocity profile over the depth. For the classical logarithmic profile, the following relationship may be derived: 


$$
\frac{u}{u_{* c}}=\frac{1}{\kappa} \ln \frac{z}{z_{0}}
$$

where $z_{0}$ is the value of $z$ at which $u=0$.

By averaging Equation 18 over depth, the shear velocity can also be linked to the mean velocity over the depth $\left(\bar{u}=U_{c}\right)$ via the non-dimensional current drag or friction coefficients $C_{D}$ or $f_{c}$, respectively:

$$
f_{c}=2 C_{D}=2\left(\frac{u_{*}}{U_{c}}\right)^{2}
$$

The bottom shear stress can be expressed as follows:

$$
\tau_{c}=\frac{1}{2} \rho f_{c} U_{c}^{2}=\rho C_{D} U_{c}^{2}
$$

Assuming a logarithmic velocity profile (hydraulically rough flow regime), the relationship for the current friction coefficient becomes:

$$
C_{D}=\left\{\frac{\kappa}{\left[1+\ln \left(z_{0} / h\right)\right]}\right\}^{2}
$$

where $z_{0}=k_{s} / 30$ is the bed roughness length, and $k_{s, g} \approx 2 d_{50}$ holds for a plane bed, in which $k_{s, g}$ is the Nikuradse bed roughness due to skin friction, and $d_{50}$ is the median grain size.

\section{Wave-related shear stress}

In the same way, the maximum bed shear stress produced by waves is related to the bottom wave orbital velocity via a friction coefficient (J onsson 1966):

$$
\tau_{w}=\frac{1}{2} \rho f_{w} U_{w}^{2}
$$

where $U_{w}$ is the bottom wave orbital velocity, and $f_{w}$ is the wave friction coefficient. This friction coefficient is a function of the flow regime (hydraulically rough or smooth), thus a function of a wave-related Reynolds number $\mathfrak{R}_{w}$ and the relative roughness $r$ : 


$$
\Re_{w}=\frac{U_{w} A_{w}}{\nu} ; r=\frac{A_{w}}{k_{s}}
$$

where $A_{w}=U_{w} T_{w} / 2 \pi$ is the bottom wave excursion, $T_{w}$ the wave period, and $k_{s}$ the Nikuradse roughness, as previously discussed.

Because the nearshore zone is an energetic environment, only rough turbulent flows will typically occur. Several formulas have been proposed for the wave friction coefficient:

Kajiura (1968), for $r \leq 30$ :

$$
f_{w}=0.35 r^{-2 / 3}
$$

Swart (1974):

$$
\begin{aligned}
& \text { if } \quad r \leq 1.57 f_{w}=0.3 \\
& \text { if } \quad r>1.57 \quad f_{w}=0.00251 \exp \left(5.21 r^{-0.19}\right)
\end{aligned}
$$

Nielsen (1992), for all values of $r$ :

$$
f_{w}=\exp \left(5.5 r^{-0.2}-6.3\right)
$$

Soulsby (1997), for all values of $r$ :

$$
f_{w}=0.237 r^{-0.52}
$$

Figure 8 compares these equations. The result remains similar for the four studied formulas. However, some divergences appear for very low $(r<1)$ and very large $\left(r>10^{3}\right)$ relative roughnesses. 


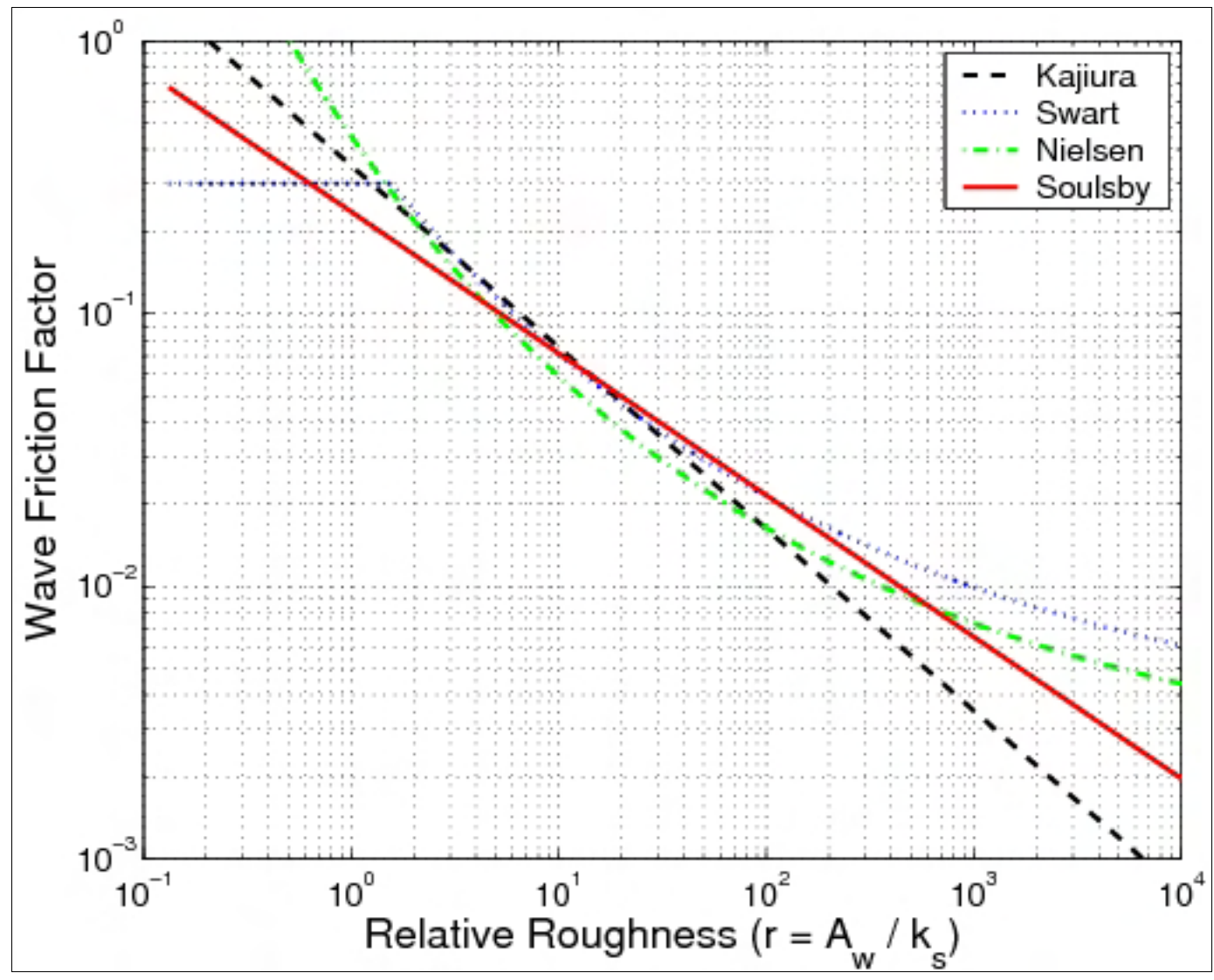

Figure 8. Wave friction coefficients for plane bed predicted by different formulas.

\section{Combined wave and current shear stress}

In most coastal processes studies, the sediment dynamics are simultaneously influenced by both waves and currents. However, the wave and current interaction is not linear, the wave phase and wavelength are modified, and current refraction of waves appears. A difficulty is to estimate nonlinear effects. Figure 9, refers to the situation $\tau_{c w, m} \neq \tau_{c}$ and $\varphi \neq \varphi_{\text {res }}$, with $\varphi$ being the angle between the mean current direction and the wave incidence direction, and $\varphi_{\text {res }}$ the angle between the resultant maximum shear stress and the mean current direction. 


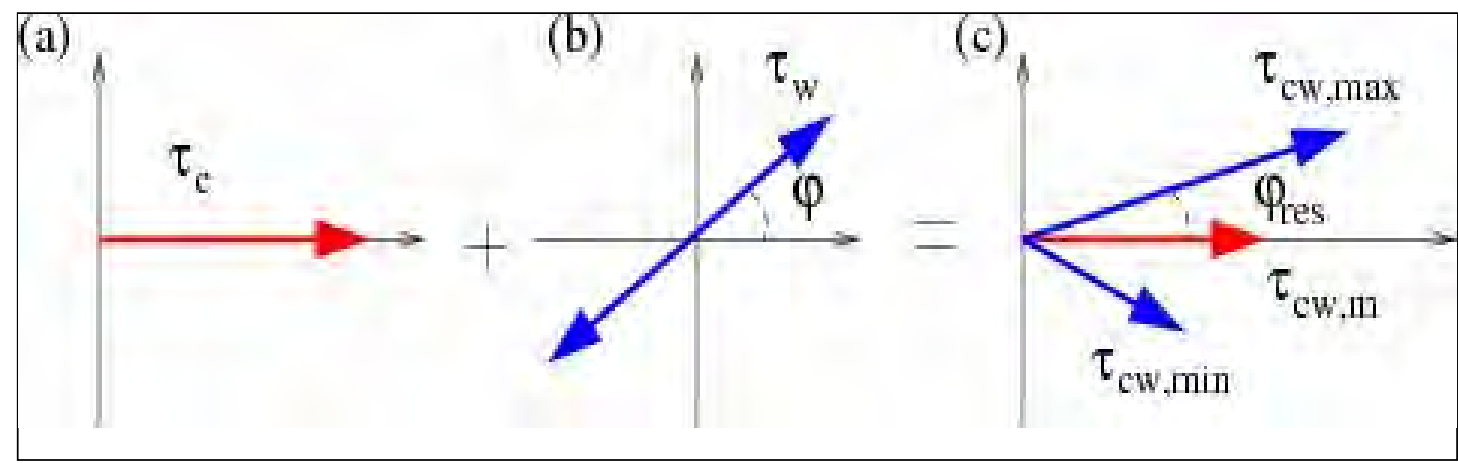

Figure 9. Schematic diagram for non-linear interaction between wave and current bed shear stress (after Soulsby et al. 1993).

Many formulas are available to compute the mean shear stress $\tau_{c w, m}$ and the maximum shear stress $\tau_{c w, \max }$ due to the wave and current interaction. Such formulas include analytical models (Grant and Madsen 1979; Fredsøe 1984) and numerical models (Davies et al. 1988; Huynh-Thanh and Temperville 1991). Soulsby (1997) proposed a simple empirical method to compute these shear stresses based on results from previous studies. Van Rijn (1984a, 1993) proposed an empirical formulation to compute mean shear stress using a simple correction term for current shear stress:

$$
\tau_{c w, m}=\alpha_{c w} \tau_{c}+\tau_{w}
$$

with $\alpha_{c w}=\alpha_{c w}\left(\delta_{w}, k_{a}, k_{s c}\right)$, where $\delta_{w}$ is the wave-boundary layer thickness, and $k_{a}$ and $k_{s c}$ are the apparent bed roughness and total bed roughness, respectively, due to the current.

For the general case of combined wave and current flow, the maximum combined bottom shear stress may be obtained from Soulsby (1997):

$$
\begin{aligned}
\tau_{c w, \max } & =\left|\vec{\tau}_{c}+\vec{\tau}_{w}\right| \\
& =\sqrt{\tau_{c}^{2}+\tau_{w}^{2}+2 \tau_{c} \tau_{w}|\cos \varphi|}
\end{aligned}
$$

\section{Bed form effects and roughness computation}

Bed forms modify the flow and sediment transport, especially sediment suspension, because the induced vortices and the bed slopes generate significant vertical velocities. These velocities carry many sand grains into suspension and change the equilibrium concentration profile of the sand. 
Figure 10 presents typical bed forms for a steady current and a simple wave orbital velocity.

(a)

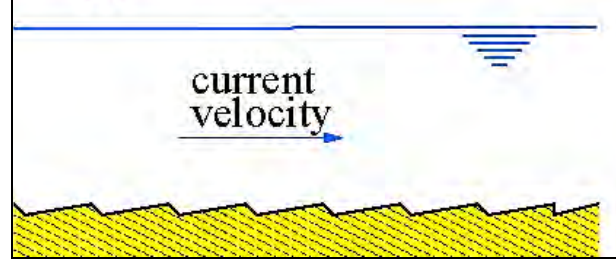

(b)

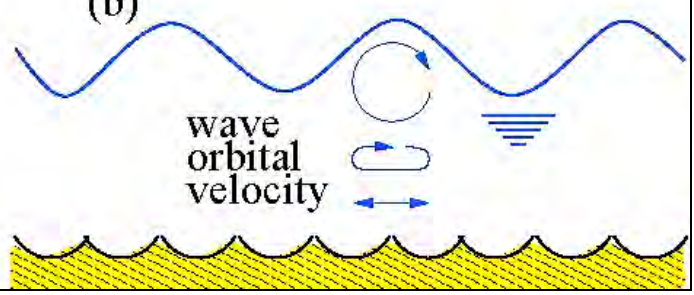

Figure 10. Schematic of ripples due to current (a) and waves (b).

\section{Current ripples, dunes, and wave ripples}

Bed forms generated by a current are asymmetric and travel downstream. In a weaker transport regime (Froude number $\mathrm{Fr}<1$ ), and erosion occurs on the stoss side and deposition on the lee side. The slope of the lee side is equal to the critical stability slope for sediment and grains move by avalanching on that side. Numerous empirical formulas exist to estimate height and wavelength of various bed forms (Yalin 1977; Van Rijn 1984c; Raudkivi 1998). A comparison is presented in Figure 11. Because significant scatter is observed in the data and the predictions of the empirical formulas, only order-of-magnitude estimates are attempted in this report.

Ripple length and height ( $L_{r c}$ and $H_{r c}$, respectively) generated by a current are mainly a function of the grain size:

$$
\begin{gathered}
L_{r c} \approx 1000 d_{50} \\
H_{r c} \approx \frac{L_{r c}}{7}
\end{gathered}
$$

Dune wavelength and height ( $L_{d}$ and $H_{d}$, respectively) are mainly a function of water depth:

$$
\begin{gathered}
L_{d} \approx 7 h \\
H_{d} \approx 0.07 L_{d}
\end{gathered}
$$




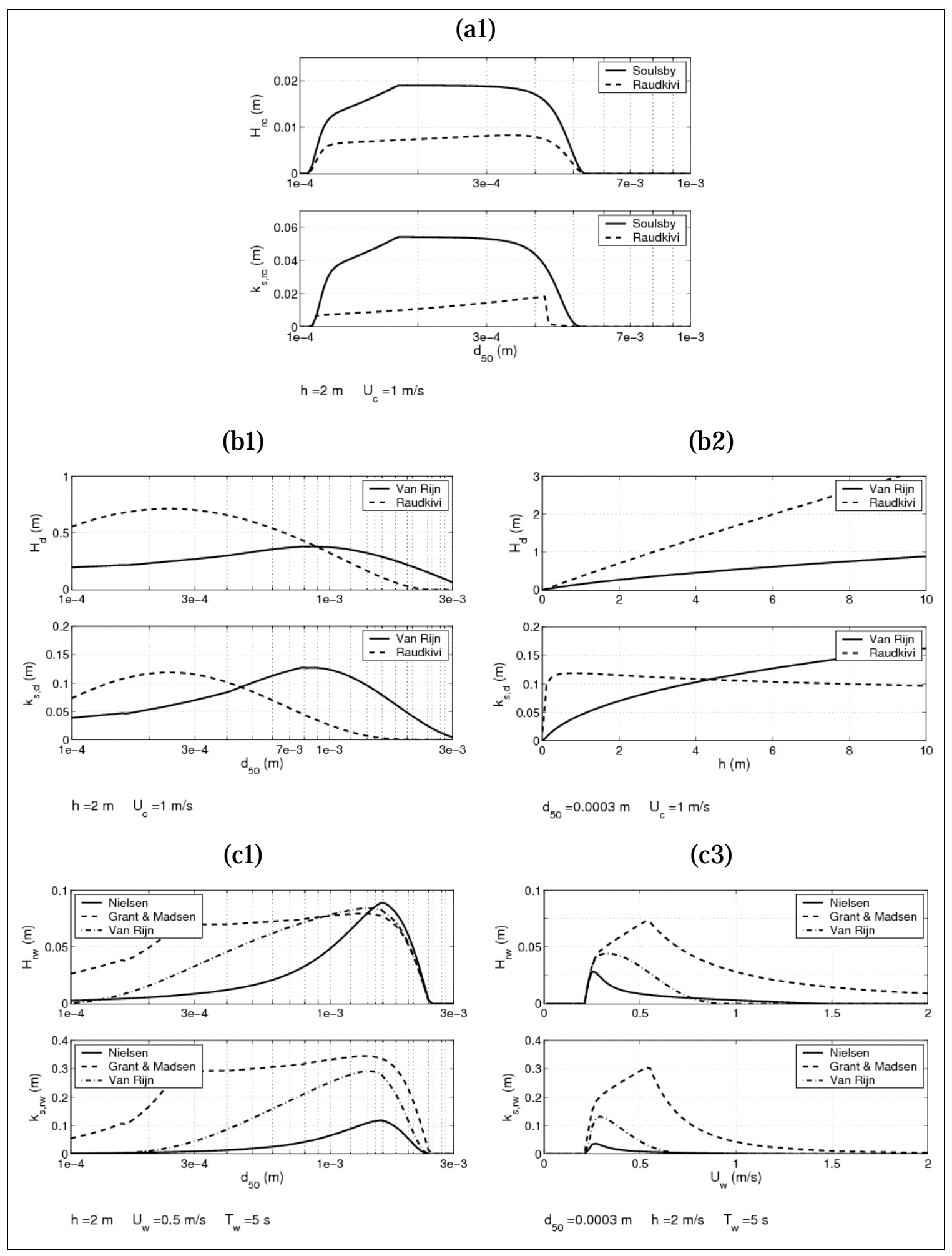

Figure 11. Influence of grain size diameter (1), water depth (2), and wave orbital velocity (3); on bed-form predictions for current ripples (a), dunes (b), and wave ripples (c); and on roughness prediction according to different formulas. 
Van Rijn (1993) proposed a relationship for dune height that is also a function of grain size, valid for the conditions that $\theta_{c r}<\theta_{c}<26 \theta_{c r}$ ):

$$
L_{d}=0.11 h\left(\frac{d}{h}\right)^{0.3}\left\{1-\exp \left[-0.5\left(\frac{\theta_{c}}{\theta_{c r}}-1\right)\right]\right\}\left[25-\left(\frac{\theta_{c}}{\theta_{c r}}-1\right)\right]
$$

where $\theta_{c}$ is the current-related Shields parameter (see Equation 48), and $\theta_{c r}$ is the threshold Shields parameter for the inception of motion.

Contrary to current-related bed forms, wave ripples are symmetric with sharper crests due to the to-and-fro movement of the wave orbital velocity. Many empirical formulas are also available to describe the properties of wave ripples (Grant and Madsen 1982; Van Rijn 1984c; Nielsen 1992) based on experimental studies yielding varying results (Figure 11). Wave ripple characteristics can be summarized through the following relationships:

$$
\begin{aligned}
& L_{r w} \approx 1-2 A_{w} \quad \text { and } \\
& 0.1<H_{r w} / L_{r w}<0.25
\end{aligned}
$$

where $A_{w}$ is the wave excursion over the bottom. The value of 0.1 corresponds to vortex formation and 0.25 to slope stability. Van Rijn (1984c, 1989) proposed a simple relationship for irregular waves where ripple properties are a function of the mobility parameter $\Psi$ :

$$
\begin{aligned}
& \Psi=\frac{U_{w}^{2}}{(s-1) g d} \\
& \frac{H_{r w}}{A_{w}}=0.22 \quad \frac{L_{r w}}{A_{w}}=1.25 \quad \text { for } \Psi_{w} \leq 10 \\
& \frac{H_{r w}}{A_{w}}=2.810^{-13}\left(250-\Psi_{w}\right)^{5} \frac{L_{r w}}{A_{w}}=1.410^{-6}\left(250-\Psi_{w}\right)^{2.5} \text { for } 10<\Psi_{w} \leq 250 \\
& \begin{array}{lll}
H_{r w}=0 & L_{r w}=0 & \text { for } \Psi>250
\end{array}
\end{aligned}
$$

Figure 11 present various results for bed-form predictions. The uncertainties involved in these kinds of computations must be noted. Indeed, for each of the displayed case studies, a factor 2 variation can be observed regarding bed-form prediction with a sensitivity that varies with water depth and grain size. 


\section{Computation of various roughnesses}

For ripples generated by a current or wave, the Nikuradse roughness can be computed in the same way:

$$
k_{s, r}=A_{r} \frac{H_{r}^{2}}{L_{r}}
$$

where $A_{r}$ varies from 10 to 30 depending on author, and the subscript $r$ denotes ripple. For mega-ripples or dunes, Van Rijn's (1984c) method can be used:

$$
k_{s, d}=1.1 H_{d}\left[1-\exp \left(-25 \frac{H_{d}}{L_{d}}\right)\right]
$$

Another form of roughness appears for sheet flow, where the "sediment fluid layer" significantly influences the boundary layer and, thus, the roughness. Several empirical formulas exist to estimate the Nikuradse roughness due to sediment transport (see Dohmen-J anssen 1999). However, significant scatter can be observed according to the author, as well as specifically through the published data (Figure 12). The most accurate relationship appears to be the equation developed by Wilson (1966, 1989a, b) based on an experimental study in a pressurized closed conduit:

$$
k_{s, s f}=5 \theta d
$$

where $\theta$ is the Shields parameter (Equation 48). It should be noted that this expression leads to an iterative computation. However, as shown in Figure 12 (Camenen et al. 2006), the Nikuradse roughness in the sheet flow regime appears to be a function also of a dimensionless settling velocity $\left(W^{*}\right)$ and/ or the suspension parameter $\left(\varpi_{s}\right)$, which are defined by, respectively:

$$
\begin{gathered}
W_{*}=\frac{W_{s}}{\sqrt{(s-1) g d_{50}}} \\
\varpi_{s}=\frac{W_{s}}{u_{*}}
\end{gathered}
$$




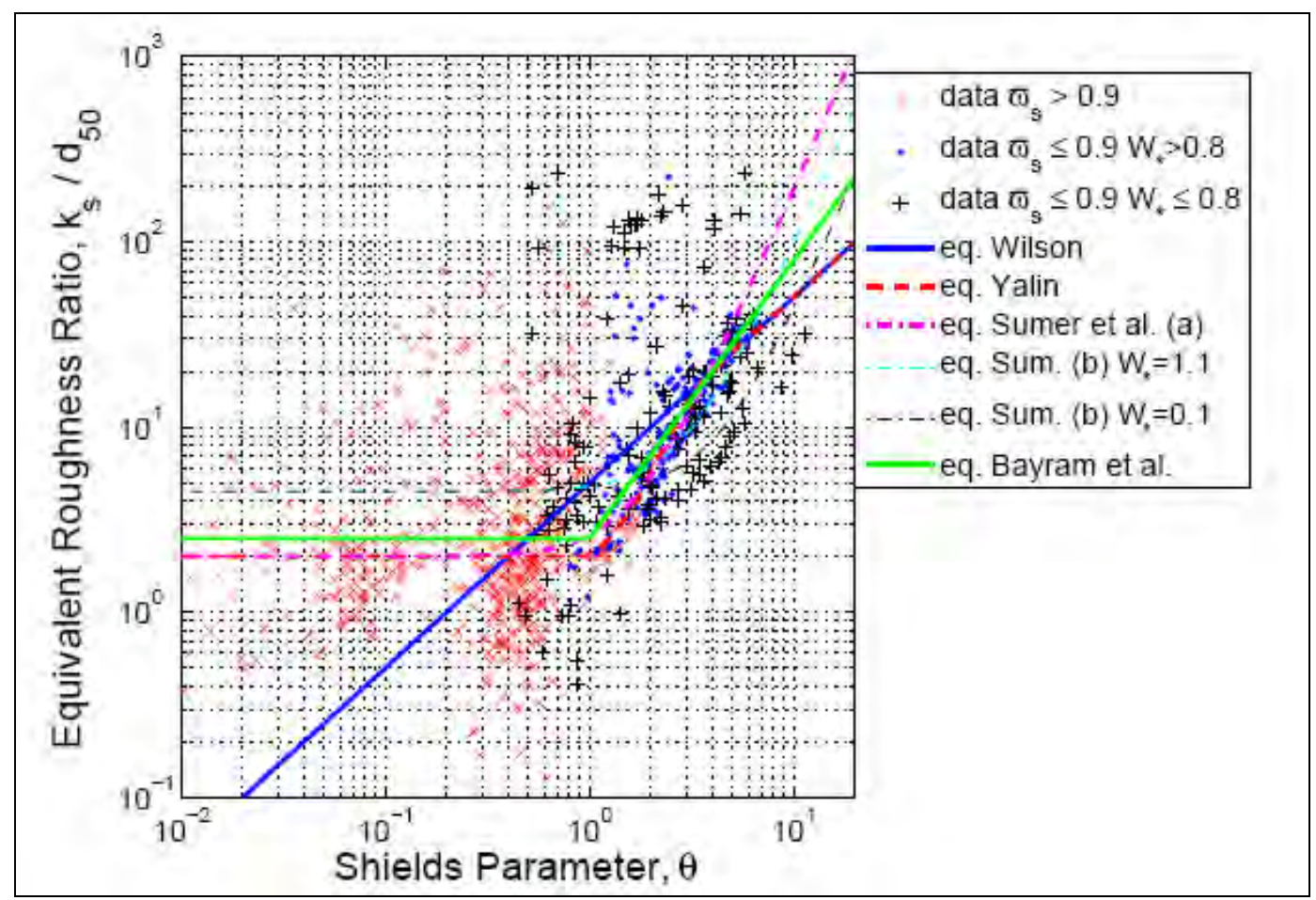

Figure 12. Equivalent roughness ratio $k_{s} / d_{50}$ versus total Shields parameter $\theta$ for compiled data set together with predictions by studied formulas (for details on data and equations see Camenen et al. 2006).

\section{Calculation of the total roughness}

A method to compute the total shear stress from the total roughness can be described by the following steps (see Soulsby 1997):

1. Current bed forms.

Calculation of the total Nikuradse roughness due to the current alone:

$$
k_{s, c}=k_{s, g c}+k_{s, r c}+k_{s, s f c}
$$

Calculation of the total friction coefficient $f_{c t}$ (see Equations 19 and 21 with $\left.z_{0}=k_{s, \mathrm{c}} / 30\right)$ :

$$
\tau_{c t}=\frac{1}{2} \rho f_{c t} U_{c}^{2}
$$

2. Wave bed forms.

Calculation of the total Nikuradse roughness due to waves only: 


$$
k_{s, w}=k_{s, g w}+k_{s, r w}+k_{s, s f w}
$$

Calculation of the total friction coefficient $f_{w t}$ (Equation 26 with $\left.z_{0}=k_{s, w} / 30\right)$ :

Calculation of the total shear stress due to waves only:

$$
\tau_{w t}=\frac{1}{2} \rho f_{w t} U_{w}^{2}
$$

3. Wave and current interaction. Calculation of the total shear stress $\tau_{c w t}=f\left(\tau_{c t}, \tau_{w t}\right)$ according to the Soulsby method (see Soulsby 1997).

\section{Shields parameter and sediment transport}

\section{Threshold of motion and critical Shields parameter}

Threshold of motion occurs if the bed shear forces acting to move the grain (proportional to $\tau_{c} d^{2}$ ) become greater than the friction forces counteracting them (assumed proportional to the submersed weight of the grain (proportional to $\left.C_{f}\left(\rho_{s}-\rho\right) g \pi d^{3} / 6\right)$. The threshold appears if the Shields parameter $\theta$ reaches a critical value:

$$
\theta=\frac{\tau}{\left(\rho_{s}-\rho\right) g d_{50}}>\theta_{c r}
$$

where $\theta$ is the Shields parameter due to current or waves, and $\tau$ the associated shear stress.

Shields (1936) noted a relationship between the critical or threshold Shields parameter and the mean grain size. Figure 13 shows results obtained from several data sets. A simple formula proposed by Soulsby and Whitehouse (1997) is given by:

$$
\theta_{c r}=\frac{0.30}{1+1.2 d_{*}}+0.055\left[1-\exp \left(-0.02 d_{*}\right)\right]
$$




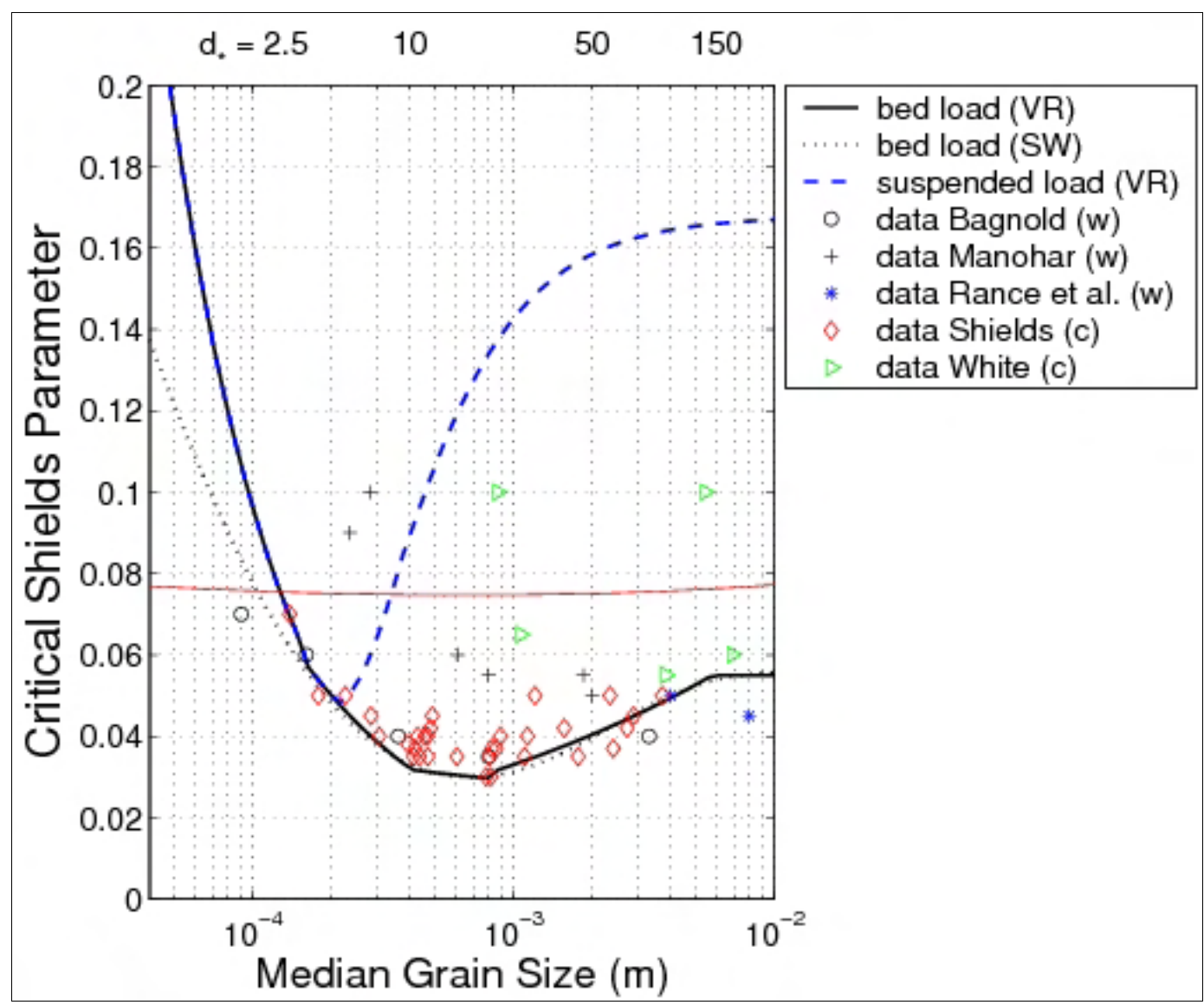

Figure 13. Critical Shields parameter plotted against dimensionless grain size (equations for bed-load limit are from Van Rijn (1993) and Soulsby and Whitehouse (1997); equation for suspended load limit is from Van Rijn (1993)), compared to data with current (c) or waves (w) (for details on data see Hanson and Camenen 2007).

Suspended load appears for slightly greater shear stresses $\left(\theta=\theta_{c r, s} \approx 0.1\right.$, Figure 13). This stage corresponds mainly to the ripples being present that induce suspended sediment. Sheet flow conditions are observed for yet greater shear stresses, above approximately $\theta=\theta_{c r, s f} \approx 0.8$.

\section{Modes of sediment transport}

Sediment transport has been categorized into three modes (Figure 14):

- Bed load: sand grains roll, slip, or jump (saltate) over the bed.

- Suspended load: sand grains are lifted over the bed and transported by the current.

Sheet flow: sand grains under imposed stresses move in large quantities as a sheet layer (a few grain diameters thick). 


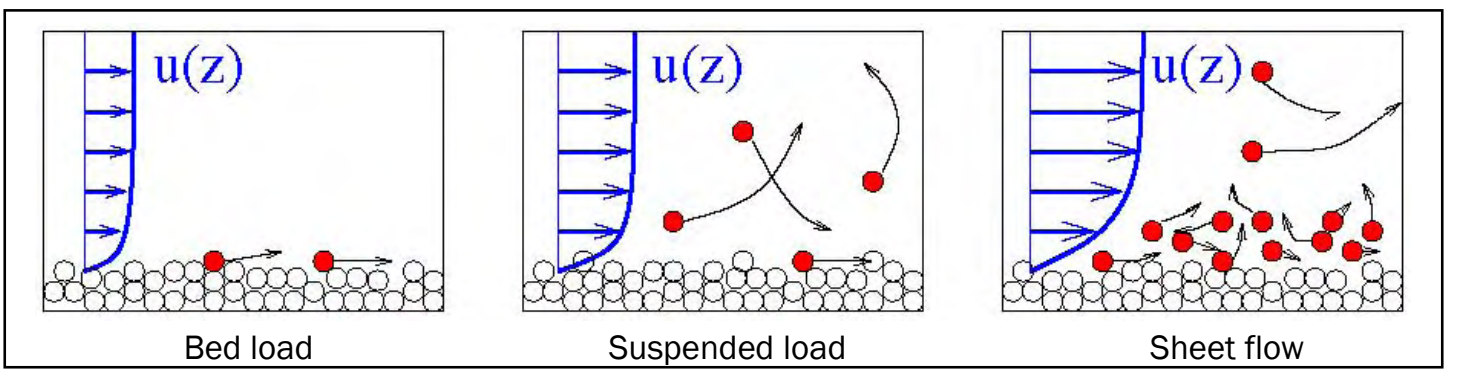

Figure 14. Modes of sediment transport (after Fredsøe and Deigaard 1994).

Considering the behavior of bed forms, for a specific sediment, the magnitude of the shear stress strongly depends on the current and/ or wave orbital velocity. Figure 15 presents a schematic view of these dependencies.

(a)

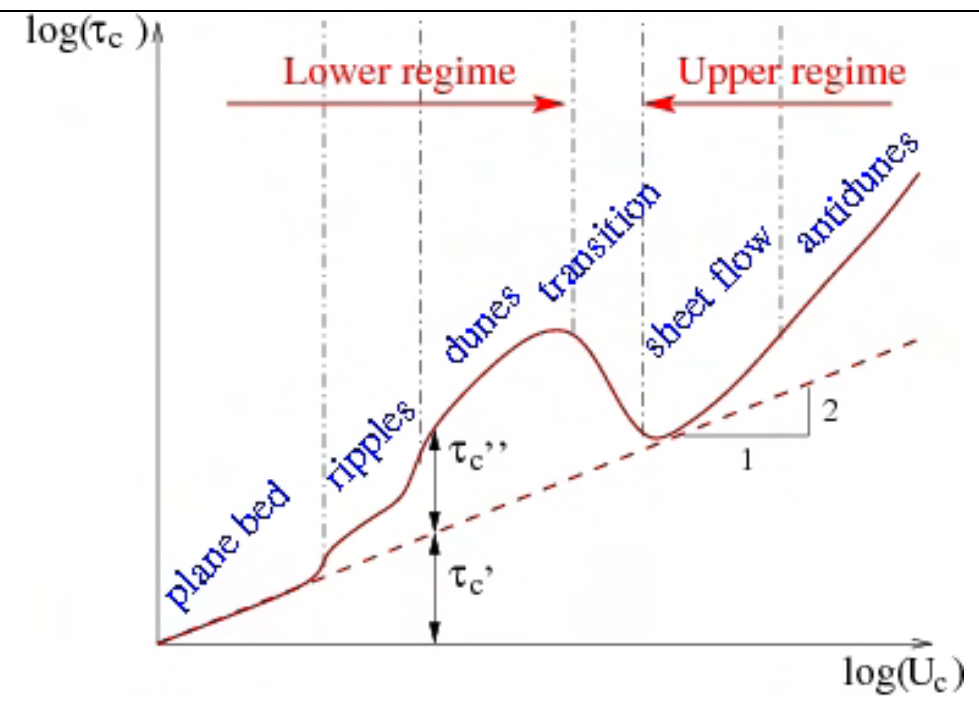

(b)

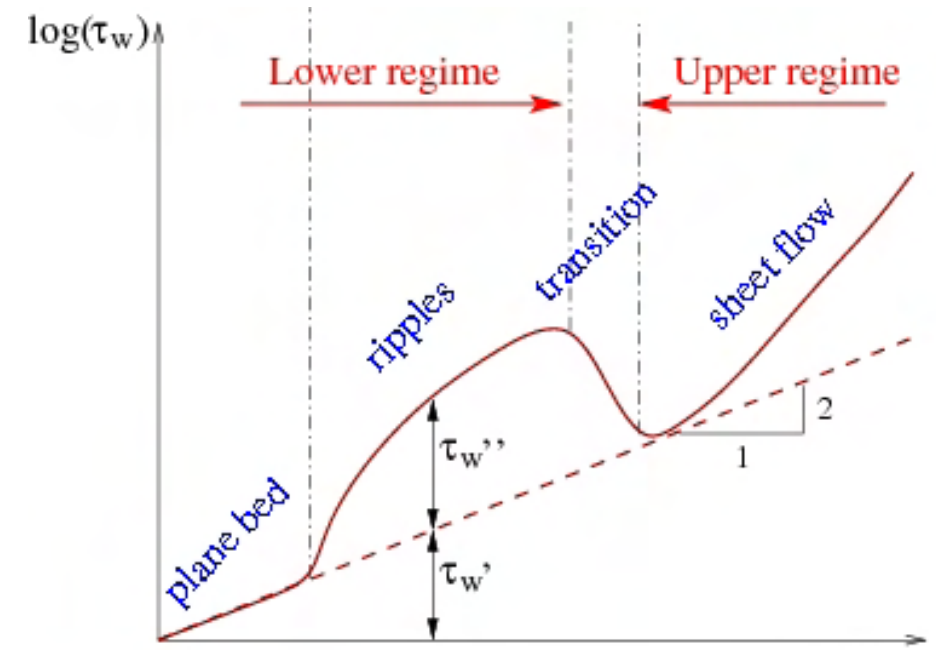

$\log \left(\overrightarrow{\mathrm{U}}_{\mathrm{W}}\right)$

Figure 15. Schematic representation of different bed forms and sediment transport regimes for (a) increasing current, or (b) wave orbital velocity. 


\section{Inception of sheet flow}

The inception of sheet flow occurs in a situation where the wave ripples are disappearing, simultaneously as the energy is increasing (increasing wave orbital velocity and/ or mean current). Typically, engineers assume that the sheet flow regime occurs when the Shields parameter reaches a critical value of $\theta_{c r, s f} \approx 0$.8. However, the Shields parameter seems to be an ineffective measure to distinguish between suspended load and sheet flow transport. Bowen (1980) and Shibayama and Horikawa (1982) proposed using the ratio $U_{c} / W_{s}$ to distinguish bed load, suspended load, and sheet flow mode in an energetics model of sediment transport. This is illustrated in Figure 16.

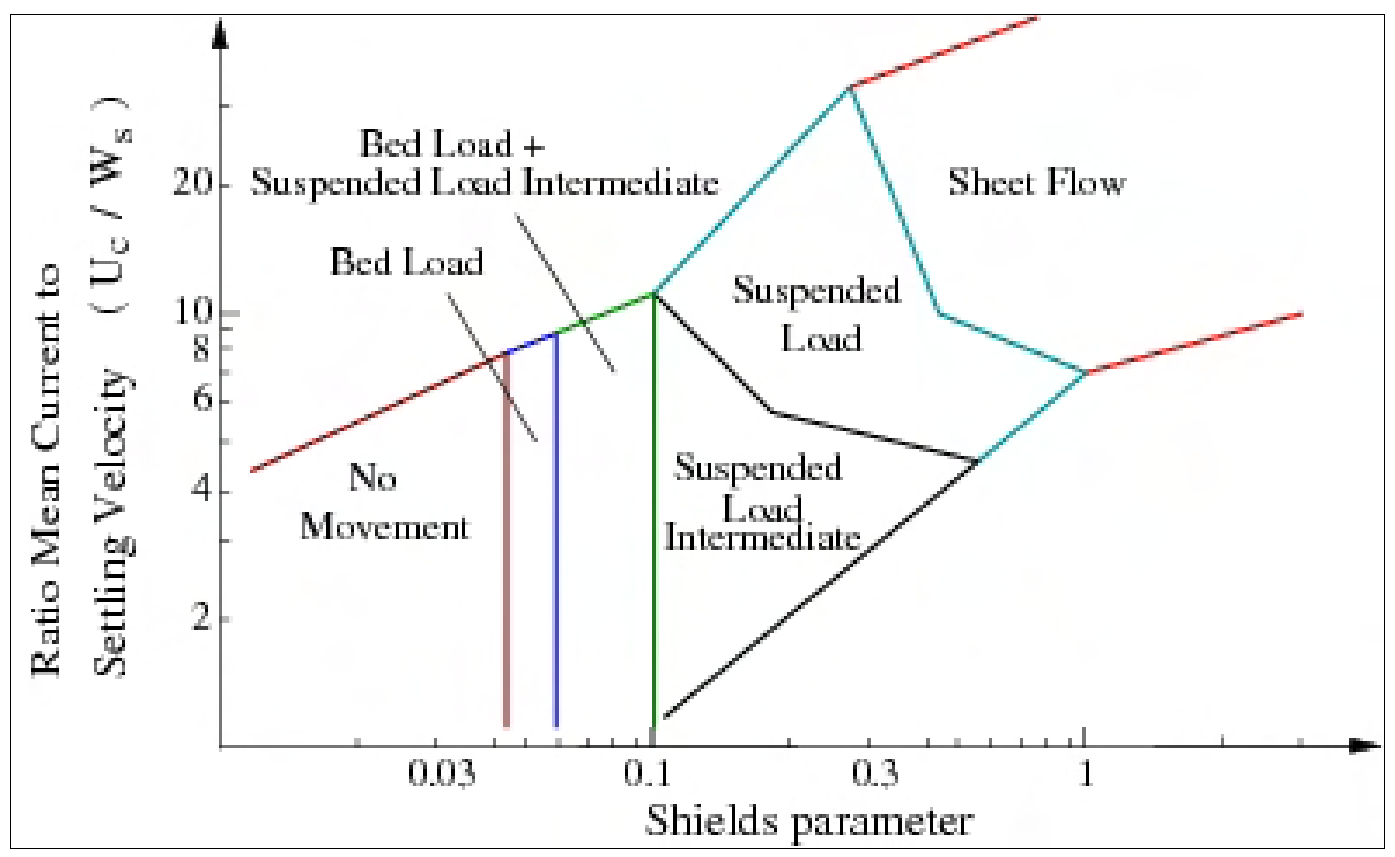

Figure 16. Classification of different types of sediment transport with respect to Shields parameter $\theta$ and ratio $U_{c} / W_{s}$ (after Shibayama and Horikawa 1982).

Previous studies. In an extensive investigation, Manohar (1955) was the first to study the initiation of sheet flow by means of an oscillatory tray (OT). Chan et al. (1972) used a horizontal tube (HT) to investigate the behavior of a bed of particles under oscillatory flow for different kinematic viscosities of the fluid. More recently, several authors observed the disappearance of the ripples in Oscillating Water Tunnels (OWT) (Horikawa et al. 1982; Sawamoto and Yamashita 1986; Sato 1987; Dibajnia 1991). 
Table 3 summarizes the data sets compiled in the present study, where the type of flow motion (experimental set-up), the number of data points, the sediment properties (material used, relative density, median grain size), and the range of values for the main hydrodynamic parameters (critical wave orbital velocity at which the ripples start disappearing $U_{w, c r}$ and wave period, $T_{w}$ ). As Dibajnia (1991) noted, different definitions of the inception of sheet flow exist (e.g., disappearance of the ripples, modification of the energy dissipation) and, thus, induce some uncertainty in the experimental results depending on the definition used by the author(s).

Table 3. Summary of data sets on inception of sheet flow under oscillatory flow.

\begin{tabular}{|c|c|c|c|c|c|c|c|}
\hline Author(s) & Exp. & Number & Material & sec & $D_{50}, m m$ & $U_{w, c r}, \mathrm{~m} / \mathrm{sec}$ & $T_{\mathrm{w}}, \mathrm{sec}$ \\
\hline Manohar (1955) & OT & $\begin{array}{r}139 \\
17\end{array}$ & $\begin{array}{l}\text { Sand } \\
\text { Plastic }\end{array}$ & $\begin{array}{l}2.46-2.65 \\
1.05,1.28\end{array}$ & $\begin{array}{l}0.2-1.98 \\
3.17\end{array}$ & $\begin{array}{l}0.54-1.25 \\
0.32-0.73\end{array}$ & $\begin{array}{l}1.0-4.6 \\
2.9-9.3\end{array}$ \\
\hline $\begin{array}{l}\text { Chan et al. } \\
\text { (1972) }\end{array}$ & $\mathrm{HT}$ & $\begin{array}{r}3 \\
8 \\
25 \\
30 \\
16\end{array}$ & $\begin{array}{l}\text { Polystyrene } \\
\text { Cane sugar } \\
\text { Sand } \\
\text { Glass beads } \\
\text { Iron ore }\end{array}$ & $\begin{array}{l}1.32(1.4) \\
1.97(1.55) \\
1.97-2.55 \\
2.05-2.65 \\
3.95-5.10\end{array}$ & $\begin{array}{l}0.36 \\
0.25,0.50 \\
0.25-1.09 \\
0.09-0.50 \\
0.18-0.50\end{array}$ & $\begin{array}{l}0.23-0.29 \\
0.31-0.57 \\
0.33-0.86 \\
0.35-1.12 \\
0.63-1.73\end{array}$ & $\begin{array}{l}1.2-2.2 \\
0.8-2.3 \\
0.8-2.5 \\
0.8-2.5 \\
0.8-2.3\end{array}$ \\
\hline $\begin{array}{l}\text { Horikawa et al. } \\
\text { (1982) }\end{array}$ & OWT & $\begin{array}{l}17 \\
19\end{array}$ & $\begin{array}{l}\text { Sand } \\
\text { Plastic }\end{array}$ & $\begin{array}{l}2.66 \\
1.18-1.56\end{array}$ & $\begin{array}{l}0.22-0.70 \\
0.28-4.00\end{array}$ & $\begin{array}{l}0.56-1.15 \\
0.40-1.68\end{array}$ & $\begin{array}{l}3.5-7.0 \\
3.0-7.0\end{array}$ \\
\hline $\begin{array}{l}\text { Sawamoto and } \\
\text { Yamashita } \\
\text { (1986) }\end{array}$ & OWT & 4 & Sand, plastic & $2.65,1.58$ & $0.20-1.60$ & $0.20-1.01$ & 3.8 \\
\hline Sato (1987) & OWT & 3 & Sand & 2.65 & 0.18 & $0.47-0.56$ & $0.8-2.0$ \\
\hline Dibajnia (1991) & OWT & 18 & Sand & 2.65 & 0.20 & $0.62-0.96$ & $1.0-4.0$ \\
\hline
\end{tabular}

Manohar (1955) and Komar and Miller (1975) introduced similar criterion for the inception of sheet flow using a function, which included the waverelated mobility parameter $\Psi_{w}$, the wave-related Shields parameter $\theta_{w}$, and the grain-size Reynolds number $\Re *_{w}$, given as follows:

$$
\begin{aligned}
& \left\{\Psi_{w} \Re{ }^{1 / 2}\right\}_{c r, s f}=2000 \quad \text { (Manohar) } \\
& \left\{\theta_{w} \Re_{*_{w}}{ }^{1 / 3}\right\}_{c r, s f}=4.4 \quad \text { (Komar \& Miller) }
\end{aligned}
$$




$$
\text { with }\left\{\begin{aligned}
\Psi_{w} & =\frac{U_{w}^{2}}{(s-1) g d_{50}} \\
\theta_{w} & =\frac{\frac{1}{2} f_{w} U_{w}^{2}}{(s-1) g d_{50}} \\
\Re_{*} & =\frac{U_{w} d_{50}}{\nu}
\end{aligned}\right.
$$

where $U_{w}$ is the wave orbital velocity. Chan et al. (1972) investigated the effect of kinematic viscosity and relative particle density on the inception of sheet flow. They observed that wave period is of greater importance for the inception of sheet flow compared to what previous studies established. They arrived at the following relationship, introducing the Stokes boundary layer $\delta_{w}=\sqrt{v T_{w} / \pi}$ :

$$
\left\{\Psi_{w}\left(\frac{d_{50}}{\delta_{w}}\right)^{0.8}\right\}_{c r, s f}=43.6
$$

Sawamoto and Yamashita (1986) proposed a similar equation, but they modified the coefficient values (2/3 instead of 0.8 and 36 instead of 43.6).

Dibajnia (1991) developed a new formula based on the Chan et al. (1972) study. He introduced a parameter $\omega_{p l}$ for the inception of sheet flow defined as:

$$
\omega_{p l}=\frac{\frac{1}{2} U_{w}^{2}}{(s-1) g W_{s} T_{w}}
$$

The criterion proposed by Dibajnia (1991) may be written as follows:

$$
\omega_{p l, c r, s f}=10.6 \frac{d_{50}^{0.3} v^{0.2}}{W_{s}^{0.7} T_{w}^{0.5}}
$$

Dibajnia (1991) also investigated the effect of an asymmetric wave for which the maximum wave orbital velocity should be employed in applying Equation 55. 
You (1999) re-examined the Manohar (1955) data and proposed an iterative relationship for the critical orbital velocity that is a function of the scaled dimensionless immersed sediment weight $S_{*}=\sqrt{(s-1) g d_{50}^{3}} /(4 v)$ :

$$
U_{w, c r, s f}=\frac{v}{K_{1} d_{50}}\left(1-K_{2} \frac{d_{50} \omega}{U_{w, c r, s f}}\right)
$$

with $K_{1}=0.0134 S_{*}^{-0.78}$ and $K_{2}=287 S_{*}^{-0.59}$, and where $\omega=2 \pi / T_{w}$ is the angular frequency of the wave.

Comparison with laboratory data. Table 4 lists predictions of the critical orbital velocity within a factor of $1.25\left(P_{25}\right)$ of the measured values presented for the formulas discussed in the previous section ("factor of $\mathrm{x}$ " means between $\mathrm{x}$ times and $1 / \mathrm{x}$ times the measured critical orbital velocity $\left.U_{w, c r, s f, m e a s}\right)$. The results are given for all the data as well as for the Manohar (1955) data only (because many authors compared their results to this data set only). The table also presents the mean value of the difference $\Delta U_{w}=U_{w, c r, s f, p r e d}-U_{w, c r, s f, m e a s}$ and its standard deviation.

Table 4. Prediction of critical wave orbital velocity for inception of sheet flow within factor of 1.25 together with mean value and standard deviation of $\Delta U_{w}$.

\begin{tabular}{|l|l|l|l|l|l|l|}
\hline \multirow{2}{*}{ Author(s) } & \multicolumn{3}{|c|}{ All Data } & \multicolumn{4}{c|}{ Manohar Data } \\
\cline { 2 - 7 } & $P_{25}(\%)$ & $\overline{\Delta U_{w}}$ & $\operatorname{Std}\left(\Delta U_{w}\right)$ & $P_{25},(\%)$ & $\overline{\Delta U_{w}}$ & $\operatorname{Std}\left(\Delta U_{w}\right)$ \\
\hline Manohar (1955) & 67 & +0.05 & 0.31 & 92 & -0.04 & 0.11 \\
\hline Chan et al. (1972) & 43 & -0.11 & 0.28 & 28 & -0.21 & 0.13 \\
\hline Komar and Miller (1975) & 62 & -0.04 & 0.25 & 72 & -0.12 & 0.10 \\
\hline $\begin{array}{l}\text { Sawamoto and Yamashita } \\
\text { (1986) }\end{array}$ & 32 & -0.21 & 0.25 & 09 & -0.29 & 0.11 \\
\hline Dibajnia (1991) & 68 & +0.02 & 0.25 & 89 & +0.005 & 0.14 \\
\hline You (1999) & 66 & -0.12 & 0.70 & 92 & -0.05 & 0.10 \\
\hline Equation 57 & 76 & +0.004 & 0.25 & 96 & -0.05 & 0.11 \\
\hline
\end{tabular}

It appears that the Manohar (1955) and Dibajnia (1991) criteria yield the best overall results: $P_{25} \approx 65(90),\left|\Delta U_{w}\right| \leq 0.05(0.05)$ and $\operatorname{std}\left(\Delta U_{w}\right) \approx 0.3(0.1)$ (in brackets are the values for the Manohar data set only given). The Chan et al. (1972) and Sawamoto and Yamashita (1986) criteria (calibrated with their own data set) show reasonable overall 
behavior, but tend to underestimate the values from the Manohar data set. A similar comment can be made concerning the equation proposed by Komar and Miller (1975), even if their calibration was made using the Manohar data only. This could be a result of the expression used to compute the friction coefficient $\left(k_{s}=2 d_{50}\right.$ ) or because of the iterative approach to solve the equation (the friction factor $f_{w}$ is a function of the wave orbital velocity). The complexity of the equation proposed by You (1999) clearly illustrates the limits of fitting with a single data set (Manohar 1955): the equation predicts negative values on $U_{w, c r, s f}$ for some points in the Chan et al. (1972) data set.

Based on the Chan et al. (1972) study, the following expression is proposed:

$$
U_{w, c r, s f}=8.35 \sqrt{(s-1) g \sqrt{d_{50} \delta_{w}}}\left(1+r_{w}\right)
$$

where $r_{w}$ is the wave asymmetry coefficient $\left(r_{w}=u_{w, \max } / U_{w}-1\right.$, with $u_{w, \max }$ being the maximum wave velocity). Excluding the effect of the wave asymmetry $\left(r_{w}=0\right)$, Equation 57 may also be written similarly to the Chan et al. (1972) criterion:

$$
\left\{\Psi\left(\frac{d_{50}}{\delta_{w}}\right)^{0.5}\right\}_{c r, s f}=70
$$

Figure 17 shows a comparison between the observed critical wave orbital velocity $U_{w, c r, s f}$ for the inception of sheet flow and its predicted value using Equation 57. Improved agreement with the data is observed compared to the previous formulas. The overestimation of most of the values from the Chan et al. (1972) data may be attributable to the experiment arrangement. Chan et al. (1972) argued that the lower values they observed for the inception of the sheet flow regime may be due to the onset of turbulence in the tube used in their experiment. Horikawa et al. (1982) pointed out the effect of the sediment particle shape: the two groups of measurements from Horikawa et al. (1982) that are underestimated ( $U_{w, c r, s f, p r e d} /$ $\left.U_{w, c r, s, \text { meas }}<0.7\right)$ as well as the two groups of measurements from Chan et al. (1972) that are overestimated ( $U_{w, c r, s f, p r e d} / U_{w, c r, s f, \text { meas }}>2$ ) correspond to cylindrical-shaped plastic particles and spherical glass particles, respectively. A structure made of cylinders may be more "resistant" than a structure made of spheres, and thus tends not to move as readily. 


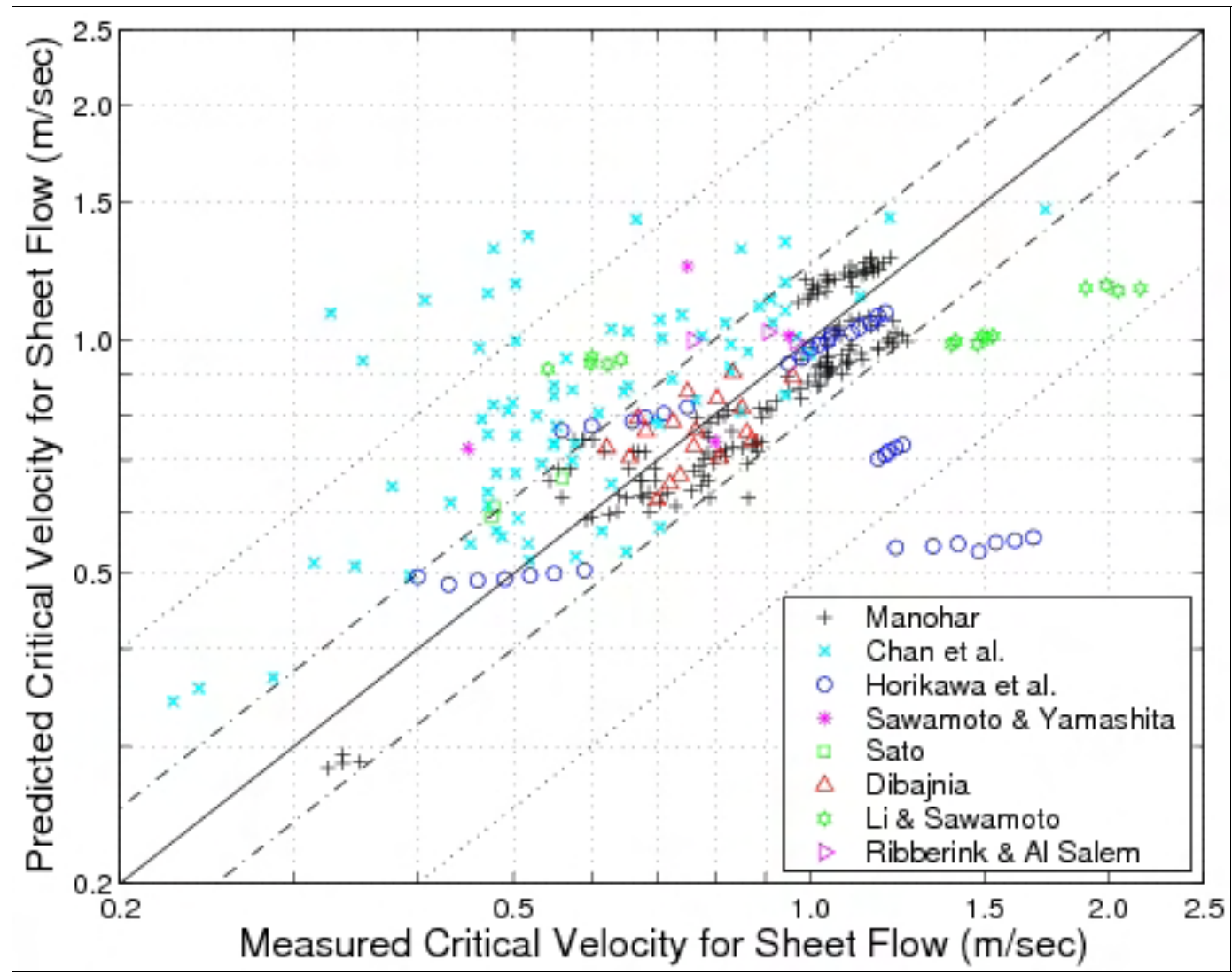

Figure 17. Comparison between observed critical wave orbital velocity $U_{w, c r, s f \text {,meas }}$ for inception of sheet flow and predicted value $U_{w, c r, s f, p r e d}$ using Equation 57. 


\section{Bed Load}

\section{Introduction}

Accurate prediction of sediment transport rates is an essential element in morphological studies of coastal, marine, and river environments. Sediment transport mainly occurs in two modes: bed load and suspended load. The bed load is the part of the total load that is traveling directly above the bed and is supported by intergranular collisions rather than fluid turbulence (Wilson 1966). The suspended load, on the other hand, is the part of the load which is primarily supported by the fluid turbulence (Fredsøe and Deigaard 1994). Thus, bed load includes mainly sediment transport for coarse materials (saltation) or fine material on plane beds (saltation at low shear stresses and sheet flow at high shear stresses), although both types of transport can occur together and the limit is not always easy to define. The earliest formulas (models) proposed to estimate bed-load transport were mainly based on the concept that the sediment transport rate can be related to the bottom shear stress (Meyer-Peter and Müller 1948; Einstein 1950) and these formulas were valid for steady, unidirectional flows. In coastal and marine environments, the process of sediment transport becomes increasingly complex due to the presence of oscillatory flows, and the interaction between steady and oscillatory flows. For example, in longshore sediment transport, the influence of the short waves is expressed through wave-induced sediment stirring that increases the bed shear stress (Bijker 1967, 1968; Watanabe 1982; Van Rijn 1993).

However, in the case of cross-shore sediment transport, a wave-averaged approach is not adequate because of the dominant role of the timedependent oscillatory orbital motion near the sea bed, induced by the short waves. For example, the residual cross-shore transport of sand due to short-wave asymmetry is generally described using an intrawave model concept, resolving the unsteady transport process through the wave cycle. Bagnold (1966) developed an "energetics transport model" in which the instantaneous transport through the wave cycle is related to the instantaneous energy dissipation rate due to bottom friction. Bagnold's (1966) approach has formed the basis for several bed-load transport models (Bailard and Inman 1981; Ribberink 1998) as well as total load transport models (Bailard 1981; Madsen and Grant 1976). These models use the 
bed-shear stress concept for river flow, and time-dependent transport through the wave cycle is treated in a quasi-steady way. That is, timehistory effects from previous phases of the wave cycle or from previous wave cycles are neglected.

The development of practical sediment transport models still has a strong empirical character and relies on physical insights and quantitative data obtained in laboratory and field studies. The objective of this study is to develop a reliable, robust, and general formula for predicting bed-load transport for a wide range of coastal, marine, and river conditions. Many data sets are incorporated in the model development including steady and unsteady (oscillatory) flows.

For the bed-shear stress concept, a major problem is to estimate the total shear stress at the bottom. As a first step in this study, only conduit, flume, and river data for steady flows over a plane bed were examined to implement the bed-load formula. For these kinds of experimental studies, the bed shear stress may be estimated from energy slope measurements. Then, the formula was generalized to oscillatory flows and combined steady and oscillatory flows. However, for these more complex flows, the bed shear stress cannot be estimated directly from the measurements, but the stress must be calculated from theoretical models of the bed roughness. Thus, comparisons with experimental data were made by means of the skin friction as proposed by Soulsby (1997) and a semi-empirical function to estimate the bottom roughness in the sheet flow regime from Wilson (1989a).

\section{Previous studies on bed-load transport under wave and current interaction}

The bed-load transport corresponds to the net flux near the bottom, which then includes bed load and sheet-flow transport. Many studies (analytic and experimental) have been performed for bed-load and sheet-flow transport, which are often related to the bottom shear stress.

This section presents several sediment transport formulas that include both current and wave effects. 
1. The Bijker (1968) and Van Rijn (1984a, b, 1989) formulas. The formulation of bed-load transport comes from a method used in the river environment and adapted to the coastal environment.

2. The Bailard (1981) bed-load formula, which involves an energetics description of sediment transport due to waves.

3. The Dibajnia and Watanabe (1992) sheet-flow formula, which is based on the instantaneous velocity taking into account wave and current interaction and the associated induced movement of sediment (calibrated with experiments on sheet-flow transport).

4. The Ribberink (1998) formula. Bed-load transport rate is estimated by integrating the instantaneous bed-load rate $q_{s b}(t)$ over a wave period.

\section{Bijker formula}

One of the first formulas for bed-load transport that is still often applied was proposed by Bijker $(1967,1968)$. It was derived from the Frijlink (1952) formula for current only with a modification of the bottom shear stress using a wave-current model. The direction of sediment fluxes is always that of the current because this formula is proposed to estimate the longshore transport rate. The Bijker formula is written:

$$
q_{s b}=C_{b} d \sqrt{\frac{\mu_{c} \tau_{c}}{\rho}} \exp \left[-0.27 \frac{\left(\rho_{s}-\rho\right) g d}{\mu_{c} \tau_{c w}}\right]
$$

where $q_{s b}$ is the sediment volume flux for bed load, $C_{b}$ a breaking wave parameter, $\mu_{c}$ the ripple parameter, $\tau_{c}$ the shear stress due to current only, and $\tau_{c w}$ the shear stress including the wave-current interaction.

The ripple parameter introduced by Bijker (1968) is defined by the following equation:

$$
\mu_{c}=\left(\frac{f_{c t}}{f_{c}}\right)^{3 / 2}
$$

where $f_{c t}$ is the total friction coefficient due to the current and $f_{c}$ the skin friction coefficient due to the current. The breaking wave coefficient is defined by the following relationships: 


$$
\begin{aligned}
& C_{b}=2 \quad \text { if } H_{w} / h<0.05 \\
& =2+3\left(H_{w} / h-0.05\right) \text { if } 0.05<H_{w} / h<0.4 \\
& =5 \quad \text { if } 0.4<H_{w} / h
\end{aligned}
$$

where $H_{w}$ is the wave height. The shear stress arising from the wavecurrent interaction is computed following the method proposed by Bijker (1968) where the effect of the waves is described through a stirring factor:

$$
\tau_{c w}=\left[1+0.5\left(\xi_{B} \frac{U_{w}}{U_{c}}\right)^{2}\right] \tau_{c}
$$

where $\xi_{B}=\sqrt{f_{w t} / f_{c t}}$ is a parameter for wave-current interaction and $f_{w t}$ the total friction coefficient due to waves.

\section{Bailard formula}

Bagnold (1966) introduced an energetics model for sediment transport, where the main idea is that the solid flux is proportional to the energy flux (local rate of energy dissipation):

$$
\begin{gathered}
q_{s b} \propto \Omega \\
\Omega=\frac{1}{2} \rho f_{c w}|\overrightarrow{u(t)}|^{3}
\end{gathered}
$$

where $\Omega$ is the energy flux from waves and currents, $f_{c w}$ the friction coefficient due to the waves and current combined, $u(t)$ the instantaneous velocity vector, in which $u(t)=U_{c}+u_{w}(t)$ with $U_{c}$ being the current velocity averaged over the depth and $u_{w}(t)$ the instantaneous wave velocity.

The Bailard and Inman (1981) formula was derived from Bagnold (1966) model concepts. It can take into account the effect of the instantaneous velocity profile from waves and current combined. For a horizontal bed, the formula can be written as a vector yielding the sediment volume transport:

$$
\vec{q}_{s b}=\frac{1}{2} \frac{f_{c w}}{g(s-1)} \frac{\varepsilon_{b}}{\tan \varphi}<|\vec{u}|^{2} \vec{u}>
$$


where $\varepsilon_{b}$ is the bed-load efficiency, and $<>$ represents the average over several wave periods. The bed-load efficiency is slightly different from the one given by Bagnold (1966). Bailard (1981) suggested based on calibration against field data that $\varepsilon_{b}=0.1$.

One difficulty concerning the formula is to estimate the friction coefficient for waves and current combined. Bailard did not specify any expression for the friction coefficient. Indeed, friction coefficients due to a current or to waves only display considerably different values $\left(f_{w} / f_{c} \approx 10-100\right)$. Madsen and Grant (1976) suggested the following relationship for the combined friction factor:

$$
f_{c w}=Y f_{c}+(1-Y) f_{w} \quad \text { with } Y=\frac{\theta_{c}}{\theta_{c}+\theta_{w}}
$$

where $\theta_{c}$ and $\theta_{w}$ are the Shields parameters due to current and waves, respectively.

\section{Van Rijn formula}

The Van Rijn (1989) formula is defined in the same way as the Bijker $(1967,1968)$ formula, and the bed-load transport rate is written as follows:

$$
q_{s b}=0.25 d_{50} d_{*}^{-0.3}\left(\frac{\tau_{c t}}{\rho}\right)^{0.5}\left(\frac{\tau_{c w}-\tau_{c r}}{\tau_{c r}}\right)^{1.5}
$$

where $\tau_{c t}=\mu_{f} \alpha_{c w} \tau_{c}$ the total shear stress due to current only (taking into account the influence of bed forms and waves), $\tau_{c r}$ the critical shear stress for sediment transport, $\mu_{f}=f_{c} / f_{c t}$ the shape factor, and $\alpha_{c w}$ a coefficient due to the presence of waves (which can affect the mean shear stress).

\section{Dibajnia and Watanabe formula}

Similar to the formulas of Bailard and Ribberink (discussed next), the Dibajnia and Watanabe (1992; see also Dibajnia 1995) formula divides the sediment transport into two half-cycles due to the presence of waves (Figure 18). During the first half-cycle, sediment moves in the direction of wave propagation, and then it moves in the opposite direction during the second half-cycle. An advantage of the formula is that it takes into account a possible quantity of sand still in suspension after each half-cycle that 


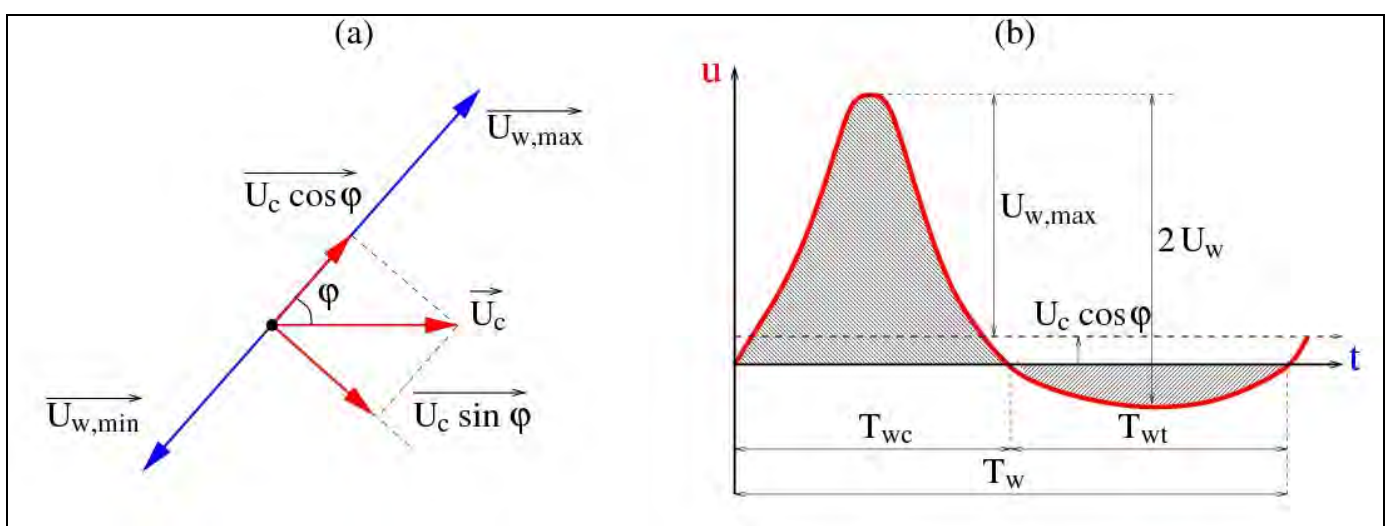

Figure 18. Definition of (a) wave and current directions, and (b) horizontal time-dependent velocity variation at bottom in direction of wave propagation (after Dibajnia and Watanabe 1992).

moves in the opposite direction. This phenomenon is referred to as a "phase lag," introduced by Dohmen-J anssen (1999). Another advantage is that the formula (similar to the Bailard (1981) and Ribberink (1998) formulas) allows for the description of nonlinear waves.

The solid volume flux is given by the following equation:

$$
\vec{q}_{s b}=A_{d w} W_{s} d \frac{\vec{\Gamma}}{\Gamma} \Gamma^{B_{d w}}
$$

where the coefficients of calibration are $A_{d w}=0.001$ and $B_{d w}=0.55$, and:

$$
\vec{\Gamma}=\frac{T_{w c} \vec{u}_{w c}\left(\Omega_{c}^{3}+\Omega_{t}^{\prime 3}\right)+T_{w t} \vec{u}_{w t}\left(\Omega_{t}^{3}+\Omega_{t}^{\prime 3}\right)}{\left(u_{w c}+u_{w t}\right) T_{w}}
$$

in which $T_{w}, T_{w c}, T_{w t}$ are the period and half-periods, respectively, of the wave taking into account the influence of the current (see Figure 18), $\Omega_{c}$ and $\Omega_{t}$ the amount of sand entrained and settled during the half-period $T_{w c}$ of the wave crest and $T_{w t}$ of the wave trough, respectively, $\Omega_{c}^{\prime}$ and $\Omega_{t}^{\prime}$ the amount of suspended sand remaining from the positive (crest) and the negative (trough) half-cycle, respectively, and $u_{w c}$ and $u_{w t}$ the quadratic velocity (wave and current combined) over each half-period calculated from: 


$$
u_{w j}^{2}=\frac{2}{T_{w j}} \int_{t}^{t+T_{w j}} u^{2}(t) d t+2 U_{c}^{2} \sin ^{2} \varphi
$$

where $j$ can be $c$ or $t, u(t)=U_{c} \cos \varphi+u_{w}(t)$, with $u_{w}(t)$ being the instantaneous wave orbital velocity, and $\varphi$ the angle between the wave and current directions (Figure 18). The nondimensional quantities in Equation 69 are defined as:

$$
\begin{aligned}
\text { if } \omega_{j} \leq \omega_{c r} \text { then } \Omega_{j} & =\omega_{j} \frac{2 W_{s} T_{w j}}{d} \\
\text { and } \quad \Omega_{j}^{\prime} & =0, \\
\text { if } \omega_{j} \geq \omega_{c r} \text { then } \Omega_{j} & =\frac{2 W_{s} T_{w j}}{d} \omega_{c r} \\
\text { and } \Omega_{j}^{\prime} & =\left(\omega_{j}-\omega_{c r}\right) \frac{2 W_{s} T_{w j}}{d}
\end{aligned}
$$

with:

$$
\omega_{j}=\frac{u_{w j}^{2}}{2(s-1) g W_{s} T_{w j}}
$$

where $j$ can be $c$ or $t$ as before.

The parameter $\omega_{c r}$ characterizes the ripples (a function of the total shear stress including waves and current):

$$
\begin{aligned}
& \omega_{c r}=0.03 \quad \text { if } \theta_{c w, \max } \leq 0.2 \\
& =1-0.97\left[1-\left(\frac{\theta_{c w, \max }-0.2}{0.4}\right)^{2}\right]^{0.5} \text { if } 0.2<\theta_{c w, \max }<0.6 \\
& =1 \quad \text { if } 0.6<\theta_{c w, \max }
\end{aligned}
$$

where $\theta_{c w, \max }$ is the maximum Shields parameter due to waves and current combined (following Soulsby 1997, pp. 87-95).

\section{Ribberink formula}

Ribberink (1998) proposed a model for the quasi-steady bed-load transport for which the instantaneous solid flux is assumed to be a function of 
the difference between the actual time-dependent bed shear stress and the critical bed shear stress (Figure 19). This formula has been calibrated against flume data for waves and current combined in the plane-bed mode (suspended load negligible), as well as for field data for unidirectional flow in rivers.

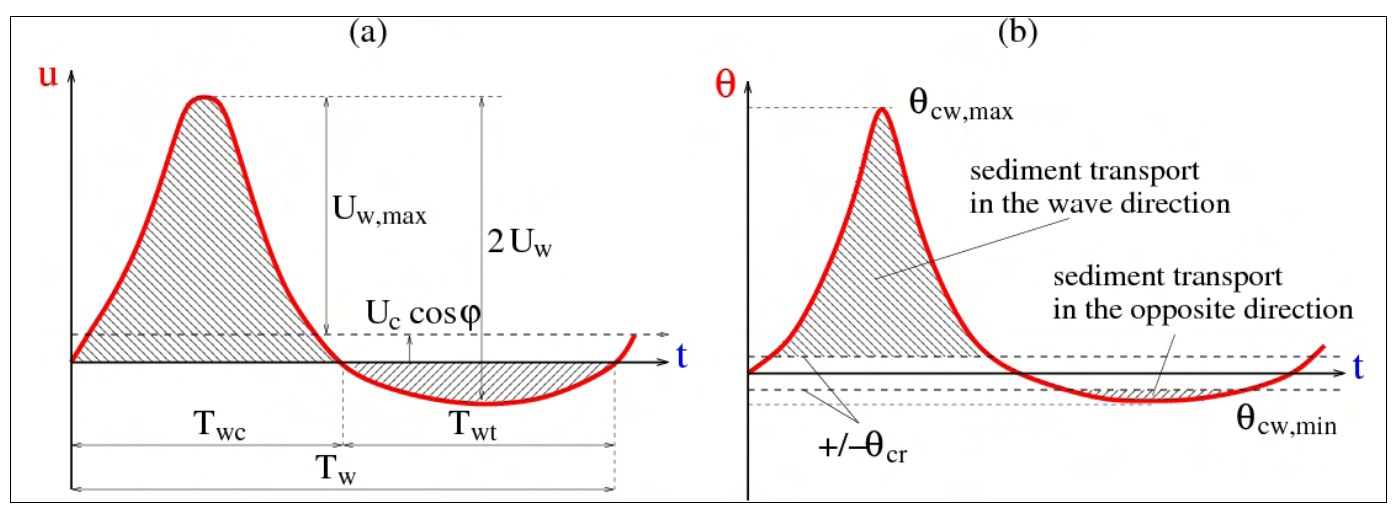

Figure 19. Time variation of bottom velocity (a) in wave direction, and (b) induced shear stress for waves and current combined (after Ribberink 1998).

Thus, Ribberink (1998) proposed the following transport formula:

$$
\vec{q}_{s b}=m_{R i b} \sqrt{(s-1) g d^{3}}<\left(|\overrightarrow{\theta(t)}|-\theta_{c r}\right)^{n_{R i b}} \frac{[\overrightarrow{\theta(t)}]}{|\theta(t)|}
$$

where $\vec{\theta}(t)=0.5 f_{c w}|u(t)| \vec{u}(t) /[(s-1) g d]$ is the time-dependent Shields parameter (Figure 19) with the instantaneous velocity $\vec{u}(t)=\vec{U}_{c}+\vec{u}_{w}(t)$, the wave-current friction factor $f_{c w}$ computed according to Madsen and Grant (1976), $\theta_{c r}$ the critical Shields parameter, < > the time-average over several wave periods, and $m_{R i b}=11, n_{R i b}=1.65$ calibration coefficients. Ribberink (1998) proposed to compute the total roughness from $k_{s t}=\max \left\{k_{s} ; d\left[1+6\left(<|\theta(t)|>/ \theta_{c r}-1\right)\right]\right\}$, where $k_{s}$ is the Nikuradse skin roughness.

\section{Bed-load transport by currents}

Here, bed load refers mainly to the rolling, sliding, and jumping grains in almost continuous contact with the bed. Also, it follows the ideas of Wilson (1966) about upper-regime transport where a layer with a thickness of 
several grain diameters is transported and where intergranular collision is important (sheet flow).

\section{Existing formulas}

The bed-load transport is often represented by the following nondimensional parameter:

$$
\Phi=\frac{q_{s b}}{\sqrt{(s-1) g d_{50}^{3}}}
$$

where $q_{s b}$ is the volumetric bed-load sediment transport rate per unit time and width. Some authors have also proposed to use the parameter $\Phi_{b}=q_{s b} /\left(W_{s} d_{50}\right)$. If $d_{50}>10^{-3} \mathrm{~mm}, \Phi_{b} \approx \Phi$ because the settling velocity is proportional to the square-root of the median diameter (according to Van Rijn 1984a), whereas for $d_{50}<10^{-3} \mathrm{~mm}, \Phi_{b}$ becomes smaller than $\Phi$, implying that $\Phi_{b}$ introduces a characterization of the sediment transport rate that is more sensitive for fine sediments.

Several relationships for estimating the bed-load sediment transport under a steady current have been proposed where the rate is related to the dimensionless bottom shear stress or Shields parameter:

$$
\theta_{c}=\frac{\tau_{c}}{\left(\rho_{s}-\rho\right) g d_{50}}=\frac{\frac{1}{2} f_{c} U_{c}^{2}}{(s-1) g d_{50}}
$$

Here, $\tau_{c}$ is the shear stress at the bottom due to the current. Three commonly applied formulas were investigated in this study:

$$
\begin{gathered}
\text { Meyer-Peter \&Müller (1948) } \Phi=8\left(\theta_{c}-\theta_{c r}\right)^{3 / 2} \\
\text { Nielsen (1992) } \Phi=12 \theta_{c}^{0.5}\left(\theta_{c}-\theta_{c r}\right) \\
\text { Ribberink (1998) } \Phi=11\left(\theta_{c}-\theta_{c r}\right)^{1.65}
\end{gathered}
$$

where $\theta_{c r}$ is the critical Shields parameter. The formula for $\theta_{c r}$ proposed by Soulsby and Whitehouse (1997; see also Soulsby (1997), p. 104-110) was used in the present study (Equation 49). 


\section{Comparison with data}

To investigate bed-load transport formulas, available data sets on sediment transport covering a wide range of unidirectional steady currents were compiled and analyzed, including both laboratory and field data.

Table 5 summarizes these data sets with the type of flow motion and sediment properties listed. It may be noted that many data sets come from the compilation made by Brownlie (1981), where limited information was given about the type of sediment transport (bed load and/ or suspended load), although comments on the presence of bed forms were provided. From these data, only plane-bed cases were selected, where bed load should prevail. Typically, for fine sediment, suspended load is not negligible if bed forms appear. Thus, bed-load transport in the presence of bed forms was not investigated. The data base compiled covers a wide range of median grain sizes, many of them considerably coarser than what would be found at a coastal inlet, but the range provides an informative test on the limits of the formulas. Also, the currents included in the data base covered a broad range. Figures 20a and 20b display histograms of the median grain size and mean current speed for the data base employed.

Table 5. Data base compiled to study bed-load sediment transport in steady current (plane bed cases only).

\begin{tabular}{|l|l|l|l|l|l|}
\hline Author(s) & Flow Type & Number & Material & S & $D_{50}(\mathrm{~mm})$ \\
\hline Gilbert (1914) & $\begin{array}{l}\text { Steady uniform flow, } \\
\text { flume, plane bed }\end{array}$ & 250 & Sand & 2,65 & $0.3-4.9$ \\
\hline $\begin{array}{l}\text { U.S. Army Engineer } \\
\text { Waterways Experiment } \\
\text { Station (1935-1936) } \\
\text { (in Brownlie 1981) }\end{array}$ & $\begin{array}{l}\text { Steady uniform flow, } \\
\text { flume, plane bed }\end{array}$ & 162 & Sand & 2.65 & $0.18-4.1$ \\
\hline Willis et al. (1972) & Flume, plane bed & 43 & Sand & 2.65 & 0.1 \\
\hline Brownlie (1981) & $\begin{array}{l}\text { Various experimental data } \\
\text { Plane bed }\end{array}$ & 297 & $\begin{array}{l}\text { Plastic } \\
\text { Sand }\end{array}$ & $\begin{array}{l}1.30-1.41 \\
2.49-2.67\end{array}$ & $\begin{array}{l}2.2-20.2 \\
0.088-20\end{array}$ \\
\hline Brownlie (1981) & $\begin{array}{l}\text { Various field data, plane } \\
\text { bed }\end{array}$ & 40 & Sand & 2.65 & $0.84-7.0$ \\
\hline $\begin{array}{l}\text { Smart (1984, 1999 } \\
\text { with Nikora and Smart } \\
\text { 1997) }\end{array}$ & $\begin{array}{l}\text { Exp. for steep channels } \\
\text { and field data, plane bed }\end{array}$ & 140 & $\begin{array}{l}\text { Sand } \\
\text { Gravel }\end{array}$ & $\begin{array}{l}2.65 \\
2.65\end{array}$ & $\begin{array}{l}2.0-10.5 \\
53-200\end{array}$ \\
\hline $\begin{array}{l}\text { Nnadi and Wilson } \\
\text { (1992) }\end{array}$ & $\begin{array}{l}\text { Pressurized closed } \\
\text { conduit }\end{array}$ & 105 & $\begin{array}{l}\text { Bakelite } \\
\text { Sand } \\
\text { Nylon }\end{array}$ & $\begin{array}{l}1.55 \\
2.67 \\
1.14\end{array}$ & $\begin{array}{l}0.67-1.05 \\
0.70 \\
3.94\end{array}$ \\
\hline
\end{tabular}




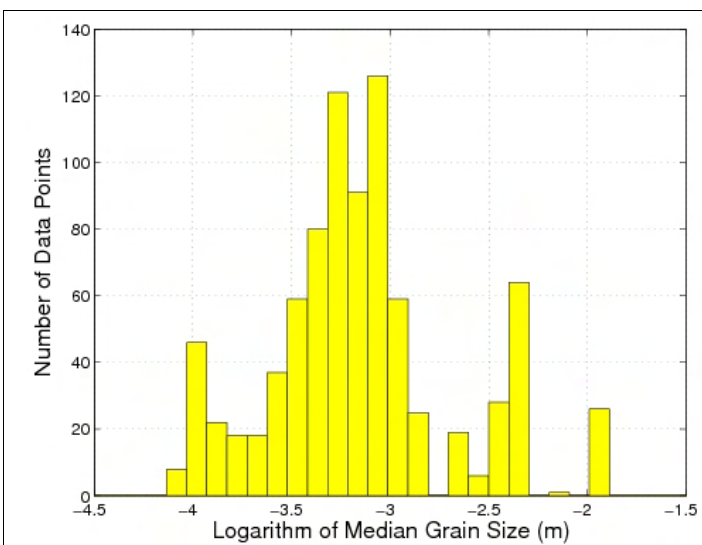

(a)

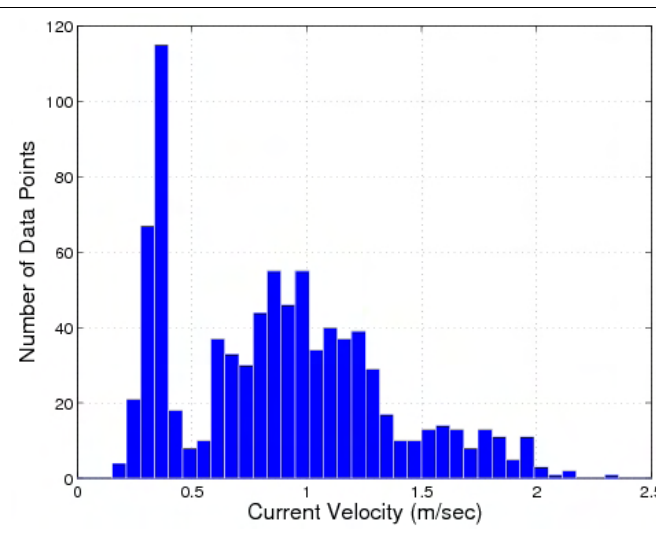

(b)

Figure 20. Distribution of median grain size and mean current speed for database compiled on sediment transport under steady current.

The existing formulas selected for comparison with the data relate the sediment transport rate to the bed shear stress, and they were developed for bed-load transport. In the employed database, it is uncertain what the ratio was between bed load and suspended load in each individual case. For the cases with coarser sediment, bed load must have dominated, with the grains moving through rolling, sliding, and jumping for lower shear stresses or in a sheet-flow layer for higher shear stresses. Even where suspended load might have been significant, experience with the Watanabe (1989) formula shows that the total load can often be related to the bed shear stress. This implies that the tested formulas may have some predictive potential in such a situation, although it is in principle outside their range of applicability.

Figure 21 illustrates a comparison between the sediment transport rate calculated with the Meyer-Peter and Müller (1948) formula and the compiled database. The other studied formulas yield similar agreement and exhibit the same general behavior, although the Nielsen (1992) formula showed slightly less agreement with the data. From Figure 21, two observations can be made: (1) for the Willis et al. (1972) data, the formulas underpredict the transport rate, which is probably due to the fine sediment used in the experiment and probable significant transport in suspension that the formulas fail to accurately quantify; and (2) for small transport rates, close to $\theta_{c r}$, the formulas overpredict the transport rate. With regard to the latter observation, it should be noted that all individual cases where the predictions implied a zero transport because $\theta_{c}<\theta_{c r}$ were not plotted in the figure because of the log-log scale. 


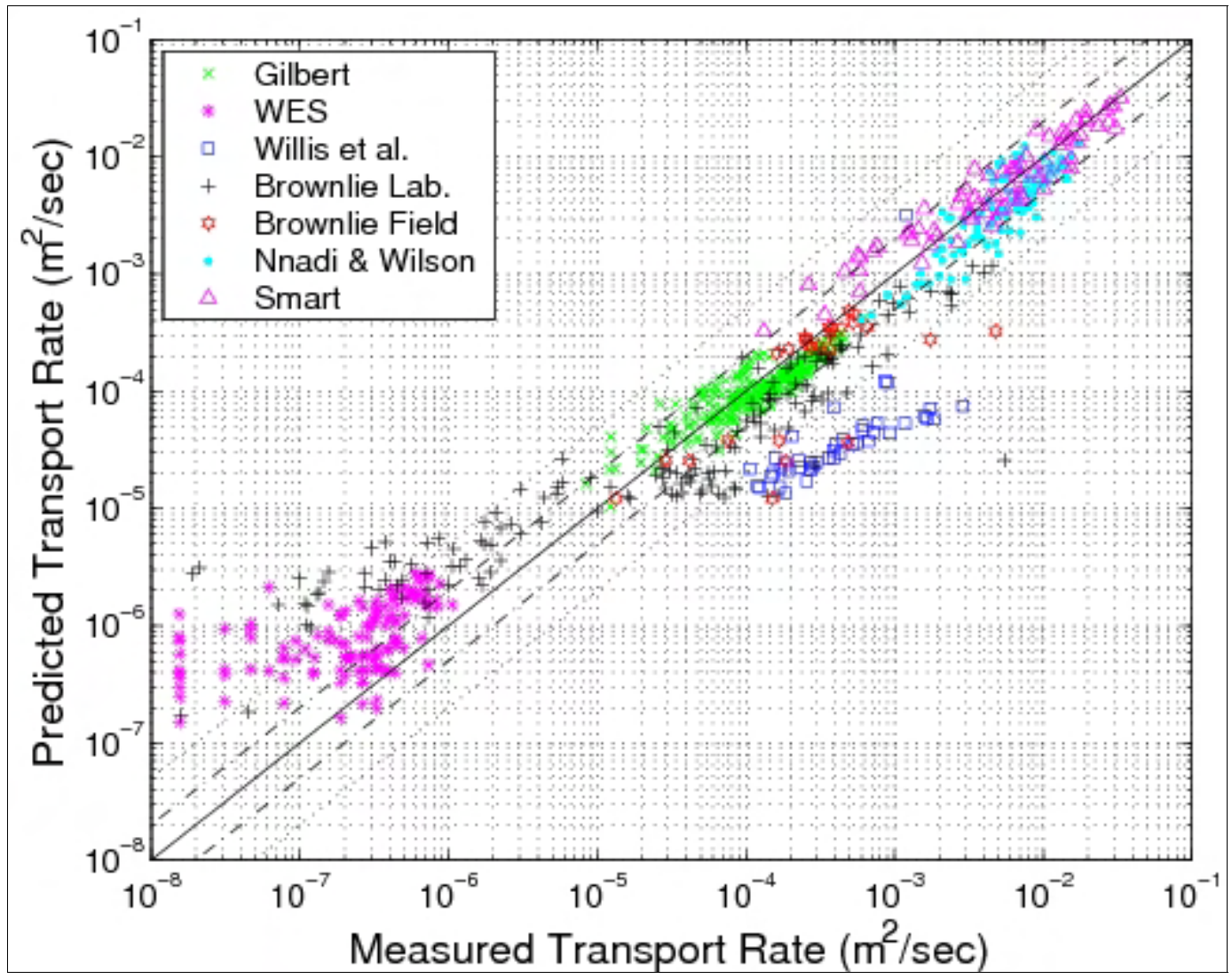

Figure 21. Comparison between Meyer-Peter and Müller formula and compiled database on sediment transport rates.

Because marked uncertainties exist in the measurements of the bed-load sediment transport (especially for field measurements), a prediction within a factor 2 of the measurements is considered to be a satisfactory result. In Table 6, the percentage of predicted values included within a factor of 2 or a factor of 5 deviation is presented (Px2 and Px5, respectively), as well as the root-mean-square error defined as:

$$
E_{\text {rms }}=\frac{1}{n} \sum\left[\log \left(\frac{q_{s b, \text { pred }}}{q_{s b, \text { meas }}}\right)\right]^{2}
$$

To avoid any distortion in the calculation of $E_{r m s}$ for low transport rates (where $q_{s b, \text { pred }}$ or $q_{s b \text {,meas }}$ could be found equal to zero), a minimum value for $q_{s b \text {,pred }}$ and $q_{s b \text {,meas }}$ was specified $\left(q_{s b, \text { min }}=10^{-8} \mathrm{~m}^{2} / \mathrm{sec}\right)$. It appears that the Meyer-Peter and Müller (1948) formula, which was originally calibrated with coarse sediment data, gives fairly good results in comparison to the measurements, even for large shear stresses and fine sediment. 
Table 6. Prediction of bed-load transport rate within factor of 2 and 5 of measured values and root-mean-square errors using current only data.

\begin{tabular}{|l|l|l|l|l|l|l|}
\hline \multirow{2}{*}{ Author(s) } & \multicolumn{3}{|c|}{ All Data } & \multicolumn{3}{c|}{ All Data Except Willis et al. } \\
\cline { 2 - 8 } & Px2 (\%) & Px5 (\%) & $E_{\text {rms }}$ & Px2 (\%) & Px5 (\%) & $E_{\text {rms }}$ \\
\hline Meyer-Peter and Müller & 62 & 83 & 0.34 & 66 & 87 & 0.30 \\
\hline Nielsen & 54 & 72 & 0.48 & 57 & 75 & 0.46 \\
\hline Ribberink & 66 & 85 & 0.28 & 69 & 89 & 0.25 \\
\hline Equation 81 & 73 & 89 & 0.19 & 78 & 93 & 0.15 \\
\hline
\end{tabular}

An underestimation is, however, observed for the Willis et al. (1972) data, but as sediment used in this experiment was very fine $(0.1 \mathrm{~mm})$, some suspended transport probably occurred. The three formulas studied show similar behavior for large shear stresses with a slightly better prediction skill for the Ribberink (1998) formula. For smaller Shields parameter ( $\theta_{c}$ close to $\theta_{c r}$, where $\theta_{c r}$ is estimated using the Soulsby and Whitehouse (1997) formula), all formulas tend to overestimate the bed-load rate with errors up to one order of magnitude for the Nielsen (1992) formula, which significantly increases the $E_{r m s}$-value. Thus, it seems that the use of the critical Shields parameter as a limit for specifying no transport is not sufficiently accurate for the existing formulas.

\section{New formula for bed-load transport}

To better understand the influence of the critical value of the Shields parameter on the sediment transport, $\Phi$-values from the measurements are plotted versus the ratio $\theta_{c} / \theta_{c r}$ in Figure 22. The studied formulas are also plotted with a constant value for $\theta_{c r}=0.04$ (mean value for the data set).

All the predictive formulas are in fairly good agreement with the data if $\theta_{c}>5 \theta_{c r}$, but they tend to slightly overestimate $\Phi$ when $\theta_{c r}<\theta_{c}<5 \theta_{c r}$. For this range of values, the Meyer-Peter and Müller (1948) and Ribberink (1998) formulas exhibit a better behavior than the Nielsen (1992) formula. 


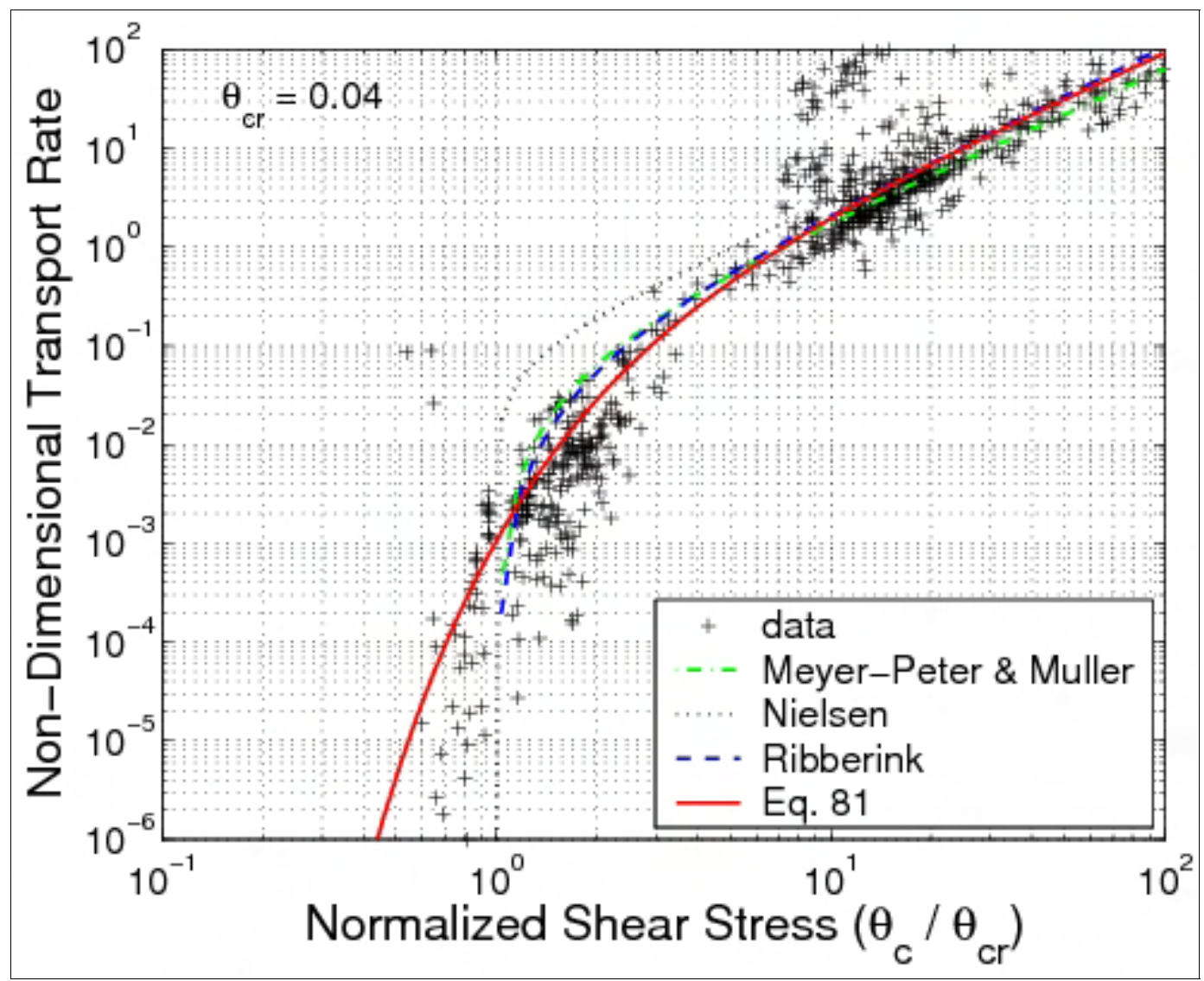

Figure 22. Effect of critical Shields parameter on bed-load transport rate: comparison between data and studied formulas.

However, if $\theta_{c} \lesssim \theta_{c r}$, significant deviations in the predictions occur. All these formulas predict no sediment transport, although small sediment transport is often observed. The prediction of the critical Shields parameter is obviously associated with marked uncertainty (so is the sediment transport estimation for these low values). Thus, to avoid such errors, a new approach is introduced by including an exponential relationship for the effect of the critical Shields parameter, which allows low sediment transport rates if $\theta \approx \theta_{c r}$. Calibration against the measurements led to the following bed-load formula:

$$
\Phi=12 \theta_{c}^{1.5} \exp \left(-4.5 \frac{\theta_{c r}}{\theta_{c}}\right)
$$

Cheng (2002) proposed a formula similar to Equation 81, but without taking into account the effect of a varying critical Shields parameter. The Cheng (2002) formula seems to include a constant value of $\theta_{c r} \approx 0.05$. The 
effect of a varying critical value of the Shields parameter is, however, not negligible as observed by Meyer-Peter and Müller (1948).

Figures 22, 23, and 24 indicate that this new approach of describing the effect of the critical Shields parameter significantly improves the prediction skill for weak shear stresses. Predictions within a factor- 2 deviation reach 70 percent (even 80 percent without the Willis et al. (1972) data), which increase the agreement by 10 percent compared to previous formulas (Table 6). The new relationship tends to overestimate the transport rate if $\theta_{c}$ is much smaller than $\theta_{c r}$ (see Figure 22), but reproduces trends well for small measured rates.

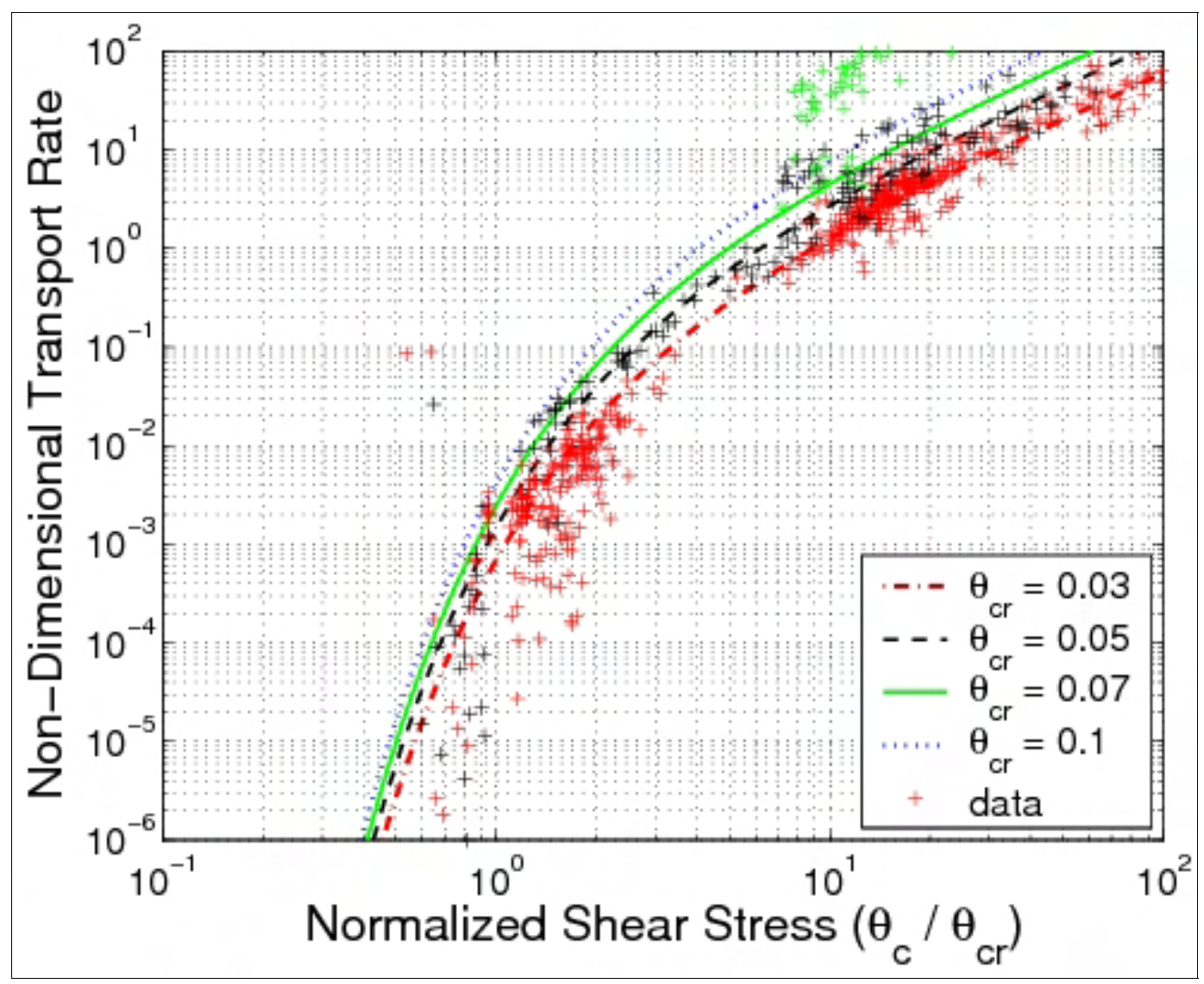

Figure 23. Influence of critical Shields parameter on bed-load transport rate illustrated through data and Equation 81 (for different values on $\theta_{c r}$ ).

Figure 24 plots measured and calculated transport rate values for the database using the new formula (Equation 81). The plot confirms the improvement in the prediction of the bed-load transport. 


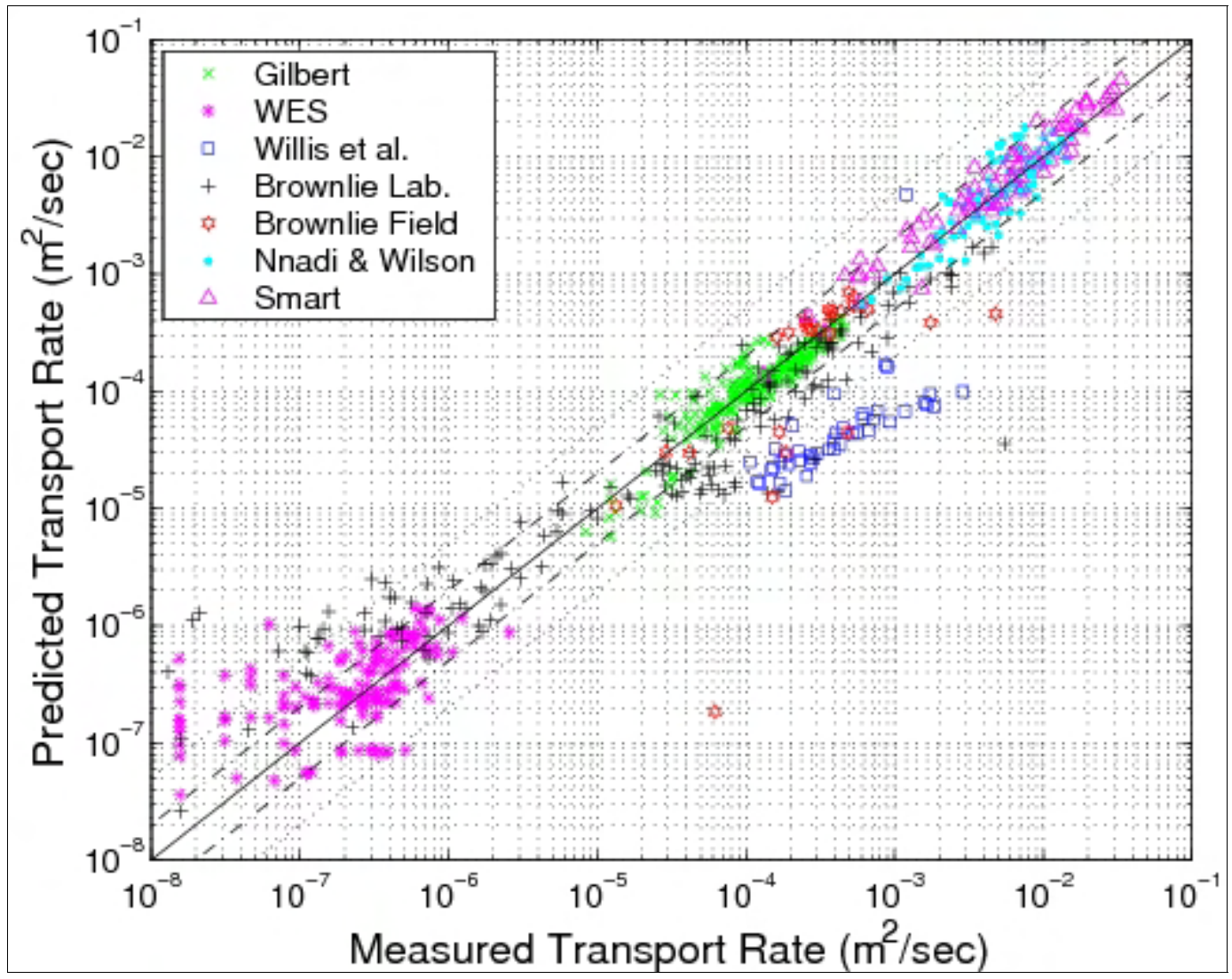

Figure 24. Comparison between bed-load transport for current only predicted by new formula (Equation 81) and measurements.

\section{Bed-load transport by waves}

\section{Existing formulas}

Several relationships for bed-load transport by waves have been proposed that relate the transport rate to the wave orbital velocity at the bottom $U_{w}$ or the wave Shields parameter $\theta_{w}$ defined as:

$$
\theta_{w}=\frac{\frac{1}{2} f_{w} U_{w}^{2}}{(s-1) g d_{50}}
$$

where $f_{w}$ is the dimensionless wave friction factor. Assuming that the rough turbulent regime is fully developed, the friction factor can be estimated, for example, with the formula suggested by Swart (1974) (see Equation 25). 
Transport formulas have been employed to estimate the wave half-cycle sediment transport $\Phi_{1 / 2}$ (Madsen and Grant 1976; Soulsby et al. 1993; Soulsby 1997). The net transport can then be calculated as the difference between the half-cycle transport beneath the crest and beneath the trough (the shear stress is calculated using the maximum and minimum values of the wave velocity at the bottom, $u_{w, \max }$ and $u_{w, \min }$, respectively, instead of the wave orbital velocity $U_{w}$ ). In some other studies (Bailard and Inman 1981; Ribberink 1998), a relationship based on the instantaneous wave velocity was introduced for the bed load $\Phi(t)$ that can be integrated over a wave period. The following formulas are among the most common ones for calculating sediment transport under waves:

$$
\text { Madsen \& Grant (1976) } \quad \Phi_{1 / 2}=12.5 \frac{W_{s}}{\sqrt{(s-1) g d_{50}}} \theta_{w}^{3}
$$

Bailard \&Inman (1981) $\Phi(t)=\frac{\varepsilon_{b} f_{w}}{(s-1)^{2} g^{2} \tan \phi d_{50}}\left|u_{w}(t)\right|^{2} u_{w}(t)$

Dibajnia \&Watanabe (1992) $\Phi=0.001 \frac{W_{s}}{\sqrt{(s-1) g d_{50}}} \Gamma^{0.55} \frac{\Gamma}{|\Gamma|}$

Soulsby (1997) $\Phi_{1 / 2}=5.1\left(\theta_{w}-\theta_{c r}\right)^{3 / 2}$

$$
\text { Ribberink (1998) } \Phi(t)=11\left(\left|\theta_{w}(t)\right|-\theta_{c r}\right)^{1.65} \frac{\theta_{w}(t)}{\left|\theta_{w}(t)\right|}
$$

It should be noted that Madsen and Grant (1976) as well as Dibajnia and Watanabe (1992) used the parameter $\Phi_{b}$ instead of the parameter $\Phi$ (see previous discussion), which explains the coefficient $W_{s} / \sqrt{(s-1) g d_{50}}$ in their formulas. Bailard and Inman (1981) proposed a coefficient value of $\varepsilon_{b}=0.13$, and $\phi$ is the internal friction angle of the sediment ( $\phi \approx 30 \mathrm{deg}$ ). Dibajnia and Watanabe (1992) defined $\Gamma$ as a function of the half periods $T_{w c}$ and $T_{w t}$ and the amount of sediment entrained that settle in each halfperiod, because some of the sediment might still be in suspension from the previous half-period (see also Camenen and Larroudé 2003). 


\section{Development of new formula}

As Madsen (1991) and Ribberink (1998) proposed, the instantaneous sediment transport rate may be related to the instantaneous shear stress in the same manner as for the steady case. Following the idea of Dibajnia and Watanabe (1992), a simplified velocity variation at the bottom may be considered to estimate the effect of the wave asymmetry on the sediment transport. Thus, the net sediment transport over a wave period is estimated for each half-period using a characteristic value for the quadratic velocity, or equivalently on the shear stress (if the friction coefficient is assumed to be constant). Thus, the mean value of the instantaneous shear stress over half a period may be used (Figure 25):

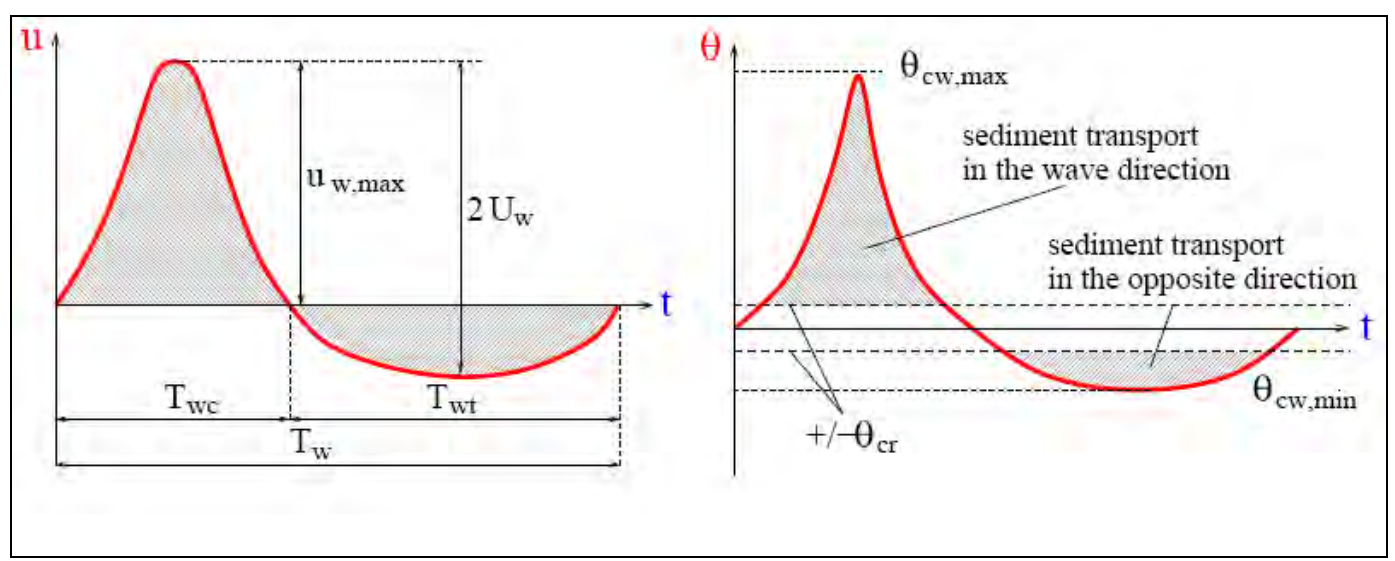

Figure 25. (a) Typical wave velocity variation, and (b) instantaneous Shields parameter variation over wave period in direction of wave propagation.

$$
\begin{aligned}
& \theta_{w, \text { onshore }}=\frac{1}{T_{w c}} \int_{0}^{T_{w c}} \theta_{w}(t) d t \\
& \theta_{w, \text { ofshore }}=\frac{1}{T_{w t}} \int_{T_{w c}}^{T_{w}} \theta_{w}(t) d t
\end{aligned}
$$

where $T_{w c}$ and $T_{w t}\left(=T_{w}-T_{w c}\right)$ are the half periods where the instantaneous velocity $u_{w}(t)$ (or instantaneous Shields parameter) is onshore $\left(u_{w}(t)>0\right)$ or offshore $\left(u_{w}(t)<0\right)$, respectively, and the instantaneous shear stress is defined as follows:

$$
\theta_{w}(t)=\frac{\frac{1}{2} f_{w}\left|u_{w}(t)\right| u_{w}(t)}{(s-1) g d_{50}}
$$


Figure 25 presents a typical velocity variation and the associated instantaneous Shields parameter variation over a wave period. In the case of an asymmetric wave, a maximum in the shear stress occurs during onshore flow (in the direction of the wave propagation) that is larger than the minimum during offshore flow, which induces a net sediment transport in the direction of the waves.

A constant value on the friction coefficient over the wave period was assumed in calculating the Shields parameter (Equation 89). Drake and Calantoni (2001) and Antunes Do Carmo et al. (2003) showed that the wave friction coefficient depends also on the acceleration of the fluid near the bottom. This dependence was previously noted by Trowbridge and Madsen (1984a, b), who made an analytical study of the turbulent wave boundary layer, highlighting the contribution of a time-varying eddy viscosity. However, it is assumed here that the predictions of the shear stresses using a constant friction factor are sufficiently accurate for application in practical sediment transport calculations.

Using the same approach as for the steady current, an equation for the net sediment transport under waves may be derived similar to Equation 81. The net sediment transport under waves is expressed as:

$$
\Phi=a_{w} \sqrt{\theta_{w, \text { onshore }}+\theta_{w, \text { offshore }}} \theta_{w, m} \exp \left(-b_{w} \frac{\theta_{c r}}{\theta_{w}}\right)
$$

where $a_{w}$ and $b_{w}$ are coefficients $\left(\left|\theta_{w, \text { onshore }}\right|\right.$ is always supposed to be larger than $\left.\left|\theta_{w, \text { offshore }}\right|\right)$ and $\theta_{w, m}=<\left|\theta_{w}(t)\right|>$ is the time-averaged absolute value of the instantaneous Shields parameter.

A conceptual model that supports this type of formulation would be based on the following assumptions:

- The transport in the bottom layer is the product of the typical speed of the layer and the layer thickness, where the former is denoted $U_{s}$ and the latter $\delta_{m}$.

- The speed $U_{s}$ is assumed proportional to the net shear velocity over a wave period, which gives a dependence on $\left|\theta_{w, \text { onshore }}+\theta_{\text {offshore }}\right|^{1 / 2}$ $\left(u_{*} \propto \theta^{1 / 2}\right)$. 
- $\delta_{m}$ is assumed proportional to the mean wave shear stress, which gives the dependence on $\theta_{w, m}$.

The overall effect of the critical shear stress on the sediment transport over a wave period is estimated using the same approximation as for a steady current. However, because Soulsby (1997, pp. 104-106) proposed to compute the critical shear stress based on the maximum shear stress, the maximum Shields parameter is used in Equation 90.

A difficulty encountered in applying the formula (and any other formulas based on the shear stress) is to estimate the total Shields parameter (or total friction coefficient) for the sheet flow mode. The work by Wilson (1989a) on wave-induced sheet-flow roughness was employed in this study, where he proposed to use the same equation as for steady current, but with the maximum wave-induced Shields parameter $\left(k_{s}=k_{s, \mathrm{sfw}}=5 \theta_{w}\right.$ $d_{50}$ ). Because most of the results presented by Ribberink (1998) were based on the skin friction, to be consistent, two computations were made, first using the skin friction ( $k_{s}=2 d_{50}$ ) and then the Wilson formula (Equation 41). It should be noted that the Wilson formula requires an iterative approach in solving for $k_{s}$.

\section{Comparison with experimental data}

To investigate bed-load transport under waves only, a wide range of existing data sets was compiled and analyzed. Table 7 summarizes these data sets, in which the type of experiment, sediment properties, and wave properties are listed. It can be observed that most of the data are from oscillating water tunnels (OWT). The OWT experiments have two advantages for this type study: large orbital velocities can be achieved, and bedload transport prevails. Previously, experimental studies were often carried out using an oscillating tray (OT: oscillating bed in a tank of still water; Manohar 1955; Kalkanis 1964; Abou-Seida 1965; and Sleath 1978). More recently, Dohmen-J anssen and Hanes (2002) carried out an experiment in a large wave flume (LWF). 
Table 7. Data summary for bed-load sediment transport experiments carried out in oscillatory flow with and without current.

\begin{tabular}{|c|c|c|c|c|c|c|c|c|}
\hline Author(s) & \begin{tabular}{|l} 
Exp. \\
Facil.
\end{tabular} & Cycle & Number & $s$ & $\begin{array}{l}d_{50} \\
(\mathrm{~mm})\end{array}$ & $U_{c}(\mathrm{~m} / \mathrm{sec})$ & $\begin{array}{l}U_{w, \max } \\
(\mathrm{m} / \mathrm{sec})\end{array}$ & $\begin{array}{l}T_{w} \\
(\mathrm{sec})\end{array}$ \\
\hline Kalkanis (1964) & OT & Half & 25 & 2.63 & 1.68-2.82 & 0 & $0.28-0.71$ & $3.2-6.2$ \\
\hline $\begin{array}{l}\text { Abou Seida } \\
\text { (1965) }\end{array}$ & OT & Half & $\begin{array}{l}34 \\
9\end{array}$ & $\begin{array}{l}2.65 \\
2.23\end{array}$ & $\begin{array}{l}0.14-2.61 \\
0.70\end{array}$ & $\begin{array}{l}0 \\
0\end{array}$ & $\begin{array}{l}0.35-1.28 \\
0.41-0.80\end{array}$ & $\begin{array}{l}1.7-5.1 \\
2.0-4.8\end{array}$ \\
\hline Sleath (1978) & OT & Half & $\begin{array}{l}22 \\
12\end{array}$ & \begin{tabular}{|l|}
2.60 \\
1.138
\end{tabular} & $\begin{array}{l}1.89,4.24 \\
3.04\end{array}$ & $\begin{array}{l}0 \\
0\end{array}$ & $\begin{array}{l}0.20-0.68 \\
0.07-0.17\end{array}$ & $\begin{array}{l}0.5-2.7 \\
1.3-9.0\end{array}$ \\
\hline $\begin{array}{l}\text { Horikawa et al. } \\
\text { (1982) }\end{array}$ & OWT & Half & 6 & 2.66 & 0.2 & 0 & $0.76-1.27$ & $2.6-6.0$ \\
\hline $\begin{array}{l}\text { Sawamoto and } \\
\text { Yamashita } \\
(1986)\end{array}$ & OWT & Half & 22 & $\begin{array}{l}1.58 \\
2.65\end{array}$ & $\begin{array}{l}1.5 \\
0.2-1.8\end{array}$ & $\begin{array}{l}0 \\
0\end{array}$ & $\begin{array}{l}0.74-1.25 \\
0.46-1.25\end{array}$ & $\begin{array}{l}3.8 \\
3.8\end{array}$ \\
\hline $\begin{array}{l}\text { Ahilan and } \\
\text { Sleath (1987) }\end{array}$ & OWT & Half & $\begin{array}{l}5 \\
4\end{array}$ & $\begin{array}{l}1.137 \\
1.44\end{array}$ & $\begin{array}{l}4.0 \\
4.3\end{array}$ & $\begin{array}{l}0 \\
0\end{array}$ & \begin{tabular}{|l|}
$0.3-0.5$ \\
$1.1-1.2$
\end{tabular} & $\begin{array}{l}3.6-3.7 \\
4.7-4.9\end{array}$ \\
\hline $\begin{array}{l}\text { Watanabe and } \\
\text { Isobe (1990) }\end{array}$ & OWT & Full & $\begin{array}{l}11 \\
65\end{array}$ & $\begin{array}{l}2.65 \\
2.65\end{array}$ & $\begin{array}{l}0.18,0.87 \\
0.18,0.87\end{array}$ & $\begin{array}{l}0 \\
-0.3-0.25\end{array}$ & $\begin{array}{l}0.27-0.43 \\
0.27-0.52\end{array}$ & $\begin{array}{l}3.0,6.0 \\
3,6\end{array}$ \\
\hline King (1991) & OWT & Half & 178 & 2.65 & $0.1-1.1$ & 0 & $0.3-1.2$ & $2.0-12.0$ \\
\hline $\begin{array}{l}\text { Dibajnia and } \\
\text { Watanabe } \\
(1992)\end{array}$ & OWT & Full & $\begin{array}{l}25 \\
76\end{array}$ & $\begin{array}{l}2.65 \\
2.65\end{array}$ & $\begin{array}{l}0.20 \\
0.20\end{array}$ & $\begin{array}{l}0 \\
-0.26-0.22\end{array}$ & \begin{tabular}{|l|}
$0.6-1.0$ \\
$0.61-1.24$
\end{tabular} & $\begin{array}{l}1.0-4.0 \\
1-4\end{array}$ \\
\hline $\begin{array}{l}\text { Ribberink and } \\
\text { Chen (1993) }\end{array}$ & OWT & Full & 4 & 2.65 & 0.128 & 0 & $0.6-1.2$ & 6.5 \\
\hline $\begin{array}{l}\text { Ribberink and Al } \\
\text { Salem (1994) }\end{array}$ & OWT & Full & 10 & 2.65 & 0.21 & 0 & $0.7-1.4$ & $5.0-12.0$ \\
\hline $\begin{array}{l}\text { Delft Hydraulics } \\
\text { (1993-1999; see } \\
\text { Van Rijn et al. } \\
\text { 2001) }\end{array}$ & OWT & Full & 52 & 2.65 & $0.13-0.24$ & $-0.45-0.56$ & $0.37-1.49$ & 5,12 \\
\hline $\begin{array}{l}\text { Dohmen-Janssen } \\
\text { (1999) }\end{array}$ & OWT & Full & 27 & 2.65 & $0.13-0.32$ & $0.23-0.45$ & $0.46-1.85$ & $4-12$ \\
\hline $\begin{array}{l}\text { Dohmen-Janssen } \\
\text { and Hanes } \\
(2002)\end{array}$ & LWF & Full & 4 & 2.65 & 0.21 & $\begin{array}{l}-0.05-- \\
-0.03\end{array}$ & $0.88-1.5$ & $6.5,9.1$ \\
\hline $\begin{array}{l}\text { Ahmed and Sato } \\
(2003)\end{array}$ & OWT & Full & 15 & 2.65 & $0.21-0.74$ & 0 & $1.16-1.85$ & 3.0 \\
\hline
\end{tabular}


Figure 27 shows the calculated and measured bed-load transport for the studied experiments using values on the empirical coefficients of $a_{w}=6$ and $b_{w}=b=4.5$ (see Figure 26 for the calibration of $a_{w}$ ). This new fit shows that if the effect of the critical Shields parameter on the mean sediment transport rate over a wave period is similar to steady flow, the total net rate is a function of the Shields parameter to the power 1.5 with a smaller value on the coefficient $a$ than for steady current. Soulsby (1997) found similar results using the Meyer-Peter and Müller (1948) equation (Equations 77 and 86 include the coefficient values 8 and 5.1, respectively). This lower value is partly due to the time averaging. It may also contain contributions owing to a phase lag between instantaneous sediment concentration and the velocity at the bottom.

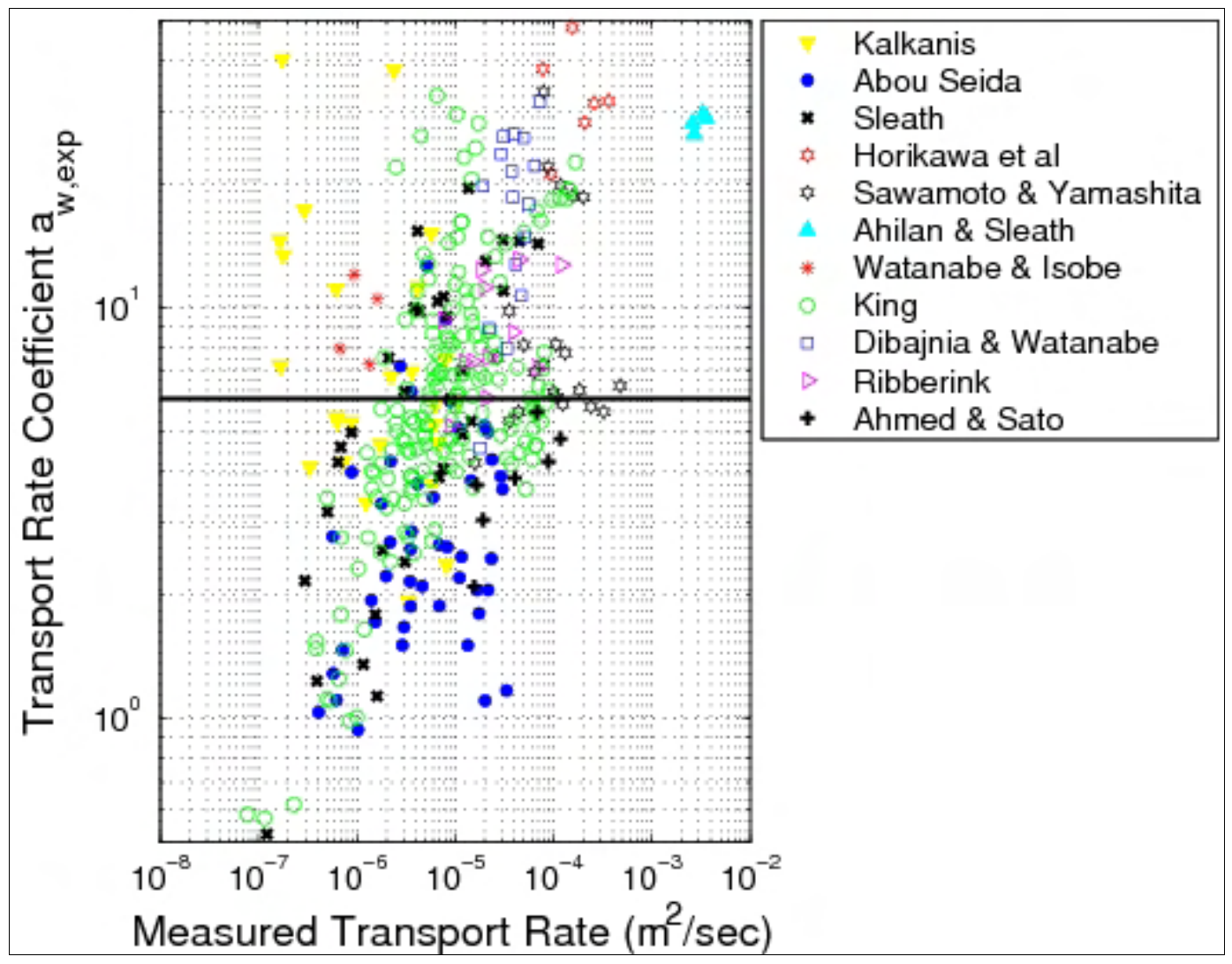

Figure 26. Calibration of coefficient $a_{w}$ using all experimental data with waves only (Nikuradse roughness calculated using Wilson (1989a) formula). 


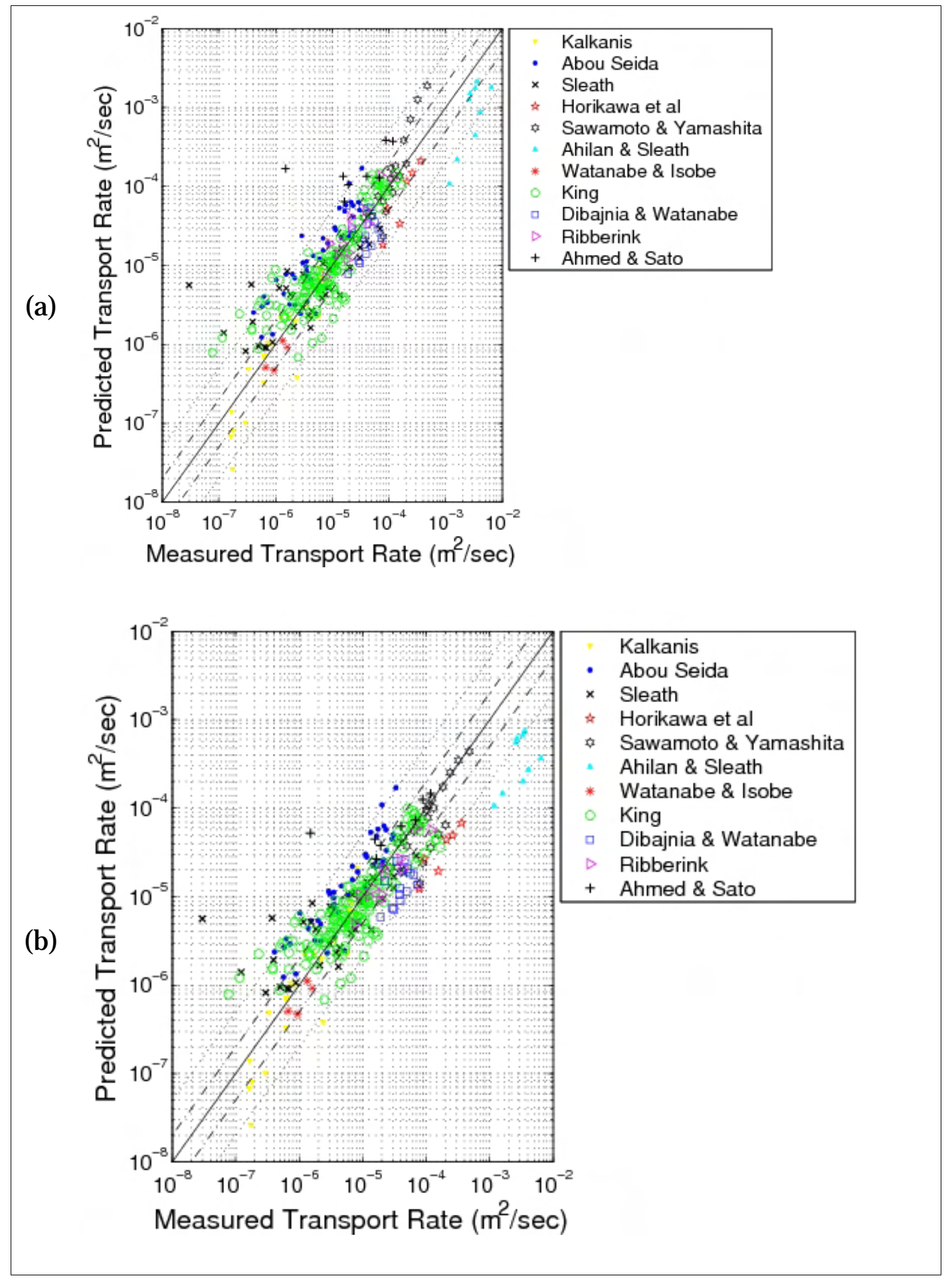

Figure 27. Comparison between bed-load transport predicted by Equation 90 and experimental data with waves (a) where $k_{s}$ is calculated using Wilson (1989a) formula, and (b) where $k_{s}=2 d_{50}$. 
In Figure 27(a), the predicted bed load using Equation 90 is plotted versus the measurements encompassing waves only. Even if some scatter occurs, the obtained results are seen to be good. Around 60 percent of the cases are predicted within a factor of 2 of the measured values. An additional difficulty in comparing the transport formula with the wave measurements is that no shear stress may be derived from the measurements, but the shear stress has to be calculated based on an estimate of the bed roughness. This introduces an extra element of uncertainty in the calculations of the sediment transport rates. The formulas proposed by Wilson (1966, 1989a, b) were employed to calculate the Nikuradse roughness:

$$
\begin{aligned}
& \frac{k_{s, s f c}}{d_{50}}=5 \theta_{c} \\
& \frac{k_{s, s f w}}{d_{50}}=5 \theta_{w}
\end{aligned}
$$

Both equations require an iterative method in solving for the Nikuradse roughness. An underestimation for the Ahilan and Sleath (1987) data set and an overestimation for the Sawamoto and Yamashita (1986) data set is observed. One explanation could be that large uncertainties are induced by this kind of iterative formula (Bayram et al. 2003; Camenen et al. 2006).

Using the Nikuradse skin roughness shows better agreement for the Sawamoto and Yamashita (1986) data set, but worse agreement for the Ahilan and Sleath (1987) data set (Figure 27(b)). Finally, it may be noted that Equation 90 slightly underestimates the sediment transport rate for the Kalkanis (1964) data set and overestimates the rate for the Abou-Seida (1965) and the Sleath (1978) data sets. Thus, it appears that the OT set-up does not yield the same behavior concerning the sediment transport as the OWT set-up.

Table 8 lists the percentages of predicted values within a factor of 2 deviation or a factor of 5 deviation and the root-mean-square error for the six studied formulas and all the data, as well as for the data encompassing complete wave cycles, using the Wilson (1989a) formula to compute the Nikuradse roughness $\left(k_{s}=k_{s, s f w}\right)$. The same calculations were also performed for all the data with the skin friction $\left(k_{s}=2 d_{50}\right)$ to compute the Shields parameter. 
Table 8. Predictive capability of bed-load transport rate within factor of 2 and 5 of measured values and root-mean-square errors, data from waves only.

\begin{tabular}{|l|l|l|l|l|l|l|l|l|l|}
\hline \multirow{2}{*}{ Author(s) } & \multicolumn{3}{|c|}{$\begin{array}{c}\text { All Data } \\
\left(k_{s}=k_{\mathrm{s}, \text { sfw }}\right.\end{array}$} & \multicolumn{3}{c|}{$\begin{array}{c}\text { Full Cycle Data } \\
\left(k_{\mathrm{s}}=k_{\mathrm{s}, \text { sfw }}\right.\end{array}$} & \multicolumn{3}{c|}{$\begin{array}{c}\text { All Data } \\
\left(k_{\mathrm{s}}=2 d_{50}\right)\end{array}$} \\
\cline { 2 - 12 } & Px2 (\%) & Px5 (\%) & $E_{\text {rms }}$ & Px2 (\%) & Px5 (\%) & $E_{\text {rms }}$ & Px2 (\%) & Px5 (\%) & $E_{\text {rms }}$ \\
\hline Madsen and Grant & 37 & 64 & 1.25 & 06 & 14 & 5.22 & 45 & 75 & 0.99 \\
\hline Bailard and Inman & 47 & 75 & 0.92 & 38 & 57 & 3.81 & 45 & 73 & 0.93 \\
\hline Dibajnia and Watanabe & 36 & 75 & 0.49 & 49 & 85 & 0.79 & 36 & 75 & 0.49 \\
\hline Soulsby (coef. 2.5) & 36 & 75 & 0.98 & 20 & 52 & 3.92 & 39 & 76 & 0.97 \\
\hline Ribberink & 29 & 73 & 1.08 & 20 & 46 & 4.14 & 36 & 76 & 1.03 \\
\hline Equation 90 & 59 & 86 & 0.77 & 34 & 58 & 3.84 & 57 & 86 & 0.78 \\
\hline
\end{tabular}

Equation 90 yields the best overall results. For the full cycle data, because the phase lag is not taken into account, the agreement is not as good, and the scatter is larger $\left(E_{r m s}=3.8\right)$. The Madsen and Grant (1976) formula tends to overestimate the sediment transport for large values of the shear stress. This formula is proportional to the Shields parameter to the power 3 and calibrated with lower shear stress data. Soulsby (1997) suggested to use the maximum shear stress in his formula, which causes a large overestimation of the transport rates. Replacement of a coefficient value of 2.5 instead 5.1 improves the results (results in Table 8), but it still indicates an overestimation of the sediment transport for low shear stresses. If the mean shear stress is used instead of the maximum shear stress (with the coefficient 5.1), results are better and similar to those given by Equation 90, except for data with low sediment transport rates, which are typically overestimated. The Bailard and Inman (1981), Dibajnia and Watanabe (1992), and Ribberink (1998) formulas also overestimate the weak sediment transport rate data. This means that the effect of the critical Shields parameter is not taken into account properly (not included in the Bailard and Inman (1981) and Dibajnia and Watanabe (1992) formulas).

The Ribberink (1998) formula typically overestimates the sediment transport rate. Using the coefficient values $(7.9,1.97)$ instead of $(11,1.65)$ or using $k_{s}=d 50$, as Ribberink (1998) proposed, improves the results. However, this is not in accordance with the formula originally proposed for a steady current (Equation 79) or with physical values of the Nikuradse roughness. The Dibajnia and Watanabe (1992) formula, because it takes 
the phase lag into account, allows the sediment transport to be in the opposite direction to the waves for fine sediment. Thus, it presents the overall best results for the full cycle data and a much lower scatter $\left(E_{r m s}<1\right)$. On the other hand, it yields poor results for the half-cycle data. Finally, it should be noted that results from the OT data are typically more scattered. This may be a result of the shear stresses being close to the critical shear stress, inducing additional randomness, as well as uncertainty in the measurements.

The main theoretical difference between the Ribberink (1998) relationship (Equation 87) and Equation 90 is that the former equation is a mean value of the instantaneous sediment transport over the wave period, whereas the latter calculates the sediment transport from the mean shear stress. Using the mean value over the wave period of the instantaneous sediment transport induces a sediment transport rate 20 percent larger than using the same equation with the mean shear stress over the period. Moreover, as was observed previously, phase-lag effects seem to be non-negligible, even for relatively small wave orbital velocities. This may explain the overestimation observed for the Ribberink formula.

Another remark to be made (Figure 28) is that the direction of the net sediment transport may be opposite to the direction of the waves. Ribberink and Chen (1993) and Dibajnia and Watanabe (1992) observed this phenomenon for highly asymmetric waves and fine sediment $\left(d_{50} \leq 0.2 \mathrm{~mm}\right)$. It is caused by the phase lag in the response of the sand to the fluid motion. The quantity of sediment in suspension depends not only on the instantaneous velocity, but also on the settling velocity. In the case of oscillatory flow, not all the sand grains put into suspension during the first half-period settle during the same half-period. The proportion still in suspension is then carried away in the opposite direction during the second half-period. Dohmen-J anssen (1999) and Dohmen-J anssen and Hanes (2002) introduced a parameter to describe the phase lag.

$$
p_{p l}=\frac{2 \pi \delta_{s}}{W_{s} T_{w}}
$$

where $\delta_{s}$ is the thickness of the sheet-flow layer. Following Asano (1992) and Dohmen-J anssen and Hanes (2002), $\delta_{s}=10 d_{50} \theta_{w}$. 


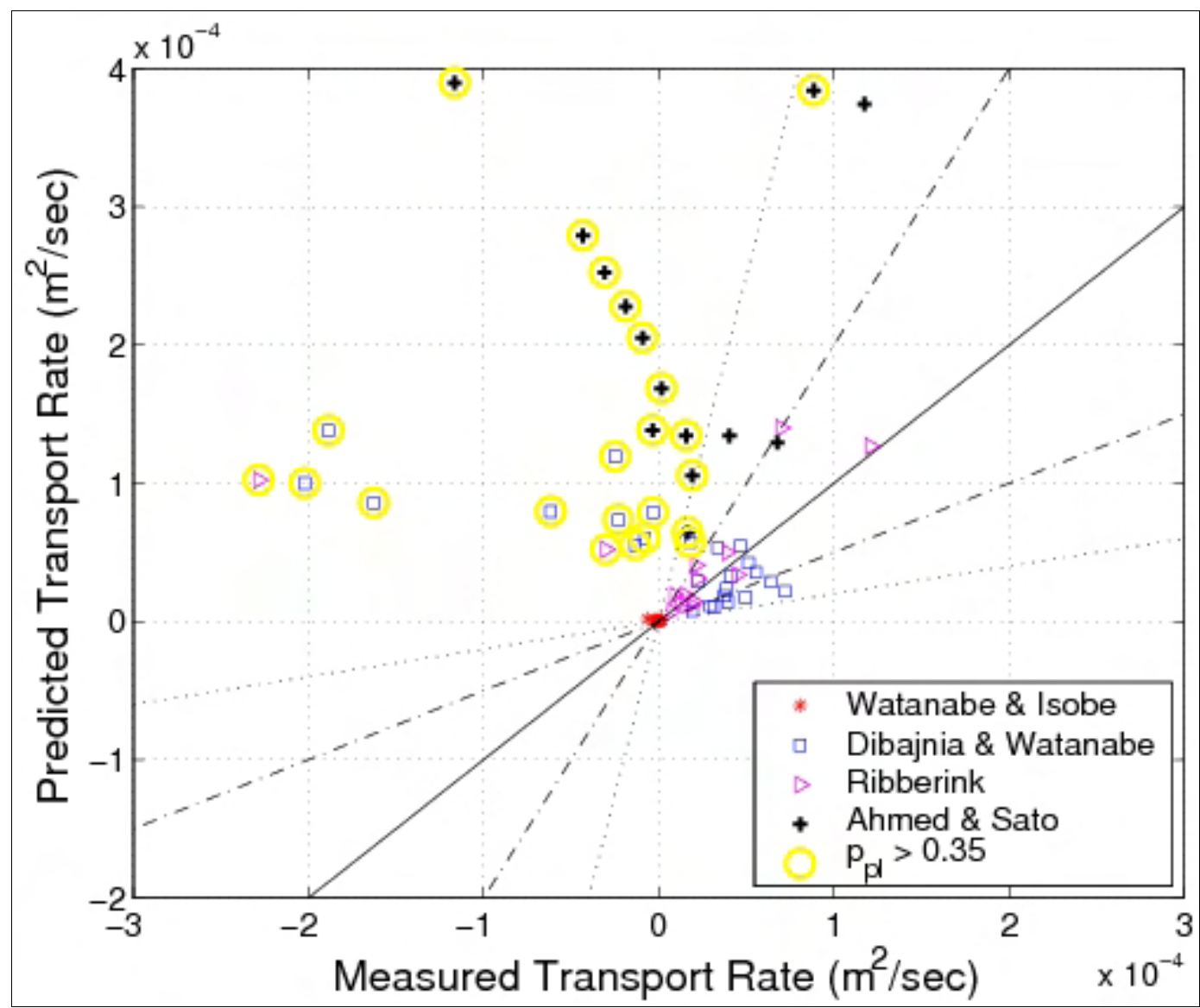

Figure 28. Comparison between bed-load transport predicted by Equation 90 and experimental data over full wave cycle ( $k_{s}$ calculated using Wilson (1989a) formula, $k_{s}=k_{s, s f w}$ ).

They observed that phase-lag effects occur if $p_{p l}>0.3-0.4$. Figure 28 plots the cases where strong phase lag occurs (circles). These data points correspond to an overestimation (and/ or wrong direction) of the predicted values using Equation 90. Thus, it appears that the proposed formula is mainly restricted to cases without significant phase lag. This phenomenon is not included in Equation 90, but could be approximated by adding a correction factor (Dohmen-J anssen 1999; Dohmen-J anssen and Hanes 2002; Camenen and Larroudé 2003).

\section{Bed-load transport by waves and currents}

Stirring by waves in the presence of a steady current tends to increase the total sediment transport significantly. Also, as shown in the previous section, asymmetric waves can transport sediment. Thus, to generalize the proposed formula to encompass both waves and current, a modification of the formula is proposed including the total shear stress obtained from the interaction between waves and current. 


\section{Development of new formula}

The conceptual model proposed for the case of waves only (Equation 90) can be extended to the interaction between waves and current. Assuming that $U_{s}$ is proportional to the shear velocity at the bottom, a dependence on $\left|\theta_{c w \text {,onshore }}+\theta_{c w \text {,offshore }}\right|^{1 / 2}$ may be assumed for $U_{s}$, where the interaction between waves and current is taken into account. The representative shear

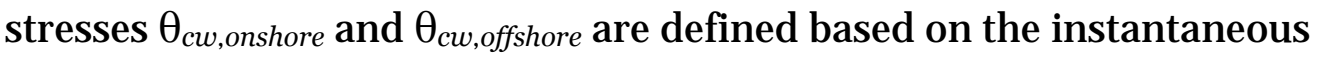
Shields parameter in the direction of the wave for positive and negative values of $\theta_{c w}(t)$, respectively (Figure 29). For an arbitrary angle $\varphi$ between the waves and the current, this yields the same equations as Equation 88, where $\theta_{w}$ is replaced by $\theta_{c w}$, and $T_{w c}$ and $T_{w c}$ are the half-periods where the instantaneous velocity $u(t)=U_{c} \cos \varphi+u_{w}(t)$ (or instantaneous Shields parameter) is onshore $(u(t)>0)$ or offshore $(u(t)<0)$, respectively (Figure 29). The representative shear stresses $\theta_{c w, \text { onshore }}$ and $\theta_{c w, o f f s h o r e}$ are defined as quadratic values of the instantaneous Shields parameter in the direction of the wave for positive and negative values of $\theta_{c w}(t)$, respectively (Figure 29). For an arbitrary angle $\varphi$ between the waves and the current, this yields:

$$
\begin{aligned}
& \theta_{c w, \text { onshore }}=\frac{1}{T_{w c}} \int_{0}^{T_{w c}} \theta_{c w}(t) d t \\
& \theta_{c w, \text { offshore }}=\frac{1}{T_{w t}} \int_{T_{w c}}^{T_{w}} \theta_{c w}(t) d t
\end{aligned}
$$

where $T_{w c}$ and $T_{w t}$ are the half-periods where the instantaneous velocity $u=U_{c} \cos \varphi+u_{w}(t)$ (or instantaneous Shields parameter) is onshore $(>0)$ or offshore $(<0)$, respectively. The instantaneous Shields parameter is defined as follows:

$$
\theta_{c w}(t)=\frac{\frac{1}{2} f_{c w}\left|U_{c} \cos \varphi+u_{w}(t)\right|\left(U_{c} \cos \varphi+u_{w}(t)\right)}{(s-1) g d_{50}}
$$




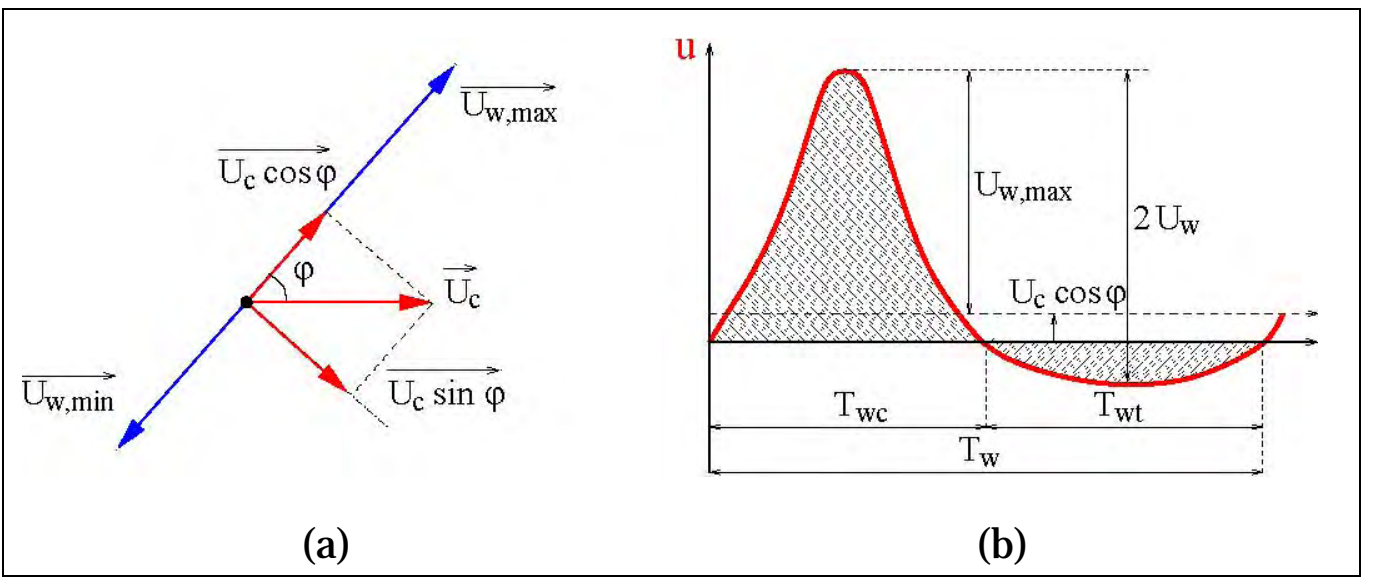

Figure 29. Definition sketches for (a) wave and current interaction, and (b) typical velocity variation over wave period in direction of wave propagation including effect of steady current.

where $f_{c w}$ is the friction coefficient for an interaction between wave and current. This coefficient is assumed to be constant over the wave period as a first approximation but, as already noted, a temporal variation exists. Madsen and Grant (1976) suggested that $f_{c w}$ could be obtained as a linear combination of the current-related $\left(f_{c}\right)$ and wave-related $\left(f_{w}\right)$ friction coefficients according to Equation 66.

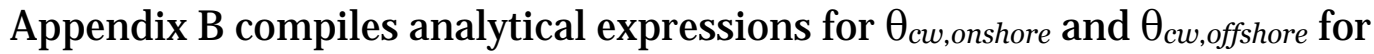
different wave theories or approximations.

The assumption that the moving sediment layer thickness $\Delta_{s}$ is proportional to the total mean bottom shear stress gives a dependence on $\theta_{c w, m}$ for the transport rate (the stirring at the bottom by the waves and current together is mobilizing the layer). For an arbitrary angle $\varphi$ between the waves and the current, the mean and maximum combined Shields parameters at the bottom $\theta_{c w, m}$ and $\theta_{c w}$, respectively, are written:

$$
\begin{aligned}
\theta_{c w, m} & =\left(\theta_{c}^{2}+\theta_{w, m}^{2}+2 \theta_{w, m} \theta_{c} \cos \varphi\right)^{1 / 2} \\
\theta_{c w} & =\left(\theta_{c}^{2}+\theta_{w}^{2}+2 \theta_{w} \theta_{c} \cos \varphi\right)^{1 / 2}
\end{aligned}
$$

where the magnitude of the mean (maximum) shear stresses from the waves and current were added (vector addition). A more sophisticated approach would include the time variation of the waves in this addition. 
In the derivation of a transport formula where waves contribute to the transporting velocity, it is convenient to determine the transport rate parallel and perpendicular to the direction of wave propagation. Then, for an arbitrary angle $\varphi$ between the waves and the current, Equation 81 can be written in the following manner.

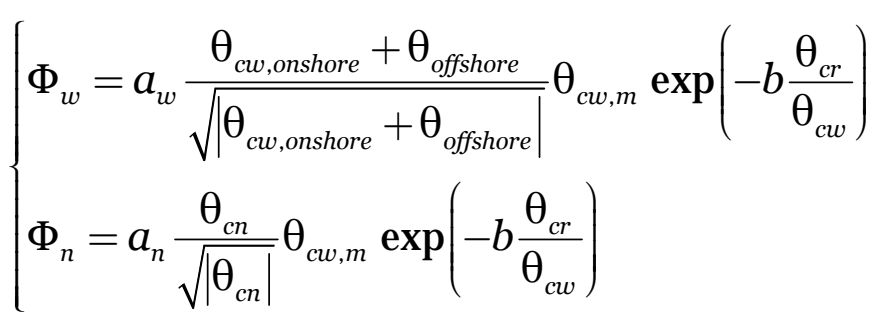

where $w$ and $n$ correspond, respectively, to the wave direction and the direction normal to the wave direction, $\theta_{c n}=\frac{1}{2} f_{c} U_{c}^{2} \sin \varphi|\sin \varphi| /\left((s-1) g d_{50}\right)$, and $a_{w}, a_{n}$, and $b$ are empirical coefficients as before. The same value $b=4.5$ is retained. For consistency with previous results obtained for current only and waves only, the following relationship is proposed for the coefficient $a_{w}$ :

$$
a_{w}=6+6 Y \text { with } Y=\frac{\theta_{c}}{\theta_{c}+\theta_{w}}
$$

Also, we take $a_{n}=12$.

The overall effect of the critical shear stress on the sediment transport over a wave period may be estimated using the same approximation as for the steady current. However, because Soulsby (1997, pp. 104-106) proposed to compute the critical shear stress based on the maximum shear stress, the maximum Shields parameter $\theta_{c w}$ is used.

For $\varphi=90$ deg and $\theta_{w} \gg \theta_{c}, \theta_{c w} \approx \theta_{w}$, that is, the waves are perpendicular to the current and control the stirring of the sediment. If $\theta_{c}=0$ (no current) with sinusoidal waves, $\theta_{c w}=\theta_{w}$ and $\theta_{c w \text {,onshore }}+\theta_{c w, \text { offshore }}=0$, which gives no bed load unless wave asymmetry is included. 


\section{Comparison with experimental data}

To investigate bed-load transport where waves and current are interacting, several existing data sets were compiled and analyzed (Table 7). Most of the data are from OWT experiments implying large orbital velocities with prevailing bed-load transport. More recently, Dohmen-J anssen and Hanes (2002) carried out experiments in a large-scale wave flume (LWF). For nonbreaking waves, they found that the bed-load sediment transport represents around 90 percent of the total load. These experiments showed that results are consistent with those observed in oscillating water tunnels (OWTs), although differences in the suspended sediment concentration and the total sediment transport rate are apparent. Net transport rates under waves were found to be about a factor 2.5 larger than in uniform horizontal oscillatory flows. They explained this by referring to the differences between boundary layer flows in OWT sand under free surface gravity waves. This difference may partly be attributed to the onshoredirected boundary layer streaming that is present under waves and is absent in horizontal oscillatory flow.

Figure 30(a) plots the bed-load transport predicted by Equation 99 against experimental data for current and waves interacting (with $U_{c}>0.02 \mathrm{~m} / \mathrm{sec}$ ). Good agreement is observed, and more than 50 percent of the data points are predicted within a factor of 2 of the measured values. A major part of the discrepancy $\left(E_{r m s} \approx 2\right.$, see Table 9 ) can be explained by the difficulty in predicting roughness values for oscillatory sheet flow (no measured values available) and because phase-lag effects are not included in the formula (some large overestimations or even incorrect transport directions are observed if strong phase lag occurs, as indicated in Figure 28). The sediment transport rates for the Delft Hydraulic data set are slightly overestimated if the Wilson formula is used and better predicted using the Nikuradse skin roughness (Figure 30(b)). On the other hand, the sediment transport rates for the Dibajnia and Watanabe (1992) data set are underestimated if the skin friction is used and better predicted if the Wilson formula is employed. The four data points from the large wave flume experiment are well predicted (within a factor of 2 of the measured values). 


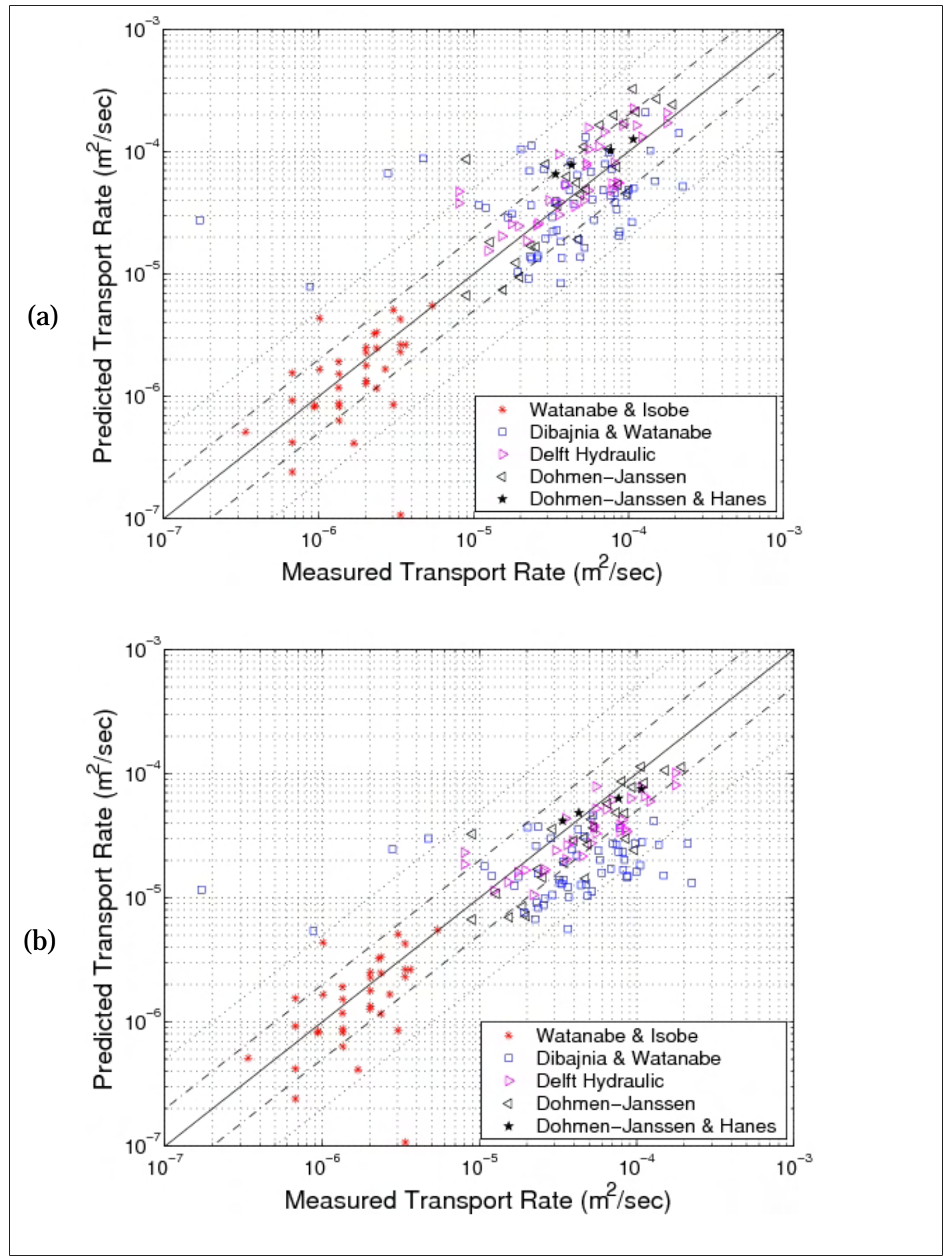

Figure 30. Comparison between bed-load transport predicted by Equation 99 and experimental data with current $\left(U_{c}>0.02 \mathrm{~m} / \mathrm{sec}\right)(\mathrm{a})$ where $k_{s}$ is calculated using Wilson (1989a) formula, and (b) where $k_{s}=2 d_{50}$. 
Table 9. Prediction of bed-load transport rate within factor of 2 and 5 of measured values and root-mean-square errors using data on waves and current combined $\left(\left|U_{c}\right|>0.02 \mathrm{~m} / \mathrm{sec}\right)$.

\begin{tabular}{|l|l|l|l|l|l|l|}
\hline \multirow{2}{*}{ Author(s) } & \multicolumn{3}{|c|}{$\begin{array}{c}\text { All Data } \\
\left(k_{s}=k_{s, s f w}\right.\end{array}$} & \multicolumn{3}{c|}{$\begin{array}{c}\text { All Data } \\
\left(k_{s}=2 d_{50}\right)\end{array}$} \\
\cline { 2 - 8 } & Px2 (\%) & Px5 (\%) & $E_{\text {rms }}$ & Px2 (\%) & Px5 (\%) & $E_{\text {rms }}$ \\
\hline Bailard and Inman & 47 & 70 & 2.07 & 41 & 68 & 2.10 \\
\hline Dibajnia and Watanabe & 41 & 72 & 1.74 & 41 & 72 & 1.74 \\
\hline Ribberink & 18 & 44 & 4.13 & 32 & 52 & 4.01 \\
\hline Equation 99 & 54 & 77 & 2.03 & 46 & 74 & 2.05 \\
\hline
\end{tabular}

It seems that the differences observed by Dohmen-J anssen and Hanes (2002) between the LWF and OWT experiments for the linear relationship between the net transport rates and the third-power velocity moment are reduced by inclusion of the friction coefficient (within the Shields parameter). Finally, contrary to comparisons with other formulas, good agreement is observed for the Watanabe and Isobe (1990) data set, corresponding to small wave orbital velocities and periods. This means that the effect of the critical Shields parameter is well described. It should be noted that for this data set, ripples were sometimes observed, which induced stronger suspension and phase-lag effects. Appearance of ripples may explain the opposite direction of the sediment transport observed for some of the data points as pointed out by Dibajnia and Watanabe (1992).

\section{Comparison with existing formulas for waves and current}

Only the Bailard and Inman (1981), Dibajnia and Watanabe (1992), and Ribberink (1998) formulas include the effects of a current. Table 9 lists the percentage of predicted values, within a factor of 2 or a factor of 5 deviation from the measurements, for the four studied formulas presented for all the data where a mean current is present using the Wilson relationship $\left(k_{s}=k_{s, s f w}\right)$ or the skin friction $\left(k_{s}=2 d_{50}\right)$ to compute the Nikuradse roughness.

As seen in Table 9, a larger discrepancy exists compared to the steady current data, because the total shear stress has to be estimated instead of using measured values. Equation 99 still yields the best overall results. The Bailard and Inman (1981) formula overestimates the sediment transport rate for low shear stresses (e.g., the Watanabe and Isobe (1990) data set), and underestimates the rate for the Dibajnia and Watanabe (1992) data set 
and for all the cases where strong phase lag occurs. Nevertheless, it gives good overall agreement with the data. As already observed, the Ribberink (1998) formula overestimates the transport rates when waves are present. Results are improved using the Nikuradse skin roughness, but they remain poor compared to the other predictive formulas. Finally, although the Dibajnia and Watanabe (1992) formula shows good overall results, it overestimates the data from Delft Hydraulics and seems not to be adequately sensitive to the bottom shear stress.

\section{Phase-lag effects on sediment transport in sheet flow}

\section{Introduction}

Manohar (1955) performed one of the first studies on the inception of sheet flow (disappearance of the wave ripples) by using an oscillatory tray facility. In more recent times, sheet flow processes have typically been studied in oscillating water tunnels because field measurements close to the bed are not possible during storms, when sheet flow should prevail (e.g., Horikawa et al. 1982; Sawamoto and Yamashita 1986; King 1991; Asano 1992; Dibajnia and Watanabe 1992; Ribberink and Chen 1993; Ribberink and Al-Salem 1994; Li and Sawamoto 1995; Zala Flores and Sleath 1998; Dohmen-J anssen 1999; and Dohmen-J anssen et al. 2002). These studies considerably improved knowledge on the thickness of the sheet-flow layer, the time-dependent concentration profile inside the sheet-flow layer, and the resulting sediment transport rates.

Ribberink (1998) showed that a quasi-steady formula based on the MeyerPeter and Müller (1948) concept successfully describes the net sediment transport rates with the exception of relatively fine sands $\left(d_{50}<0.2 \mathrm{~mm}\right.$ ) and short wave periods ( $T_{w}<3 \mathrm{sec}$ ). Dibajnia and Watanabe (1992) carried out experiments with short period waves and found that in many cases a quasi-steady transport model failed to describe the magnitude and the direction of the net transport rate. They hypothesized that the phase lag between velocity and concentration was responsible for this discrepancy. Similar observations were made by Ribberink and Chen (1993) with fine sediment $\left(d_{50}=0.13 \mathrm{~mm}\right.$ ) and large asymmetric waves. DohmenJ anssen et al. (2002) extended the data set on net transport rates and time-dependent velocities and concentrations under sheet-flow conditions during sinusoidal oscillatory flow combined with a net current for sand with different grain sizes (i.e., $d_{50}=0.13,0.21$, and $0.32 \mathrm{~mm}$ ). They suggested that this limitation of quasi-steady models may be due to a 
phase lag, such as delayed entrainment and settling of sand grains, and proposed a simple model to take these effects into account. The lag between the sediment concentration and the flow was characterized by the ratio between the fall time of the sediment particle (which may be represented by the ratio between the sheet-flow layer thickness and the settling velocity) and the wave period.

Although knowledge on phase lag in sheet flow has improved recently, only a few models allow for a quantitative description of this phenomenon. The formula proposed by Dibajnia and Watanabe (1992) appears to be the only one that shows a correct behavior in the sheet-flow regime (Camenen and Larroudé 2003). More recently, Dohmen-J anssen et al. (2002) proposed a correction of the sediment transport (based on the Ribberink (1998) formula if a phase lag occurs that allows for improved estimates of the net bed-load transport rate.

Because sheet flow sediment transport occurs near the bed, it is often assumed that the response time of the sediment is much shorter than the wave period. However, in practice, a certain delay exists for the sand to respond to the fluid. Thus, the quantity of sediment in suspension is primarily related to the instantaneous velocity, but it also depends on the settling velocity. In oscillating flow, not all sand grains put into suspension during the first half-period are transported and settle during this period. The proportion still in suspension is then carried away in the opposite direction during the second half-period.

\section{A simple conceptual model}

A simple conceptual model was introduced by Dohmen-J anssen (1999) to include the phase lag in sediment transport: the instantaneous bed-load transport is assumed to be proportional to the instantaneous sediment concentration at the bottom (taken constant over the sheet-flow layer) multiplied by the instantaneous horizontal velocity at the bottom. Assuming that the instantaneous sediment concentration is a function of the instantaneous velocity to the power 2 , but with a possible phase lag $\phi_{s}$, the effect of this phase lag on the sediment transport may be estimated. For a sinusoidal wave, the sediment transport reduction due to a phase lag may be expressed as follows: 


$$
\begin{aligned}
r_{p l 1} & =\frac{q_{s, n e t}}{q_{s, n e t, \phi_{s}=0}}=\frac{\int_{0}^{T_{w}}\left(r_{u}+\cos \omega t\right)\left[r_{u}+\cos \left(\omega t+\phi_{s}\right)\right]^{2} d t}{\int_{0}^{T_{w}}\left(r_{u}+\cos \omega t\right)^{3} d t} \\
& =\frac{r_{u}^{2}+1 / 2+X}{r_{u}^{2}+3 / 2}
\end{aligned}
$$

where $r_{u}=U_{c} / U_{w}$, in which $U_{c}$ is the mean current averaged over the depth, and $X=\cos \phi_{s}$.

An analytical solution also exists for a second-order wave assuming $u_{w}\left(\cos \omega t+r_{w} 2 \omega t\right)$ (see also Figure 33):

$$
\begin{aligned}
r_{p l 2} & =\frac{\int_{0}^{T_{w}}\left(r_{u}+\cos \omega t+r_{w} \cos 2 \omega t\right)\left[r_{u}+\cos \left(\omega t+\phi_{s}\right)+r_{w} \cos 2\left(\omega t+\phi_{s}\right)\right]^{2} d t}{\int_{0}^{T_{w}}\left(r_{u}+\cos \omega t+r_{w} \cos 2 \omega t\right)^{3} d t} \\
& =\frac{r_{u}^{3}+r_{u}\left[1 / 2+X+r_{w}^{2}\left(2 X^{2}-1 / 2\right)\right]+r_{w}\left(X^{2}+X-1 / 2\right) / 2}{r_{u}^{3}+3 / 2 r_{u}\left(1+r_{w}^{2}\right)+3 / 4 r_{w}}
\end{aligned}
$$

Equations 101 and 102 are functions only of the wave profile (i.e., of the coefficients $r_{u}$ and $r_{w}$ ) and the phase lag of the sediment suspension $\phi_{s}$. Figure 31 shows the effect of the coefficients $r_{u}$ and $\phi_{s}$ in Equation $101\left(r_{w}\right.$ $=0$ ) displayed. As observed by Ribberink and Chen (1993) and Ahmed and Sato (2003), sediment transport in the direction opposite to the waves is possible for large values on $\phi_{\mathrm{s}}$ and if $r_{u}<\sqrt{2} / 2$ (sinusoidal waves) or $r_{u}^{3}+r_{u}\left(3 r_{w}^{2}-1\right) / 2-1 / 4 r_{w}<0$ (second-order wave).

It should be noted that Equation 102 does not have a solution if the denominator Den $=r_{u}^{3}+3 / 2 r\left(1+r_{w}^{2}\right)+3 / 4 r_{w}=0$ (the net sediment transport rate equals zero if no phase lag is assumed). The asymmetry factor $r_{w}$ modifies the results significantly, particularly if Den is close to zero where the function diverges (Figure 32). If Equation 101 yields a minimum value for $r_{p l}$ equal to -0.35, Equation 102 produces much smaller values on $r_{p l}$ if the mean current is relatively weak $\left(\min \left(r_{p l}\right) \approx-0.5\right.$ when $\left.r_{u}=0\right)$ and opposite to the waves $\left(r_{p l} \approx-1.1\right.$, if $r_{u}=0.05, r_{w}=0.20$ and $X=\cos \phi_{s} \approx \pi / 2$ ). Furthermore, Equation 102 is not a symmetric function of $r_{u}$ and $r_{p l}$, and it may be greater than 1 if Den $<0$ and close to zero (Figure 32). 


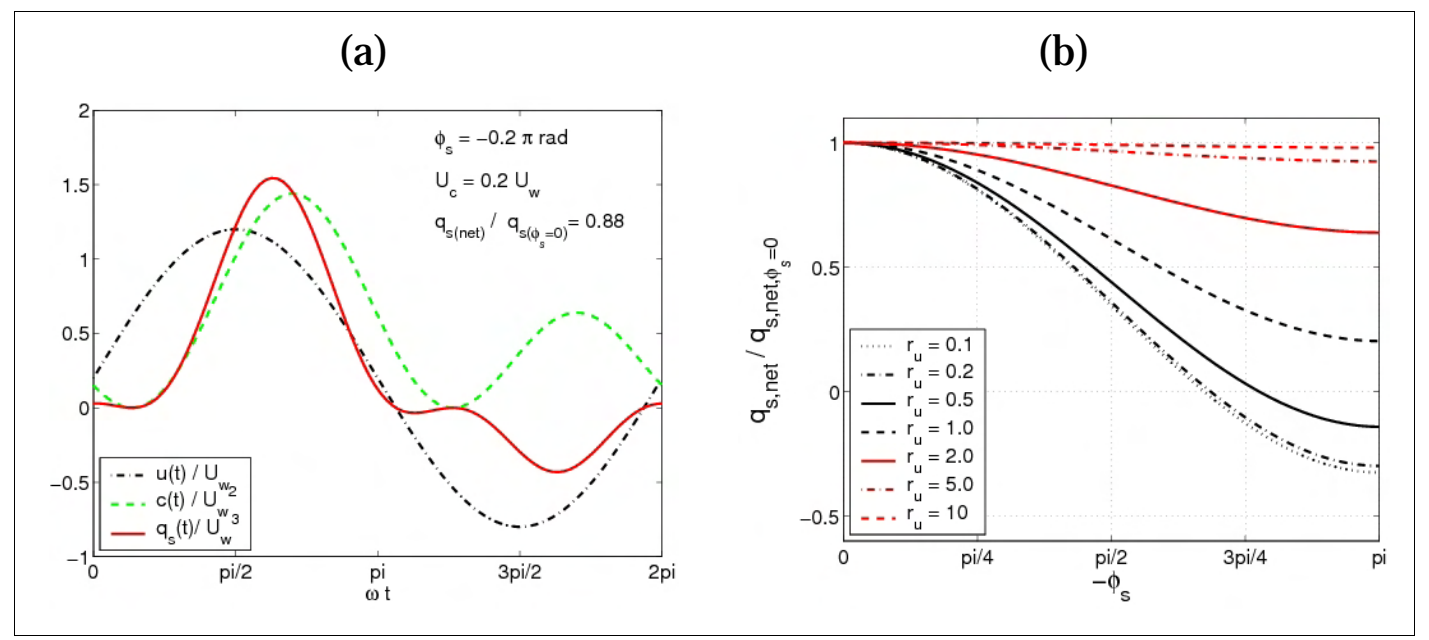

Figure 31. Phase-lag effect on sediment transport for sinusoidal wave with superimposed current when phase lag $\phi_{s}$ is introduced for concentration at bottom: (a) instantaneous variation in velocity, concentration, and bed-load rate for $U_{c} / U_{w}=0.2$ and $\phi_{s}=-0.2 \pi$, and (b) effect on sediment transport for varying $\phi_{s}$ and ratio $U_{c} / U_{w}$.

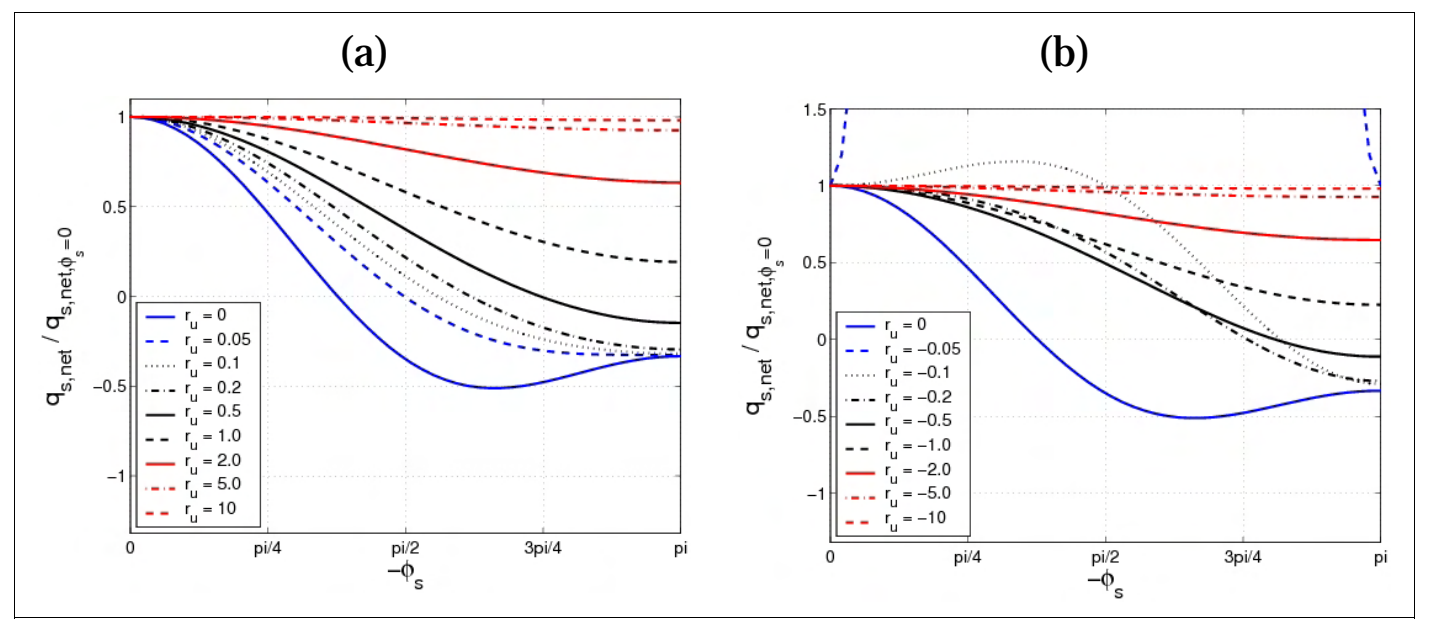

Figure 32. Phase-lag effects on sediment transport for second-order Stokes wave with (a) positive, or (b) negative, and adding current introducing phase lag $\phi_{s}$ for concentration at bottom and with $r_{w}=0.2$.

Dohmen-J anssen (1999) and Dohmen-J anssen et al. (2002) proposed a more complex model where the sediment concentration varies over the sheet-flow layer following an exponential law. The solution obtained is similar to Equation 101 (or Equation 102, if a $2^{\text {nd }}$ order Stokes wave is used) in which $X=\cos \phi_{s}$ is replaced by a function of the phase-lag parameter $p_{p l}$ defined as in Equation 93. However, the model does not allow for small values on $r_{p l}$ (nor negative values) for large phase lag (the minimum value of $r_{p l}$ is 0.35 for a sinusoidal wave, and it is 0.0 for a second-order Stokes wave), but may indicate a possible relationship between $\phi_{s}$ and $p_{p l}$. 


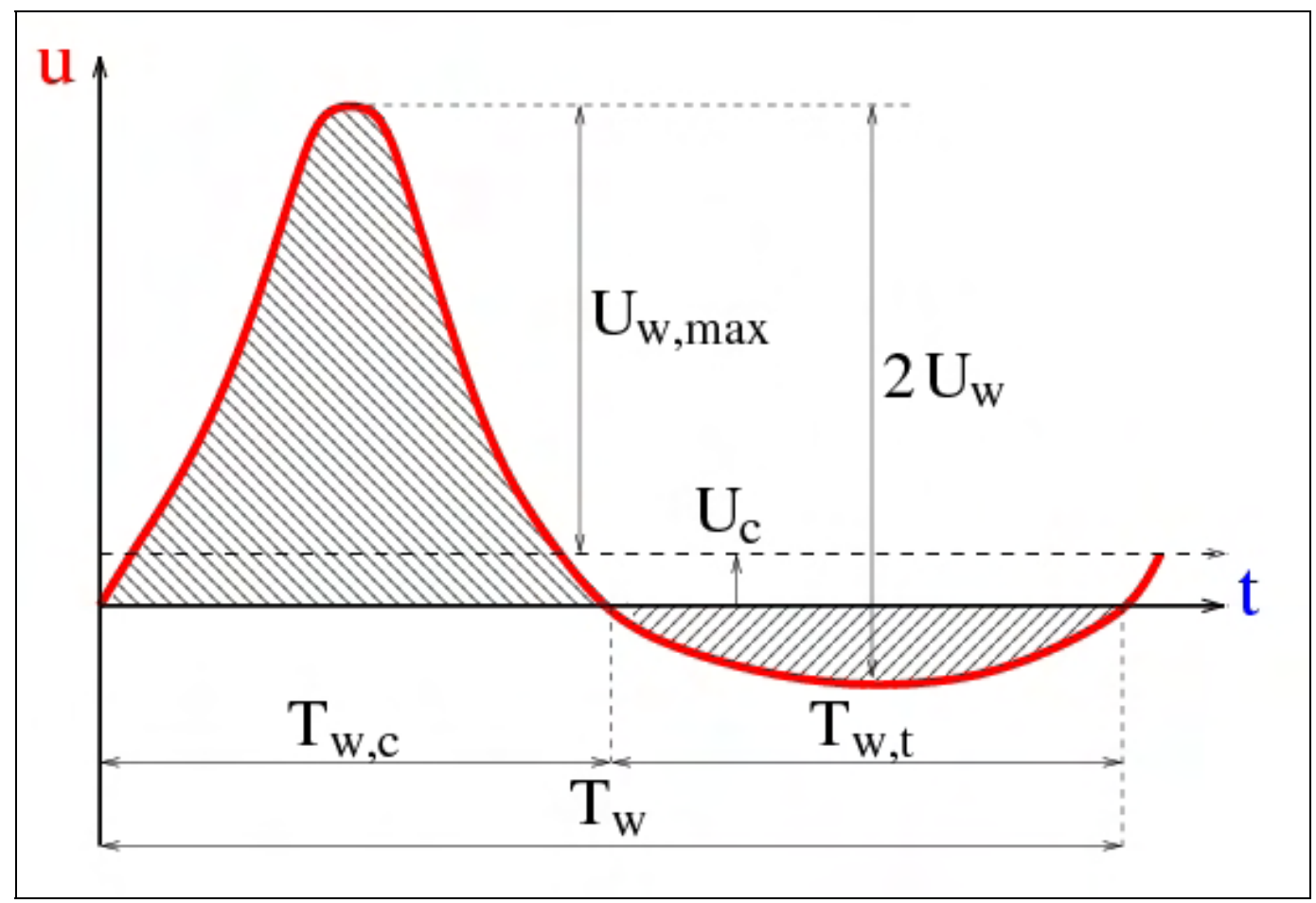

Figure 33. Notation for colinear wave and current interaction.

\section{Dibajnia and Watanabe formula}

Dibajnia and Watanabe (1992) proposed a sediment transport model that accounts for the phase lag. They considered the amount of sand that is entrained during a positive half-wave cycle. They determined the part that will be transported directly by the positive velocity during the first half cycle and the part that will still be in suspension as the flow reverses and therefore transported by the negative velocity during the following half cycle (see Figure 33). The same concept was proposed for the negative half cycle.

Dibajnia and Watanabe (1992) assumed that phase-lag effects occur as soon as sheet flow appears. Thus, following the criterion for the inception of sheet flow developed by Dibajnia (1991, Equation 55), they proposed to use the parameter $\omega_{p l}$ (Equation 54) for each half period:

$$
\omega_{p l, j}=\frac{\frac{1}{2} U_{w j}^{2}}{(s-1) g W_{s} T_{w j}}
$$


where $U_{w j}$ is the representative velocity for the positive or negative half period $T_{w j}$, and the subscript $j$ should be replaced either by onshore (direction of the wave) or offshore (opposite direction to the wave). Dibajnia and Watanabe (1992) assigned the indices $c$ for crest and $t$ for trough, instead of onshore and offshore, respectively. According to their data, sheet flow occurs as soon as $\omega_{p l, j}>1$. If no phase lag occurs, their formula is proportional to the mobility parameter for both half periods $\Psi_{j}$ (where $U_{w}$ is replaced by $U_{w j}$ ) as $\Omega_{j}=W_{s} T_{j} / d_{50}$ and $\Omega_{j}^{\prime}=0$. For the case where sheet flow is reached during both half periods, the phase-lag effects may thus be quantified as follows:

$$
r_{p l, D W}=\left[\frac{T_{w c} U_{w c}\left(\Omega_{c}^{3}+\Omega_{t}^{\prime 3}\right)-T_{w t} U_{w t}\left(\Omega_{t}^{3}+\Omega_{c}^{\prime 3}\right)}{T_{w c} U_{w c} \Psi_{c}^{3}-Y_{w t} U_{w t} \Psi_{t}^{3}}\right]^{0.55}
$$

where subscript $c$ indicates crest and $t$ trough, and:

$$
\Omega_{J}=\max \left(\omega_{p l, j}, 1\right) \frac{W_{s} T_{j}}{d_{50}} \text { and } \Omega_{j}^{\prime}=\left(\omega_{p l, j}-1\right) \frac{W_{s} T_{j}}{d_{50}}
$$

Dibajnia and Watanabe (1992) slightly modified their formula to take into account the phase lag occurring in the ripple regime by introducing the parameter $\omega_{c r}$. Thus, phase lag occurs if $\omega_{p l, j}>\omega_{c r}$, where $\omega_{c r}=1$ for the sheet-flow regime, and $\omega_{c r}=0.03$ for the ripple regime.

\section{Modification of Camenen and Larson formula for phase lag}

Following Dibajnia and Watanabe (1992), a modification of the Camenen and Larson (2005a, b) formula is proposed to take the phase lag into account. This formula is based on the "bed-shear stress concept" (function of the Shields parameter) and allows sediment transport in the direction of the wave using characteristic values on the Shields parameter for both half

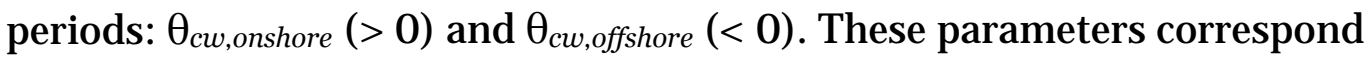
to the mean value of the instantaneous Shields parameter $\theta_{c w}(t)=1 / 2 f_{c w}\left(U_{c} \cos \varphi+u_{w}(t)\right)\left|U_{c} \cos \varphi+u_{w}(t)\right| /\left((s-1) g d_{50}\right)$ over each half period where $\varphi$ is the angle between the wave and current directions, and $f_{c w}$ is obtained using the Madsen and Grant (1976) relationship (Equation 96). Thus, the net sediment transport depends on the factor $\theta_{c w, n e t}=\theta_{c w, \text { onshore }}+\theta_{c w, \text { offshore. }}$ In the wave direction, the bed-load sediment transport may be expressed as follows (compare with Equation 99): 


$$
\Phi_{w}=a_{w} \frac{\theta_{c w, n e t}}{\sqrt{\left|\theta_{c w, n e t}\right|}} \theta_{c w, m} \exp \left(-b \frac{\theta_{c r}}{\theta_{c w}}\right)
$$

where $a_{w}$ and $b$ are coefficients, and $\theta_{c w, m}, \theta_{c w}$, and $\theta_{c r}$ are the mean values of the absolute instantaneous Shields parameter, the maximum Shields parameter, and the critical Shields parameter for the inception of motion, respectively.

The sediment phase lag is introduced in the formula by assuming that values of the Shields parameter for both half periods are modified due to this effect. A decrease in $\theta_{c w \text {,onshore }}$ and an increase in $\theta_{c w}$,offshore appear if the critical velocity for inception of sheet flow is reached:

$$
\theta_{c w, \text { net }}=\left(1-\alpha_{p l, b}\right) \theta_{c w, \text { onshore }}+\left(1+\alpha_{p l, b}\right) \theta_{c w, \text { offshore }}
$$

in which $\alpha_{p l, b}=\alpha_{\text {onshore }}-\alpha_{\text {offshore }}$ and:

$$
\alpha_{j}=\frac{v^{0.25} U_{w j}^{0.5}}{W_{s} T_{j}^{0.75}} \exp \left[-\left(\frac{U_{w, c r, s f}}{U_{c w j}}\right)^{2}\right]
$$

where $U_{c w j}$ is the root-mean-square value of the velocity (waves and current combined) over the half period $T_{w j}$, and the subscript $j$ should be replaced either by onshore or offshore. The effect of the main parameters (i.e., $U_{c w j}, T_{j}$, and $W_{s}$ ) was examined, and they were calibrated based on the compiled data set. The exponential function, describing the effect of the critical velocity on the inception of sheet flow, is proposed to allow a possible error in the estimation of $U_{w, c r, s f}\left(U_{w, c r, s f}\right.$ is calculated following Equation 57), i.e., $\alpha_{j} \neq 0$ even if $U_{c w j}$ is slightly lower than $U_{w, c r, s f}$. Strictly, $U_{w, c r, s f}$ should be compared to a maximum value of the velocity and not to a quadratic mean value. However, the results would only be modified by a constant factor in the exponential function, changing the fit of the empirical function $\alpha_{j}$. Thus, the coefficient quantifying the phase lag may be written as follows:

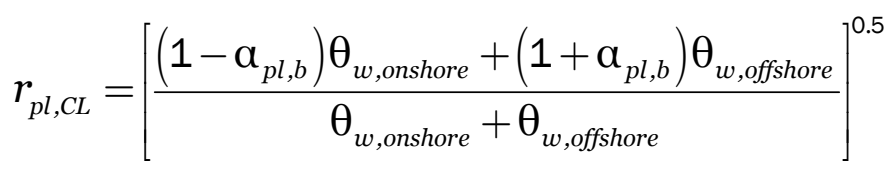




\section{Experimental data}

To investigate phase-lag effects on bed-load transport under waves and current, data sets from OWT experiments covering a wide range in values were compiled and analyzed. This kind of experiment has several advantages for the present study: large orbital velocities can be reached, bedload transport prevails, and a strong phase lag is often observed. Table 10 summarizes the data sets employed, where the type of experiment, sediment characteristics, and wave properties are listed.

Table 10. Summary of data on bed-load sediment transport in full-cycle oscillatory flow.

\begin{tabular}{|l|l|l|l|l|l|l|}
\hline Author(s) & Number & $\mathbf{s}$ & $\begin{array}{l}d_{50} \\
(\mathrm{~mm})\end{array}$ & $\begin{array}{l}U_{c} \\
(\mathrm{~m} / \mathrm{sec})\end{array}$ & $\begin{array}{l}U_{w, m a x} \\
(\mathrm{~m} / \mathrm{sec})\end{array}$ & $\begin{array}{l}T_{w} \\
(\mathrm{sec})\end{array}$ \\
\hline $\begin{array}{l}\text { Watanabe and Isobe } \\
(1990)\end{array}$ & 65 & 2.65 & $0.18,0.87$ & $-0.3-0.25$ & $0.27-0.52$ & $3.0,6.0$ \\
\hline $\begin{array}{l}\text { Dibajnia and Watanabe } \\
(1992)\end{array}$ & 76 & 2.65 & 0.20 & $-0.26-0.22$ & $0.61-1.24$ & $1.0-4.0$ \\
\hline Ribberink and Chen (1993) & 4 & 2.65 & 0.128 & $<0.05$ & $0.6-1.2$ & 6.5 \\
\hline $\begin{array}{l}\text { Ribberink and Al Salem } \\
(1994)\end{array}$ & 30 & 2.65 & 0.21 & $-0.11-0.56$ & $0.37-1.37$ & $5.0-12.0$ \\
\hline $\begin{array}{l}\text { Delft Hydraulics (1994- } \\
\text { 1999; see Van Rijn et al. } \\
\text { 2001) }\end{array}$ & 22 & 2.65 & $0.13-0.24$ & $-0.45-0.47$ & $0.46-1.49$ & $4.0-12.0$ \\
\hline Dohmen-Janssen (1999) & 27 & 2.65 & $0.13-0.32$ & $0.23-0.45$ & $0.46-1.85$ & $4.0-12.0$ \\
\hline Ahmed and Sato (2003) & 15 & 2.65 & $0.21-0.74$ & - & $1.16-1.85$ & 3.0 \\
\hline
\end{tabular}

\section{Calibration of conceptual model}

Calibration of the conceptual model is not as easy as the modification of the Camenen and Larson (2005a, b) formula. Indeed, it is difficult to estimate what the sediment transport rate would have been without phase-lag effects. Three methods are proposed to estimate $r_{p l}$ following Camenen and Larroudé (2003) by using a sensitivity analysis of the different parameters. Thus, as soon as no phase lag occurs, bed load might be:

- Independent of median grain size.

- Independent of wave period.

- Proportional to velocity moment to the power 3. 
Using experimental data where all the parameters are fixed except the median grain size (or the wave period), it is possible to estimate $r_{p l}$ assuming $r_{p l}=1$ for larger values of $d_{50}$ (or $T_{w}$ ), i.e., $q_{s, n e t}=q_{s, \text { net, } \phi_{s}}=0$. For the third method, the relationship between the bed-load rate and the velocity moment to the power 3 is assumed exact, if $U_{w}$ is small. A curve proportional to $\left\langle u^{3}>\right.$ is fitted to the experimental value of $q_{s, n e t}$ where the measured value of $U_{w}$ is the smallest, and compared to the other experimental values of $q_{s, n e t}$ where $U_{w}$ is higher (and where phase-lag effects are expected).

In total, 85 data values were derived using these three methods. It should be noted that several values do not have a solution by Equation 102; the estimated value of $r_{p l}$ was found to be smaller than the minimum value obtained by the model $\left(\min \left(r_{p l}\right) \approx-0.5\right.$ when $\left.r=0\right)$. In these cases, the $\phi_{s}$-value corresponding to the minimum of $r_{p l}$ was used. This illustrates the limitations of the conceptual model. However, these errors may also be induced by some limitations in the experimental set-up. Indeed, for some data from the Dibajnia (1991) experiment, $r_{p l}$ reaches -5. As discussed previously, the piston generated additional vortices for the shortest wave periods that induced more suspension, and thus artificially increased the phase-lag effect.

It seems that $\phi_{s}$ is well described by the parameter $\omega_{p l}$ (Equation 54 ) introduced by Dibajnia (1991), using $U_{w}$ and $T_{w}$ instead of $U_{w j}$ and $T_{w j}$. The following empirical relationship is proposed (curve in Figure 34(a)):

$$
\phi_{s}=\pi \tanh \left(1.5 \omega_{p l}^{1.5}\right)
$$

Figure 34(a) plots the points obtained for $\phi_{s}$ from the three different methods against $\omega_{p l}$. Although significant scatter is observed, most of the data are correctly predicted by Equation 110 (solid line; the dashed lines correspond to Equation 110 with $\omega_{p l}^{\prime}=0.5 \omega_{p l}$ and $2 \omega_{p l}$ ). Figure 34(b) shows a comparison between the observed and predicted values on $r_{p l}$ from the experiment (using Equations 102 and 110). The factor $r_{p l}$ is generally well predicted; it should be remembered that large uncertainties are induced through the calculation of $r_{p l}$. Even if large underestimation occurs for extreme cases (from the wave period method) where $r_{p l}<-0.5$, the general tendency obtained by Equations 102 and 110 is encouraging. 


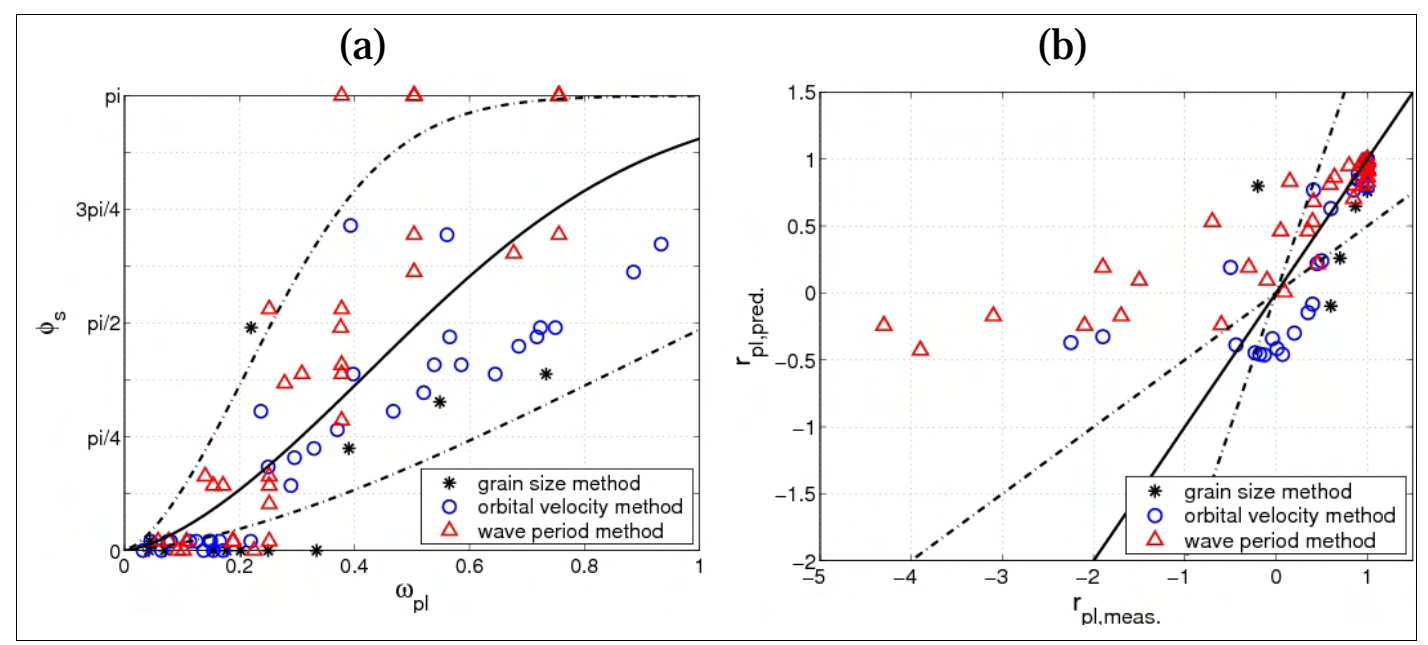

Figure 34. Calibration of conceptual model against data.

\section{Influence of median grain size}

Strong phase-lag effects were first observed for fine sand ( $d_{50}=0.2 \mathrm{~mm}$, Dibajnia 1991; $d_{50}=0.13 \mathrm{~mm}$, Ribberink and Chen 1993), where a net sediment transport opposite to the direction of the waves (and mean current) was observed. The specific study on grain-size influence on sediment transport in oscillatory sheet flow by Dohmen-J anssen (1999) clearly showed that the finer the sand is the larger the phase lag might be. The phase-lag parameter ( $p_{p l}$, Equation 93) is inversely related to the settling velocity. An increasing sediment transport rate with increasing grain size is clearly observed in Figure 35(a). Bed-load formulas are in general not sensitive to the grain size, with a slight proportionality for coarser sand (see also Camenen and Larroudé 2003). The effect of the phase lag is significant for both the Dibajnia and Watanabe (1992) and the modified Camenen and Larson (2005b) formulas when $d 50<0.3 \mathrm{~mm}$. The previous study by Watanabe and Isobe (1990) showed similar results for smaller wave periods ( $T_{w}=3 \mathrm{sec}$ ). For coarser grain size, the Dibajnia and Watanabe (1992) formula predicts a decrease in bed-load transport (induced by the varying $\omega_{c r}$ ) that is not observed experimentally.

The effect of grain size on the phase lag was introduced in the Camenen and Larson (2005b) formula through the settling velocity of the coefficients $\alpha_{j}$ were found to be proportional to $W_{s}^{-1.0}$. 


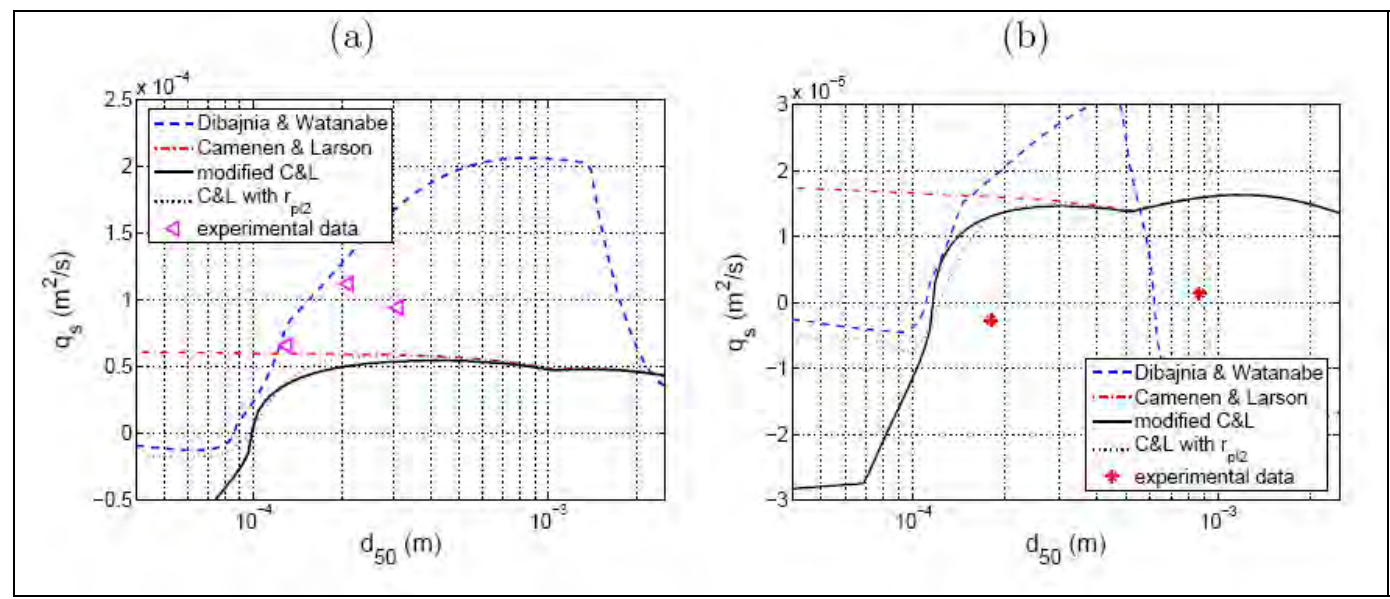

Figure 35. Influence of grain size on bed load sediment transport (details of input parameters for cases $a$ and $b$ are given in Table 11).

Table 11. Experimental conditions for studied cases on median grain size effect.

\begin{tabular}{|l|l|l|l|l|l|l|}
\hline Case & Data Set & $h(\mathrm{~m})$ & $U_{c}(\mathrm{~m} / \mathrm{sec})$ & $U_{\mathrm{w}}(\mathrm{m} / \mathrm{sec})$ & $T_{\mathrm{w}}(\mathrm{sec})$ & $r_{\mathrm{w}}(-)$ \\
\hline$(\mathrm{a})$ & Dohmen-Janssen & 0.80 & 0.24 & 1.08 & 7.2 & 0.00 \\
\hline (b) & Watanabe and Isobe & 0.31 & 0.00 & 0.69 & 3.0 & 0.20 \\
\hline
\end{tabular}

\section{Influence of wave orbital velocity}

The phase-lag effect is proportional to the wave orbital velocity, and the higher $U_{w}$ is, the larger the amount of sediment put in suspension (the available energy is greater) and the larger the sheet flow layer thickness $\left(\delta_{s}\right)$. This implies a larger delay between the instantaneous concentration and the shear stress and fluid velocity. However, the experiments by Dohmen-J anssen (1999) do not show the effect of the wave orbital velocity clearly. Nevertheless, by comparing plots (Figure 36(a), (b)) it may be observed that the increase in the sediment transport rate with $U_{w}$ is much slower for $d 50=0.13 \mathrm{~mm}$ (Figure 36(a)) compared to $d 50=0.21 \mathrm{~mm}$. Al Salem (1993) observed that the sediment transport is approximately proportional to the velocity moment to the power 3 (for a sinusoidal wave together with a current, $\left.\left\langle u^{3}\right\rangle=U_{c}^{3}+U_{c} U_{w}^{2}\right)$. If this relationship is applied to the data (Figure 36(b)) (i.e., $q_{s} \propto U_{w}^{2}$ ), it is found not to hold for case (a) where $q_{s} \propto U_{w}^{1.4}$. For the experiments of Ribberink and Chen (1993) and Ahmed and Sato (2003), where the current was negligible (Figure 36(c) and (d)), the effect of the wave orbital velocity is obvious because the direction of the sediment transport changes with an increasing $U_{w}$. 


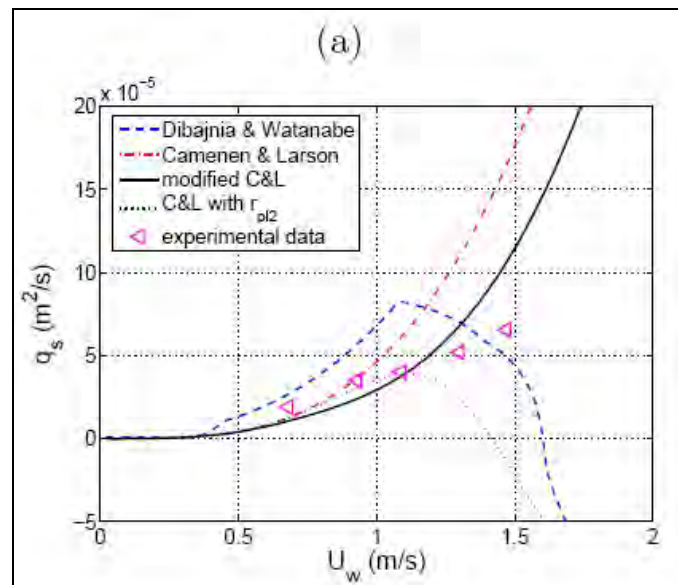

(c)

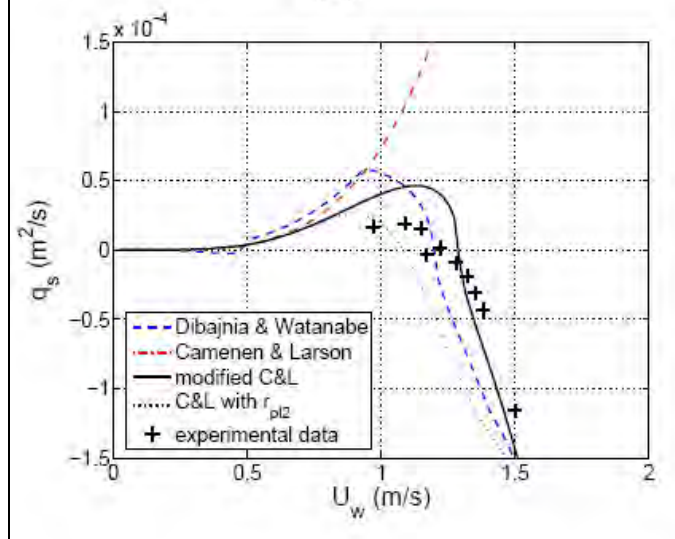

(b)

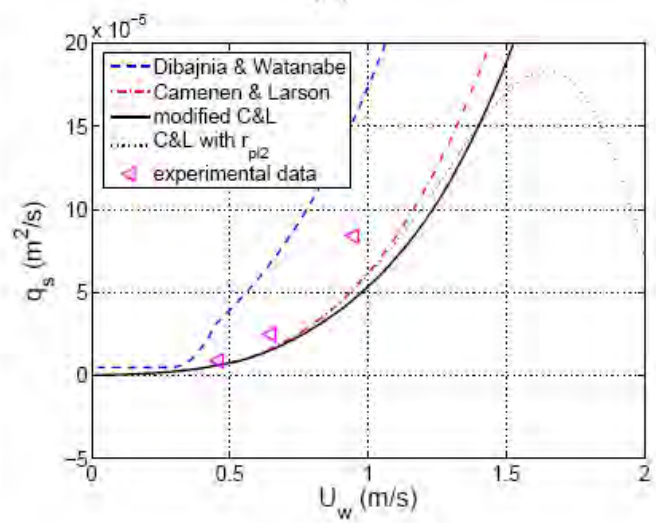

(d)

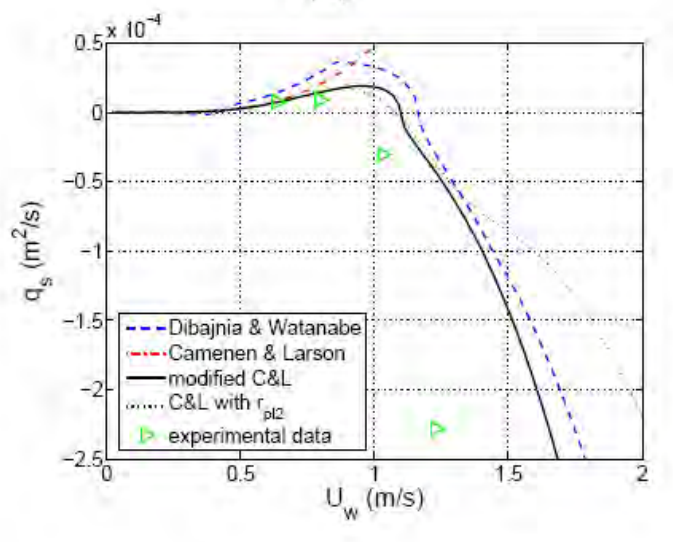

Figure 36. Influence of wave orbital velocity on sediment transport (values of input parameters for cases a, b, c, and d are given in Table 12).

The Dibajnia and Watanabe (1992) formula, as well as the Camenen and Larson (2005b) formula with the coefficient $r_{p l 2}$ (Equations 102 and 110), tends to be overly sensitive to the wave orbital velocity. Occasionally a decrease in the net sediment transport rate with an increasing wave orbital velocity is estimated, whereas the opposite behavior is observed in the experiment (Figure 36(a) and (b)). However, it seems like the sheet-flow layer and the associated phase-lag effects appear quite abruptly. Thus, predictive formulas are in general difficult to calibrate.

Table 12. Experimental conditions for studied cases on wave orbital velocity effects.

\begin{tabular}{|l|l|l|l|l|l|l|}
\hline Case & Data Set & $d_{50}(\mathrm{~mm})$ & $h(m)$ & $U_{c}(\mathrm{~m} / \mathrm{sec})$ & $T_{\mathrm{w}}(\mathrm{sec})$ & $r_{\mathrm{w}}(-)$ \\
\hline$(\mathrm{a})$ & Dohmen-Janssen & 0.13 & 0.80 & 0.24 & 7.2 & 0.00 \\
\hline (b) & Dohmen-Janssen & 0.21 & 0.80 & 0.40 & 7.2 & 0.00 \\
\hline (c) & Ahmed and Sato & 0.21 & 0.31 & 0.00 & 3.0 & 0.20 \\
\hline (d) & Ribberink and Chen & 0.13 & 0.80 & 0.03 & 6.5 & 0.25 \\
\hline
\end{tabular}


The effect of the wave orbital velocity on the phase lag was introduced in the Camenen and Larson (2005b) formula through the root-mean-square values of the velocity $U_{w j}$ (wave and current) for each half-period $T_{w j}$. The coefficients $\alpha_{j}$ were found to be proportional to $U_{w j}^{0.5}$.

\section{Influence of wave period}

The wave period is also a controlling factor for the phase lag and its effects on sediment transport: the shorter $T_{w}$ is, the larger the amount of sediment still in suspension after half a period. Indeed, the delay in sediment settling before the change in the velocity direction strongly depends on the wave period. The two experiments by Dohmen-J anssen (1999) (with $d_{50}=$ 0.13 and $0.21 \mathrm{~mm}$; Figure 37(a) and (b), respectively) clearly show an increase in sediment transport rate with an increase in wave period as soon as sheet flow occurs. A bed-load formula based on the shear stress displays an inverse behavior, because the friction coefficient $f_{w}$

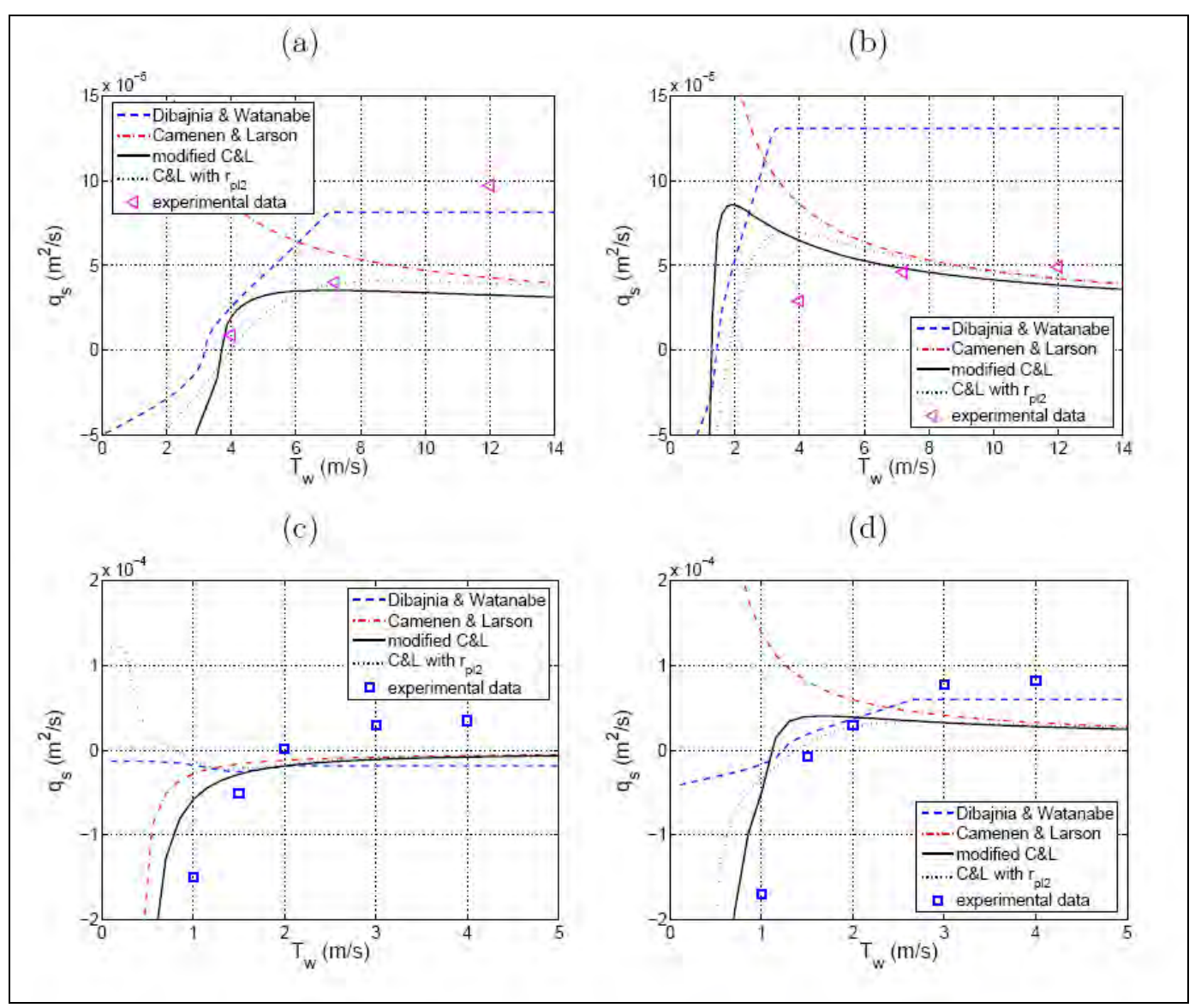

Figure 37. Influence of wave period on sediment transport (details of input parameters for cases a, b, c, and d are given in Table 13). 
Table 13. Experimental conditions for studied cases on wave period effects (for Dibajnia experiments, maximum onshore wave orbital velocity is approximately constant).

\begin{tabular}{|l|l|l|l|l|l|l|}
\hline Case & Data Set & $d_{50}(\mathrm{~mm})$ & $h(\mathrm{~m})$ & $U_{c}(\mathrm{~m} / \mathrm{sec})$ & $U_{w}(\mathrm{~m} / \mathrm{sec})$ & $r_{w}(-)$ \\
\hline (a) & Dohmen-Janssen & 0.13 & 0.80 & 0.25 & 1.06 & 0.00 \\
\hline (b) & Dohmen-Janssen & 0.21 & 0.80 & 0.25 & 1.06 & 0.00 \\
\hline (c) & Dibajnia & 0.20 & 0.22 & -0.125 & $\approx 0.80$ & $\approx 0.20$ \\
\hline (d) & Dibajnia & 0.20 & 0.22 & 0.125 & $\approx 0.80$ & $\approx 0.20$ \\
\hline
\end{tabular}

is inversely proportional to the wave period (because the boundary layer is getting thinner). The modified Camenen and Larson (2005b) formula exhibits the correct behavior, although the effect of $f_{w}$ sometimes remains larger than the introduced phase-lag parameter (Figure 37(b)). The experiments by Dibajnia (1991) (Figure 37(c) and (d)) illustrate how strong the effect of short wave periods can be. A change in the sediment transport direction for $T_{w} \approx 2 \mathrm{sec}$ (sediment transport was observed to be in the direction of the waves for $T_{w}=4 \mathrm{sec}$ and $U_{c}>-0.2 \mathrm{~m} / \mathrm{sec}$ ). However, this effect may be overestimated due to the limitations of the experimental setup. Dibajnia (1991) pointed out that the piston used in his experiment could not smoothly follow the input signal for periods $T_{w} \leq 1.5 \mathrm{sec}$. The generated vortex caused larger suspension and increased the phase-lag effects more than what normally should occur.

The Dibajnia and Watanabe (1992) and Camenen and Larson (2005b) formulas predict a negative sediment transport rate even for higher values on the wave period (Figure 37(c)). If a slight phase lag tends to decrease the absolute value of the sediment transport rate for the Dibajnia and Watanabe (1992) formula, it increases the rate for the modified Camenen and Larson (2005b) formula. This latter behavior agrees better with the measurements. However, even if it is difficult to verify the behavior with the present data, it seems like the Camenen and Larson (2005b) formula (Equations 99 and 107) is too sensitive to the wave period. In spite of this, except for case 3, the Camenen and Larson (2005b) formula with the calibrated conceptual model (Equations 101 and 110) presents better behavior than the other formulas with respect to variations in $T_{w}$.

The effect of the wave period on the phase lag was introduced in the Camenen and Larson (2005b) formula through the half-periods $T_{w j}$, and the coefficients $\alpha_{j}$ were found to be proportional to $T_{w j}^{-0.75}$. 


\section{Comparison with all data}

A comparison with all the data is presented in this section. Figure 38 and Table 14 display the overall results shown for the original Camenen and Larson (2005b) formula (Figure 38(a)), the Dibajnia and Watanabe (1992) formula (Figure 38(b)), the Camenen and Larson (2005b) formula (Equations 99 and 107 and Figure 38(c)), and the Camenen and Larson (2005b) formula with the coefficient $r_{p l}$ (Equations 102 and 110 and Figure 38(d)). It clearly shows how significant the introduction of phase lag in the formulas is. Even if the overall results (in terms of predictive skill within a factor $2, P x 2$, or $5, P x 5$ ) are not substantially improved (except for the modified Camenen and Larson (2005b) formula where results are improved by 10 percent), the general behavior of the formulas are better, and a significant decrease in the standard deviation of $\Delta q_{s}$ is obtained.

It appears that the Dibajnia and Watanabe (1992) formula and the Camenen and Larson (2005b) formula with the coefficient $r_{p l}$ tend to underestimate the absolute sediment transport rate if a phase lag occurs. However, the coefficient $r_{p l}$ (Equation 102) is sensitive to the wave asymmetry if the current is negligible. Figures 35 to 37 reveal that the Dibajnia and Watanabe (1992) formula and Camenen and Larson (2005b) formula with the coefficient $r_{p l}$ do not induce any change below a critical value (of $\left.\omega_{p l}\right)$, and then tend to abruptly decrease the sediment transport rate. On the other hand, the modified Camenen and Larson (2005b) formula produces a more gradual modification of the sediment transport rate compared to the original formula (except with regard to the wave period), which seems closer to the trend in measurements.

Finally, a comparison was made using the Camenen and Larson (2005b) formula with the parameter $r_{p l}$ proposed by Dohmen-J anssen et al. (2002) with $\delta_{s}=\alpha_{s} \theta_{c w} d_{50}$ (where $\alpha_{s}$ is a function of $d_{50}: \alpha_{s}=13$ when $d_{50}>$ $2.1 \mathrm{~mm}$, and $\alpha_{s}=35$ when $d_{50}=1.3 \mathrm{~mm}$ ). Similar to Equation 102, it improves the results if a phase lag occurs. However, the effects are often not strong enough because this analytical formula does not allow for sediment transport in the opposite direction, as was observed in the Ribberink and Chen (1993) and the Ahmed and Sato (2003) experiments. 


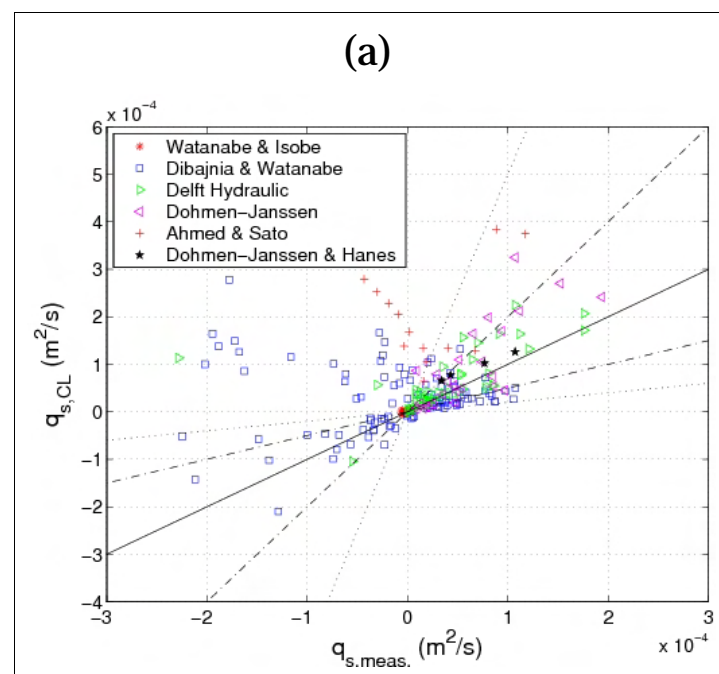

(C)

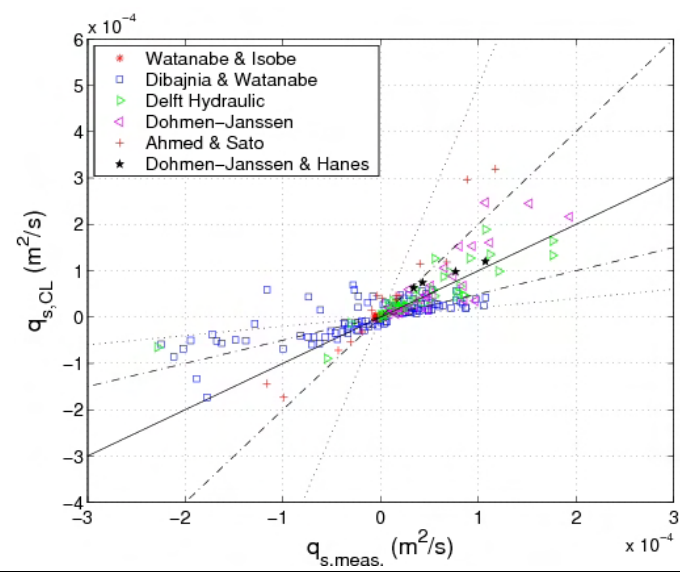

(b)

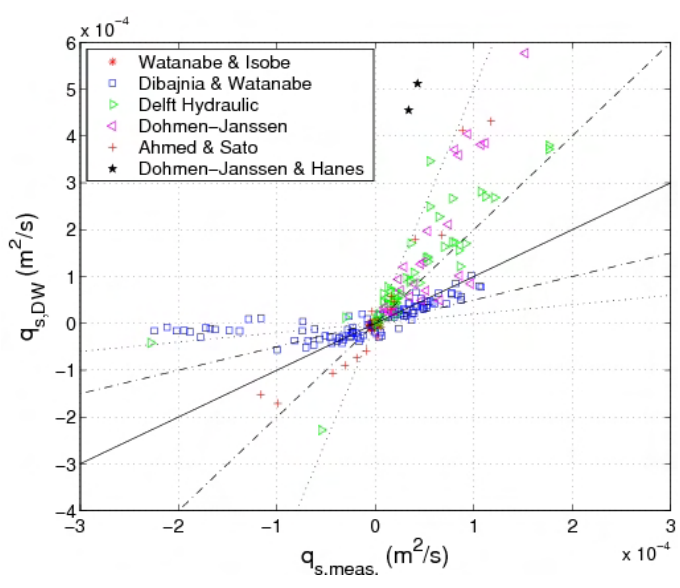

(d)

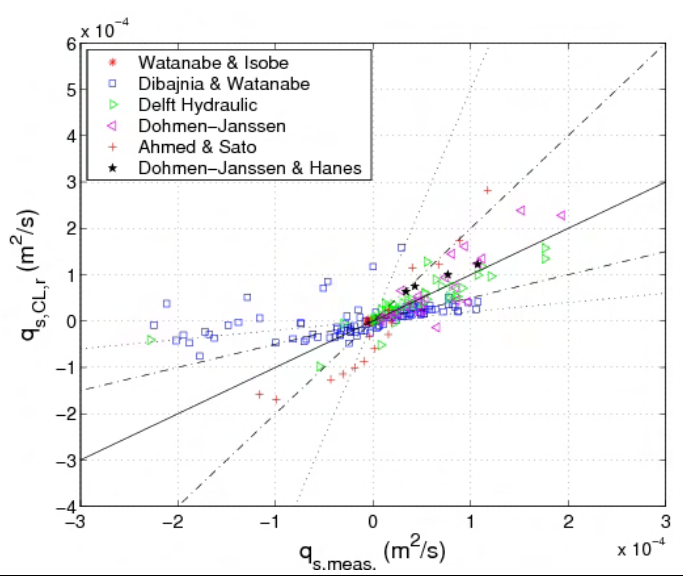

Figure 38. Comparison between predicted and measured sediment transport rate using (a) original Camenen and Larson formula, (b) Dibajnia and Watanabe formula, (c) modified Camenen and Larson formula (Equations 99 and 107), and (d) Camenen and Larson formula with coefficient $r_{p 12}$ (Equations 102 and 110).

Table 14. Prediction of bed-load transport rate within factor of 2 or 5 of measured values, together with mean value and standard deviation of $\Delta q_{\text {s. }}$.

\begin{tabular}{|l|l|l|l|l|}
\hline Author(s) & $P \times 2$ (\%) & $\begin{array}{l}P \times 5 \\
(\%)\end{array}$ & $\overline{\Delta q_{s}}$ & $\operatorname{Std}\left(\Delta q_{s}\right)$ \\
\hline Dibajnia and Watanabe (1992) & 42 & 75 & +0.11 & 7.0 \\
\hline Camenen and Larson (2005b) & 47 & 72 & +0.22 & 9.8 \\
\hline Equations 99 and 107 & 53 & 81 & -0.37 & 4.5 \\
\hline $\begin{array}{l}\text { Camenen and Larson (2005b) with Equations 102 } \\
\text { and 110 }\end{array}$ & 50 & 74 & -0.003 & 7.0 \\
\hline $\begin{array}{l}\text { Camenen and Larson (2005b) with Dohmen- } \\
\text { Janssen et al. equation (2002) for } r_{p l}\end{array}$ & 48 & 71 & -0.003 & 6.8 \\
\hline
\end{tabular}




\section{Concluding remarks on phase-lag effects}

In the present study, a large data set on sheet-flow transport was compiled and analyzed to improve the prediction of the bed-load transport rate if a phase-lag effect occurs. A simple conceptual model was proposed as well as a modification of the Camenen and Larson (2006) formula. This modification was suggested by the Dibajnia and Watanabe (1992) formula, which is the first and only formula found that takes into account the phase lag between the sediment transport rate and fluid velocity.

The conceptual model for the correction of the sediment transport rate is based on the work by Dohmen-J anssen (1999), who assumed that the sediment transport is proportional to the product of the instantaneous velocity and concentration. In turn, the instantaneous concentration is assumed to depend on the square of the instantaneous velocity with a phase $\operatorname{lag} \phi_{\mathrm{s}}>0$. This simple model allows a ratio $r_{p l}=q_{s, n e t}=q_{s, n e t, \phi_{s}}=0$ to be derived that can reach -0.35 for a sinusoidal wave and -0.5 for a secondorder Stokes wave. However, for a second-order Stokes wave, the model may diverge if the prediction is equal to zero without the phase-lag effects. The phase $\operatorname{lag} \phi_{s}$ was calibrated against data, and $\phi_{s}$ was found to be a function of the parameter $\omega_{p l}$ proposed by Dibajnia (1991). Even if significant scatter is observed, this function improves the behavior of the Camenen and Larson (2005b) formula markedly, and it is applicable to any other sediment transport formula. It also shows somewhat improved results compared to the analytical model by Dohmen-J anssen et al. (2002).

A modification of the Camenen and Larson (2005b) formula was also proposed (Equations 107 and 108). It assumes that the characteristic Shields

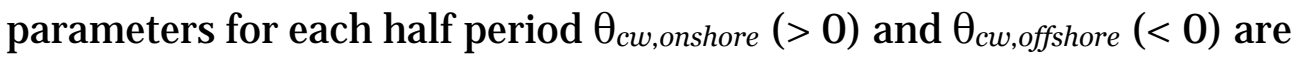
modified if the sheet flow is reached. A decrease of $\alpha_{p l} \%=\left(\alpha_{c}-\alpha_{t}\right) \%$ on $\left|\theta_{w, \text { onshore }}\right|$ and an increase of $\alpha_{p l} \%$ on $\left|\theta_{w, \text { offshore }}\right|$ introduce a general decrease in the net sediment transport rate, and it may also change the direction of the sediment transport. The coefficients $\alpha_{j}(j=c$ or $t)$ were calibrated with the compiled data and were found to be proportional to the root-mean-square velocity over the half period and inversely proportional to the settling velocity and the half period $T_{w j}$. These coefficient values are similar to those proposed by Dibajnia (1991, $\omega_{p l}$ ) and Dohmen-J anssen $\left(1999, p_{p l}\right)$. The new formula presents the best overall agreement with the data (Camenen and Larson 2006). 


\section{Suspended Load}

\section{Introduction}

The earliest transport rate formulas are mainly based on the concept that the sediment transport rate for steady uniform flow can be related to the bottom shear stress (Meyer-Peter and Müller 1948; Einstein 1950;

Engelund and Hansen 1972) assuming that bed-load transport prevails. However, if the shear stress is sufficiently large, the particles can be lifted, put in suspension, and transported in large quantities by the current. Thus, suspended load often prevails for fine sediments $\left(d_{50}<0.5 \mathrm{~mm}\right)$. The traditional approach for calculating the unsteady depth-averaged volumetric suspended load transport $q_{s s}$ is to determine the vertical distribution of suspended sediment concentration (c) and velocity $(u)$, after which the product between these two quantities is integrated through the vertical from the edge of the bed-load layer $\left(z=z_{a}\right)$ to the water surface $(z$ $=h$ ) (Van Rijn 1993), yielding:

$$
q_{s s}(t)=\int_{z_{a}}^{h} u(z, t) c(z, t) d z
$$

where $q_{s s}(t)$ is the depth-averaged instantaneous suspended load per unit width, $u(z, t)$ and $c(z, t)$ are, respectively, the horizontal velocity and the volume sediment concentration at the height $z, z_{a}$ is the level at the top of the bed-load layer, and $h(t)$ is the instantaneous water depth. A steady situation is typically assumed to simplify the problem, so time-averaged values $u(z)=\overline{u(z, t)}$ and $c(z)=\overline{c(z, t)}$ are substituted (Figure 39):

$$
q_{s s}=\int_{z_{a}}^{h} c(z) u(z) d z
$$

Therefore, an accurate estimation of the total suspended load requires accurate prediction of the mean current velocity and concentration profiles. 


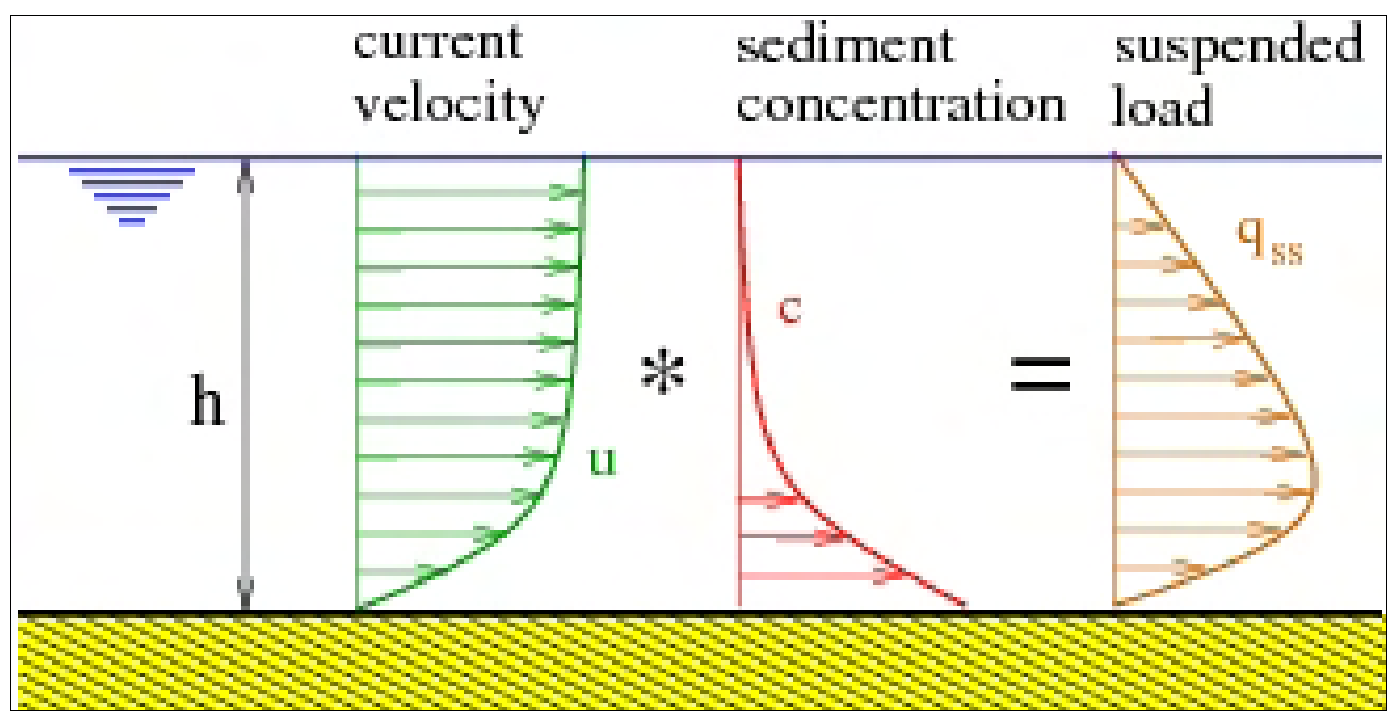

Figure 39. Computation of suspended load over depth.

In the marine coastal environment, the process of sediment transport becomes complex because of the presence of oscillatory flows and the interaction between steady and oscillatory flows. For longshore sediment transport, the effect of the short waves is typically modeled as an additional sediment stirring that increases the bed shear stress and the vertical mixing coefficient for the sediment in suspension (Bijker 1967; Watanabe 1982; Van Rijn 1993).

To describe suspended load above the wave boundary layer, some refined mathematical approaches were proposed during the last decades with sophisticated turbulence closure models (Fredsøe et al. 1985; Davies 1990; Davies et al. 1997). However, the development of practical sediment transport models still has a strong empirical character and relies heavily on physical insight combined with quantitative data obtained through laboratory and field experiments.

An essential part of morphodynamic computations for flow conditions involving suspended sediment transport is the use of a reference concentration as a bed boundary condition. Van Rijn (1984b) proposed that the reference concentration should be a function of the bed-load transport. Furthermore, the main controlling parameters for the suspended load are the settling velocity of the sediment $W_{s}$, and more specifically, the vertical sediment diffusion coefficient $\varepsilon_{v}$. The latter is examined here and compared to the Van Rijn (1984b) results. 
In the nearshore, for mild wave conditions, the bed is covered by ripples. These bed forms strongly affect the sediment transport by enhancing the suspended load, but also by modifying the direction of the sediment transport. Assuming $u(z, t)=\overline{u(z, t)}+\tilde{u}(z, t)=u(z)+\tilde{u}(z, t)$ and $c(z, t)=\overline{c(z, t)}+\tilde{c}(z, t)=c(z)+\tilde{c}(z, t)$, where $\tilde{u}(z, t)$ and $\tilde{c}(z, t)$ are the oscillatory components, the net suspended sediment transport is obtained by averaging Equation 111:

$$
\begin{gathered}
q_{s s}=<\int_{z_{a}}^{h(t)}[u(z) c(z)+\tilde{u}(z, t) \tilde{c}(z, t)] d z> \\
=q_{s s, c}+q_{s s, w}
\end{gathered}
$$

The first term on the right side (Equation 114) corresponds to the currentrelated suspended load, and the second term corresponds to the waverelated suspended load. The wave-related suspended load includes the quasi-steady suspended load due to asymmetric waves (assuming no phase lag exist between the wave velocity and the sediment concentration) and the unsteady effects due to a possible phase lag between the instantaneous velocity and concentration. Many experimental studies and most predictive models do not take into account the wave-related suspended load, although it appears to be significant for cross-shore sediment transport.

The objective of this study was to develop a reliable and general formulation for the prediction of suspended load transport valid under a wide range of fluvial and coastal conditions. For this purpose, various data sets were used for the model development including steady and oscillatory flows. The study focused on the prediction of the sediment concentration through the water column, establishing relationships for the vertical sediment diffusivity and the reference concentration. Assuming a typical velocity profile, the current-related suspended load can easily be calculated. The second part of this study focused on the possible phase lag affecting the suspended load in the wave direction due to the presence of ripples. 


\section{Equilibrium profile for suspended sediment}

Mass conservation equation

An equilibrium can exist between the settling velocity and the hydrodynamic forcing (Figure 40). The equation for the sediment concentration is derived from the mass conservation equation employed to compute changes in bottom topography:

$$
\frac{\partial c}{\partial t}+\operatorname{div} \vec{F}=0
$$

with

$$
\vec{F}=\vec{F}_{a}+\vec{F}_{s}+\vec{F}_{d}
$$

where $c$ is the sediment volume concentration (dimensionless), and $F$ is the total sediment flux (consisting of contributions from advection, settling, and diffusion):

$$
\begin{aligned}
& \vec{F}_{a}=c \vec{u}+c w \vec{e}_{z} \\
& \vec{F}_{s}=-c W_{s} \vec{e}_{z} \\
& \vec{F}_{d}=-\varepsilon_{h} \overrightarrow{\operatorname{grad}_{\mathrm{h}}}(c)-\varepsilon_{v} \frac{\partial c}{\partial z} \vec{e}_{z}
\end{aligned}
$$

with $\vec{u}$ the horizontal velocity vector, $w$ the vertical velocity, $\vec{e}_{z}$ the unit vector in the vertical, $\overrightarrow{\operatorname{grad}_{\mathrm{h}}}=\partial / \partial x+\partial / \partial y$ the horizontal gradient, and $\varepsilon_{v}$ and $\varepsilon_{h}$ the vertical and horizontal eddy diffusivity, respectively.

The bottom boundary condition yields the following relationship:

$$
-\left(\vec{F}_{s}+\vec{F}_{d}\right) \vec{n}=S
$$

where $\vec{n}$ is the unit vector perpendicular to the bottom, and $S$ the erosiondeposition flux due to suspended load. 


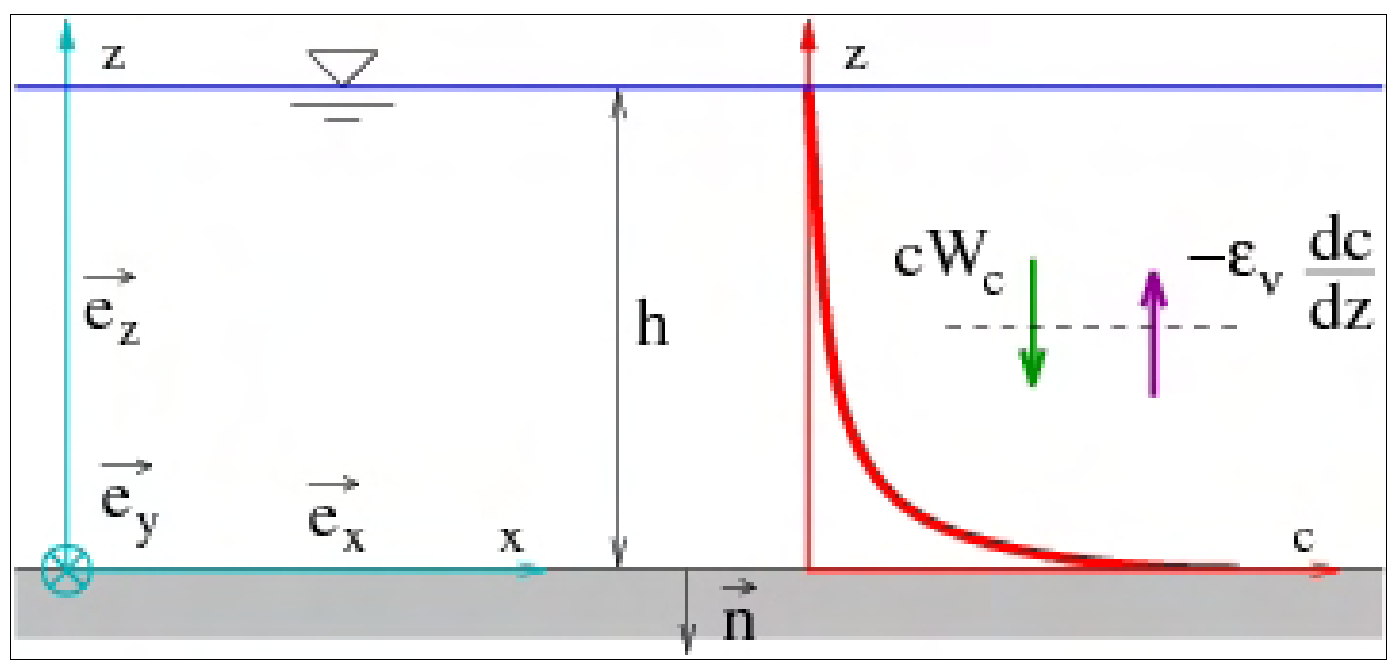

Figure 40. Concentration profile for steady conditions.

Several different expressions have been derived for the concentration profile, but most of them rely on the steady-state vertical diffusion equation (Figure 40). Under steady conditions ( $\partial c / \partial t=0, \operatorname{div} \vec{F}_{a}=0$, $\overrightarrow{\operatorname{grad}_{\mathrm{h}}}(c)=0$ and $S=0$ ), Equation 115 may be simplified to:

$$
\frac{\partial c(z)}{\partial z}+\frac{W_{s}}{\varepsilon_{v}} c(z)=0
$$

Depending on the expression selected for $\varepsilon_{v}$ analytical solutions to Equation 119 of different type may be found. Thus, if $c\left(z=z_{a}\right)=c_{a}$, where $z_{a}$ is the reference level (the maximum value of the computed roughness is often used):

$$
c(z)=c_{a} \exp \left(-\int_{z_{a}}^{z} \frac{W s}{\varepsilon_{v}} d z\right)
$$

\section{Schmidt number}

An eddy viscosity coefficient is employed for determining the mixing, or diffusion coefficient. However, the mixing of sediment is not completely analogous to the mixing of water. The vertical eddy diffusivity of particles $\varepsilon_{v}$ is then related to the vertical eddy viscosity $v_{v}$ through the Schmidt number $\sigma$ : 


$$
\sigma=\frac{\varepsilon_{v}}{v_{v}}
$$

In principle, $\sigma$ should be a constant, and $\sigma=1$. Three different processes were listed by Rose and Thorne (2001) to explain a deviation from unity:

- The first process to explain a $\sigma$-values different from unity was proposed by Sumer and Deigaard (1981) and Van Rijn (1984b). They hypothesized that the centrifugal force in a fluid eddy causes sediment grains to be thrown outside of the eddy, which increases $\sigma$.

- Another reason presented by Fredsøe and Deigaard (1994, pp. 231234) for $\sigma$ to deviate from unity might be sediment settling out of the surrounding water before the water loses its earlier composition by mixing. This effect is particularly significant for the high concentration of cohesive sediments. Lees (1981) showed that $\sigma$ displayed a decreasing trend with increasing suspended sediment concentration.

- Rose and Thorne (2001) added that the estimation of $\sigma$ may be affected by the settling velocity, which varies because of the presence of turbulence.

Based on measurements by Coleman (1981), Van Rijn (1984b) suggested the following expression for $\sigma$, which was defined as the ratio between the maximum sediment diffusivity and the maximum fluid eddy viscosity $\left(\sigma=\varepsilon_{v, \max } / v_{v, \max }\right.$ with $v_{v, \max }=0.25 \kappa u_{*} h$ for a parabolic profile):

$$
\sigma=1+2\left(\frac{W_{s}}{u_{*}}\right)^{2} \quad \text { with } \quad 0.1<\frac{W_{s}}{u_{*}}<1
$$

\section{Sediment diffusivity and concentration profiles}

The sediment diffusivity $\varepsilon_{v}$ is a fundamental parameter for the estimation of the concentration profile, and it is a function of bottom roughness and shear stress, agitation (mainly due to waves), and settling velocity. Various distributions of the sediment mixing can be found in the literature, which typically produce either an exponential or a power-law sediment concentration profile.

Exponential profile. If the sediment diffusivity $\varepsilon_{v}$ is constant ( $\varepsilon_{v} \approx$ $10^{-12} \mathrm{~m}^{2} / \mathrm{sec}$ ), an exponential profile is obtained for the mean concentration: 


$$
c(z)=c_{R} \exp \left(-\frac{W_{s}}{\varepsilon_{v}} z\right)
$$

where $c_{R}$ is the reference concentration. The ratio $W_{s} / \varepsilon_{v}$ determines suspension conditions:

if $W_{s} / \varepsilon_{v}>4$ : weak suspension,

if $W_{s} / \varepsilon_{v}<0.5$ : strong suspension.

The sediment diffusivity is often described as a function of the shear velocity and the water depth according to:

$$
\varepsilon_{v, E}=\sigma_{E} \kappa u_{*} h
$$

where $\kappa$ is Von Karman's constant $(\kappa=0.41)$, and $\sigma_{E}$ a constant consistent with the Schmidt number $\sigma$.

Power-law profile. In the coastal zone, $\varepsilon_{v}$ is typically not expected to be constant over the depth, but a function of the agitation (mainly due to waves), bottom roughness, and settling velocity. For suspended sediment in a steady current, Rouse (1938) proposed a linear equation for the sediment diffusivity:

$$
\varepsilon_{v, P}=\sigma_{P} \kappa u_{*} Z
$$

with $\sigma_{P}$ being a constant consistent with the Schmidt number $\sigma$ that Rouse assumed equal to 1 . This expression, nowadays widely used, gives the following expression for the concentration profile over depth:

$$
c(z)=c_{a}\left(\frac{z}{Z_{a}}\right)^{-W_{s} / \sigma_{p} \kappa u_{*}}
$$

The parameter $P_{R}=W_{s} /(\kappa u *)$ is commonly referred to as the Rouse parameter. It determines the shape of the suspended sediment profile, whereas the reference concentration $c_{a}$ determines the magnitude of sediment in suspension at the reference level $a$ :

- $\quad P_{R}>$ 5: near-bed suspension $(h / 10)$. 
- $5>P_{R}>2$ : suspension through bottom half of boundary layer.

- $2>P_{R}>1$ : suspension throughout the boundary layer.

- $1>P_{R}$ : uniform suspension throughout the boundary layer.

The Rouse expression may be extended to a parabolic equation for the sediment diffusivity:

$$
\varepsilon_{v, B}=\sigma_{B} \kappa u_{*} z\left(1-\frac{z}{h}\right)
$$

where $\sigma_{B}$ is a constant consistent with the Schmidt number $\sigma$. This expression implies the following solution for concentration profile over the depth:

$$
c(z)=c_{a}\left(\frac{z}{h-z} \frac{h-z_{a}}{z_{a}}\right)^{-W_{s} / \sigma_{B} \kappa u_{*}}
$$

Because $\varepsilon_{v}$ varies over the depth, different values for $\sigma_{E}, \sigma_{P}$, and $\sigma_{B}$ are obtained depending on the chosen profile. Figure 41 plots the three different analytic profiles (Equations 124, 125, and 127) with $\sigma_{E}=1 / 2 \sigma_{P}=$ $1 / 6 \sigma_{B}$ assuming that the constant value for the sediment diffusivity was obtained from an average over the depth for the linear profile and the parabolic profile.

Experimental estimation of sediment diffusivity profile. If reliable pointconcentration measurements are available, $\varepsilon_{v}$ may be calculated from (Vanoni 1946):

$$
\varepsilon_{v}=\frac{-W_{s} c}{d c / d z}
$$

However, experimental estimation of the sediment concentration often induces non-negligible errors because in most situations a maximum of 10-15 points is available over the depth, implying that the estimated slope of the resultant curve is subject to large discrepancies.

In the following, the subscript $v$ to indicate a vertical diffusivity will be dropped to simplify the notation. 


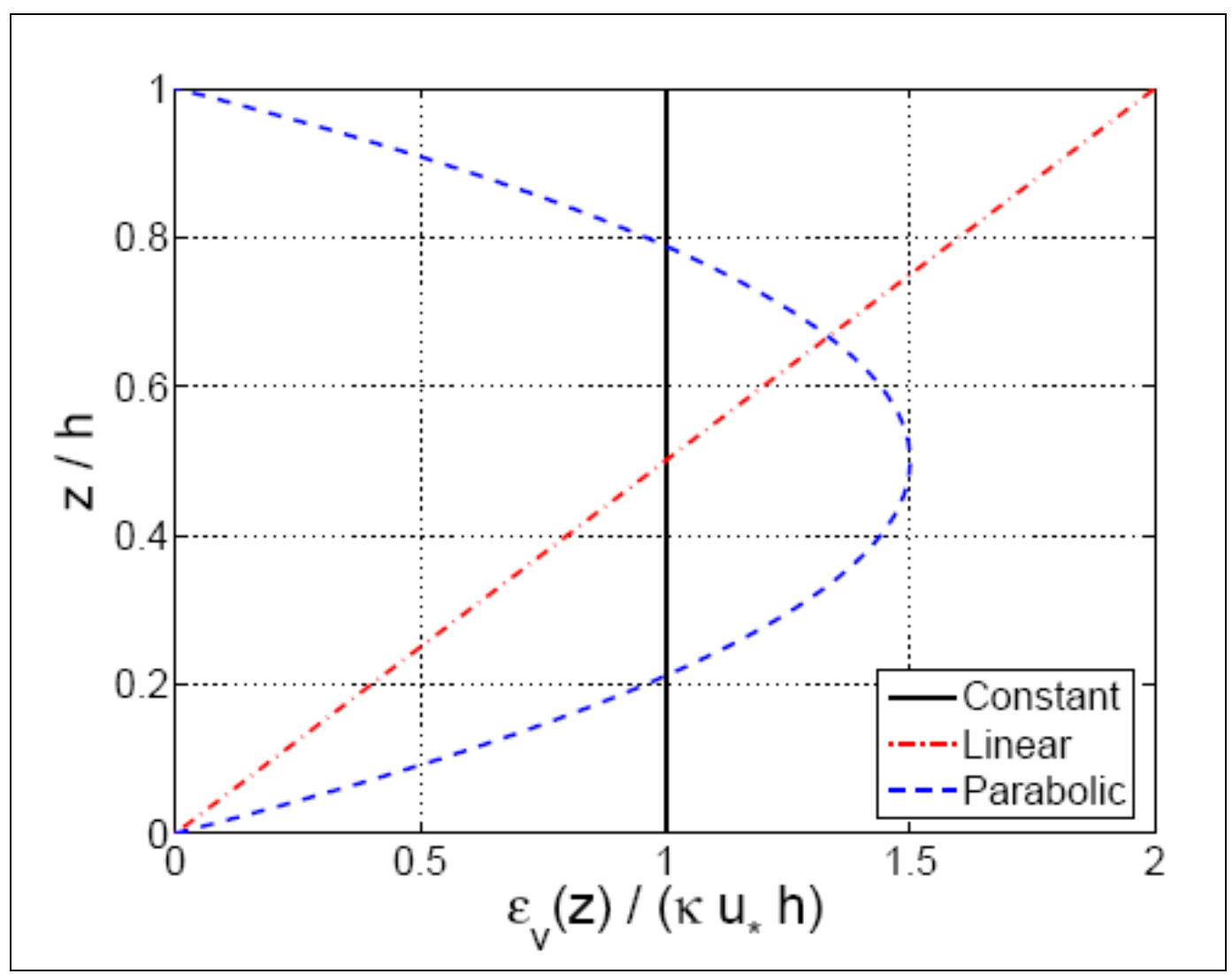

Figure 41. Three analytical relationships for vertical sediment diffusivity (Equations 124, 125, and 127) versus $z / h$ with $\sigma=\sigma_{E}=1 / 2 \sigma_{P}=1 / 6 \sigma_{B}$.

\section{Sediment diffusivity due to steady current}

\section{Experimental data}

To investigate the sediment diffusivity profile in steady conditions, available data sets covering a wide range in conditions were compiled and analyzed. Table 15 summarizes these data sets, where the types of flow motion and sediment properties are listed. For all these experiments, sand with a relative density $s=2.65$ was used. Most of these data sets were found in the SEDMOC (Van Rijn et al. 2001) data compilation.

Using the data sets summarized in Table 15, the shear velocity can be calculated from the measurements of the energy slope, but also directly from the measurements of the velocity profile. Figure 42 compares these two methods of computing the shear velocity. Even if a correlation is observed, a large scatter exists (see also Camenen et al. 2006). The energy slope 
Table 15. Data summary for suspended sediment experiments under steady currents.

\begin{tabular}{|l|l|l|l|l|l|l|l|}
\hline Author(s) & Location & Flow Type & Number & $d_{50}(\mathrm{~mm})$ & $b(\mathrm{~m})$ & $F_{r}(-)$ & $U_{*_{c}}(\mathrm{~m} / \mathrm{sec})$ \\
\hline Anderson (1942) & $\begin{array}{l}\text { Enoree River, } \\
\text { USA (1940-41) }\end{array}$ & River data & 23 & 0.7 & 15 & $0.15-0.25$ & $0.02-0.07$ \\
\hline $\begin{array}{l}\text { Barton and Lin } \\
\text { (1955) }\end{array}$ & Fort-Collins, USA & Tilting flume & 26 & 0.18 & 1.2 & $0.2-0.9$ & $0.02-0,08$ \\
\hline Laursen (1958) & $\begin{array}{l}\text { lowa, USA } \\
\text { (1961-63) }\end{array}$ & Tilting flume & 12 & $0.4,1$ & 0.9 & $0.25-0.60$ & $0.02-0.09$ \\
\hline $\begin{array}{l}\text { Scott and } \\
\text { Stephens (1966) }\end{array}$ & $\begin{array}{l}\text { Mississippi } \\
\text { River, USA } \\
\text { (1961-63) }\end{array}$ & River & 23 & 0.4 & 500 & $0.11-0.16$ & $0.05-0.13$ \\
\hline $\begin{array}{l}\text { Culbertson et al. } \\
\text { (1972) }\end{array}$ & $\begin{array}{l}\text { Rio Grande } \\
\text { River, USA } \\
\text { (1965-66) }\end{array}$ & River & 22 & $\begin{array}{l}0.18- \\
0.33\end{array}$ & 20 & $0.3-0.6$ & $0.05-0.15$ \\
\hline Voogt et al. (1991) & $\begin{array}{l}\text { Krammer Beach, } \\
\text { The Netherlands } \\
\text { (April 1987) }\end{array}$ & $\begin{array}{l}\text { Tidal } \\
\text { channel }\end{array}$ & 60 & $\begin{array}{l}0.22- \\
0.35\end{array}$ & 300 & $0.1-0.5$ & $0.03-0.15$ \\
\hline $\begin{array}{l}\text { Damgaard et al. } \\
\text { (2003) }\end{array}$ & $\begin{array}{l}\text { Wallingford, } \\
\text { Great Britain }\end{array}$ & $\begin{array}{l}\text { Duct } \\
\text { experiments }\end{array}$ & 24 & $\begin{array}{l}0.08- \\
0.20\end{array}$ & 0.6 & $0.2-0.4$ & $0.01-9.14$ \\
\hline
\end{tabular}

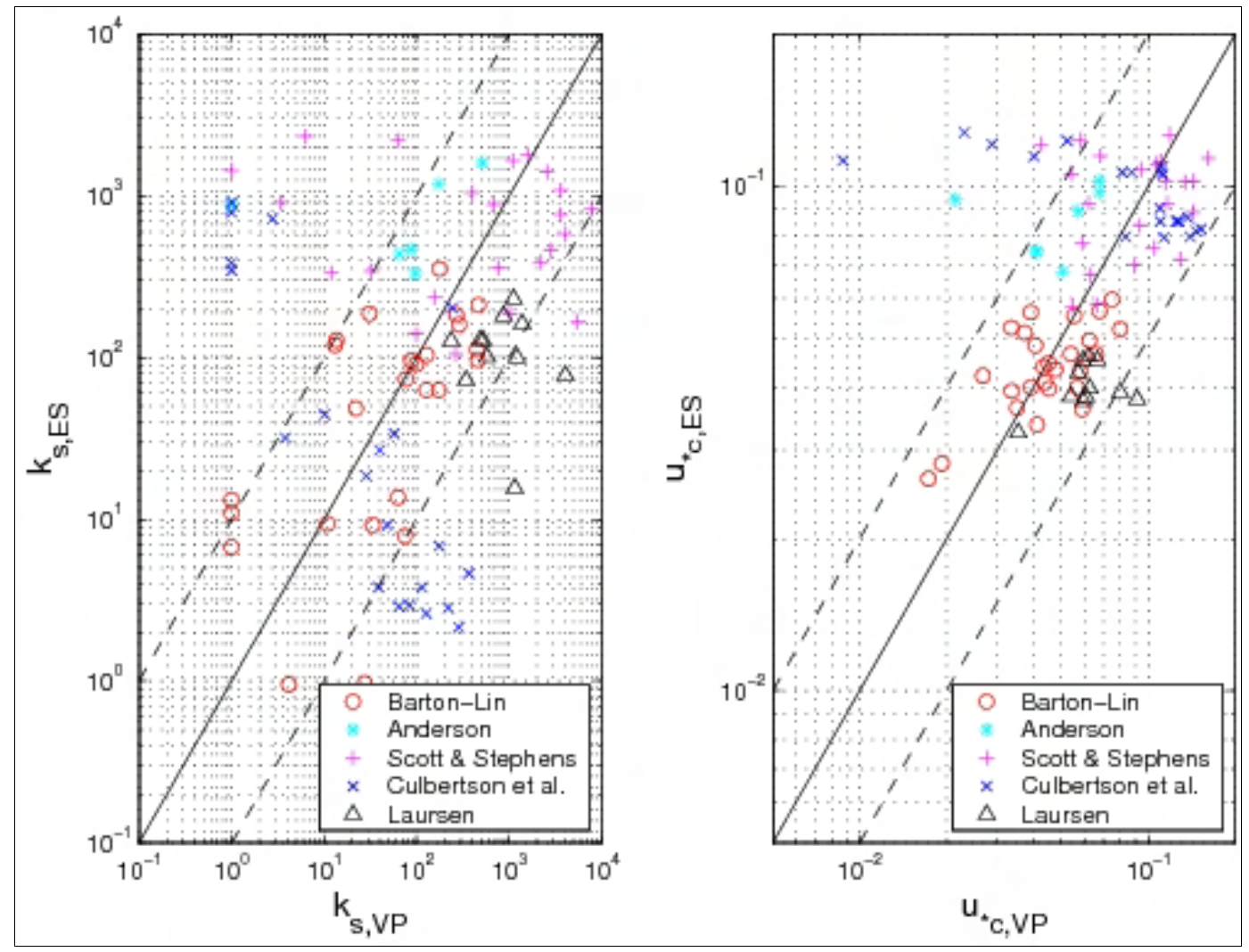

Figure 42. Comparison between "energy slope" (ES) method and "velocity profile" method (VP) to estimate the Nikuradse roughness $k_{s}$ and shear velocity $u *_{c}$. 
method appears to be more consistent, because the calculation of the shear velocity from the velocity profile shows more scatter (the number of measurement points in the velocity profile is often limited, particularly close to the bottom).

The experimental values obtained from each sediment concentration profile in the data sets are plotted (Figure 43) using Equation 129 and a pointby-point method yielding an estimate of the diffusivity according to:

$$
\varepsilon_{c, i}=\frac{-W_{s} c(i)}{\{c(i+1)-c(i-1) /[z(i+1)-z(i-1)]\}}
$$

where $i+1$ indicates consecutive experimental points along the z-axis for the concentration profile, and $\varepsilon_{c, i}$ is the estimated sediment diffusivity at level $z=z(i)$. Figure 43 confirms several results obtained previously. First, the sediment diffusivity is a linear function of the shear velocity $u^{*}$ and the water depth $h$. Nevertheless, it appears that the mean values obtained for $z \approx h / 2$ (where $\varepsilon_{c}$. is not function of $z$ ), depends on other parameters. Based on analysis of the different data set, the mean value varies from $710^{-2} \mathrm{~m}^{2} / \mathrm{sec}$ to $1 \mathrm{~m}^{2} / \mathrm{sec}$. Moreover, $\varepsilon_{c}$ appears to be an increasing function of $z$ if $z / h \leq 0.3$, a constant value for $0.3 \leq z / h \leq 0.7$, and a decreasing function of $z$ if $z / h \leq 0.7$. Thus, referring to these results and the curves obtained in Figure 41, the best of the three analytic profiles previously discussed is the parabolic profile (Equation 127). Van Rijn(1984b) also proposed the following equations to compute the current-related sediment diffusivity:

$$
\begin{aligned}
\varepsilon_{c} & =\kappa \sigma u_{*} z(1-z / h) & & \text { if } z<0.5 h \\
& =0.25 \kappa \sigma u_{*} h & & \text { if } 0.5 h<z
\end{aligned}
$$

Van Rijn (1984b) assumed that the sediment diffusivity is constant in the upper part of the water column. However, from the experimental results, a decrease is clearly observed if $z / h \leq 0.7$. Equation 131 , which produces a complex equation for the concentration profile, does not appear to improve the results compared to the parabolic profile. 

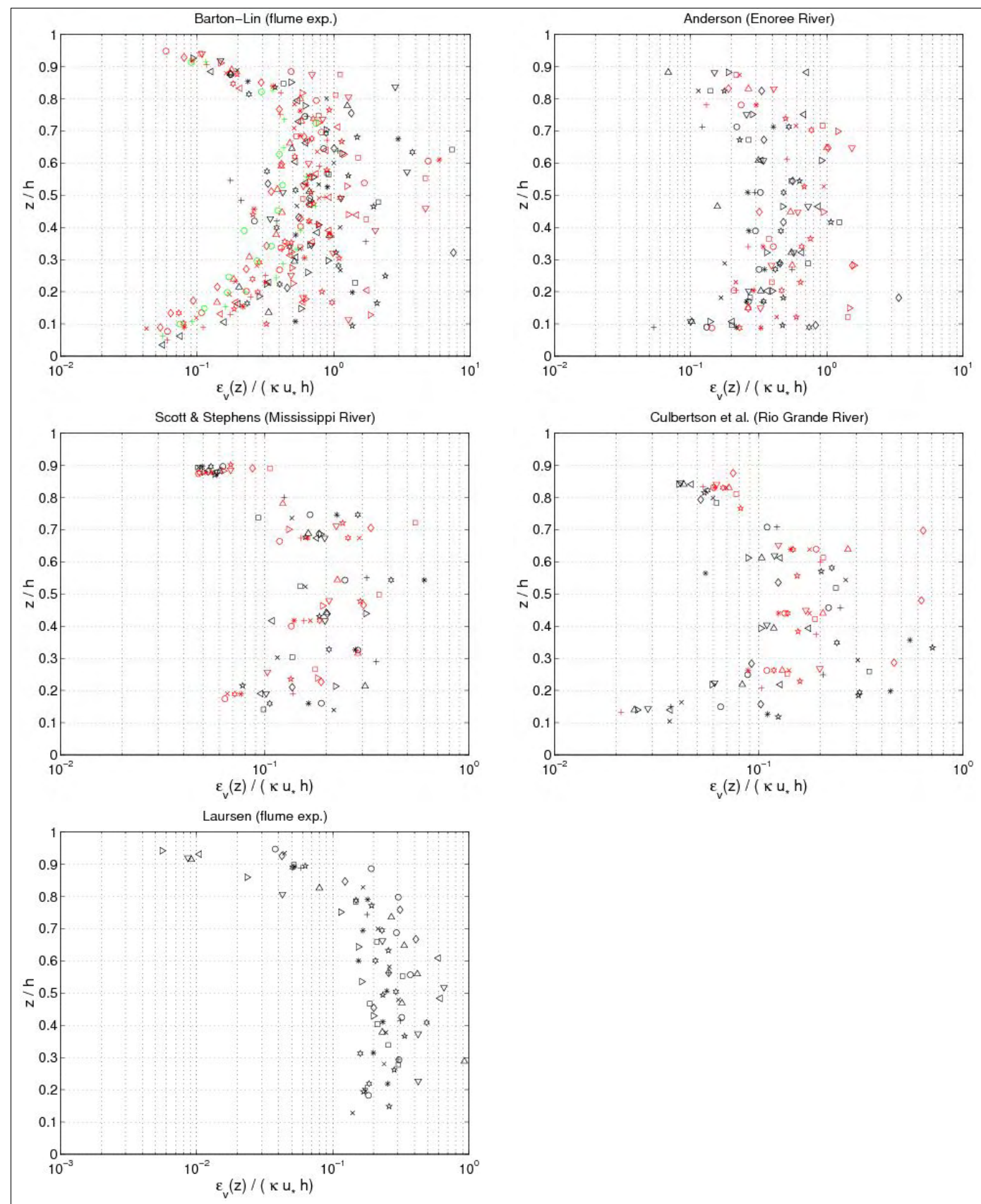

Figure 43. Vertical profile of sediment diffusivity obtained from Equation 129 using measured concentration profiles (each symbol corresponds to a particular profile).

Finally, for the data sets from Voogt et al. (1991) and Damgaard et al. (2003), measurements of the energy slope were not made, and the calculation of the shear velocity from the velocity profile shows significant scatter. Therefore, results obtained for these two data sets are not presented in Figure 43. 


\section{Shape of concentration profile}

Power-law and exponential concentration profiles were fitted to the measurements (Table 15). For a power-law profile, both linear and parabolic sediment diffusivity were examined. Some typical examples of the fit for these profiles are presented in Figure 44 for each of the data sets. Table 16 lists the percentage of sediment concentration values predicted within a range of 20 percent error of the measurements presented for the different data sets (symbolized by pred 20, meas, pred $_{20, \text { powL, }}$, and pred ${ }_{20, \text { pow }}$ for the fit of the exponential-law and the "linear" and "parabolic" power-laws, respectively in Figure 45). These calculations were also performed for sediment concentration data closer to the bed $(z<h / 3)$ and in the upper layer $(z>h / 3)$, where the velocity is approximately constant.

The main observation from this study is that there is no obvious law that fits all the measurements. However, the exponential law tends to yield better results for the upper part of the water column, whereas the linear power-law profile shows slightly better agreements closer to the bed. The parabolic power-law profile tends to produce better overall results as it presents nearly as good results as for the exponential profile in the upper part of the water column and even better results than the linear power-law profile in the lower part of the water column. This result confirms the idea of Van Rijn (1984b), who proposed an expression for the sediment diffusivity that linearly depends on the distance to the bed $z$ close to the bed and is constant for $z>h / 2$. However, large differences between the two laws are found depending on the data set. Thus, the data sets obtained from Barton and Lin (1955) and Damgaard et al. (2003) are better described by a power-law profile, whereas the data sets obtained from Voogt et al. (1991) and Laursen (1958) exhibit better agreement with an exponential profile. All the data points are plotted (Figure 45) comparing predicted and measured sediment concentrations. It can be seen that larger discrepancy appears in using the power-law profile for the three U.S. rivers data sets, whereas larger discrepancy appears using the exponential law profile with the Barton and Lin (1955) data set. 

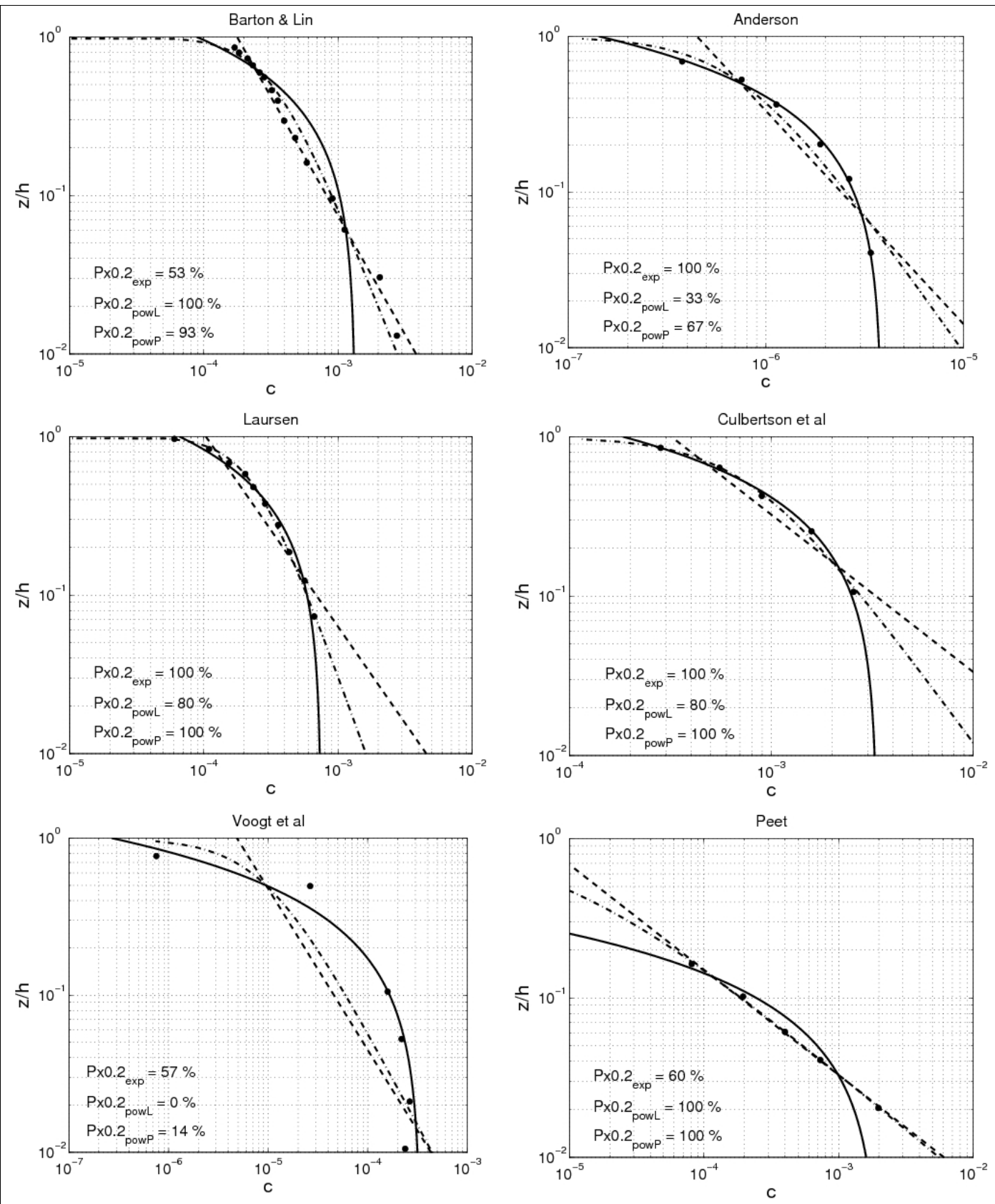

Figure 44. Examples of comparisons between predicted concentration profiles, using fitted exponential (solid line) or power-law profiles (dashed line), and measured concentration. 
Table 16. Percentage of predictions of sediment concentration within $+/-20$ percent of measured values obtained using an exponential law or power law (linear and parabolic profile) for $\varepsilon_{c}$ in fitting against data.

\begin{tabular}{|l|c|l|l|c|c|c|c|c|l|}
\hline \multirow{2}{*}{ Authors(s) } & \multicolumn{3}{|c|}{$\begin{array}{c}\text { Exponential-Law Profile } \\
(\%)\end{array}$} & \multicolumn{2}{c|}{$\begin{array}{c}\text { Power-Law Profile (linear) } \\
\text { (\%) }\end{array}$} & \multicolumn{3}{c|}{$\begin{array}{c}\text { Power-Law Profile (parab.) } \\
(\%)\end{array}$} \\
\cline { 2 - 11 } & $z$ & $z<h / 3$ & $z>h / 3$ & $z$ & $z<h / 3$ & $z>h / 3$ & $z$ & $z<h / 3$ & $z>h / 3$ \\
\hline $\begin{array}{l}\text { Barton and } \\
\text { Lin }\end{array}$ & 69.4 & 51.1 & 80.3 & 85.7 & 80.2 & 89.1 & 76.1 & 67.8 & 81.3 \\
\hline U.S. Rivers & 66.4 & 60.3 & 72.5 & 62.1 & 64.8 & 59.5 & 71.4 & 71.4 & 71.5 \\
\hline Voogt et al. & 46.6 & 38.4 & 62.2 & 36.5 & 37.6 & 34.5 & 48.9 & 44.6 & 57.1 \\
\hline Damgaard & 82.5 & 82.5 & - & 90.0 & 90.0 & - & 90.8 & 90.8 & - \\
\hline Laursen & 96.9 & 100 & 95.8 & 85.7 & 85.2 & 85.9 & 92.9 & 100 & 90.1 \\
\hline Total & 65.6 & 57.0 & 75.8 & 65.4 & 63.5 & 67.4 & 69.9 & 66.4 & 74.1 \\
\hline
\end{tabular}

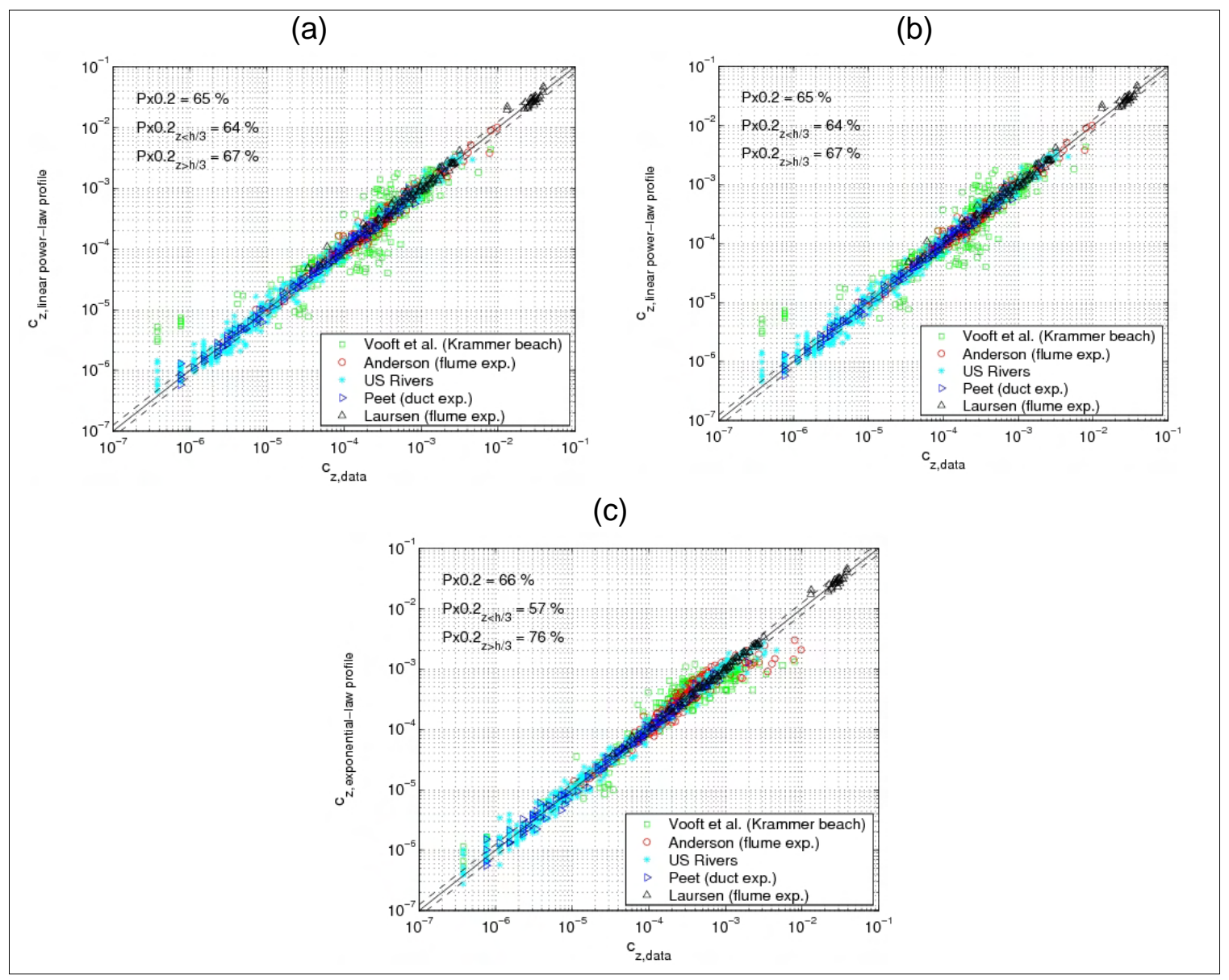

Figure 45. Comparison between predicted concentration using fitted exponential profile (a), "linear" power-law profile (b), or "parabolic" power-law profile (c), and measured concentration using all data. 
It should also be noted that the parabolic power-law profile produces a concentration equal to zero at the level $z=h$, although the two other laws allows a non-zero value. In breaking waves, the sediment diffusivity is clearly different from zero at $z=h$, which means that the parabolic powerlaw profile may not be used in the presence of breaking waves. For an expression that is applicable for both current and waves, the exponential law seems to be the most appropriate.

\section{Estimation of Schmidt number}

Based on concentration profiles obtained from the compiled data set (Table 15), the fitted power-law and exponential profiles allow for an estimate of the Schmidt number. As discussed previously, the parabolic power-law profile for the sediment diffusivity $\varepsilon_{c}$ produces the best qualitative agreement with the observed profiles. For that reason, a good correlation between $\sigma_{B}$ and $\sigma$ is expected, and $\sigma_{B}$ is calculated using Equation 128:

$$
\sigma_{B}=\frac{-W_{s}}{\alpha_{B} \kappa u_{*_{c}}}
$$

where $\alpha_{B}$ is the observed slope of the power-law relationship obtained by fitting to the data (the $\sigma_{P}$-value is obtained in a similar manner).

Some additional data were compiled (Table 17) from the studies by Rose and Thorne (2001), who also estimated $\sigma_{\mathrm{c}}$ assuming a Rouse concentration profile, and from Van Rijn (1984b), who used the Coleman (1970)data.

Table 17. Data summary for analysis on Schmidt number ( $d_{s}$ corresponds to median grain size in suspension).

\begin{tabular}{|l|l|l|l|l|l|}
\hline Author(s) & Location & Number & $d_{s}(\mathrm{~m})$ & $U_{*_{C}}(\mathrm{~m} / \mathrm{sec})$ & $h(\mathrm{~m})$ \\
\hline Coleman (1970) & Flume experiments & 16 & $1.49-2.1010^{-4}$ & $0.02-0.06$ & $0.4-0.8$ \\
\hline Whitehouse (1995) & Thames estuary, UK & 4 & $910^{-5}$ & $0.56-0.61$ & $5+/ 12$ \\
\hline Green et al. (1999) & Tanukau Harbour, NZ & 2 & $1.310^{-5}$ & $0.35,0.45$ & $12-15$ \\
\hline $\begin{array}{l}\text { Rose and Thorne } \\
(2001)\end{array}$ & Taw estuary, UK & 4 & $1.21 .310^{-4}$ & $0.41-0.63$ & $1.8-2.8$ \\
\hline
\end{tabular}


Combining data sets listed in Table 15 and the data listed in Table 17, a similar expression as the one Van Rijn (1984b) proposed was found (Figure 46):

$$
\sigma_{c} \approx \sigma_{B}=0.6+6\left(\frac{W_{s}}{u_{*_{c}}}\right)^{2}
$$

This equation produces a critical value for $W_{s} / u^{*} \approx \approx 0.25$, yielding $\sigma_{c}=1$. Up to this critical value, the centrifugal forces in the fluid eddies produce an increasing $\sigma_{c}$. Equation 133 presents improved results compared to previous predictive formulas, as shown in Table 18 and Figure 46.

Using the exponential law to estimate the sediment diffusivity, for a steady current Equation 124 reduces to:

$$
\varepsilon_{c, E}=\sigma_{E} \kappa u_{*_{c}} h \approx \frac{\sigma_{c}}{6} \kappa u_{*_{c}} h
$$

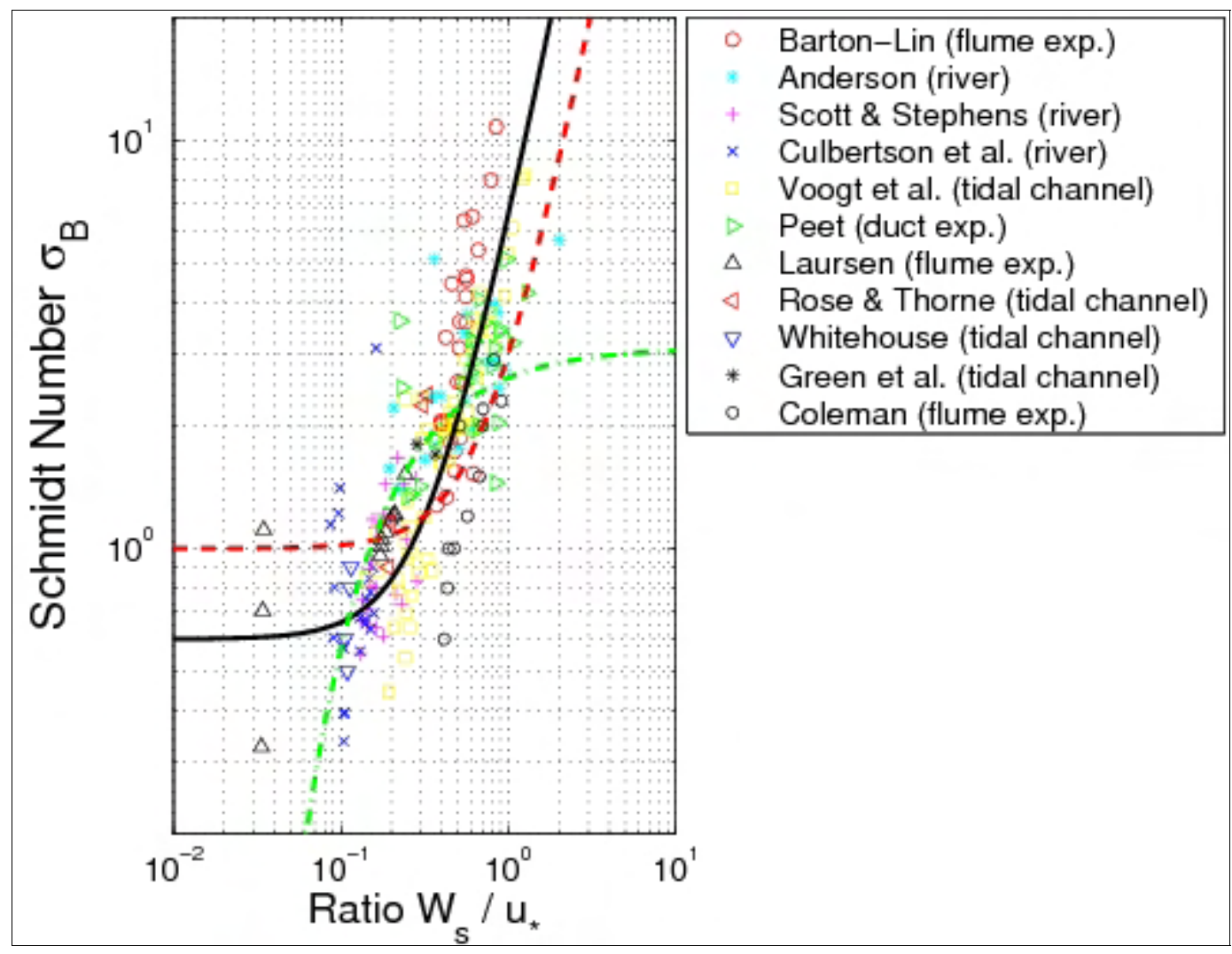

Figure 46. Estimation of Schmidt number (assumed equal to $\sigma_{B}$ ) as function of ratio $W_{s} / U *_{C}$ (solid line corresponds to Equation 133, dashed line to Equation 122, proposed by Van Rijn (1984b), and dashed-dotted line to Rose and Thorne (2001) formula). 
Table 18. Prediction of Schmidt number using parabolic profile or an exponential profile for steady current.

\begin{tabular}{|l|l|l|l|l|l|l|l|l|}
\hline \multirow{2}{*}{ Author(s) } & \multicolumn{4}{|c|}{$\sigma_{c} \approx \sigma_{B}$ (Equation 132) } & \multicolumn{3}{c|}{$\sigma_{c} \approx 6 \sigma_{E}$ (Equation 135) } \\
\cline { 2 - 10 } & $\mathrm{Px1.5 \%}$ & $\mathrm{P} \times 2 \%$ & $\begin{array}{l}\text { Mean } \\
\left(f\left(\sigma_{c}\right)\right)\end{array}$ & $\begin{array}{l}\text { Std } \\
\left(f\left(\sigma_{c}\right)\right)\end{array}$ & $\mathrm{P} \times 1.5 \%$ & $\mathrm{P} \times 2 \%$ & $\begin{array}{l}\text { Mean } \\
\left(f\left(\sigma_{c}\right)\right)\end{array}$ & $\begin{array}{l}\text { Std } \\
\left(f\left(\sigma_{c}\right)\right)\end{array}$ \\
\hline $\begin{array}{l}\text { Van Rijn } \\
\text { (Equation 123) }\end{array}$ & 53 & 78 & -0.02 & 0.25 & 54 & 84 & -0.08 & 0.22 \\
\hline Rose and Thorne & 58 & 75 & 0.02 & 0.32 & 63 & 84 & -0.04 & 0.27 \\
\hline Equation 133 & 52 & 78 & 0.03 & 0.27 & 68 & 90 & -0.02 & 0.19 \\
\hline Equation 136 & 50 & 76 & -0.04 & 0.26 & 68 & 88 & -0.09 & 0.18 \\
\hline
\end{tabular}

It is also possible to calculate the coefficient $\sigma_{E}$ for the data:

$$
\sigma_{E}=\frac{-W_{s}}{\alpha_{E} \kappa u_{*} h}
$$

where $\alpha_{E}$ is the observed slope of the exponential-law relationship. Using the discussed data sets, an expression for $\sigma_{E}$ could be $\sigma_{E}=1 / 6 \sigma_{B}$ following the results obtained for the parabolic profile where $\varepsilon_{c, E}=1 / 6 \int_{0}^{h} \varepsilon_{c, B} d z$. It may be noted that $\sigma_{E}$ is roughly six times smaller than $\sigma_{B}$ (Figure 47 ), except for the data from Voogt et al. (1991) and from Damgaard et al. (2003), where $\sigma_{E} \approx 1 / 7$ and $1 / 15 \sigma_{B}$, respectively. Similarly, $\sigma_{E}$ is roughly 2 to 3 times smaller than $\sigma_{P}$. The deviation from the theory may owe to the fact that the sediment concentration profiles were not measured through the entire water column.

Table 18 list the percentage of predicted values of the Schmidt number within a factor 0.5 and 2 (denoted Px1.5 and Px2, respectively) of the measurements presented together with the mean value and standard deviation of the function $f\left(\sigma_{c}\right)=\log \left(\sigma_{c \text {, pred }} / \sigma_{c \text {, meas }}\right)$. Equation 132 (from power law) and Equation 135 (from exponential law) were employed to estimate the Schmidt number. The spread in the results seems smaller if the Schmidt number is estimated from the exponential profile. Equation 133 yields similar results to the existing formulas if Equation 132 is employed, but improved results are obtained if Equation 135 is used (Table 18, Figure 48). 


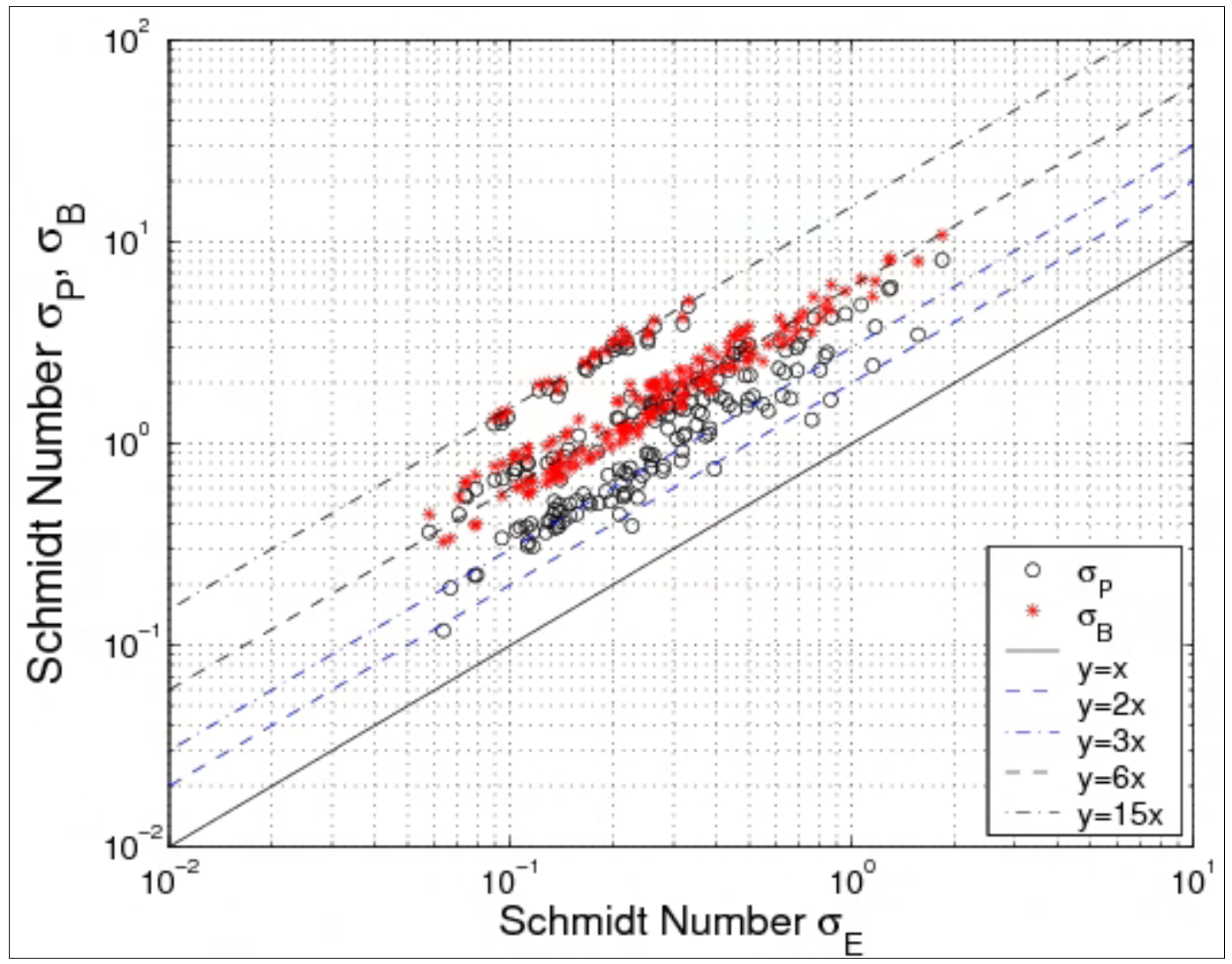

Figure 47. Estimated coefficient $\sigma_{E}$ compared to coefficients $\sigma_{P}$ and $\sigma_{B}$.

To put forward a relationship that gives physically meaningful results for all cases, it should be considered that the previous equations are correct only for $W_{s} / u_{*_{c}}<1$. For very small values of $u^{*_{c}}$, the Schmidt number must be equal to 1 , or $\sigma_{E} \approx 1 / 6 \sigma_{B}$. Thus, a new expression for $\sigma_{E}$ is proposed here (Figure 48):

$$
\sigma_{E}= \begin{cases}0.7+3.6 \sin ^{2}\left(\frac{\pi}{2} \frac{W_{s}}{u_{*_{c}}}\right) & \text { if } \frac{W_{s}}{u_{w_{c}}} \leq 1 \\ 1.0+3.3 \sin ^{2}\left(\frac{\pi}{2} \frac{u_{w_{c}}}{W_{s}}\right) & \text { if } \frac{W_{s}}{u_{w_{c}}}>1\end{cases}
$$

This equation presents slightly better results compared to Equation 133 (multiplied by 1/6, Table 18). Nevertheless, the data from the Damgaard et al. (2003) experiments are in general overestimated. This may be due to measurements only being carried out close to the bed $(z<h / 5)$. 


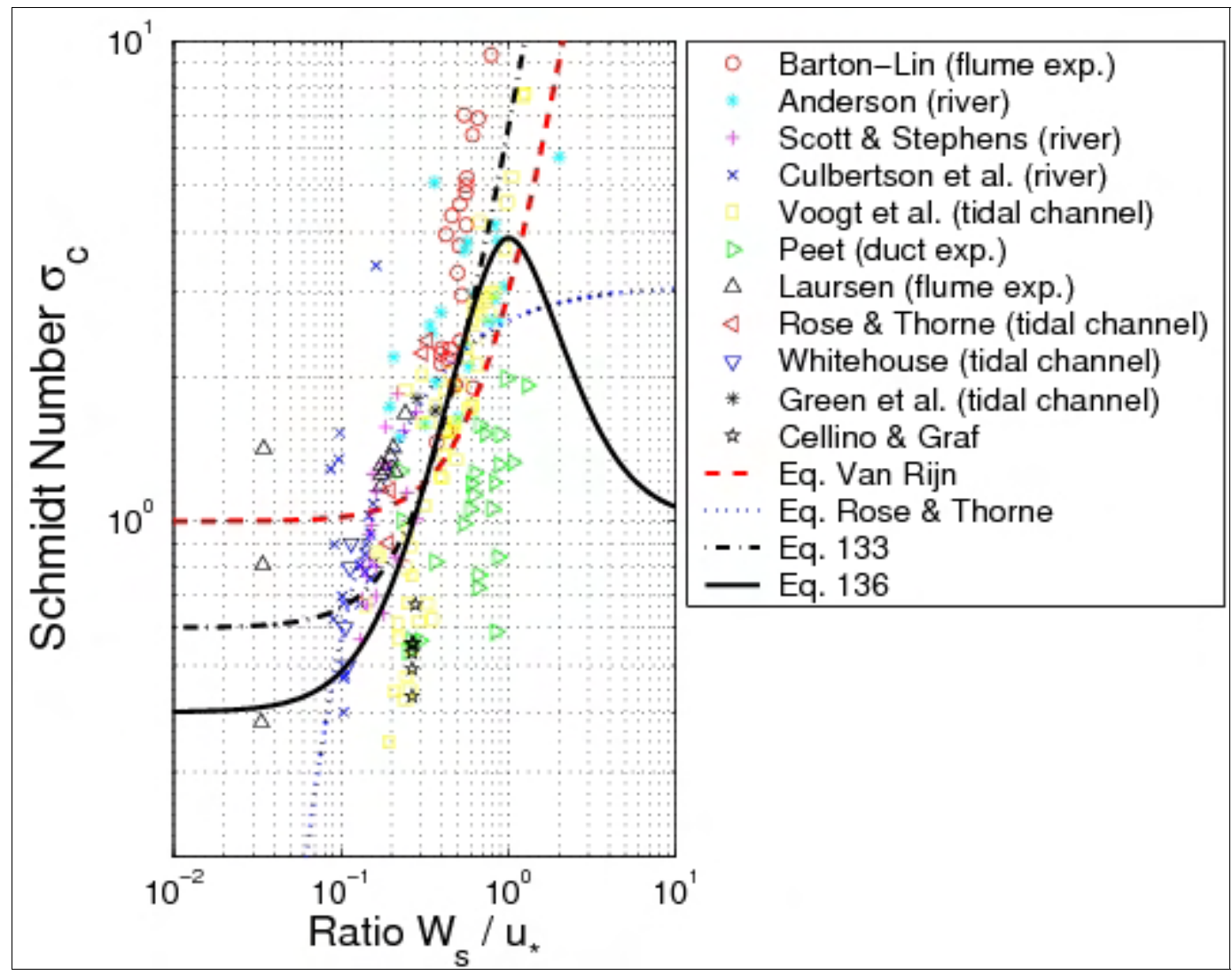

Figure 48. Estimated values of Schmidt number as function of ratio $W_{s} / u *_{c}$, together with predictive equations.

\section{Sediment diffusivity in nonbreaking waves}

The vertical distribution of suspended sediment outside the surf zone is mainly controlled by the downward settling of sediment particles, their resuspension, and the upward mixing of particles due to the generation of turbulence in boundary shear produced by friction at the seabed.

\section{Theoretical profiles}

The eddy diffusivity for suspended sediment in nonbreaking waves may be defined as a constant, a linear, or a parabolic function of the vertical position relative to the water surface (or water depth):

$$
\begin{aligned}
& \varepsilon_{w, E}=\sigma_{E} \kappa u_{* w} h \\
& \varepsilon_{w, P}=\sigma_{P} \kappa u_{* w} z
\end{aligned}
$$




$$
\varepsilon_{w, B}=\sigma_{B} \kappa u_{* w} z\left(1-\frac{z}{h}\right)
$$

where $u_{*}=\sqrt{\tau_{w} / \rho}$ is the shear velocity produced by the waves. Here, $\tau_{w}$ is the maximum shear stress due to the waves, and $\sigma_{E}, \sigma_{P}$, and $\sigma_{B}$ are the Schmidt numbers for an exponential, linear power-law, or parabolic power-law concentration profile, respectively.

\section{Estimation of sediment diffusivity profiles for oscillatory flows}

To investigate the sediment diffusivity profile under oscillatory conditions, existing data sets covering a wide range of conditions were compiled and analyzed. Table 19 summarizes the data sets employed, where the flowgeneration, sediment properties, and bed form characteristics are included. For all these experiments, sand with a relative density of $s=2.65$ was used. Most of these data sets were compiled in the SEDMOC (Van Rijn et al. 2001) data set except those from Peters (2000), Gailani and Smith (2000), Bayram et al. (2001), and Wang et al. (2002).

For the extensive field data set from Gailani and Smith (2000), only two measurements of the concentration were provided over the depth. Also, for the data set from Peters (2000), only the parameters in the fitted exponential function $\left(c_{R}\right.$ and $\varepsilon$ ) were provided. Thus, these data sets were not consulted for a detailed study of the concentration profile. In the data set from Dohmen-J anssen (1999), the measurements were made close to the bed, within the sheet-flow layer. Thus, it will not be used for the estimation of the suspended load (as it corresponds to bed load), but only for investigating the behavior of the sediment diffusivity profile.

For each data set where the number of measurements of the concentration over the water column was sufficient $(n>5)$, the parameter values obtained from each sediment concentration profile were calculated over the water column using Equation 130.

Using the data sets presented in Table 19, the total shear velocity was estimated assuming that the shear velocities produced by the current or waves, respectively, can be linearly added. For most of the cases (wave flumes), the shear stress due to the current is much smaller than the shear stress due to the waves, and thus, may be neglected. The total Nikuradse 
Table 19. Data summary for suspended sediment experiments under oscillatory flows.

\begin{tabular}{|c|c|c|c|c|c|c|c|c|c|c|}
\hline Author(s) & Location & $\begin{array}{l}\text { Flow } \\
\text { Facility }\end{array}$ & Number & $d_{50}(\mathrm{~mm})$ & $h(\mathrm{~m})$ & $U_{c}(\mathrm{~m} / \mathrm{sec})$ & $\begin{array}{l}U_{w^{1}} \\
(\mathrm{~m} / \mathrm{sec})\end{array}$ & $T_{w^{1}}(\mathrm{sec})$ & $H_{r}(\mathrm{~m})$ & $L_{r}(\mathrm{~m})$ \\
\hline $\begin{array}{l}\text { Bosman (1982) } \\
\text { and Steetzel } \\
(1985)\end{array}$ & $\begin{array}{l}\text { DHL, The } \\
\text { Netherlands }\end{array}$ & $\begin{array}{l}\text { Wave } \\
\text { flume }\end{array}$ & $70(50)^{*}$ & 0.10 & $\begin{array}{l}0.1- \\
0.65\end{array}$ & $0.10-0.32$ & $\begin{array}{l}0.13- \\
0.30\end{array}$ & $1.4-2.0$ & $\begin{array}{l}0.01- \\
0.03\end{array}$ & 0.08 \\
\hline Nielsen (1984) & $\begin{array}{l}\text { Australian } \\
\text { beaches (1980- } \\
82 \text { ), Australia }\end{array}$ & Field & $65(39)^{*}$ & $\begin{array}{l}0.11- \\
0.62\end{array}$ & $\begin{array}{l}0.8- \\
1.8\end{array}$ & $0-0.54$ & $\begin{array}{l}0.28- \\
0.80\end{array}$ & $5.3-14.4$ & $\begin{array}{l}0^{2}- \\
0.20\end{array}$ & $0^{2}-1.5$ \\
\hline $\begin{array}{l}\text { Steetzel (1984) } \\
\text { and Van der } \\
\text { Velden (1986) }\end{array}$ & $\begin{array}{l}\text { DHL, The } \\
\text { Netherlands }\end{array}$ & $\begin{array}{l}\text { Small } \\
\text { water } \\
\text { tunnel }\end{array}$ & $\begin{array}{l}259 \\
(259)^{*}\end{array}$ & $\begin{array}{l}0.10- \\
0.36\end{array}$ & 0.4 & 0 & $\begin{array}{l}0.07- \\
0.65\end{array}$ & $1.0-7.0$ & $\begin{array}{l}0.005- \\
0.1\end{array}$ & $\begin{array}{l}0.011- \\
0.55\end{array}$ \\
\hline $\begin{array}{l}\text { Dette and } \\
\text { Uliczka (1986) }\end{array}$ & $\begin{array}{l}\text { Hannover, } \\
\text { Germany }\end{array}$ & $\begin{array}{l}\text { Large } \\
\text { wave } \\
\text { flume }\end{array}$ & $11(0)^{*}$ & 0.33 & $\begin{array}{l}0.9- \\
2.6\end{array}$ & 0 & $\begin{array}{l}0.95- \\
1.65\end{array}$ & 6.0 & -3 & -3 \\
\hline Kroon (1991) & $\begin{array}{l}\text { Egmond Beach } \\
\text { (1989-90), The } \\
\text { Netherlands }\end{array}$ & Field & $31(11)^{*}$ & $\begin{array}{l}0.30- \\
0.47\end{array}$ & $\begin{array}{l}0.4- \\
1.5\end{array}$ & $\begin{array}{l}-0.55- \\
0.97\end{array}$ & $\begin{array}{l}0.20- \\
0.91\end{array}$ & 3.1 - 12.6 & $\begin{array}{l}0.005- \\
0.05\end{array}$ & $\begin{array}{l}0.15- \\
0.75\end{array}$ \\
\hline Havinga (1992) & $\begin{array}{l}\text { VinjeBasin, Delft, } \\
\text { The Netherlands }\end{array}$ & Basin & $28(28)^{*}$ & 0.10 & $\begin{array}{l}0.40- \\
0.43\end{array}$ & $\begin{array}{l}0.10- \\
0.32\end{array}$ & $0-0.80$ & $2.1-2.3$ & -3 & -3 \\
\hline $\begin{array}{l}\text { Ribberink and Al } \\
\text { Salem (1994) }\end{array}$ & $\begin{array}{l}\text { DHL, Delft, The } \\
\text { Netherlands }\end{array}$ & $\begin{array}{l}\text { Large } \\
\text { water } \\
\text { tunnel }\end{array}$ & $71(71)^{*}$ & 0.21 & 0.8 & 0 & $0.2-1.5$ & $2.0-12.0$ & $\begin{array}{l}0^{2}- \\
0.35\end{array}$ & $0^{2}-3.0$ \\
\hline $\begin{array}{l}\text { Dohmen-Janssen } \\
\text { (1999) }\end{array}$ & $\begin{array}{l}\text { DHL, Delft, The } \\
\text { Netherlands }\end{array}$ & $\begin{array}{l}\text { Large } \\
\text { water } \\
\text { tunnel }\end{array}$ & 9 & $\begin{array}{l}0.13- \\
0.32\end{array}$ & 0.80 & $\begin{array}{l}0.03- \\
0.43\end{array}$ & $\begin{array}{l}0.59- \\
1.07\end{array}$ & $4.0-12.0$ & $0^{2}$ & $0^{2}$ \\
\hline $\begin{array}{l}\text { Chung et al. } \\
\text { (2000) }\end{array}$ & $\begin{array}{l}\text { Deltaflume, DHL, } \\
\text { Delft, The } \\
\text { Netherlands }\end{array}$ & $\begin{array}{l}\text { Large } \\
\text { wave } \\
\text { flume }\end{array}$ & $19(19)^{*}$ & $\begin{array}{l}0.16- \\
0.33\end{array}$ & $\begin{array}{l}3.5- \\
4.5\end{array}$ & $\begin{array}{l}-0.04-- \\
0.02\end{array}$ & $\begin{array}{l}0.56- \\
0.67\end{array}$ & $6.6-7.1$ & $\begin{array}{l}0.03- \\
0.05\end{array}$ & $\begin{array}{l}0.25- \\
0.75\end{array}$ \\
\hline Peters (2000) & $\begin{array}{l}\text { Großen } \\
\text { Wellenkanal } \\
\text { GWK, Hannover, } \\
\text { Germany }\end{array}$ & $\begin{array}{l}\text { Large } \\
\text { wave } \\
\text { flume }\end{array}$ & $349(0)^{*}$ & $\begin{array}{l}0.12- \\
0.33\end{array}$ & $\begin{array}{l}0.4- \\
2.4\end{array}$ & $\begin{array}{l}-0.35- \\
0.14\end{array}$ & $\begin{array}{l}0.49- \\
1.24\end{array}$ & 5.5 & & \\
\hline $\begin{array}{l}\text { Gailani and } \\
\text { Smith (2000) }\end{array}$ & $\begin{array}{l}\text { Mouth Columbia } \\
\text { River, } \\
\text { Washington, USA }\end{array}$ & Field & $\begin{array}{l}818 \\
(818)^{*}\end{array}$ & 0.22 & $\begin{array}{l}16.6- \\
19.5\end{array}$ & $0-0.88$ & $\begin{array}{l}0.03- \\
1.49\end{array}$ & $4.8-21.3$ & -3 & -3 \\
\hline $\begin{array}{l}\text { Voulgaris and } \\
\text { Collins (2000) }\end{array}$ & $\begin{array}{l}\text { Bournemouth } \\
\text { Beach, Caswell } \\
\text { Bay, Rhossili } \\
\text { Bay, UK }\end{array}$ & Field & $12(12)^{*}$ & $\begin{array}{l}0.21 \\
0.26,0.33\end{array}$ & $\begin{array}{l}0.4- \\
2.1\end{array}$ & $\begin{array}{l}0.01- \\
0.10\end{array}$ & $\begin{array}{l}0.16- \\
0.40\end{array}$ & $3.2-9.1$ & -3 & -3 \\
\hline $\begin{array}{l}\text { SEDMOC data } \\
\text { set (Van Rijn et } \\
\text { al. 2001) - } \\
\text { Vessem- }\end{array}$ & $\begin{array}{l}\text { Eastern Scheldt } \\
\text { estuary (1983- } \\
\text { 84), The } \\
\text { Netherlands }\end{array}$ & Field & $70(70)^{*}$ & 0.15 & $\begin{array}{l}0.7- \\
4.0\end{array}$ & $\begin{array}{l}0.05- \\
0.65\end{array}$ & $\begin{array}{l}0.02- \\
0.40\end{array}$ & $2.0-3.2$ & 0.05 & -3 \\
\hline $\begin{array}{l}\text { SEDMOC data } \\
\text { set (Van Rijn et } \\
\text { al. 2001) }\end{array}$ & $\begin{array}{l}\text { Grote Speurwerk } \\
\text { (35 m), DUT, } \\
\text { Delft, The } \\
\text { Netherlands }\end{array}$ & $\begin{array}{l}\text { Wave } \\
\text { flume }\end{array}$ & $125(81)^{*}$ & $\begin{array}{l}0.10- \\
0.22\end{array}$ & $\begin{array}{l}0.29- \\
0.60\end{array}$ & $\begin{array}{l}0.07- \\
0.45\end{array}$ & $0.17-0.55$ & $1.2-2.7$ & $\begin{array}{l}0.002- \\
0.029\end{array}$ & $\begin{array}{l}0.006- \\
0.20\end{array}$ \\
\hline
\end{tabular}




\begin{tabular}{|c|c|c|c|c|c|c|c|c|c|c|}
\hline Author(s) & Location & $\begin{array}{l}\text { Flow } \\
\text { Facility }\end{array}$ & Number & $d_{50}(\mathrm{~mm})$ & $h(m)$ & $U_{c}(\mathrm{~m} / \mathrm{sec})$ & $\begin{array}{l}U_{w^{1}} \\
(\mathrm{~m} / \mathrm{sec})\end{array}$ & $T_{w^{1}}(\mathrm{sec})$ & $H_{r}(\mathrm{~m})$ & $L_{r}(m)$ \\
\hline $\begin{array}{l}\text { SEDMOC data } \\
\text { set (Van Rijn et } \\
\text { al. 2001) }\end{array}$ & $\begin{array}{l}\text { Grote Speurwerk } \\
(45 \text { m), DUT, } \\
\text { Delft, The } \\
\text { Netherlands }\end{array}$ & $\begin{array}{l}\text { Wave } \\
\text { flume }\end{array}$ & $62(62)^{*}$ & $\begin{array}{l}0.15- \\
0.29\end{array}$ & $\begin{array}{l}0.49- \\
0.55\end{array}$ & $\begin{array}{l}0.16- \\
0.35\end{array}$ & $\begin{array}{l}0.14- \\
0.60\end{array}$ & $2.4-2.8$ & -3 & -3 \\
\hline $\begin{array}{l}\text { SEDMOC data } \\
\text { set (Van Rijn et } \\
\text { al. 2001) }\end{array}$ & $\begin{array}{l}\text { Deltaflume, DHL, } \\
\text { Delft, The } \\
\text { Netherlands }\end{array}$ & $\begin{array}{l}\text { Large } \\
\text { wave } \\
\text { flume }\end{array}$ & $57(30)^{*}$ & $\begin{array}{l}0.19- \\
0.24\end{array}$ & $\begin{array}{l}0.7- \\
3.4\end{array}$ & $-0.18-0$ & $\begin{array}{l}0.67- \\
1.46\end{array}$ & $2.6-5.0$ & $\begin{array}{l}0^{2}- \\
0.04\end{array}$ & $0^{2}-1.0$ \\
\hline $\begin{array}{l}\text { Bayram et al. } \\
\text { (2001) }\end{array}$ & $\begin{array}{l}\text { Sandy-Duck } \\
\text { (1996-98), SC, } \\
\text { USA }\end{array}$ & Field & $66(25)^{*}$ & $\begin{array}{l}0.18- \\
0.20\end{array}$ & $\begin{array}{l}1.2- \\
8.6\end{array}$ & $\begin{array}{l}0.04- \\
1.32\end{array}$ & $0.71-2.13$ & $8.0-12.8$ & -3 & -3 \\
\hline $\begin{array}{l}\text { Wang et al. } \\
(2002)\end{array}$ & $\begin{array}{l}\text { LSTF, Vicksburg, } \\
\text { MS, USA }\end{array}$ & $\begin{array}{l}\text { Large } \\
\text { basin }\end{array}$ & $14(0)^{*}$ & 0.22 & $\begin{array}{l}0.10- \\
0.40\end{array}$ & $0-0.18$ & $\begin{array}{l}0.27- \\
0.45\end{array}$ & $1.5,3.0$ & -3 & -3 \\
\hline \multicolumn{11}{|c|}{$\begin{array}{l}\text { NOTE: Delft Hydraulics Laboratory (DHL), Delft University of Technology (DUT), Large-scale Sediment Transport Facility (LSTF). } \\
\text { * Number of nonbreaking cases. } \\
1 \text { Random waves, } U_{s} \text { is computed from the root-mean-square wave height and } T_{w}=T_{p .} . \\
2 \text { Flat bed. } \\
{ }^{3} \text { Not available. }\end{array}$} \\
\hline
\end{tabular}

roughness $k_{s t}$ was estimated using the method proposed by Soulsby (1997) by adding the grain-related, form-drag, and sediment transport roughness, $k_{s, g}, k_{s, r}$, and $k_{s, s f}$, respectively, assuming that the effects of waves prevail:

$$
k_{s, t}=k_{s, g}+k_{s, r}+k_{s, s f}
$$

where $k_{s, s f}$ was obtained from the Wilson (1966, 1989a, b) formula and $k_{s, r}$ using the following formula:

$$
k_{s, r}=\alpha_{r} \frac{H_{r}^{2}}{L_{r}}
$$

where $H_{r}$ and $L_{r}$ are the ripple height and length, and $\alpha_{r}$ is a constant $\left(\alpha_{r}=0.25\right)$. If the ripple characteristics were not measured, the Van Rijn (1989) formulas, proposed for irregular waves, were used (Equation 38).

The uncertainty involved in estimating the Nikuradse roughness and the ripple geometry explains the large scatter (Figures 49 and 50) as compared to the steady flow data previously discussed.

From Figures 49 and 50, three regions are observed regarding the sediment diffusivity profile under oscillatory flow. Close to the bottom, the sediment diffusivity reaches a minimum value and seems to be constant 
(the data sets from "Schelde Flume," Ribberink and Al Salem, DohmenJ anssen). Above this region, the sediment diffusivity is a linear function of the elevation $z / h$ until it reaches a maximum value (the data sets from "Grote Speurwerk Flume," Ribberink and Al Salem (1994), Dette and Ulickza (1986); and “Delta Flume”). Thus, as proposed by Van Rijn (1989), the sediment diffusivity for non-breaking waves may be described by the following equations:

$$
\begin{array}{ll}
Z \leq \delta_{m} & \varepsilon_{w}=\varepsilon_{w, \text { bed }} \\
Z \geq z_{w} & \varepsilon_{w}=\varepsilon_{w, \max } \\
\delta_{s}<Z<Z_{w} & \varepsilon_{w}=\varepsilon_{w, \text { bed }}+\left(\varepsilon_{w, \max }-\varepsilon_{w, \text { bed }}\right) \frac{Z-\delta_{m}}{Z_{w}-\delta_{m}}
\end{array}
$$

where $\delta_{m}$ is the moving mixing layer (found to be equal to about three times the ripple height in the ripple regime, and three times the boundary layer thickness in the sheet-flow regime), and $z_{w}$ is the elevation from where the sediment diffusivity appears to be approximately constant over the depth (Van Rijn 1989 proposed $z_{w}=0.5 h$ ). Kosyan (1985) showed that the monotonic increase of the diffusion coefficient may be caused by to the orbital motion of the waves, whereas the constant value at the bottom may be caused by the friction between the moving fluid mass and the rough bottom.

From the data set of Dohmen-J anssen (1999), measurements were provided close to and inside the fixed bed. The calculation of the sediment diffusivity in this region is much more difficult because of the estimation of the hindered settling velocity (close to the bed, $W_{s}$ decreases markedly with increasing sediment concentration). The Richardson and Zaki (1954) equation (Equation 10 with $\alpha=4$ ) limits the peak of the sediment diffusivity close to the bottom compared to a constant value for $W_{s}$. It appears that the sediment diffusivity increases strongly after entering the fixed bed. However, the advection-diffusion equation (Equation 115 to 117) is not valid anymore. 

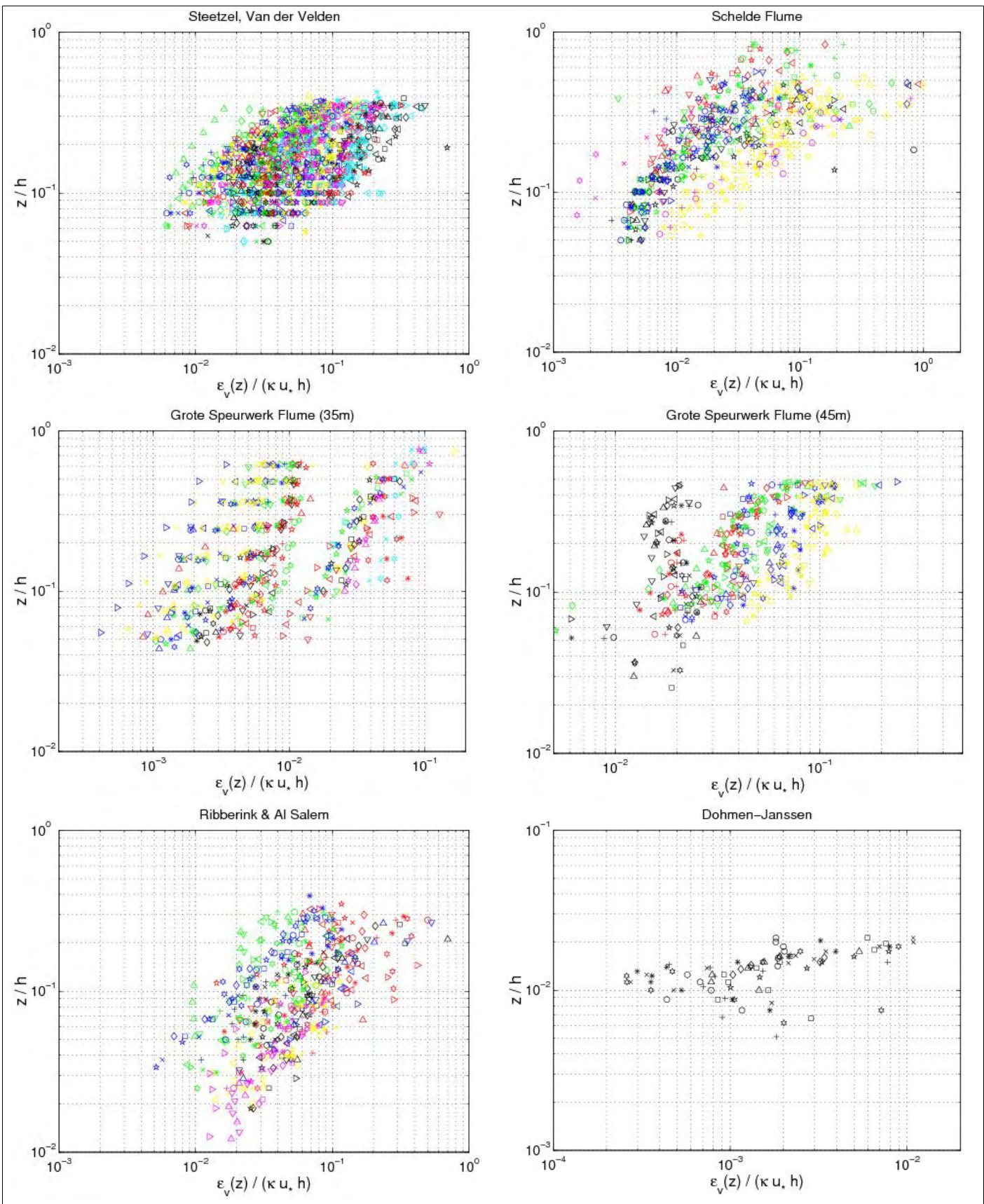

Figure 49. Vertical profiles of eddy diffusivity obtained from Equation 130 using measured concentration profiles with interaction between nonbreaking waves and current (data from wave flumes and water tunnels; each symbol corresponds to particular profile). 

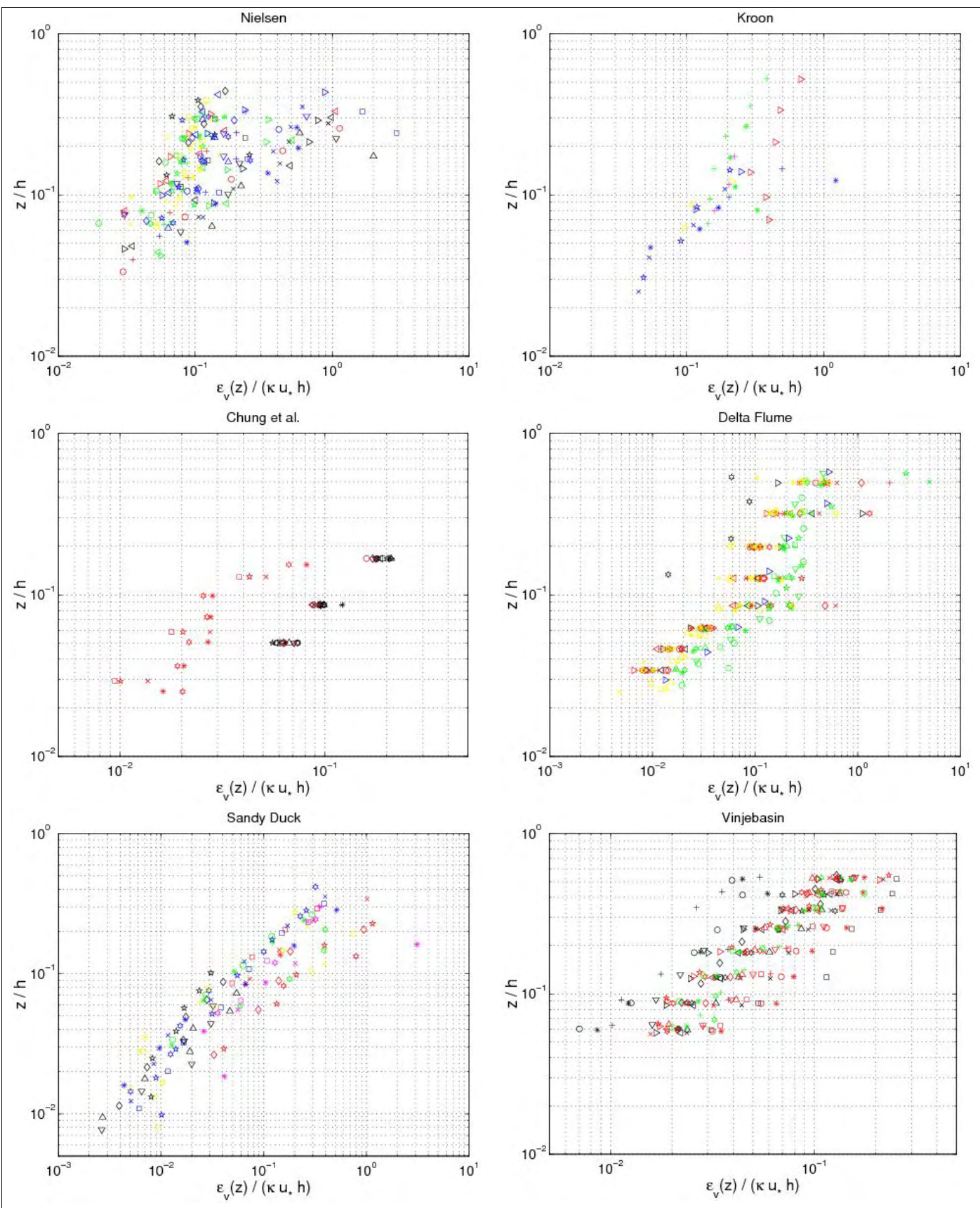

Figure 50. Vertical profile of eddy diffusivity obtained from Equation 130 using measured concentration profiles with interaction between nonbreaking waves and current (data from large-scale facilities and field measurements; each symbol corresponds to particular profile).

\section{Starting point for suspended load}

A difficulty in estimating the suspended sediment load is to determine the elevation that separates suspended load and bed load. For the sheet-flow mode, this elevation should be at the top of the sheet-flow layer. DohmenJanssen (1999) defined the top of the sheet-flow layer as the maximum 
concentration where the interaction between the particles cannot be neglected, i.e., for a volume concentration of $c \approx 0.08$. Figure 51 plots the concentration $c$ versus the elevation $z / h$ using the data from Dohmen-J anssen (1999). These data show some of the first measurements of large concentration close to the bottom in the sheet-flow regime (the sediment diffusivity profiles calculated from these sediment concentration profiles are shown in Figure 49). It appears that, in the sheet-flow layer, the concentration drops from $c_{0} \approx 0.4-0.7$ inside the fixed bed to $c_{b}<0.08$ above the sheet flow. A two-phase model in which the granular interactions are described by application of the kinetic theory appeared to be the more accurate model to describe the sediment concentration profile inside the sheet-flow layer (J enkins and Hanes 1998; Dohmen-J anssen and Hanes 2002).

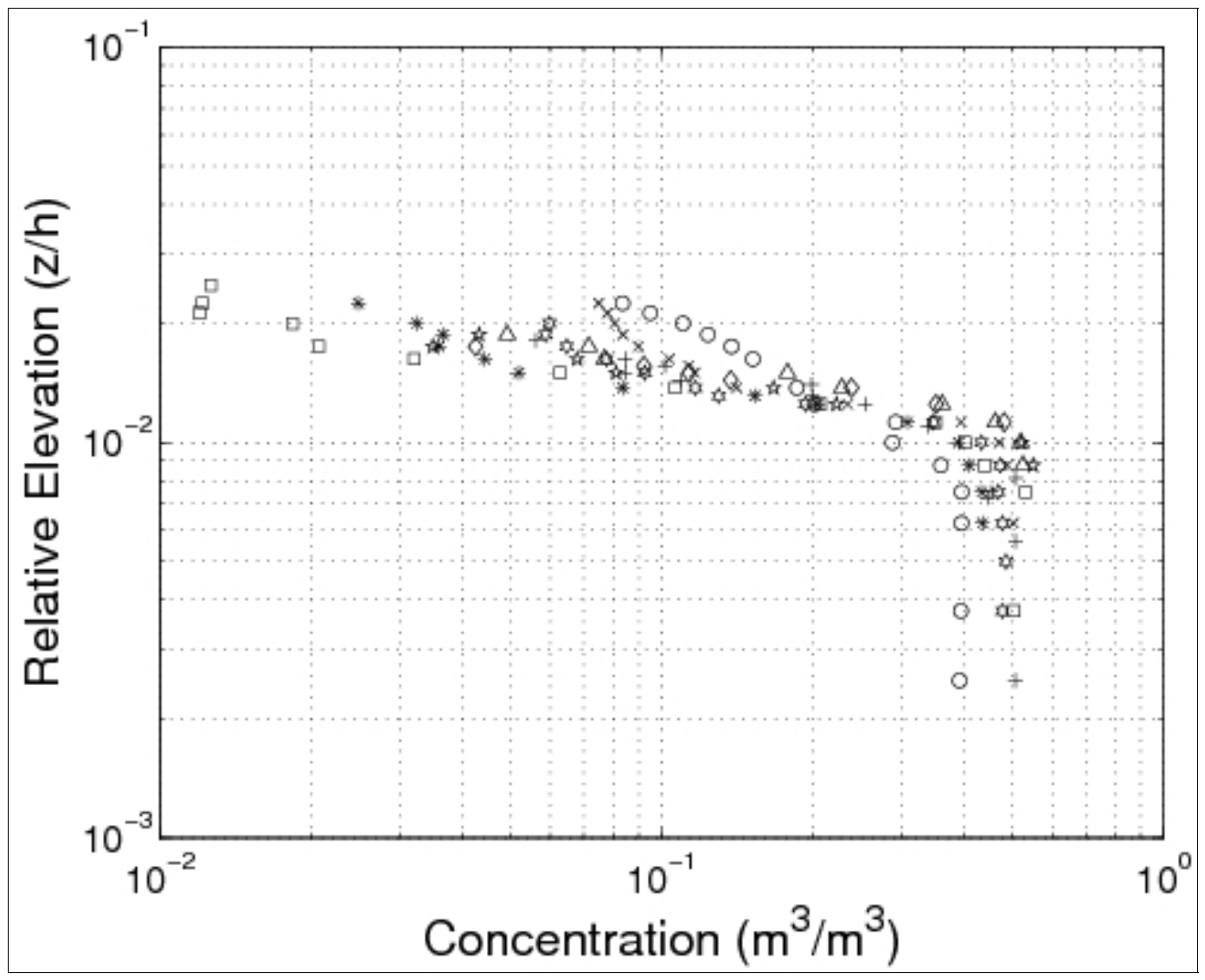

Figure 51. High sediment concentration close to bottom using data from Dohmen-Janssen (1999) (elevation $z$ was increased by $1 \mathrm{~cm}$ to allow for logarithmic representation). 
A simplified formula was developed to estimate a characteristic sediment concentration profile assuming a maximum value $c=c_{0}$ for the fixed bed and a distribution of the sediment diffusivity given by Equation 142 for the suspended sediment. The concentration profile is obtained by applying the advection-diffusion equation taking into account the hindered fall velocity (Equation 10). It can be observed (Figure 52(a)) that such a simple formula produces realistic sediment concentration profiles over the depth.

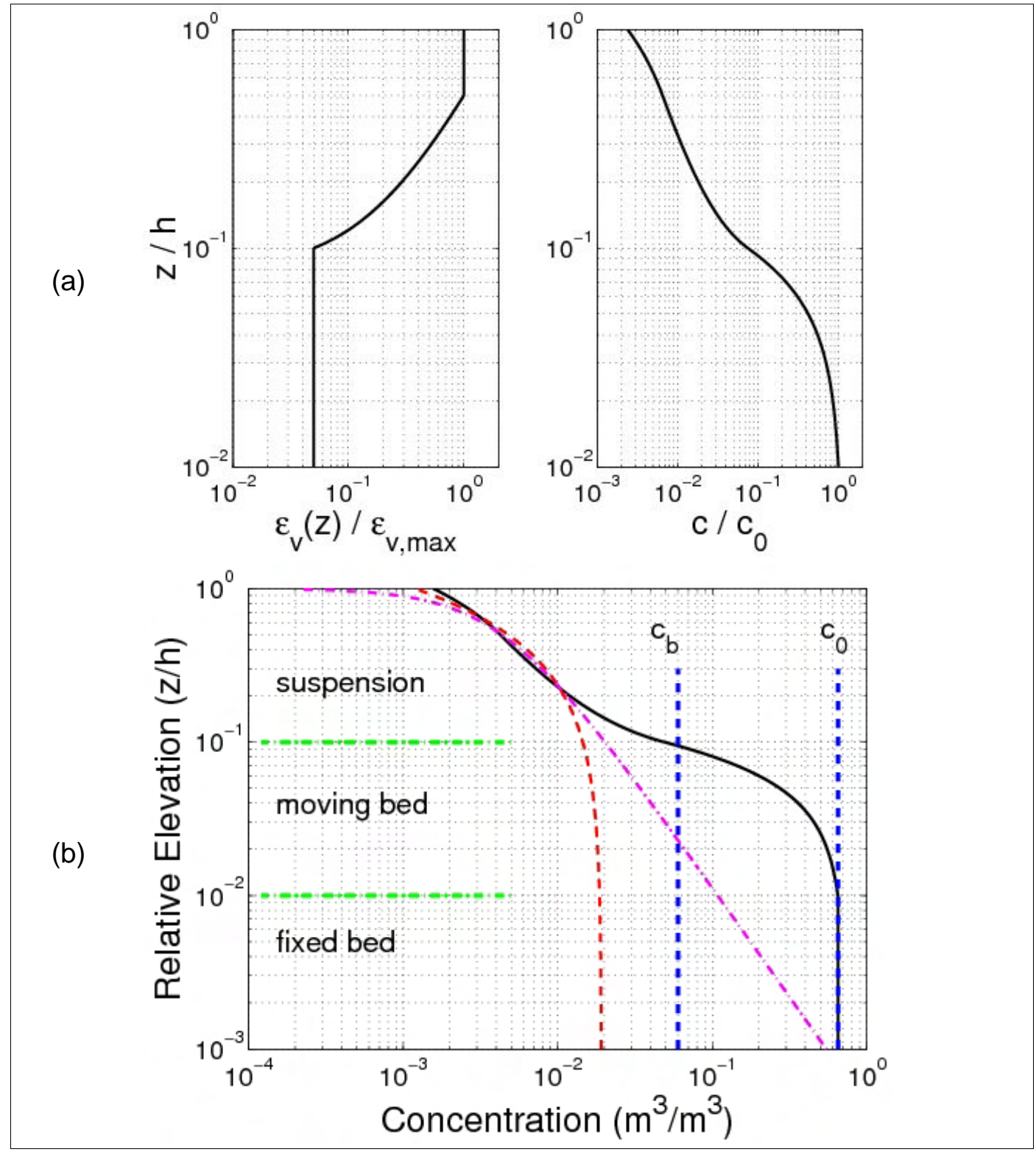

Figure 52. (a) Characteristic sediment diffusion profile (Equation 142) and induced sediment concentration profile, and (b) division of induced concentration profile to different layers and application of an exponential and parabolic logarithmic profile to estimate suspended load. 
For this calculation, $\varepsilon_{w, \max }=510^{-3} \mathrm{~m}^{2} / \mathrm{sec}, \varepsilon_{w, \text { bed }}=110^{-4} \mathrm{~m}^{2} / \mathrm{sec}, z_{w}=0.5 h$, $\delta_{m}=0.1 \mathrm{~h}$ (in Equation 142) and the coefficient $n=1$ were chosen to allow for a correct decrease in concentration in the sheet-flow layer. The bed concentration $c_{b}$ defined at the top of the moving mixing layer $\left(\delta_{m}\right.$ is here assumed to be equal to the sheet-flow layer $\delta_{s}$ ) is then found to be close to the value proposed by Dohmen-J assen and Hanes (2002), i.e., $c_{b} \approx 0.06$ instead of 0.08 , but its value is sensitive to the coefficient $n$ specifying the hindered settling velocity.

Figure 52(b) plots the same concentration profile as in Figure 52(a) represented emphasizing the three different layers where the bed is fixed and moves with large concentration (sheet flow), where suspension occurs. Thus, it is seen that estimation of the suspended concentration profile may be done with simple formulas. A fitted exponential profile and fitted parabolic logarithmic profile were added. It appears that both profiles can yield a correct estimate of the suspended sediment concentration profile using a reference concentration smaller than $c_{b}$. On the other hand, these formulas exhibit limitations close to the bed and thus appear to be sensitive to choice of the reference concentration.

As Van Rijn (1993) found, similar sediment diffusivity profiles were observed for a rippled bed. The thickness $\delta_{m}$ is then assumed to be on the order of the ripple height instead of the thickness of the sheet-flow layer. The same kind of suspended sediment profile should be obtained, whereas in the moving mixing layer, larger and smaller concentration should be observed on the crest and the trough of a ripple, respectively (Figure 53). The sediment diffusivity due to the ripples is large close to the bed because of ripple-generated eddies, but does not increase steeply over the depth because the eddies dissolve traveling upwards. 


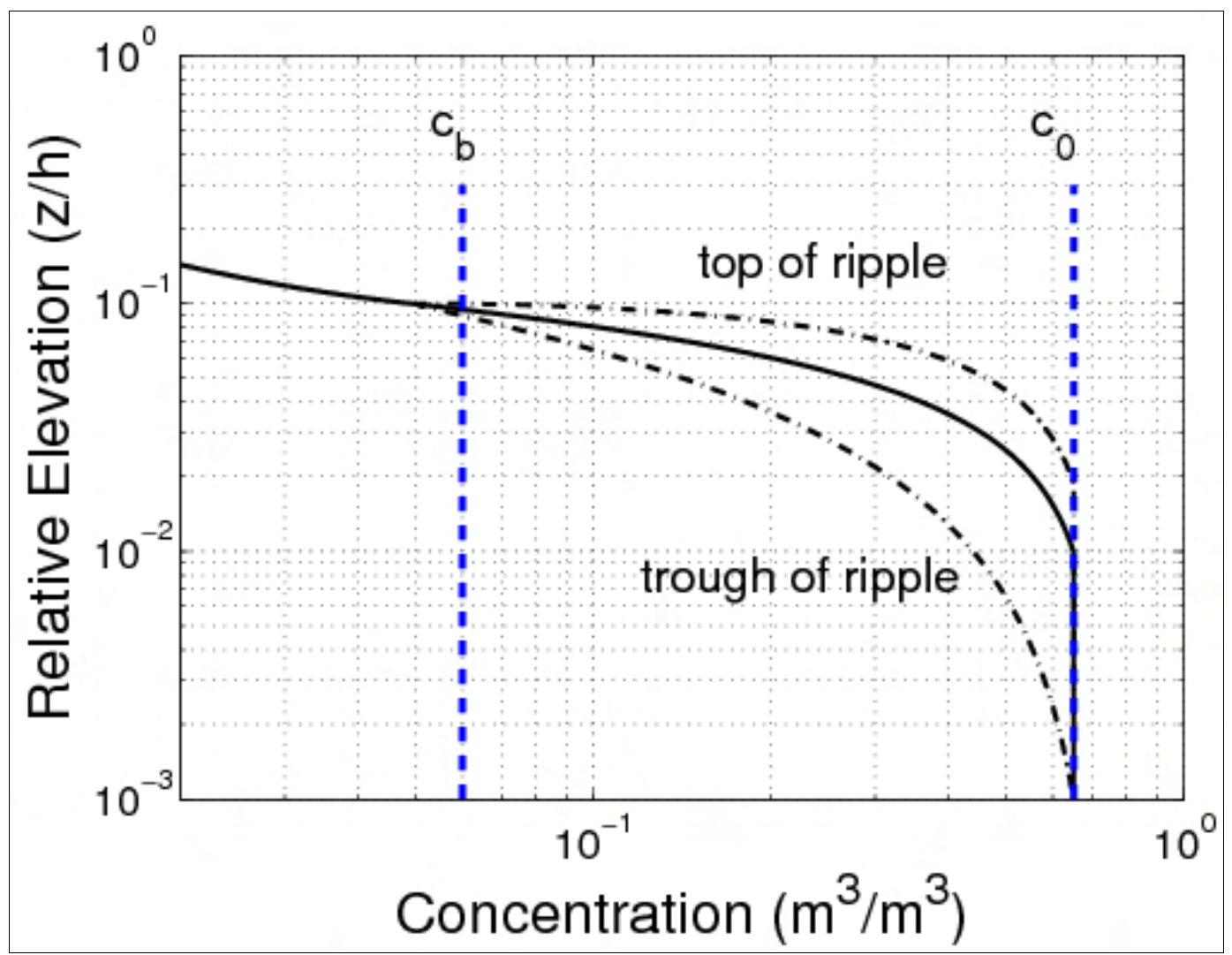

Figure 53. Schematic representation of sediment concentration within moving mixing layer for rippled bed.

\section{Shape of concentration profile}

As for a steady current, concentration profiles described by power-law and exponential equations were fitted to the measurements. For a power-law profile, a linear and a parabolic variation in sediment diffusivity over depth were investigated. Some typical examples of fitted profiles are presented in Figures 54, 55, and 56 for each of the data sets. Table 20 lists the percentage of sediment concentration predicted within a range of 20 percent error for the different data sets presented (pred 20, meas, pred $_{20, \text { powL, }}$, and pred $_{20, p o w}$ denote the fitting of the exponential-law and the linear and parabolic power-laws, respectively). These calculations were also performed for sediment concentration data taken at locations closer to the bed $(z<h / 10)$ or in the upper layer $(z>h / 10)$, where the velocity is approximately constant. 

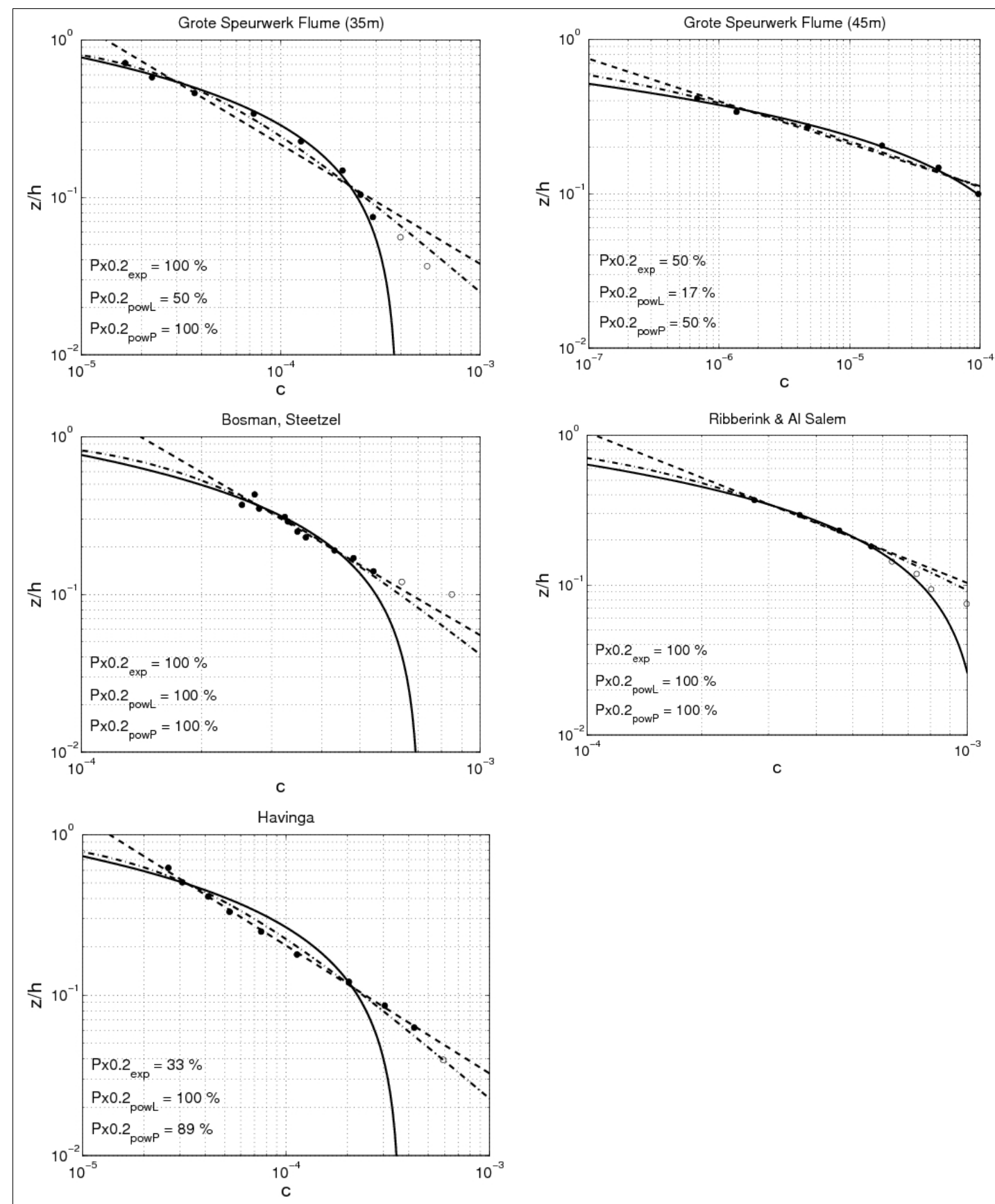

Figure 54. Examples of comparison between predicted concentration using fitted exponential profile (solid line) and power-law profiles (dashed and dashed-dotted line) and measured concentration for interaction between nonbreaking waves and current (data from wave flumes, water tunnels, and basins). 

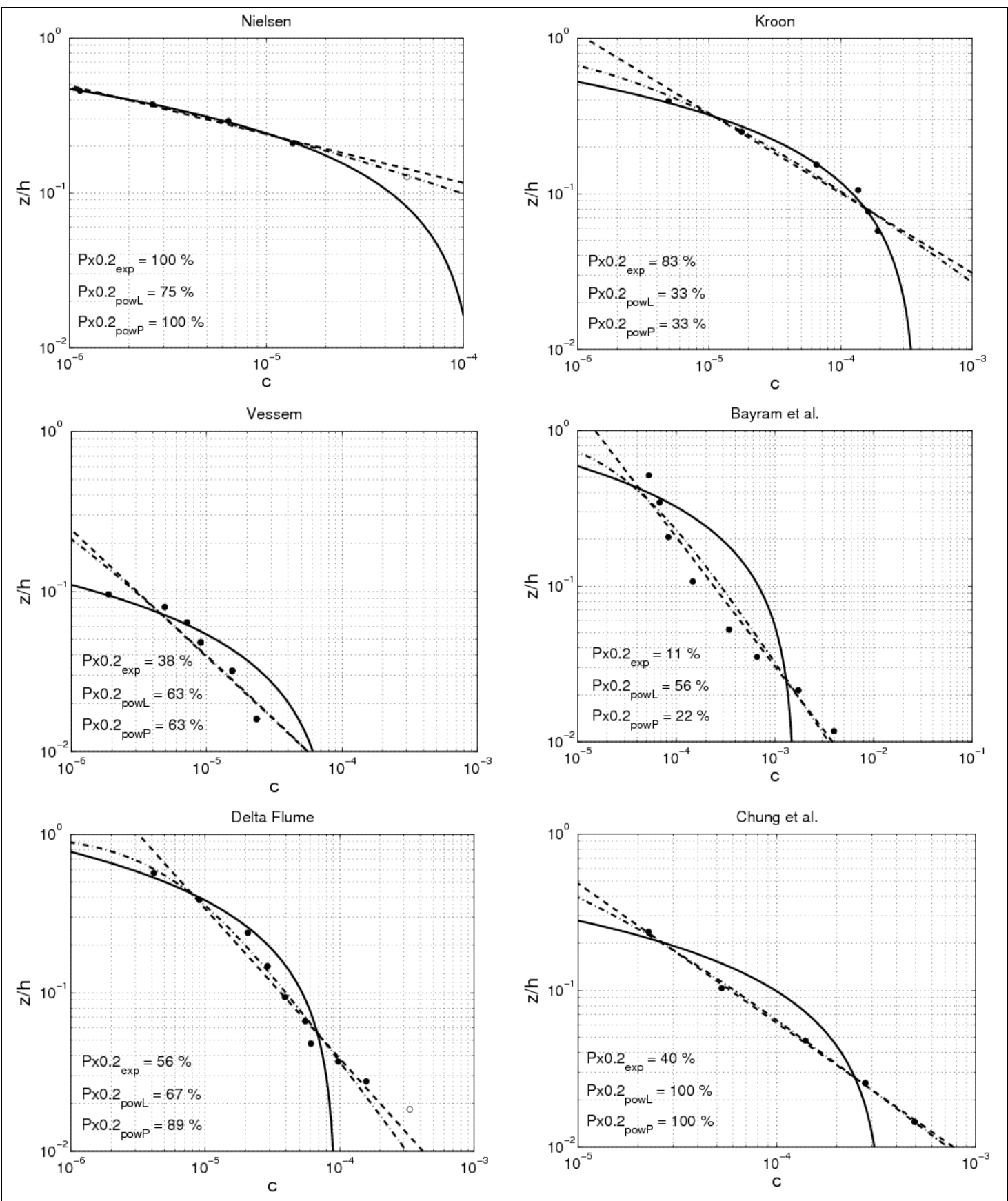

Figure 55. Examples of comparison between predicted concentration using fitted exponential profile (solid line) and power-law profiles (dashed and dashed-dotted line) and measured concentration for interaction between nonbreaking waves and current (data from large-scale facilities and field measurements). 


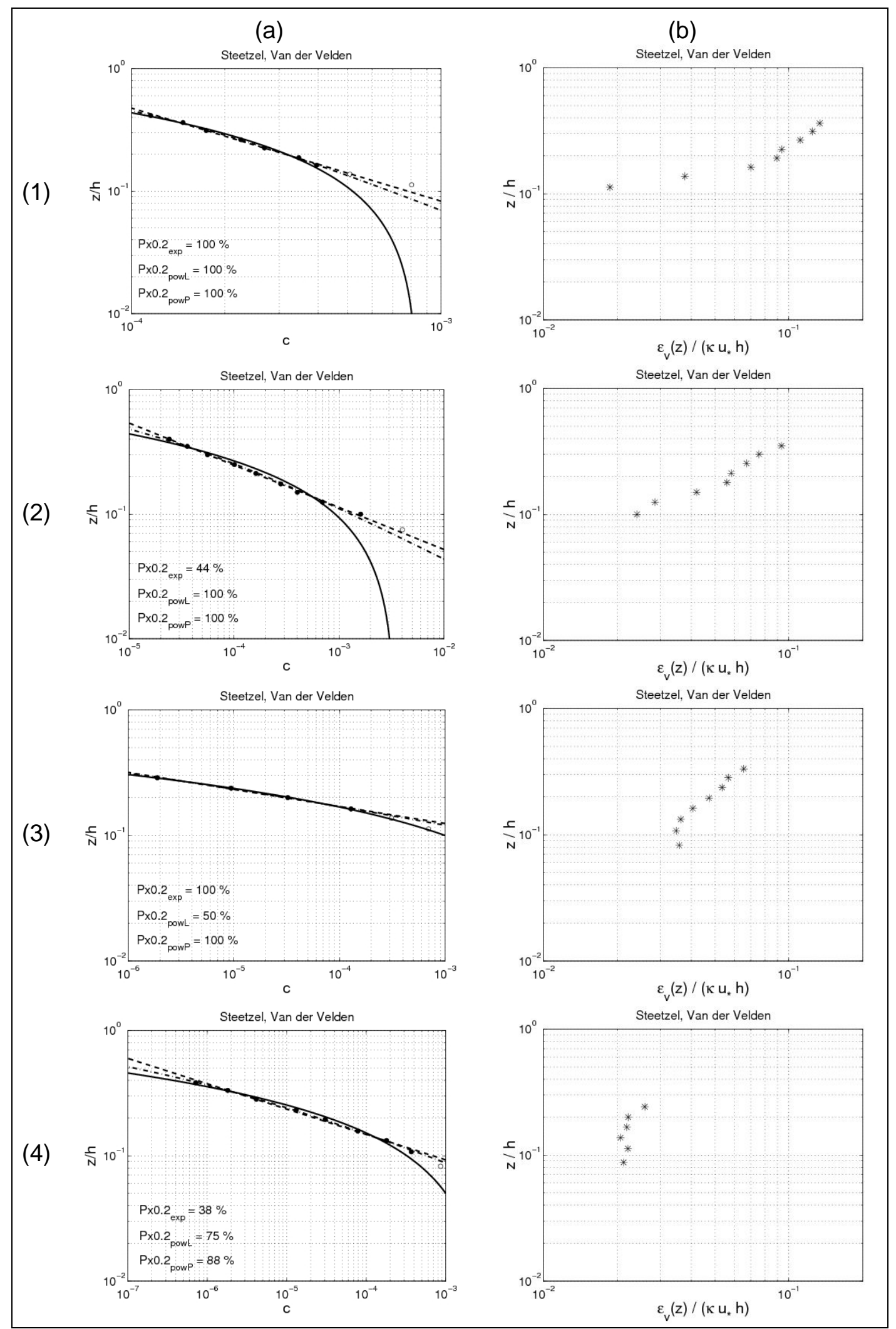

Figure 56. Examples of (a) concentration profiles, and (b) corresponding sediment diffusivity profiles, using data from Steetzel (1984) and Van der Velden (1986). 
Table 20. Percentage of predicted sediment concentrations within $+/-20$ percent of measured values using exponential-law or power-law (linear and parabolic profiles for $\varepsilon_{w}$ ) profiles for studied data sets.

\begin{tabular}{|l|l|l|l|l|l|l|}
\hline \multirow{2}{*}{ Author(s) } & \multicolumn{2}{|c|}{ Exponential (\%) } & \multicolumn{2}{c|}{ Linear Power (\%) } & \multicolumn{2}{c|}{$\begin{array}{c}\text { Parabolic Power } \\
\text { (\%) }\end{array}$} \\
\cline { 2 - 8 } & $z$ & $z<h / 10$ & $z$ & $z<h / 10$ & $z$ & $z<h / 10$ \\
\hline Bosman, Steetzel & 30.5 & 4.6 & 57.9 & 14.3 & 46.5 & 10.0 \\
\hline Nielsen & 35.8 & 15.3 & 54.7 & 23.0 & 53.6 & 22.1 \\
\hline Steetzel, Van der Velden & 62.5 & 15.0 & 69.2 & 14.4 & 72.5 & 15.0 \\
\hline Kroon & 66.3 & 38.9 & 75.0 & 48.1 & 80.0 & 50.0 \\
\hline Havinga & 54.0 & 24.9 & 94.9 & 44.1 & 88.8 & 44.6 \\
\hline Ribberink and Al Salem & 57.6 & 30.4 & 78.3 & 42.7 & 81.2 & 44.7 \\
\hline Chung et al. & 51.5 & 34.7 & 90.1 & 53.5 & 87.1 & 55.4 \\
\hline Vessem & 50.8 & 41.1 & 69.4 & 53.2 & 69.9 & 54.3 \\
\hline Grote Speurwerk (35 m) & 55.7 & 28.0 & 38.9 & 21.0 & 53.6 & 27.7 \\
\hline Grote Speurwerk (45 m) & 73.9 & 27.5 & 68.3 & 21.8 & 75.9 & 25.0 \\
\hline Deltaflume & 18.4 & 13.8 & 57.0 & 29.7 & 49.1 & 28.0 \\
\hline Bayram et al. & 29.3 & 15.9 & 67.8 & 42.7 & 61.5 & 38.9 \\
\hline Total & 53.2 & 21.7 & 65.8 & 26.9 & 67.2 & 27.7 \\
\hline
\end{tabular}

Fitting of the three theoretical profiles gives poorer agreement with the data (especially for the exponential profile) compared to the steady flow situation. Less than 60 percent of the data are predicted within a factor of 1.2 by an exponential profile, and less than 70 percent by a fitted powerlaw profile. These figures drop to below 30 percent for data close to the bed (where $z / h<0.1$ ). Some explanations may be suggested for the increased discrepancy:

- Several data sets correspond to field experiments where measurements are not as easy to make as in the laboratory, and so the variability in general is larger (Nielsen (1984), "Vessem," and Bayram et al. (2001) data sets).

- Contrary to the steady flow data, it appears for several data sets that two layers occur for suspension. The layer closest to the bed (defined as the "moving mixing layer" by Van Rijn 1989) corresponds to much smaller sediment diffusivity compared to the upper layer, and it may sometimes include bed-load transport. 
- For the specific case of fitting an exponential profile, if two layers appear, fitting to all the data typically presents difficulty over some range of values (Figure 54: Bosman (1982), Steetzel (1985), and Figure 55: “Vessem” (Van Rijn et al. 2001), Bayram et al. (2001), and Delta Flume).

- Few measurements were made close to the mean water surface (generally limited to the trough level of the waves). It is thus difficult to observe the decrease of the sediment diffusivity as was done for a steady current.

Figure 56 plots four different concentration profiles and the sediment diffusivity profiles typically observed using the Steetzel (1985) and Van der Velden (1986) data sets shown (small water-tunnel with $d_{50}=0.22 \mathrm{~mm}, h=0.4 \mathrm{~m}, U_{c}=0 \mathrm{~m} / \mathrm{sec}$ ). The input parameters are listed in Table 21. Some of the experiments correspond to nonphysical waves, because the steepness $\lambda_{w}$ would be greater than 0.14 .

Table 21. Input parameters for four study cases in Figure 56.

\begin{tabular}{|l|l|l|l|l|l|}
\hline Case & $U_{w}(\mathrm{~m} / \mathrm{sec})$ & $T_{w}(\mathrm{sec})$ & $\lambda_{w}$ & $H_{r}(\mathrm{~m})$ & $L_{r}(\mathrm{~m})$ \\
\hline 1 & 0.75 & 1.0 & 0.324 & 0.020 & 0.300 \\
\hline 2 & 0.50 & 1.5 & 0.090 & 0.013 & 0.080 \\
\hline 3 & 0.30 & 1.5 & 0.054 & 0.013 & 0.105 \\
\hline 4 & 0.50 & 2.0 & 0.059 & 0.023 & 0.130 \\
\hline
\end{tabular}

It may be seen in Figure 56 that the concentration measurements inside the moving mixing layer largely influence the profile fitting. The results are affected by the difference between the value of the sediment diffusivity inside the moving mixing layer $\varepsilon_{w, \text { bed }}$ and at $z_{w}$ (maximum value $\varepsilon_{w, \max }$ ). Usually, the difference $z_{w}-\delta_{m}$ is small, and corresponds to a transition zone. Up to $z_{w}$, the sediment diffusivity is a weak function of $z / h$ such that both the exponential and power-law fits yield acceptable results.

- Figure 56(1) represents a situation where no data points were available inside the moving mixing layer; the difference between the sediment diffusivity inside the mixing layer and its maximum value is large $\left(\varepsilon_{w, b e d} / \varepsilon_{w, \max }<0.2\right)$. The two layers can be clearly observed in the concentration profile. A fitted exponential profile induces a large 
underestimation of the concentration when $z / h<0.1$. The orbital wave velocity is large compared to the wave period $\left(\lambda_{w}>0.14\right)$.

- Figure 56(2) represents a situation where some data points were obtained inside the moving mixing layer; the difference between the sediment diffusivity inside the mixing layer and its maximum value is not as large as for Figure 56(1) $\left(\varepsilon_{w, b e d} / \varepsilon_{w, \max } \approx 0.3-0.4\right)$. The two layers cannot be observed in the concentration profile. A fitted exponential profile produces a large underestimation of the concentration for $z / h<$ 0.1 . On the other hand, a power-law profile gives a good approximation of the concentration profile.

- Figure 56(3) represents a situation where some data points were obtained inside the moving mixing layer; the difference between the sediment diffusivity inside the mixing layer and its maximum value is small ( $\left.\varepsilon_{w, b e d} / \varepsilon_{w, \max }>0.4\right)$. The two layers cannot be observed in the concentration profile. The fitted exponential profile seems to yield a good approximation of the concentration profile, and if a power-law profile yields somewhat better results.

- Figure 56(4) represents a situation where the sediment diffusivity is approximately constant over the depth $\left(\varepsilon_{w, b e d} \approx \varepsilon_{w, \max }\right)$. A fitted exponential profile gives the best representation of the concentration profile.

It appears that the ratio $\varepsilon_{w, \max } / \varepsilon_{w b e d}$ (and more specifically $\varepsilon_{w, \max }$ ) is proportional to the wave orbital velocity $U_{w}$ and inversely proportional to the wave period $T_{w}$, which means proportional to the wave steepness $\lambda_{w}$. For realistic waves where $\lambda_{w}<0.14$, the exponential profile yields an accurate approximation of the sediment concentration profile.

Similar observations were made for the Bosman (1982) and Steetzel (1984), "Grote Speurwerk (35 m), Vessem, Delta Flume” (Van Rijn et al. 2001), and Ribberink and Al Salem (1994) data sets.

Following these results and what was obtained for a mean current, only the mean sediment diffusivity over the water depth (exponential concentration profile) is examined in the following.

\section{Relationships for mean sediment diffusivity under waves}

Because the shear stress cannot be calculated from measurements, but must be estimated, large uncertainties appear in the calculation of the 
ratio $\varepsilon_{\mathbf{w}} /\left(\kappa h u^{*} w\right)$, especially because of the influence of bed forms. Indeed, for most of the cases with low and mid-energy wave dissipation, ripples occur and strongly influence the suspended sediment profile. Furthermore, in most of the previous studies, semi-empirical relationships proposed for $\varepsilon_{w}$ were typically not based on the orbital motion due to waves.

Dally and Dean (1984) assumed that the classical expression given by Rouse (1938) may be applicable, assuming a parabolic profile and using its mean value over the depth (see "Sediment diffusivity due to steady current" section):

$$
\varepsilon_{w, E}=\frac{1}{6} \kappa u_{* w} h
$$

Skafel and Krishnappan (1984) employed a simple model to estimate the suspended sediment distribution assuming an exponential sediment suspension profile. The mean sediment diffusion $\varepsilon_{w}$ was evaluated by the following expression:

$$
\varepsilon_{w, E}=\beta_{w} A_{w} u_{*}
$$

where $\beta_{w}$ is a dimensionless diffusion coefficient ( $\beta_{w} \approx 0.1$ ). Using their own data set (small wave flume with glass beads, $s=2.5$ and $d_{50}$ $=0.15 \mathrm{~mm}$ ), and assuming $k_{s}=H_{r}$ for the calculation of $u{ }^{*} w$, they found the following relationship for $\beta_{w}$ :

$$
\beta_{w}=8.7\left(\frac{d_{50} u_{*}}{v}\right)^{-2.2}
$$

Van Rijn (1989) proposed empirical expressions for the minimum and maximum values of the sediment diffusivity (Equation 142):

$$
\begin{aligned}
\varepsilon_{w, \text { bed }} & =0.004 d_{*} U_{w} \delta_{m} \\
\varepsilon_{w, \max } & =0.035 \frac{H_{s} h}{T_{p}}
\end{aligned}
$$

where $H_{s}$ and $T_{p}$ are the significant wave height and the peak wave period, respectively. 
Kosyan (1985) developed a semi-empirical model to estimate the diffusion coefficient distribution over the water depth. It appeared that the main component is produced by the orbital motion, and the distribution was found to be, using small-amplitude wave theory,

$$
\varepsilon_{w}=\frac{\pi H_{w}^{2}}{2 \sqrt{2} T_{w}} \frac{\sinh ^{2} k z}{\sinh ^{2} k h}
$$

where $H_{w}$ is the wave height. The mean value over the depth is:

$$
\varepsilon_{e, E}=\frac{\pi H_{w}^{2} h}{4 \sqrt{2} T_{w}}\left(\frac{\sinh 2 k h}{2 k h}-1\right) \approx \frac{8 \pi^{3} U_{w}^{3} h^{3}}{3 \sqrt{2} g^{2} T_{w}^{3}}
$$

For waves only and a rippled bed, Nielsen (1992, pp. 215-217) observed that an exponential profile describes the concentration profile well. He found that the mean sediment diffusivity is closely related to the ripple height $H_{r}$ for sharp-crested ripples and suggested:

$$
\begin{aligned}
\varepsilon_{w, E} & =0.075 U_{w} H_{r} \text { for } \frac{U_{w}}{W_{s}} \leq 18 \\
& =1.4 W_{s} H_{r} \text { for } \frac{U_{w}}{W_{s}}<18
\end{aligned}
$$

Analyzing the data set previously presented (Table 19), where waves are not breaking and the mean current is negligible $\left(\left|U_{c}\right|<0.05\right)$, a comparison was made between the different formulas studied and the data. The percentage of predicted values within a factor of 1.5, 2, and 5 deviation (denoted by $P x 1.5, P x 2$, and $P x 5$, respectively) as well as the mean value and the standard deviation of the ratio $f\left(\varepsilon_{w, E}\right)=\log \left(\varepsilon_{w, \text { pred }} / \varepsilon_{w, \text { meas }}\right)$ are presented in Table 22 .

Of the studied formulas, the one proposed by Dally and Dean (1984), who assumed that the Rouse expression could be used, gives the least scatter, even if the formula in general overestimates the diffusivity. This expression may be correct, but the Schmidt number appears to be much smaller than 1 . The more complex formulas introduced by Skafel and Krishnappan (1984) and Nielsen (1992, pp. 215-217) yield better overall fit (especially the Nielsen formula), but also larger scatter. 
Table 22. Predictive skill of different formulas for sediment diffusivity for waves only.

\begin{tabular}{|l|l|l|c|c|l|}
\hline Author(s) & $P \times 1.5(\%)$ & $P \times 2(\%)$ & $P \times 5(\%)$ & $\operatorname{Mean}\left(f\left(c_{R}\right)\right)$ & $\operatorname{Std}\left(f\left(c_{R}\right)\right)$ \\
\hline Dally and Dean & 3 & 10 & 51 & 0.69 & 0.29 \\
\hline Skafel and Krishnappan & 3 & 7 & 35 & -0.68 & 0.76 \\
\hline Kos'yan & 12 & 17 & 49 & -0.35 & 0.94 \\
\hline Van Rijn & 23 & 38 & 76 & 0.29 & 0.52 \\
\hline Nielsen & 33 & 60 & 89 & -0.24 & 0.38 \\
\hline Equation 152 & 58 & 86 & 99 & -0.02 & 0.22 \\
\hline Equation 153 & 46 & 68 & 98 & 0.09 & 0.29 \\
\hline Equation 154 & 45 & 69 & 100 & 0.09 & 0.28 \\
\hline
\end{tabular}

\section{New formula for mean sediment diffusivity due to waves}

Assuming that the Rouse expression can be adopted, the ratio $\varepsilon_{w, E} /(\kappa h u * w)$ was plotted against the main parameters. As observed by Nielsen (1992), this ratio is related to $U_{w} / W_{s}$ and to wave period (Figure 57). Based on the studied data set, a new empirical equation is proposed:

$$
\varepsilon_{w, E}=410^{-3}\left(\frac{g^{2 / 3} T_{w}}{v^{1 / 3}}\right)^{0.5}\left(\frac{U_{w}}{W_{s}}\right)^{-0.5} \kappa u_{*} h
$$

Equation 152 presents the best results among the studied formulas. More than 85 percent of the data points are correctly predicted within a factor 2 and nearly 100 percent within a factor 5 . Also, Figure 58 shows that the results do not depend on the data set.

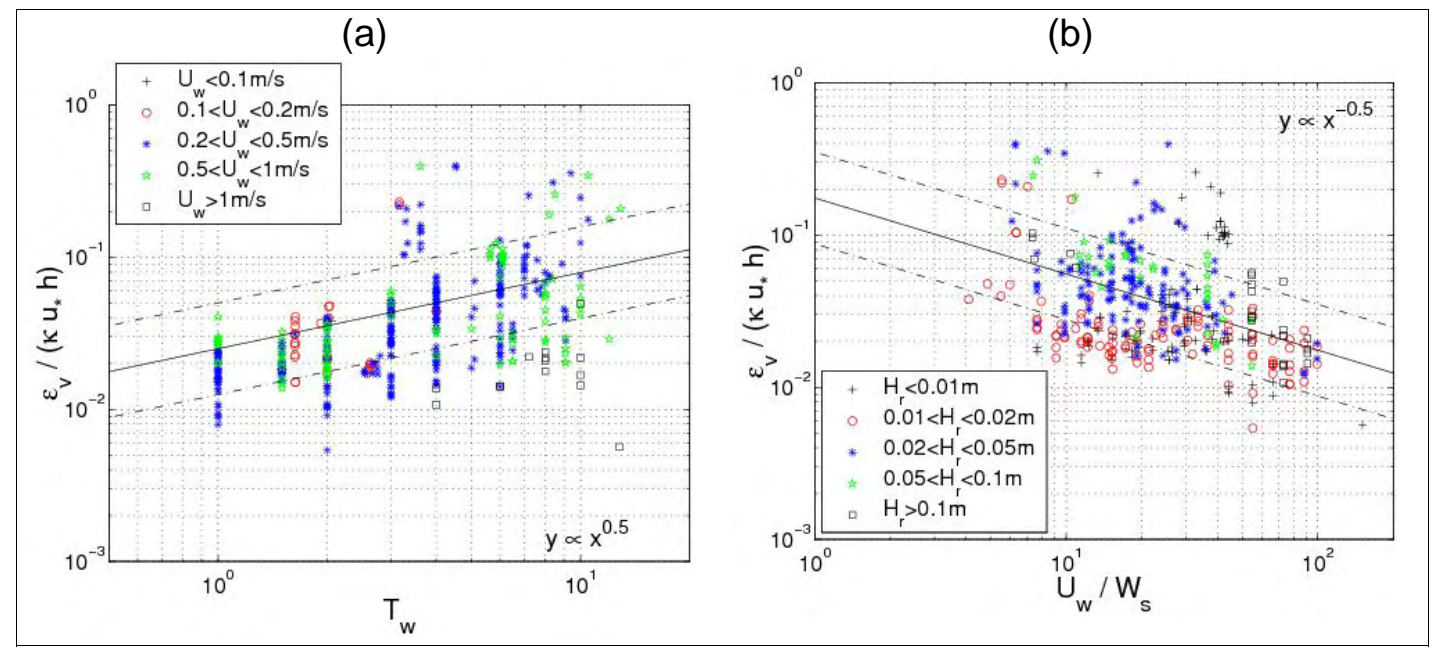

Figure 57. Dimensionless sediment diffusivity $\varepsilon_{w, E} /\left(\kappa h u \star_{w}\right)$ versus (a) wave period, and (b) ratio $U_{w} / W_{s}$. 


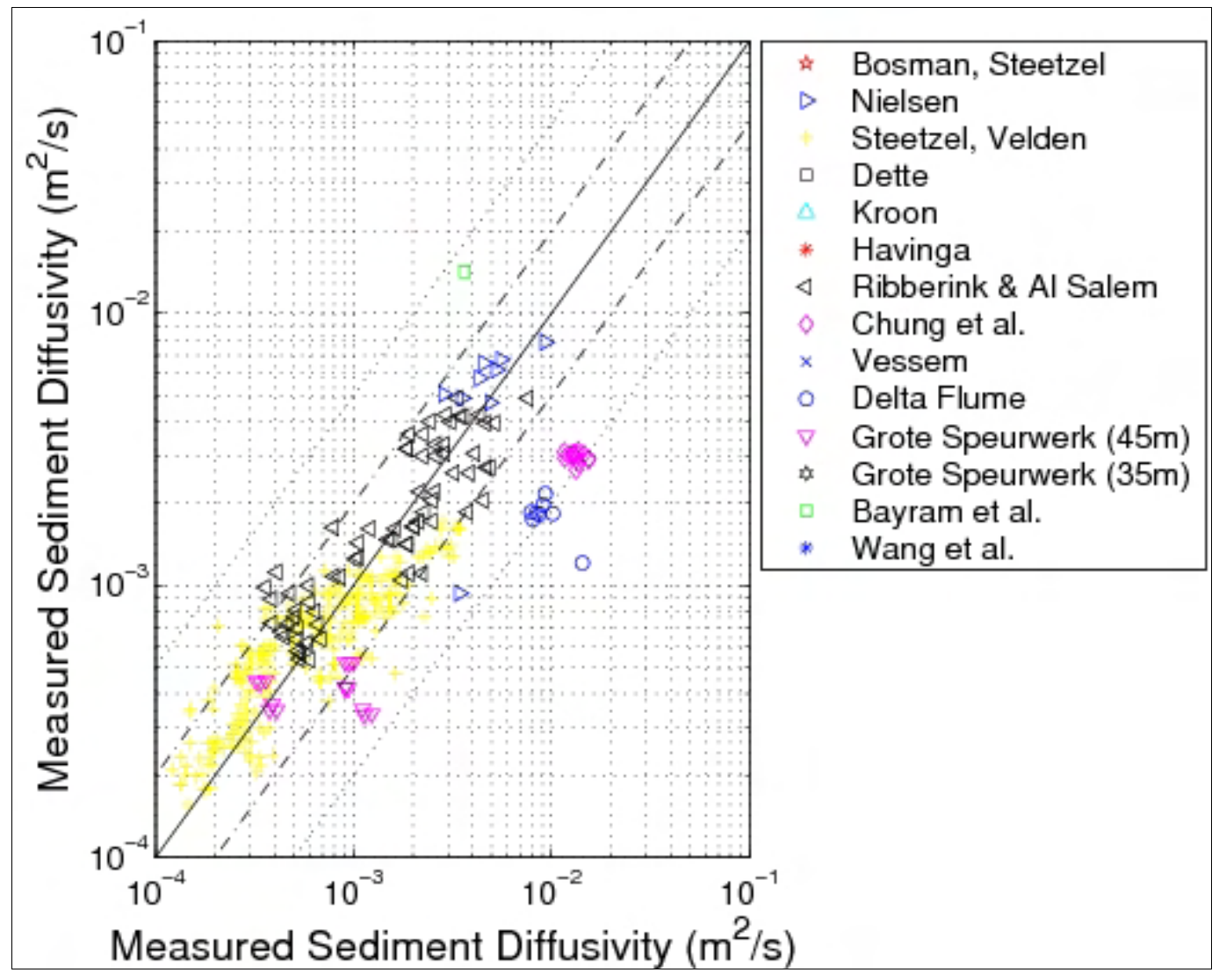

Figure 58. Vertical sediment diffusivity $\varepsilon_{W, E}$ estimated from data compiled versus $\varepsilon_{W, E}$ calculated with Equation $152\left(U_{c}<0.05\right)$.

However, following the study performed for the sediment diffusivity due to a current ("Sediment diffusivity under steady current" section), the correction factor (Schmidt number) may be taken to be a function of the ratio $W_{s} / u^{*} w$. The sediment diffusivity under waves may be written as an average over the wave period for an instantaneous sediment diffusivity, which for an exponential profile leads to $\varepsilon_{w, E}=\sigma_{w} \kappa u_{*} h /(3 \pi)$, where $\sigma_{w}$ is the wave-related Schmidt number, and the coefficient $2 / \pi$ corresponds to the time average of the function $|\sin |$.

Plotting the dimensionless sediment diffusivity $\varepsilon_{w, E} /\left(\kappa h u^{*} w\right)$ versus the ratio $W_{s} / u_{*}$ (Figure 59), a trend appears similar to that observed for a current (Figure 48). Using the previous data sets, an expression for $\varepsilon_{w, E}$ is proposed following the equation developed by Van Rijn (1984b) (Equation 122), which yields the following expression for the Schmidt number: 


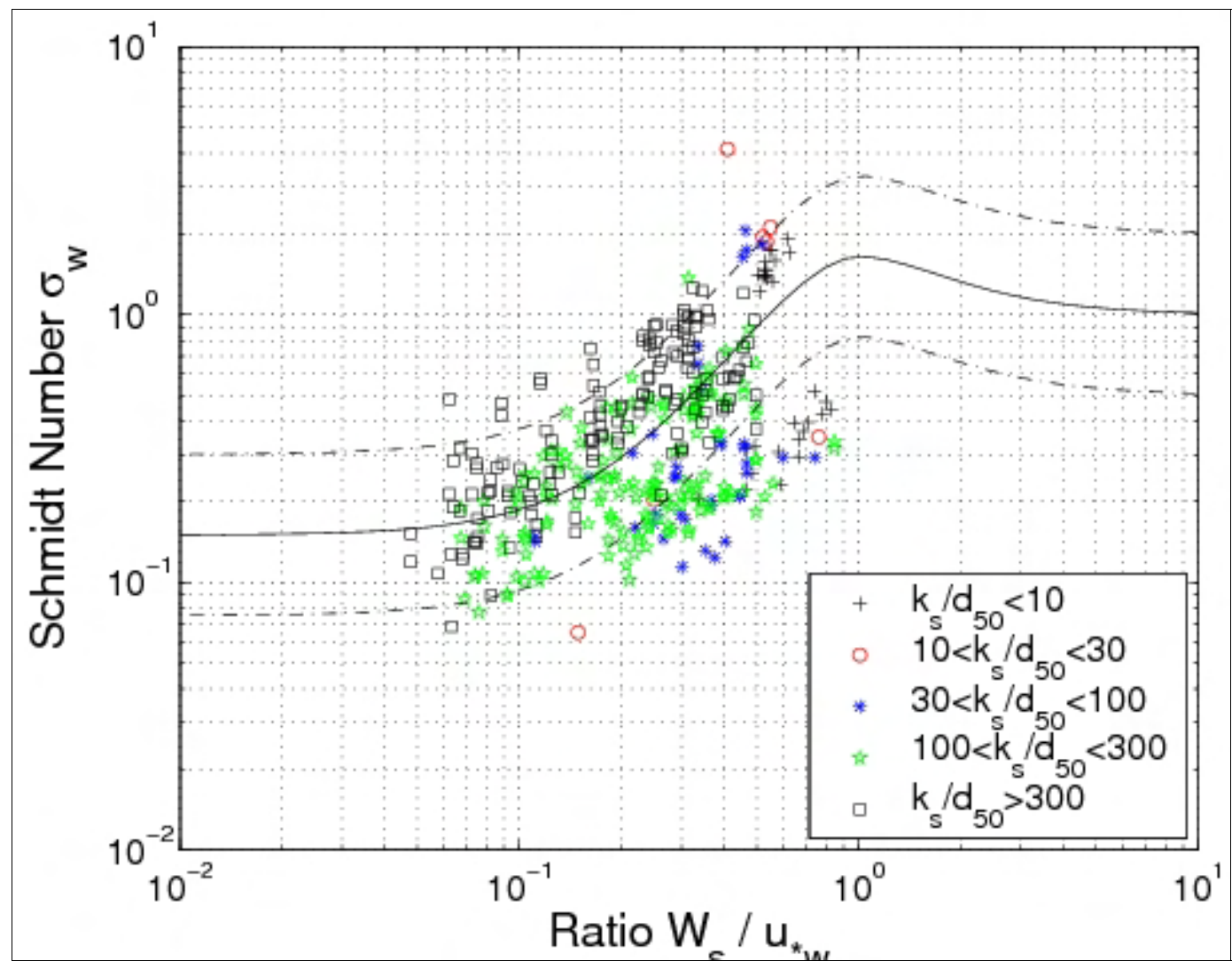

Figure 59. Estimated value of coefficient $\sigma_{w}$ using Equation 154 as function of ratio $W_{s} / u_{*}$ with roughness ratio $k_{s} / d_{50}$ indicated.

$$
\sigma_{w}=0.2+2.5\left(\frac{W_{s}}{u_{* w}}\right)^{2}
$$

To put forward a relationship that gives physically meaningful results for all conditions, it should be considered that if the ratio $W_{s} / u_{* w} \gg 1$, the Schmidt number should be equal to 1 . Thus, a similar expression is proposed as for the steady current regarding the sediment diffusivity (Equation 136 and Figure 59):

$$
\sigma_{w}= \begin{cases}0.15+1.5 \sin ^{2}\left(\frac{\pi}{2} \frac{W_{s}}{u_{*_{w}}}\right) & \text { if } \frac{W_{s}}{u_{*_{w}}} \leq 1 \\ 1.0+0.65 \sin ^{2}\left(\frac{\pi}{2} \frac{u_{*_{w}}}{W_{s}}\right) & \text { if } \frac{W_{s}}{u_{*_{w}}}>1\end{cases}
$$


The wave-induced Schmidt number (Equations 153 and 154) is often considerably smaller than that found for steady current (factor 2 or 3 smaller). However, because the friction velocity for waves is generally much larger than for a current, the mixing from waves is also much larger. As discussed, this mixing may decrease the Schmidt number. The oscillatory velocities at the bottom may also affect the Schmidt number, and the results obtained using these formulas are not as good as the results obtained using Equation 152. An improvement, however, is obtained in agreement with the data compared to the other studied formulas: 45 percent of the data are correctly predicted with a permitted error of a factor 1.5, and 69 percent with a permitted error of a factor 2 .

There seems to be a relationship between $\sigma_{w}$ and the roughness ratio $k_{s} / d_{50}$ (Figure 59). Because this roughness ratio (and the total shear stress) is calculated using empirical formulas and not estimated directly from the measurements (contrary to the data for current only), the relationship between $\sigma_{w}$ and $k_{s} / d_{50}$ exhibits larger scatter. Another explanation could be that the proposed Schmidt number does not take into account a possible effect of wave period as observed in Equation 152.

\section{Interaction between waves and current}

If waves and current interact, the most straight-forward approach would be to add the two values obtained from current only (Equations 134 and 136) and waves only (Equation 154), i.e., $\varepsilon_{c w, E}=\varepsilon_{w, E}+\varepsilon_{c, E}$. However, it may be noted that this summation does not present correct results compared to the measurements (Figure 60). Indeed, the larger the ratio $U_{c} / U_{w}$ is, the larger the overestimation of the measurements. On the other hand, using the sediment diffusivity for waves only produces a large underestimation (see Table 23). The overestimation obtained using a summation approach may also be attributed to the calculation of the total shear velocity including the current. Indeed, for the calculation of $u^{*}$, the roughness was assumed to be equal to the wave-induced Nikuradse roughness (a function of the wave-induced ripples), even if the direction of the current may affect this value. 


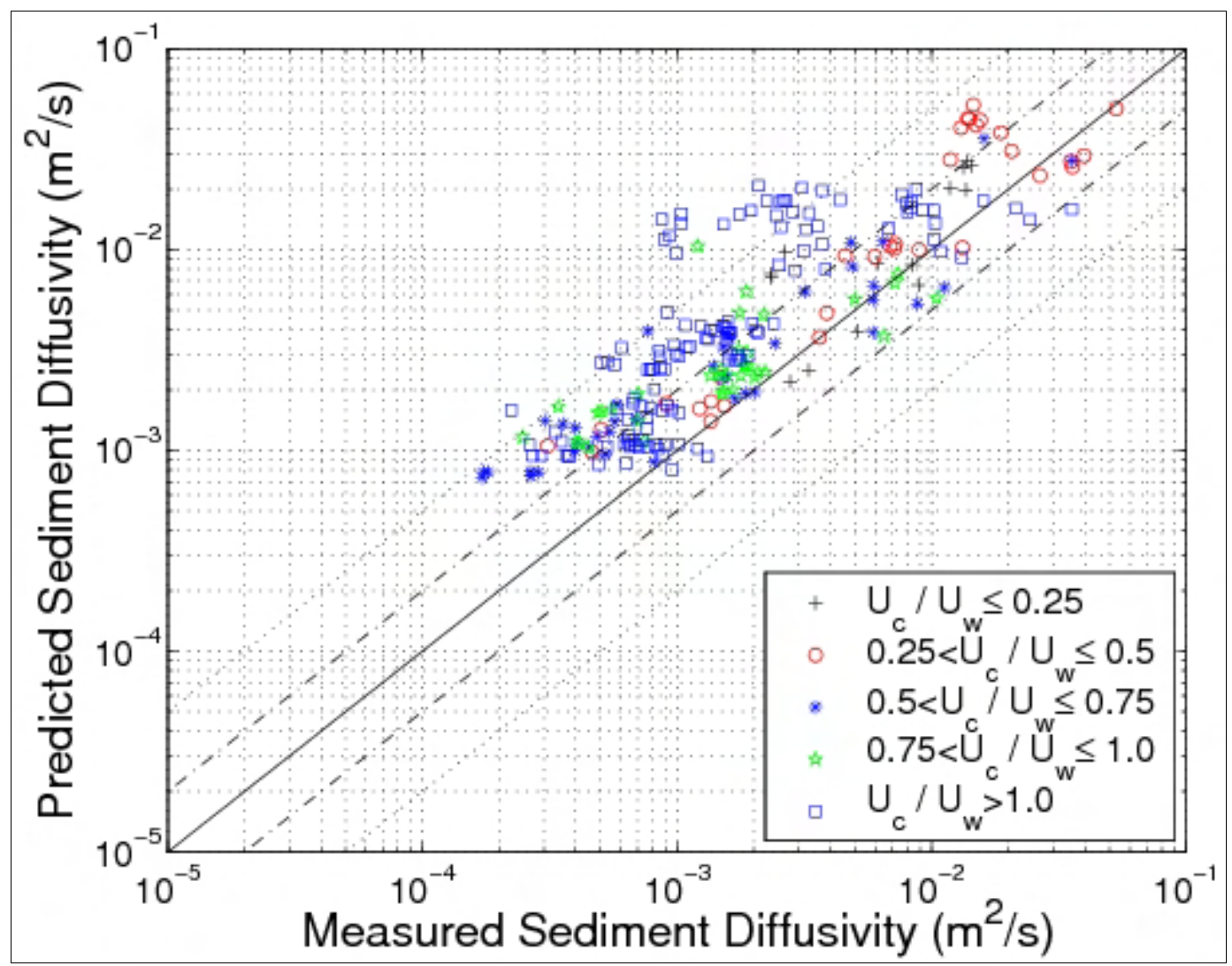

Figure 60. Estimation of sediment diffusivity $\varepsilon_{c w}$ by adding current- and wave-related sediment diffusivity as function of parameter $W_{s} / u_{*_{w}}$ with ratio $U_{c} / U_{w}$ emphasized.

Table 23. Prediction of sediment diffusivity for wave and current interaction (for different calculations of $\varepsilon_{c w, E}$, Equations 136 and 154 are used for obtaining $\varepsilon_{c, E}$ and $\varepsilon_{w, E}$, respectively).

\begin{tabular}{|l|l|l|l|l|}
\hline Equation & $P \times 2(\%)$ & $P \times 5(\%)$ & Mean $\left(f\left(c_{R}\right)\right)$ & $\operatorname{Std}\left(f\left(c_{R}\right)\right)$ \\
\hline Dally and Dean (1984) & 55 & 89 & 0.20 & 0.39 \\
\hline Van Rijn (1989) & 37 & 83 & 0.18 & 0.50 \\
\hline Nielsen (1992) & 0 & 1 & -1.68 & 0.80 \\
\hline$\varepsilon_{c w, E}=\varepsilon_{c, E}+\varepsilon_{w, E}$ & 51 & 86 & 0.29 & 0.36 \\
\hline$\varepsilon_{c w, E}=\varepsilon_{w, E}$ & 49 & 94 & -0.22 & 0.34 \\
\hline$\varepsilon_{c w, E}=\sqrt{\varepsilon_{c, E}^{2}+\varepsilon_{w, E}^{2}}$ & 58 & 88 & 0.18 & 0.38 \\
\hline$\varepsilon_{c w, E}=X_{v} \varepsilon_{c, E}+\left(1-X_{v}\right) \varepsilon_{w, E}$ & 60 & 91 & 0.02 & 0.40 \\
\hline $\begin{array}{l}\varepsilon_{c w, E}=\varepsilon_{c, E}+\varepsilon_{w, E} \text { using } \\
\sigma_{c}=\sigma_{w}=\sigma_{c w}(\text { Equation 156) }\end{array}$ & 65 & 92 & 0.09 & 0.38 \\
\hline
\end{tabular}


Van Rijn (1989) proposed use of a mean quadratic value of the currentrelated and wave-related sediment diffusivity:

$$
\varepsilon_{c w}=\sqrt{\varepsilon_{c}^{2}+\varepsilon_{w}^{2}}
$$

Thus, a correction factor may be applied to compute the diffusivity for waves and current combined. Using the approach of Grant and Madsen (1979) for the computation of wave-current friction factors, i.e., $\varepsilon_{c w, E}=$ (1- $\left.X_{v}\right) \varepsilon_{w, E}+X_{v} \varepsilon_{c, E}$ with $X_{v}=\left|U_{c}\right| /\left(\left|U_{c}\right|+U_{w}\right)$, improved results are expected (Table 23). However, it seems that the effect of the current on the sediment diffusivity is suppressed by the waves. Indeed, the Grant and Madsen (1979) method presents good results mainly if $U_{c} / U_{w}>0.5$. For $U_{c} / U_{w}<0.5$, it tends to underestimate $\varepsilon_{c w, E}$.

This overestimation may be attributable to the Schmidt number perhaps being the same for a current and for waves. A more physical description of the wave and current interaction should be based on a unique Schmidt number, calculated as an empirical weighted value between $\sigma_{c}$ and $\sigma_{w}$. According to the data, the effect of the waves seems to be larger, and the following relationship is thus proposed:

$$
\sigma_{c w}=Y \sigma_{c}+(1-Y) \sigma_{w}
$$

where $Y=\theta_{c} /\left(\theta_{c}+\theta_{w}\right)$. The scatter may be due to uncertainties in the estimation of the current- and wave-induced shear stress.

In Figure 61, the vertical sediment diffusivity $\varepsilon_{c w, E}$ estimated from the compiled data is plotted versus $\varepsilon_{c w, E}$ calculated with Equation 156 for wave and current interaction $\left(U_{c}>0.05\right)$. It seems that the scatter is mainly associated with the Vessem and the Chung et al. (2000) data, which are often overestimated, and with the Bayram et al. (2001) data, which are underestimated. 


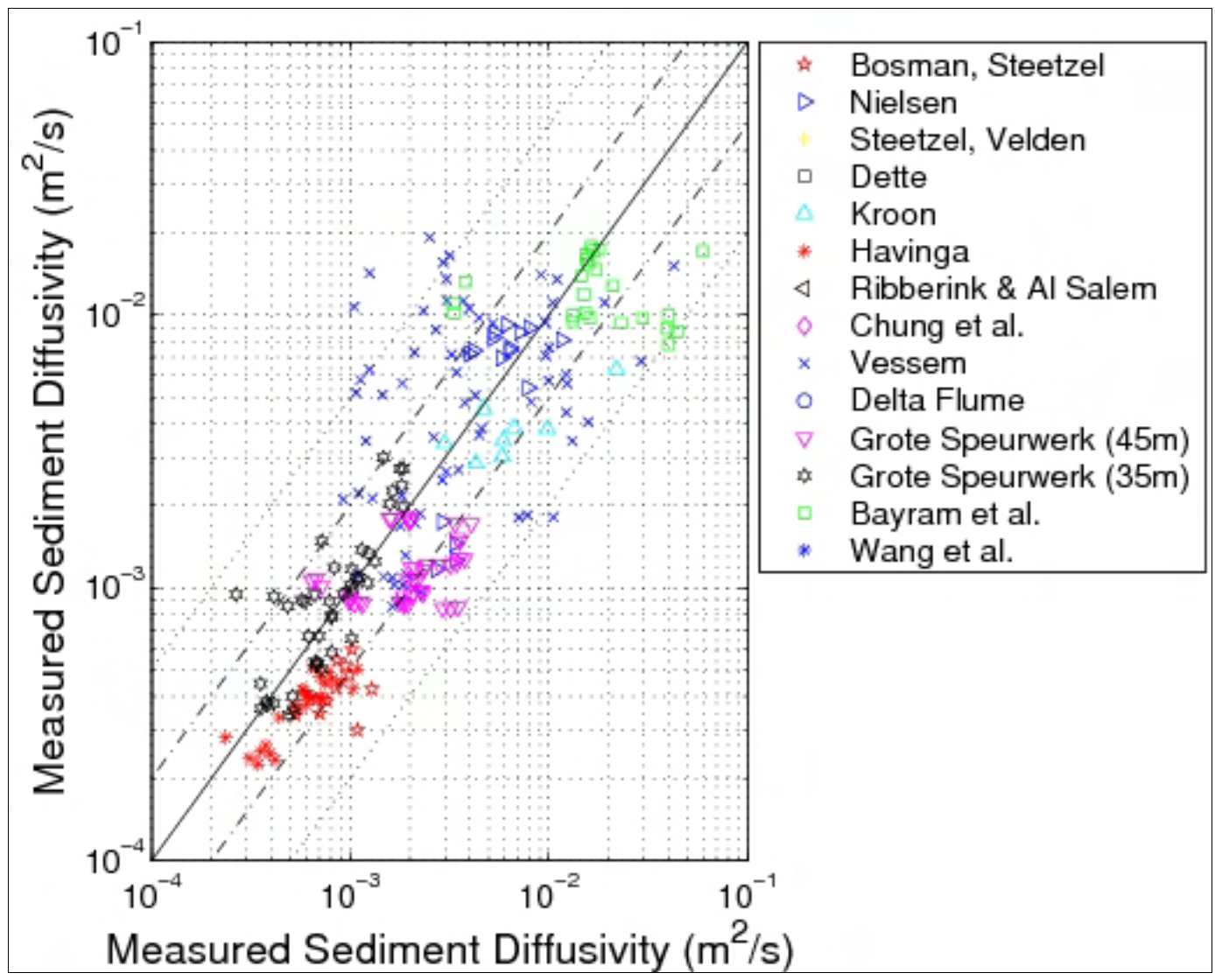

Figure 61 . Vertical sediment diffusivity $\varepsilon_{c w, E}$ estimated from compiled data versus $\varepsilon_{c w, E}$ calculated with Equation 156 for wave and current interaction $\left(U_{c}>0.05\right)$.

\section{Effect of breaking waves on sediment diffusivity}

The vertical distribution of suspended sediment in the surf zone is controlled by the downward settling of sediment particles and the upward mixing of particles due to turbulent diffusion and wave advection. The dominant mechanism for generation of turbulence outside the surf zone is bottom friction, whereas inside the surf zone turbulence is generated both at the seabed by friction and near the water surface by wave breaking. Therefore, large concentrations of sediment can be suspended throughout the water column (Yu et al. 1993; Ogston and Sternberg 2002).

\section{Extension of sediment diffusion expression}

In a study on infilling of navigation channels, Kraus and Larson (2001) employed an exponential concentration profile to estimate the suspended load transport. Wave breaking was assumed to be the main mechanism for the vertical mixing, yielding a concentration profile according to Equation 123 with: 


$$
\varepsilon=\varepsilon_{b}=k_{b}\left(\frac{D_{b}}{\rho}\right)^{1 / 3} h
$$

where $k_{b}$ is an empirical constant, and $D_{b}$ is the energy dissipation due to wave breaking. The expression for $\varepsilon$ produces a constant eddy viscosity over the water depth.

A general expression for $\varepsilon$ should include mixing associated with turbulence from bottom friction and with wave breaking. Also, bottom friction should encompass effects of waves and currents, acting separately or in combination. By exploring the relationship between energy dissipation and shear stress in the bottom boundary layer, the mixing from the bottom turbulence may be expressed through the energy dissipation, which in turn means that this mixing can be combined with the mixing due to breakerinduced turbulence in a straightforward manner. The energy dissipation in the bottom boundary layer under a current may be written:

$$
D_{c}=\tau_{c} u_{*_{c}}
$$

where $u *_{c}$ is the shear velocity due to current only. This expression makes it possible to replace the shear velocity in the eddy viscosity with the energy dissipation.

Equation 158 deviates from the standard way of defining the dissipation due to a current, which should be expressed as the product between a force and a velocity (the mean velocity $U_{c}$ is generally used instead of the shear velocity $u *_{c}$ ). However, using $u *_{c}$ instead of $U_{c}$ yields the same result as the classical mixing length approach:

$$
\begin{gathered}
\varepsilon_{c}=k_{c}\left(\frac{D_{c}}{\rho}\right)^{1 / 3} h=k_{c}\left(\frac{\tau_{c} u_{*_{c}}}{\rho}\right)^{1 / 3} h \\
=k_{c} u_{*_{c}} h
\end{gathered}
$$

Similarly, the energy dissipation in the bottom boundary layer because of the wave motion may be expressed as:

$$
D_{w}=\tau_{w} u_{*}
$$


where $u_{*} w$ is the shear velocity from waves only. The sediment diffusivity due to waves may thus be written:

$$
\begin{aligned}
\varepsilon & =k_{w}\left(\frac{D_{w}}{\rho}\right)^{1 / 3} h \\
& =k_{w} u_{w_{w}} h
\end{aligned}
$$

To employ a general formula for the sediment diffusion, it is natural to assume that:

$$
\varepsilon=\left(\frac{D}{\rho}\right)^{1 / 3} h
$$

where $D$ is the total effective dissipation:

$$
D=k_{b}^{3} D_{b}+k_{c}^{3} D_{c}+k_{w}^{3} D_{w}
$$

in which the energy dissipation from wave breaking $\left(D_{b}\right)$, from bottom friction from a current $\left(D_{c}\right)$, and from waves $\left(D_{w}\right),\left(k_{b}, k_{c}\right.$, and $k_{w}$ are coefficients). The coefficient $k_{b}$ mainly corresponds to an efficiency factor, whereas $k_{c}$ and $k_{w}$ are related to the Schmidt number. Typically, $D_{b}>D_{w}>D_{c}$ and, in many cases, only the largest dissipation needs to be considered. Still, the formula for $\varepsilon$ is simplified because the mixing does not vary through the water column.

Using Equation 134 together with Equation 136 and Equation 154, it is found that:

$$
\begin{aligned}
& k_{c}=\frac{\kappa \sigma_{c}}{6}=\frac{\kappa}{6}\left\{\begin{array}{l}
0.4+3.5 \sin ^{2}\left(\frac{\pi}{2} \frac{W_{s}}{u_{*_{c}}}\right) \text { if } \frac{W_{s}}{u_{*_{c}}} \leq 1 \\
1.0+2.9 \sin ^{2}\left(\frac{\pi}{2} \frac{u_{*_{c}}}{W_{s}}\right) \text { if } \frac{W_{s}}{u_{*_{c}}}>1
\end{array}\right. \\
& k_{w}=\frac{\kappa \sigma_{w}}{3 \pi}=\frac{\kappa}{3 \pi} \begin{cases}0.15+1.5 \sin ^{2}\left(\frac{\pi}{2} \frac{W_{s}}{u_{*_{w}}}\right) & \text { if } \frac{W_{s}}{u_{*_{w}}} \leq 1 \\
1.0+0.65 \sin ^{2}\left(\frac{\pi}{2} \frac{u_{*_{w}}}{W_{s}}\right) & \text { if } \frac{W_{s}}{u_{*_{w}}}>1\end{cases}
\end{aligned}
$$


It is noted that $\left(D_{c} / \rho\right)^{1 / 3}=u *_{c}$ and $\left(D_{w} / \rho\right)^{1 / 3}=u *_{w}$. Furthermore, as shown previously, for the wave and current situation, the same formula as used for $\sigma_{\mathrm{cw}}$ (Equation 156) may be applied to calculate the combined effect of $k_{c}$ and $k_{w}$ (substitute for $\sigma_{c}$ and $\sigma_{w}$, respectively), because the wave-related sediment diffusivity seems to be hindered by the current.

\section{Experimental data with breaking waves}

Table 24 (see also Table 19) summarizes the data sets assembled for investigating breaking effects on the suspended load, where the beach slope, wave properties, and sediment characteristics are listed. These data sets were applicable because they include measurements along cross-shore profiles. Some data sets from three beaches in Great Britain were added by incorporating information from Voulgaris and Collins (2000).

Table 24. Data summary for suspended sediment experiments along beach profiles employed for investigating breaking wave effects (for random waves, $H_{w, \infty}=H_{m o, \infty}$ and $T_{w}=T_{p}$ ).

\begin{tabular}{|l|l|l|l|l|l|l|}
\hline Author(s) & $\begin{array}{l}\text { Number of } \\
\text { Profiles }\end{array}$ & $\begin{array}{l}\text { Beach Slope, } \\
\mathrm{m}(-)\end{array}$ & $d_{50}(\mathrm{~mm})$ & $H_{w, \infty}{ }^{*}(\mathrm{~m})$ & $T_{w}{ }^{*}(\mathrm{sec})$ & $\xi_{\infty}(-)$ \\
\hline Peters (2000) & 9 & $0.02-0.03$ & $0.12-0.33$ & $0.65,1.20$ & 5.5 & $0.12,0.26$ \\
\hline $\begin{array}{l}\text { Voulgaris and Collins } \\
(2000)\end{array}$ & 3 & $\begin{array}{l}0.014,0.020, \\
0.057\end{array}$ & $\begin{array}{l}0.21,0.26, \\
0.33\end{array}$ & $0.30-1.13$ & $3.2-9.1$ & $0.12,0.26$ \\
\hline Bayram et al. (2001) & 6 & 0.03 & $0.18-0.20$ & $1.9-4.5$ & $8.0-12.8$ & $0.12-0.22$ \\
\hline Wang et al (2002) & 2 & $0.03-0.04$ & 0.22 & 0.25 & $1.5,3.0$ & $0.15,0.25$ \\
\hline * For random waves, $H_{w, \infty}=H_{m, \infty}$ and $T_{w}=T_{p .}$. \\
\hline
\end{tabular}

The Irribaren parameter $\xi_{\infty}$ is defined through the offshore wave characteristics and the mean slope of the beach, $m$.

$$
\xi_{\infty}=\frac{m}{\sqrt{H_{w, \infty} / L_{w, \infty}}}
$$

\section{Energy dissipation due to breaking waves}

From a wave transformation model, the estimation of the wave energy dissipation is found from the onshore decrease of the wave energy flux:

$$
D_{b}=-\frac{1}{h} \frac{d F_{w}}{d x}
$$


where $F_{w}=E_{w} C_{g}$ with $E_{w}$ being the wave energy and $C_{g}$ the group celerity. From linear wave theory, $E_{w}$ and $C_{g}$ are obtained as follows:

$$
\begin{gathered}
E_{w}=\frac{1}{8} \rho_{w} g H_{w}^{2} \\
C_{g}=\frac{C}{2}\left[1+\frac{2 k_{w} h}{\sinh \left(2 k_{w} h\right)}\right]
\end{gathered}
$$

where $C=\sqrt{g / k_{w} \tanh (k h)}$ is the wave celerity, with $C \approx \sqrt{g h}$ in shallow water. The $x$-axis points onshore.

If the cross-shore variation in wave height is not measured (or measured at a spatial scale not fine enough to calculate the energy dissipation), it is still possible to estimate the dissipation using theoretical models. One classical approach is to adopt the analogy with the energy dissipation of a bore or a hydraulic jump (Svendsen 1984):

$$
D_{b}=A_{\varepsilon} \rho_{w} g h \frac{H_{w}^{3}}{T_{w}\left(4 h^{2}-H_{w}^{2}\right)}
$$

where $A_{\varepsilon}$ is a coefficient that accounts for the difference between the dissipation in a bore and that in a classical hydraulic jump. Stive (1984) proposed the following relationship:

$$
A_{\varepsilon}=2 \tanh \left(5 \xi_{\infty}\right)
$$

Equation 172 is based on a monochromatic breaking wave. For random waves, a coefficient should be added to take into account the percentage of breaking waves (Larson 1995):

$$
\alpha_{b}=\exp \left[-\left(\frac{\gamma_{b} h}{H_{r m s}}\right)^{2}\right]
$$

where $\gamma_{b}$ is the breaker depth ratio, and $H_{r m s}$ is the significant root-meansquare wave height neglecting breaking. 
Figure 62 plots a comparison between the estimated energy dissipation from the measured wave height variation and the calculated energy dissipation from the bore analogy using the data from Peters (2000), who carried out an experiment in a large wave flume over a sandy bed. The characteristics of the experiment are listed in Tables 19 and 24. A clear correlation is found between these two methods for estimating the wave energy dissipation. The scatter is mainly caused by the limited number of data points employed in the first method and the limitations of the theory for the bore analogy. Furthermore, the wave energy dissipation derived from wave measurements includes both bottom friction and wave breaking. The former might be small, but probably contributes to the scatter.

\section{Influence of Irribaren parameter and $u_{*_{w}} / W_{s}$ on sediment diffusivity}

Equations 157, 172, and 174 were used to estimate the sediment diffusivity due to wave breaking. As a first approximation, the efficiency parameter was assumed to be a constant and estimated from the data:

$$
k_{b}=0.010
$$

Assuming that wave breaking is the main mechanism for the sediment diffusion (i.e., prevails over the shear diffusivity due to current and waves), Equation 157 should correctly predict the sediment diffusivity estimated from the concentration profiles of the experiments. Figure 63 plots (a) the ratio $\varepsilon_{b \text {,meas }} / \varepsilon_{b \text {,pred }}$ versus the Irribaren parameter $\xi_{\infty}$, and $(b)$ the ratio $u^{*}{ }^{*} / W_{s}$, where $\varepsilon$, meas is the estimated sediment diffusivity from the measured concentration profiles, and $\varepsilon_{b, p r e d}$ is the calculated sediment diffusivity using the energy dissipation from breaking. Satisfactory agreement is observed even if these results indicate either an increasing function of the Irribaren parameter or a decreasing function of the ratio $u^{*} w_{w} / W_{s}$. 


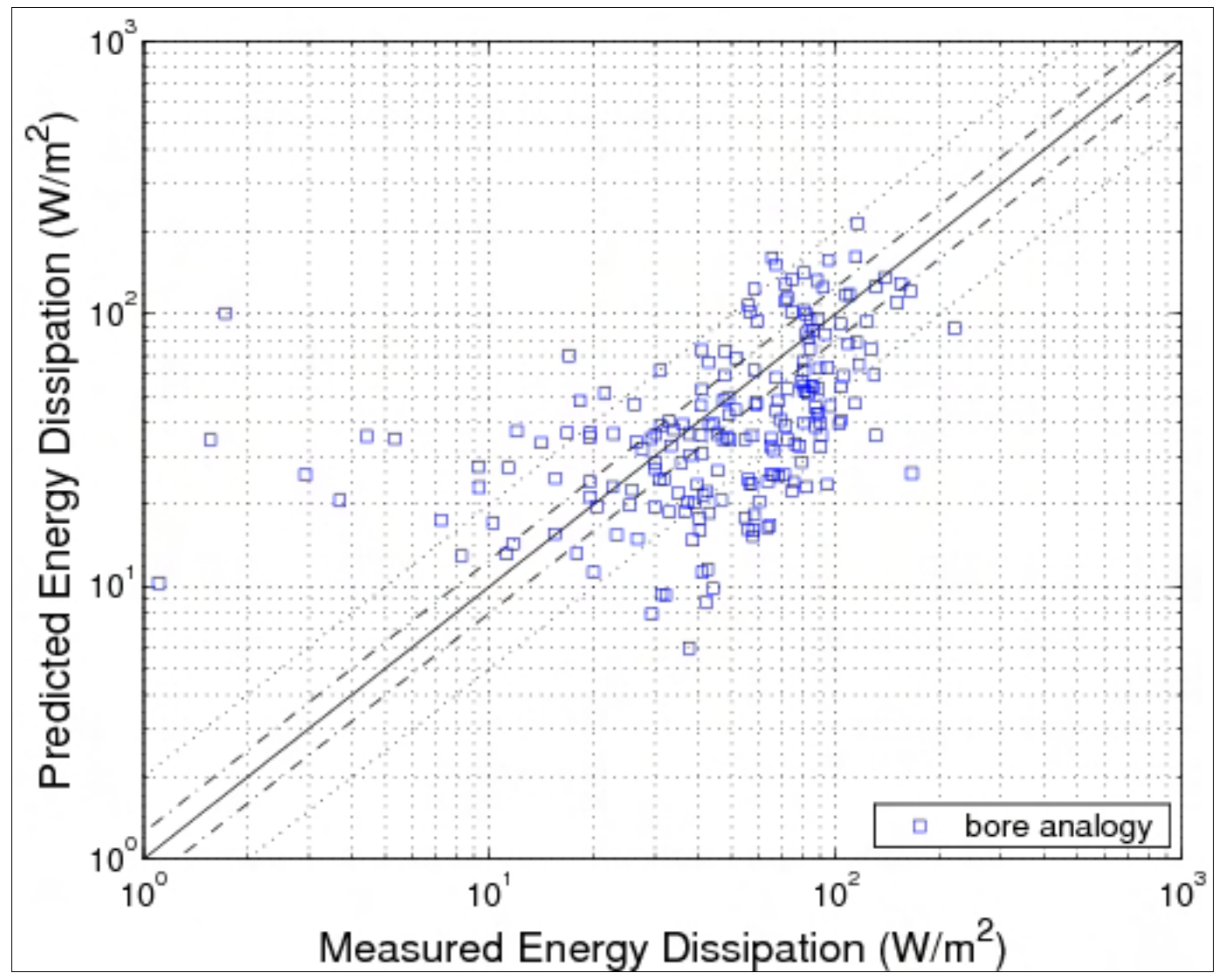

Figure 62. Comparison between estimated energy dissipation from measured wave height variation and calculated energy dissipation from bore analogy using data from Peters (2000).

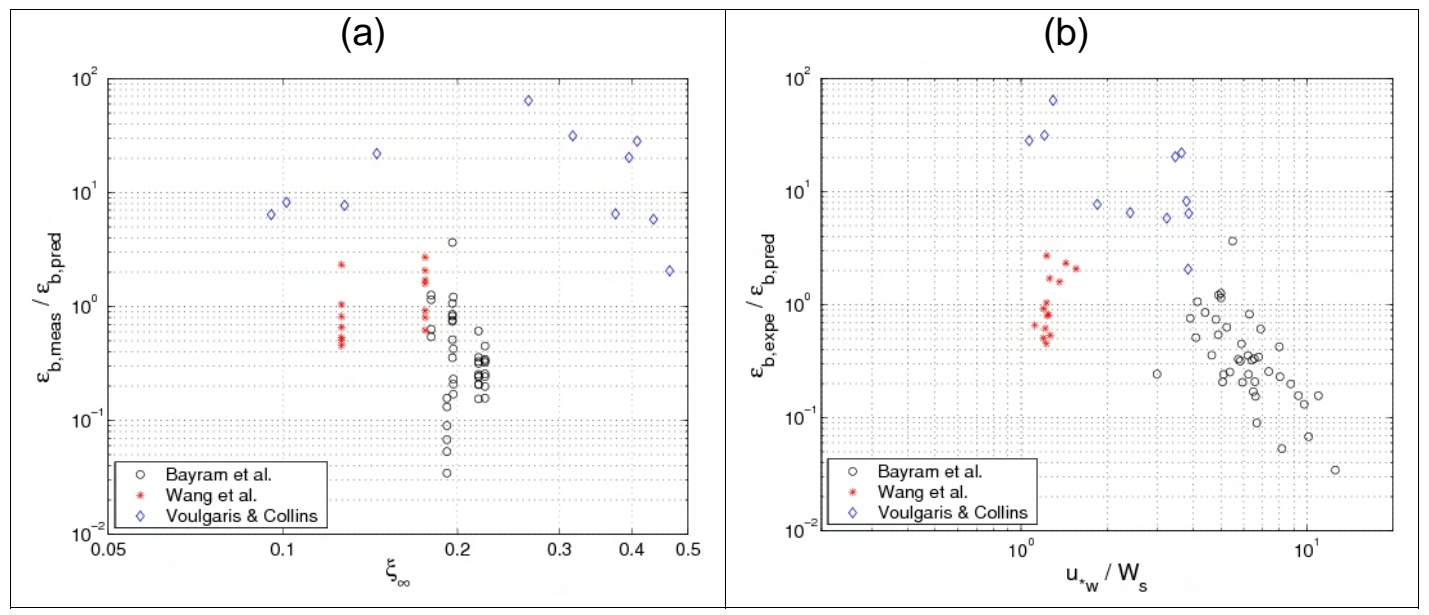

Figure 63. (a) Ratio $\varepsilon_{b, \text { meas }} / \varepsilon_{b, p r e d}$ versus Irribaren parameter $\xi \infty$, and (b) ratio $u *_{w} / W_{s}$ (b) using data from Table 24 (except Peters data). 
The Peters (2000) data seem to be insensitive to two main parameters (Shields parameter, grain size). Indeed, the slope a of the concentration profile (assuming an exponential profile) only varies from 1 to 8 for the entire data set, whereas $\theta_{c w, m}$ varies from 0.3 to 2 ( $U_{w}$ varies from 0.5 to 1.25) and $d *$ varies from 3 to 8 . As the measured concentration profiles were not provided, the results from this data set were not considered in further analysis.

It seems that the influence of the ratio $u^{*} w / W_{s}$ is stronger than the influence of the Irribaren parameter. An empirical coefficient was determined following the procedure for a current or waves only. Assuming that $\sigma_{B}=1$ is $u^{*}{ }_{w} / W_{s}=0$, a new equation for $k_{b}$ when proposed:

$$
k_{b}=0.062\left[1-0.9 \tanh \left(0.25 \frac{u_{*_{w}}}{W_{s}}\right)\right]
$$

Employing the Irribaren parameter instead, the following equation may be used:

$$
k_{b}=0.012\left[1+1.0 \tanh \left(\xi_{\infty}\right)\right]
$$

In Table 25, Equations 175, 176, and 177 yield good agreement with the data for the prediction of sediment diffusivity in case of breaking waves. However, Equations 176 and 177 do not improve the results compared to Equation 175, as expected. Thus, $k_{b}$ appears to be independent of the Irribaren parameter and the type of breaker, which seems to be unrealistic. This coefficient not being dependent on breaker type may be explained by the fact that turbulence due to the breakers affects most of the water column and the percentage of the available energy dissipation for sediment diffusion compared to the total energy dissipation $\left(D_{b}\right)$ does not depend on breaker type. However, the type of wave breaking should be characterized by the wave transformation model employed, for example, through the breaker depth ratio and the energy dissipation coefficient. Thus, it is implicitly included in the sediment diffusivity. 
Table 25. Prediction of sediment diffusivity for transport under breaking waves.

\begin{tabular}{|l|l|l|l|l|}
\hline Equation & $P \times 2(\%)$ & $P \times 5(\%)$ & Mean $\left(f\left(c_{R}\right)\right)$ & $\operatorname{Std}\left(f\left(c_{R}\right)\right)$ \\
\hline$k_{b}=0.010$ & 73 & 96 & 0.02 & 0.31 \\
\hline$k_{b}$ from Equation 176 & 76 & 96 & -0.01 & 0.29 \\
\hline$k_{b}$ from Equation 177 & 74 & 96 & -0.02 & 0.30 \\
\hline
\end{tabular}

Figure 64 plots the vertical sediment diffusivity $\varepsilon$ estimated from the compiled data plotted versus $\varepsilon$ calculated with Equation 157 and $k_{b}=0.010$ for the cases where breaking waves occurred. The results depend on the data set: the data from Vessem and Chung et al. (2000) are in general overestimated by Equation 157, whereas the data from Bayram et al. (2001) are typically underestimated. However, compared with all data, Equation 157 yields satisfactory results (see Table 25).

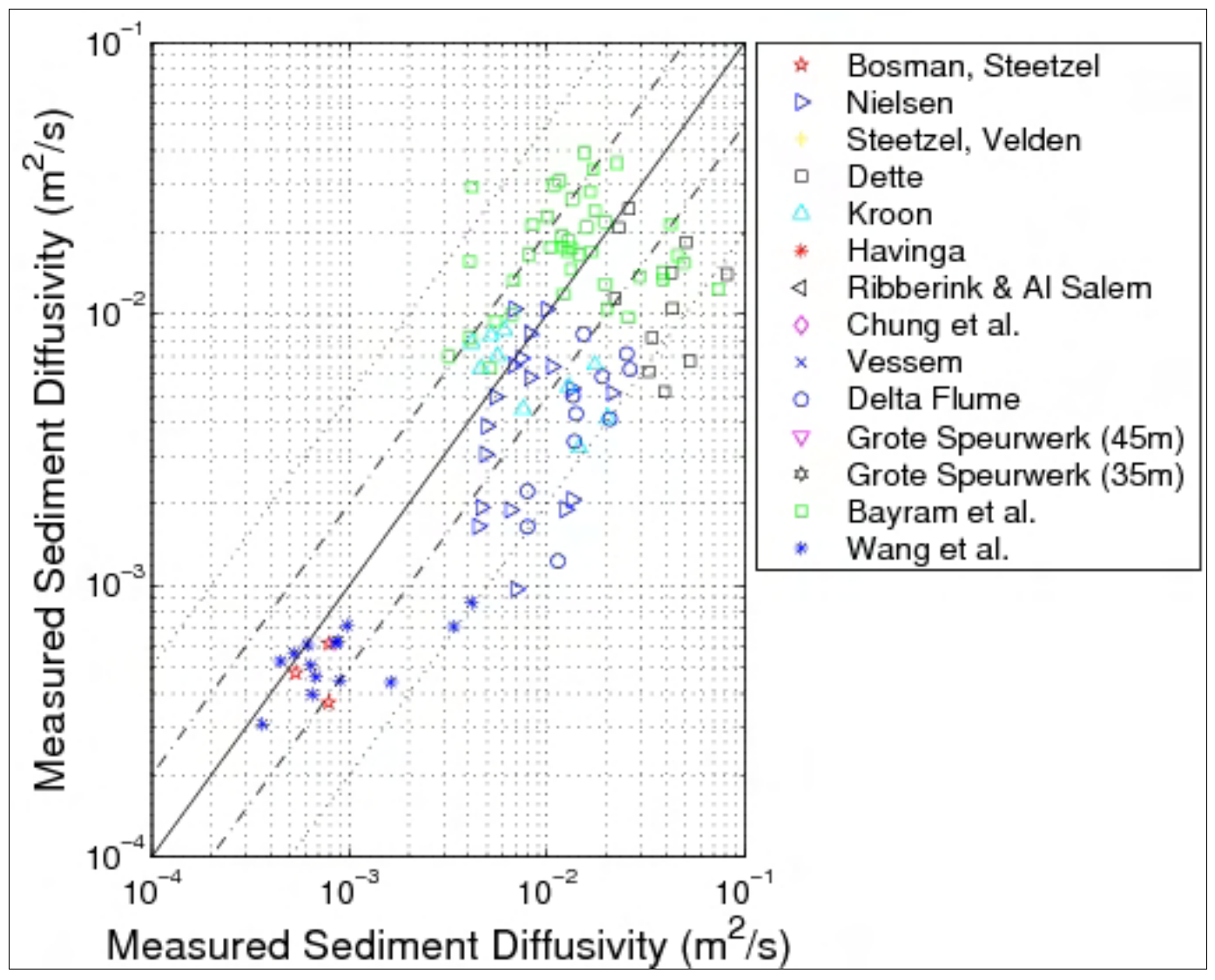

Figure 64 . Vertical sediment diffusivity $\varepsilon_{V, E}$ estimated from compiled data versus $\varepsilon_{V, E}$ calculated with Equations 157 and 175 for breaking waves. 


\section{Reference concentration}

The reference concentration strongly depends on the hypothesis employed for the concentration profile. If a power-law profile is assumed, the reference concentration not only depends on the hydrodynamics and sediment parameters, but also on the reference level $z_{a}$ that has been chosen. The most logical assumption for the reference level $z_{a}$ is the upper-edge of the bed-load layer $\delta_{b}$ (Van Rijn 1993). For an exponential profile, there is no need to choose a reference level, as the concentration is a known value for $z=0$. For flat beds, for a saltation regime, Van Rijn (1984a, b) proposed,

$$
\delta_{b}=0.3 d_{50} d_{*}^{0.7} T_{\tau}^{0.5}
$$

where $T_{\tau}=\left(\theta-\theta_{c r}\right) / \theta_{c r}$ is the dimensionless bed-shear stress parameter. This equation yields a value for $\delta_{b}$ close to the Nikuradse roughness $k_{s}$, i.e., $\delta_{b}=2$ to $15 d_{50}$. In the presence of bed forms, Van Rijn $(1984 b, 1993)$ proposed to use for reference level the maximum value between $k_{s}$ and half of the size of the bed forms $1 / 2 H_{r}$. Furthermore, for sheet-flow transport, Wilson (1989a) showed that the bed-load layer thickness is proportional to the Shields parameter $\theta$. All these parameters should induce large uncertainty in the prediction of the reference concentration depending on the sediment transport regimes and assumption made by the authors.

Figure 65 plots the observed reference concentration $c_{R}$ assuming an exponential profile compared to the reference concentrations $c_{a}$ assuming a power-law profile (linear and parabolic) at the reference level $z_{a}=k_{s}$ $=2 d_{50}$. The data plotted in this figure correspond to a steady current. It shows the large differences observed for the reference concentration depending on the choice of the concentration profile. Thus, a parabolic concentration profile produces a reference concentration from a factor 2 to a factor 500 greater than the reference concentration from the exponential profile. 


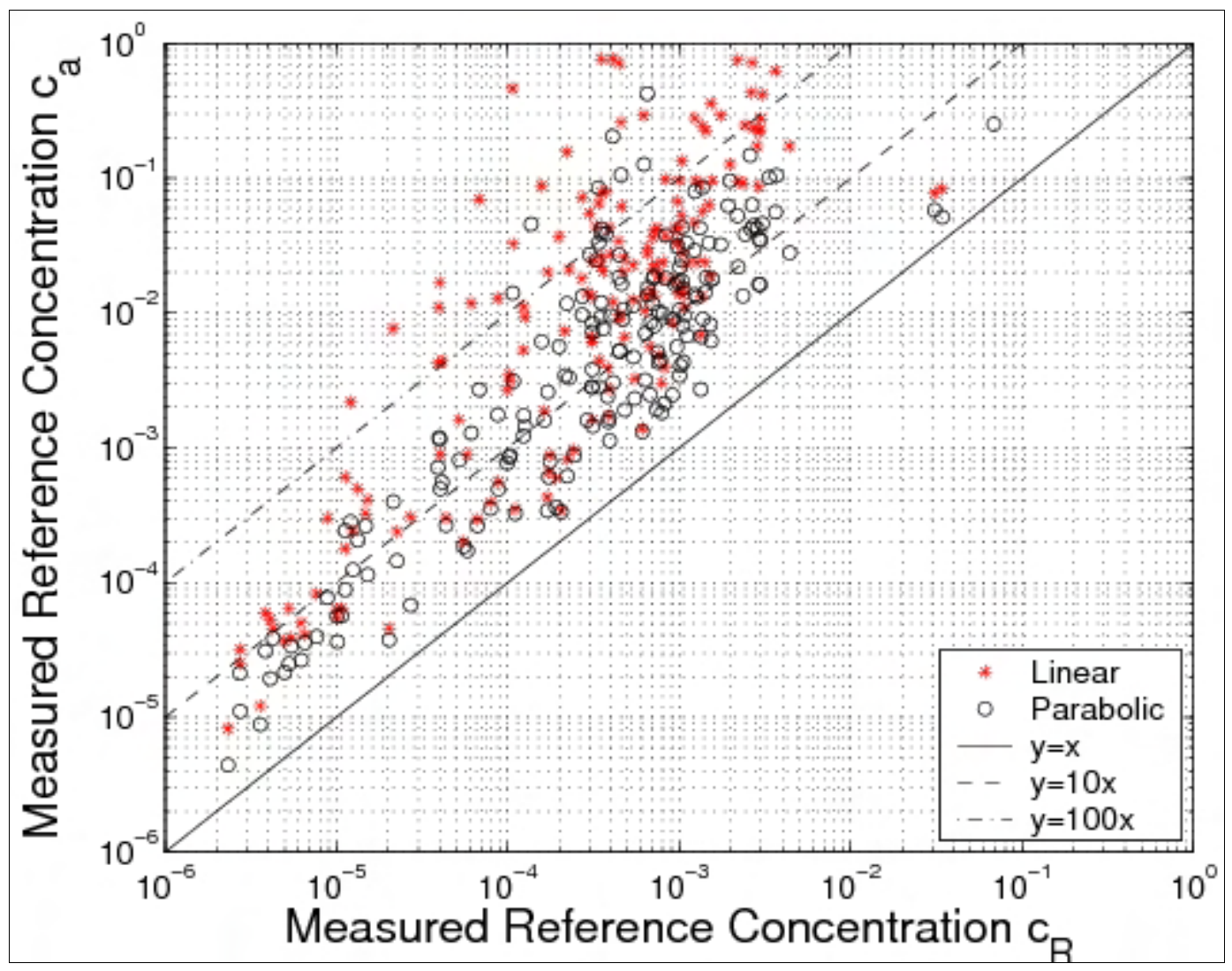

Figure 65. Comparison between observed reference concentrations assuming an exponential profile $\left(c_{R}\right)$ or power-law profile $\left(c_{a}\right)$ at reference level $z_{a}=k_{s}=2 d_{50}$.

\section{Effect of current}

Several formulas have been proposed to estimate the reference concentration at the bottom. The maximum volume concentration is commonly assumed to be $c_{m}=0.65$. Madsen (1993) proposed a reference concentration based on an exponential concentration profile, whereas previous relationships were mostly based on power-law profiles or on the mean bed-load concentration. Table 26 presents some of the most common formulas for $c_{a}$ and $c_{R}$. Most of the formulas (except the second one from Van Rijn (1984b)) assume a plane bed and use the skin Shields parameter $\theta^{\prime}$ (calculated with $k_{s}=2 d_{50}$ ). 
Table 26. Selected relationships for reference concentration (chronological order).

\begin{tabular}{|c|c|c|}
\hline Author(s) & Equation & Equation Number \\
\hline Engelund and Fredsøe (1976) & $\begin{array}{l}c_{a}=0.65\left(1+\lambda^{-1}\right)^{-3} \\
\text { with } \lambda=4.3\left(\frac{\theta_{c}^{\prime}-\theta_{c r}-0.26 p}{\theta_{c}^{\prime}}\right)^{0.5} \\
\text { and } p=\left[1+\left(\frac{0.26}{\theta_{c}^{\prime}-\theta_{c r}}\right)^{4}\right]-0.25\end{array}$ & $(179)$ \\
\hline Smith and McLean (1977) & $\begin{array}{l}c_{a}=0.004 c_{m}\left(\frac{T_{\tau}}{1+0.004 T_{\tau}}\right) \\
\text { with } T_{\tau}=\frac{\theta_{c}-\theta_{c r}}{\theta_{c r}}\end{array}$ & $(180)$ \\
\hline Van Rijn (1984a) & $c_{a}=0.18 c_{m} \frac{T_{\tau}}{d_{*}}$ & $(181)$ \\
\hline Van Rijn (1984b) & $\begin{array}{l}c_{a}=0.015 c_{m} \frac{d_{50}}{z_{a}} \frac{T_{\tau}^{1.5}}{d_{*}^{0.3}} \\
\text { with } z_{a}=\max \left(k_{s}, 1 / 2 H_{r}\right)\end{array}$ & $(182)$ \\
\hline Zyserman and Fredsøe (1994) & $c_{a}=\frac{0.331\left(\theta_{c}^{\prime}-\theta_{c r}\right)^{1.75}}{1+0.72\left(\theta_{c}^{\prime}-\theta_{c r}\right) 1.75}$ & $(183)$ \\
\hline Madsen (1993) & $\begin{array}{l}c_{R}=\frac{2}{\pi} \gamma_{0} c_{o} T_{\tau} \\
\left.\text { with } \gamma_{0}=2 \cdot 10^{-4} \text { (plane bed }\right)\end{array}$ & $(184)$ \\
\hline
\end{tabular}

Figure 66 plots the different formulas investigated versus the total Shields parameter. It appears that large variations occur depending on the formula used. If the methods of Engelund and Fredsøe (1976) and Van Rijn (1993) yield results which are in reasonably good agreement, there is still a difference which can reach a factor 5 . It should also be noted that the two Van Rijn formulas reach the maximum value $c_{m}$ if $\theta_{c} \approx 1$, i.e., when the sheet-flow regime occurs. The methods of Zyserman and Fredsøe (1994) and Smith and McLean (1977) yield smaller bed concentration. Van Rijn (1993) explained that this is because Smith and McLean (1977) defined $c_{a}$ at a higher level. Based on these results, it appears that the calculation of the reference concentration induces large uncertainty. 


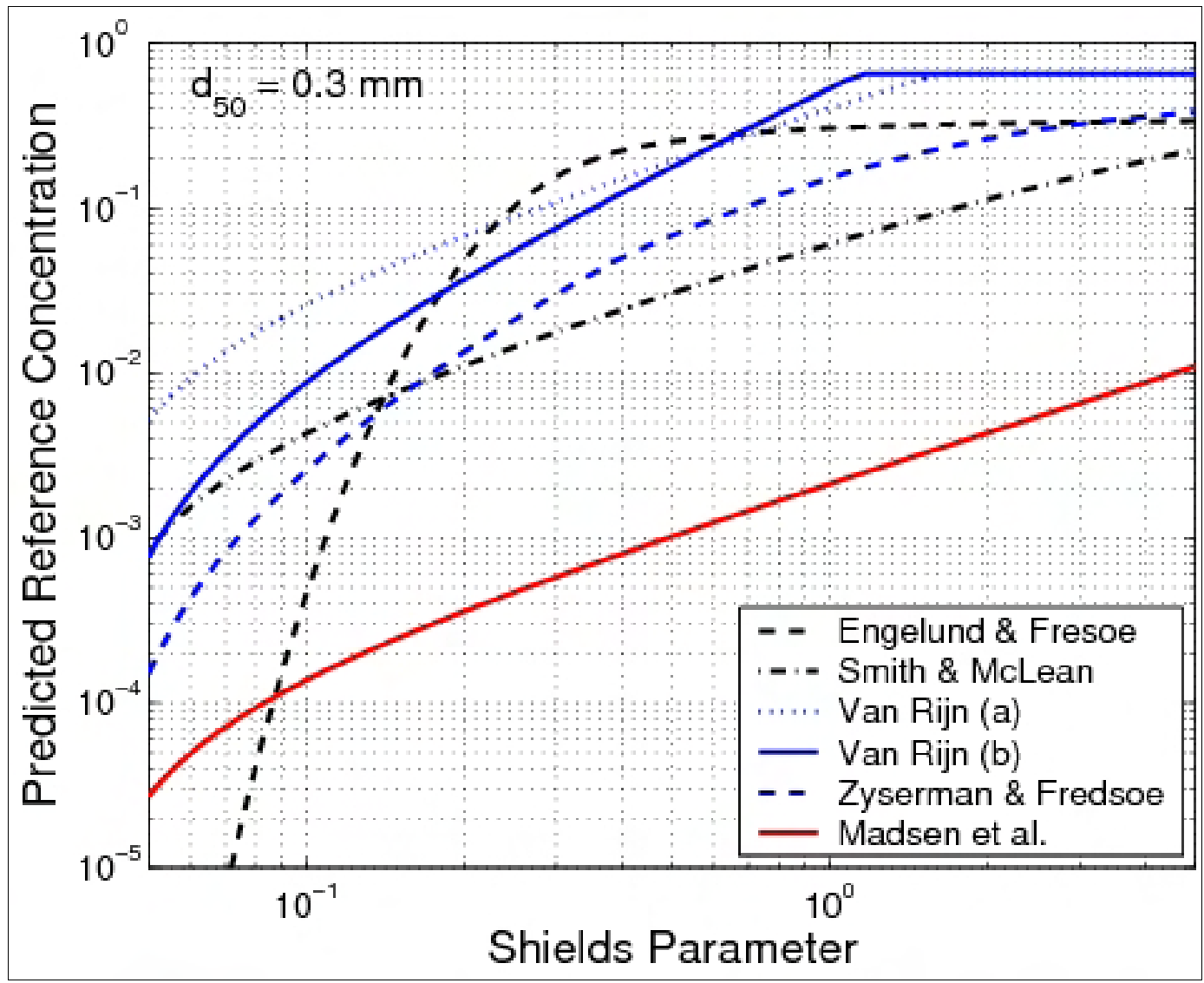

Figure 66. Predicted reference concentration $c_{a}$ and $c_{R}$ versus Shields parameter from various formulas.

Formulas using power-law methods are not attractive for the reason that the accuracy depends on the choice of the reference level. Finally, using the expression of Madsen (1993) based on an exponential sediment concentration profile, a difference of one to two orders of magnitude is observed, as expected from Figure 65.

For all the formulas, a comparison was made with the measurements for the bottom concentration using data from Table 15. These values were calculated assuming a parabolic power-law profile with $z_{a}=k_{s}$ (for comparison with Equations 179, 180, 181, 182, and 183) or an exponential profile (for comparison with Equation 184). The percentage of values obtained with an error less than factor 2 or 5 (designed as $P x 2$ or $P x 5$ ) as well as the mean value and the standard deviation of the function $f\left(c_{R}\right)=\log \left(c_{a / R, p r e d} / c_{a / R, \text { meas }}\right)$ are listed in Table 27. Predictions using Equations 179, 180, 181, 182, and 183 are overestimated, especially for small values of $c_{a}$. The formula of Smith and McLean (1977) gives the best results among the compared formulas. It appears that the Madsen (1993) 
Table 27. Prediction of reference concentration assuming parabolic power-law or an exponential sediment concentration profile.

\begin{tabular}{|c|c|c|c|c|}
\hline Author(s) & $P \times 2$ & $P \times 5$ & $\operatorname{Mean}\left(f\left(c_{a / R}\right)\right)$ & $\operatorname{Std}\left(f\left(C_{a / R}\right)\right)$ \\
\hline Engelund and Fredsøe ${ }^{1}$ & 10 & 22 & 0.56 & 1.18 \\
\hline Smith and McLean ${ }^{1}$ & 21 & 51 & 0.60 & 0.75 \\
\hline Van Rijn $(a)^{1}$ & 01 & 05 & 1.62 & 0.67 \\
\hline $\operatorname{Van}$ Rijn (b) ${ }^{1}$ & 05 & 17 & 1.19 & 0.85 \\
\hline Zyserman and Fredsøe $^{1}$ & 11 & 34 & 0.81 & 0.79 \\
\hline Madsen $^{2}$ & 27 & 50 & 0.75 & 0.83 \\
\hline Equations 185 and $186^{2}$ & 49 & 84 & -0.07 & 0.51 \\
\hline Equation 185 with $A_{c r}=510^{-42}$ & 28 & 65 & 0.05 & 0.77 \\
\hline
\end{tabular}

formula yields correct results compared to the measurements for $c_{R}$ assuming an exponential profile. However, this formula does not seem to be sufficiently sensitive to the Shields parameter: it gives more or less a constant value for each data set.

Following Madsen et al. (2003), the reference volumetric bed concentration may be estimated from the volumetric bed load, assuming $q_{s}=c_{R}$ $U_{s}$. The bed load may be written based on the results by Camenen and Larson (2005a, b), namely $q_{s} \propto \theta^{3 / 2} \exp \left(-4.5 \theta_{c r} / \theta\right)$. Madsen (1993) proposed, as a first approximation, that the speed of the bed-load layer may be proportional to the shear velocity, as $U_{s} \propto \theta^{1 / 2}$. The bed reference concentration may thus be written as follows:

$$
c_{R}=A_{c R} \theta_{T} \exp \left(-4.5 \frac{\theta_{c r}}{\theta_{M}}\right)
$$

where $\theta_{T}$ is the transport-dependent Shields parameter, and $\theta_{M}$ is the maximum Shields parameter to compare with the critical Shields parameter for the inception of the sediment motion (Soulsby 1997; Camenen and Larson 2005a, b). For a current only, $\theta_{M}=\theta_{T}=\theta_{c}$ where $\theta_{c}$ is the current-related Shields parameter.

Using measurements for transport under a steady current, the coefficient $A_{c R}$ is found to vary from $510^{-6}$ to $41^{-2}$. Employing a constant value $A_{c R}=510^{-4}$ produces better results than the Madsen (1993) formula 
(Table 27). Some authors have found that the reference concentration may be more sensitive to the bed shear stress (see Equation 182 by Van Rijn (1984b), and Equation 183 by Zyserman and Fredsøe (1994)). Van Rijn $(1984 a, b)$ observed that the reference concentration $c_{a}$ is a function of the dimensionless grain size $d *$, but to a varying power depending on the presence or not of bed forms. For the compiled data (see Table 15), the dimensionless grain size $d *$ varies from 1 to 18 . Improved results were obtained by calibrating $A_{c R}$ for current as a function of the dimensionless grain size:

$$
A_{c R, c}=3.5 \cdot 10^{-3} \exp \left(-0.3 d_{*}\right)
$$

Figure 67 plots the predicted reference concentration $c_{R}$ using Equations 185 and 186 versus the estimated reference concentration assuming an exponential profile. Even if the results are overall in agreement, it seems as the sensitivity to the Shields parameter should be greater. The reference concentration is typically overestimated for weak shear stress. The prediction of the reference concentration is significantly improved compared to the Madsen (1993) formula: nearly 50 percent of the data are predicted within a factor 2 and 85 percent within a factor 5 . The mean value of $f\left(c_{R}\right)$ is closer to zero, and its standard deviation is reduced compared to the other formulas compared. However, some dispersion appears for the data sets of Voogt et al. (1991) and Damgaard et al. (2003), which produces a negative value on the mean of $f\left(c_{R}\right)$.

\section{Effect of waves}

For waves only over flat beds and rippled beds, Nielsen (1986, 1992, pp. 201-233) observed that an exponential profile describes the concentration profile well. He found that the bottom concentration $\left(c_{0}\right)$ is a function of the Shields parameter:

$$
\begin{gathered}
c_{0}=0.007\left(\theta_{w}^{\prime}-\theta_{c r}\right)^{1.5}, \text { for flat beds } \\
c_{0}=0.05 \theta_{r}^{3}, \quad \text { for rippled beds }
\end{gathered}
$$

where $\theta_{r}$ is the enhanced skin Shields parameter due to the ripples based on the study by DuToit and Sleath (1981): 


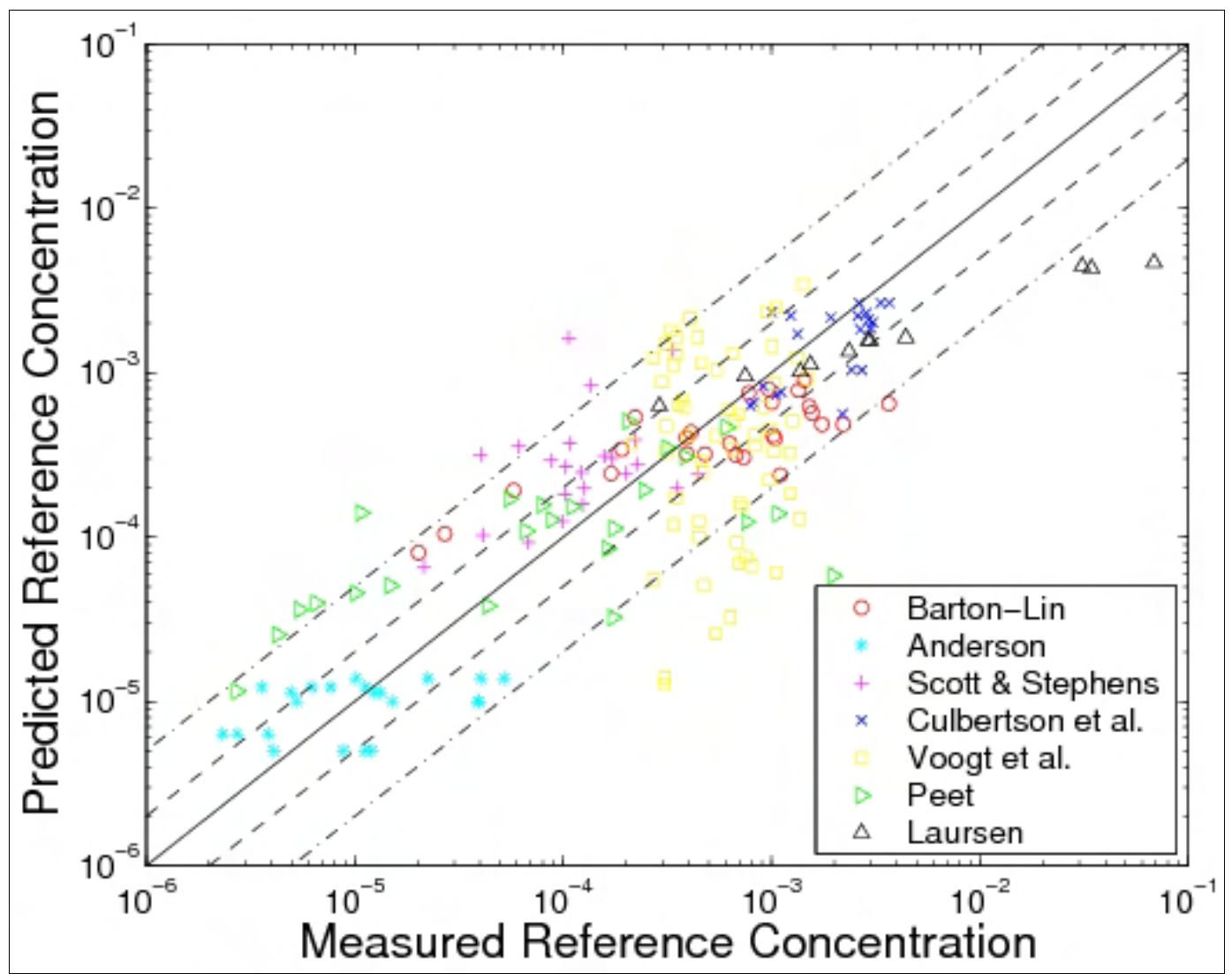

Figure 67. Predicted reference concentration $c_{R}$ using Equations 185 and 186 versus experimental reference concentration assuming an exponential profile for concentration.

$$
\theta_{r}=\frac{\theta_{w}^{\prime}}{\left(1-\pi H_{r} / L_{r}\right)^{2}}
$$

Nielsen (1986) collected experimental data to fit Equations 187 and 188. These data sets are summarized in Table 28.

Table 28. Experimental data used by Nielsen (1986).

\begin{tabular}{|l|l|l|l|}
\hline Author(s) & $d_{50}(\mathrm{~mm})$ & $T_{\mathrm{w}}(\mathrm{sec})$ & Regime \\
\hline Homma et al. (1965) & 0.20 & $1.0-1.8$ & Ripples \\
\hline Nakato et al. (1977) & 0.14 & $1.2-2.4$ & Ripples \\
\hline Nielsen (1979) & $0.08-0.55$ & $1.3-3.0$ & Ripples \\
\hline Sleath (1982) & 0.41 & $3.0-3.6$ & Ripples \\
\hline Horikawa et al. (1982) & 0.20 & $2.0-6.0$ & Sheet flow \\
\hline Hayakawa et al. (1983) & 0.27 & $4.0-6.0$ & Ripples \\
\hline Staub et al. (1984) & 0.19 .0 .36 & $6.8,9.1$ & Sheet flow \\
\hline
\end{tabular}


The observed bottom sediment concentration due to waves is much greater compared to the concentration due to a current and it seems to be more sensitive to the total shear stress (Equations 187 and 188 proposed by Nielsen (1986)). Figure 68 presents the relationship between the bottom concentration $c_{o}$ (which should be similar to the reference concentration $c_{R}$ because Nielsen (1986) estimated $c_{o}$ from an exponential concentration profile) and the modified skin Shields parameter $\theta_{r}$ (which should be similar to the total Shields parameter) for both rippled beds and sheet flow.

Using the same data set as Nielsen (1986), a similar equation to Equation 185 could be developed. Following the results by Camenen and Larson (2005b) on bed load transport, the mean shear stress is used for the transport-dependent term $\theta_{T}=\theta_{w, m}$, whereas the maximum wave shear stress is used for the effect of the critical shear stress $\left(\theta_{M}=\theta_{w}\right)$ :

$$
c_{R}=A_{c R, w} \theta_{w, m} \exp \left(-4.5 \frac{\theta_{c r}}{\theta_{w}}\right)
$$

where $\theta_{w, m}=\frac{1}{T_{w}} \int_{0}^{T_{w}} 1 / 2 f_{w} u_{w}^{2}(t) /\left[(s-1) g d_{50}\right] d t\left(\theta_{w, m}=1 / 2 \theta_{w}\right)$ for sinusoidal wave velocity.

However, it seems that the effect of the dimensionless grain size is not as strong as for steady current data and the reference concentrations obtained by Nielsen (1986), are often greater compared to the steady current data set $\left(210^{-4}<A_{c R}<410^{-2}\right)$. Indeed, using Equation 190 with Equation 186 produces large underestimations. One explanation could be that the mean shear stress enters instead of the maximum shear stress, although it cannot explain the entire underestimation. Thus, the following calibration is proposed based on Nielsen (1986) results:

$$
A_{c R, w, \text { Nielsen }}=2.010^{-2} \exp \left(-0.2 d_{*}\right)
$$




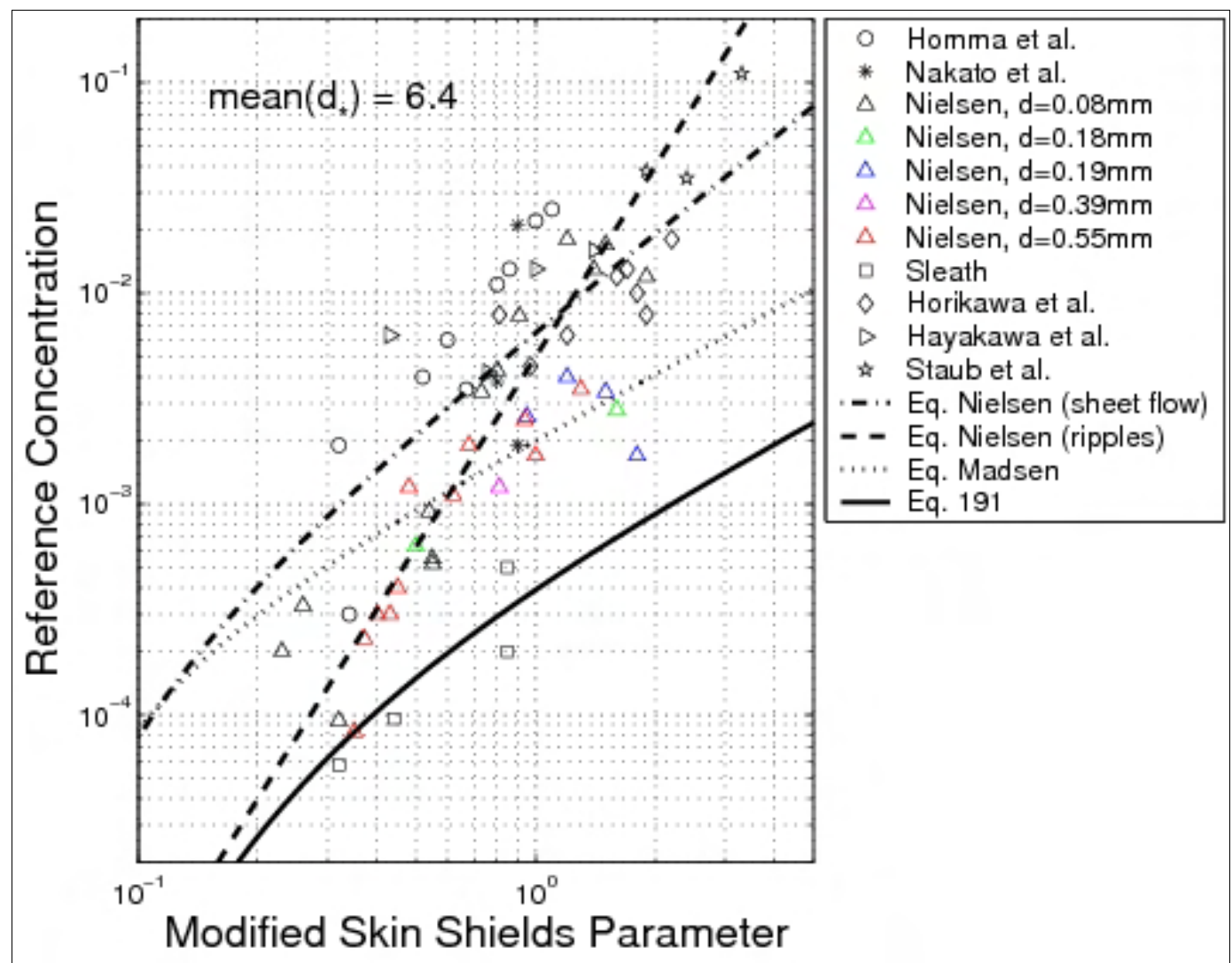

Figure 68. Bottom concentration co versus modified skin Shields parameter $\theta_{r}$ using data collected by Nielsen (1986); new equation is based on Equation 190 with calibrated coefficient value from Equation 191 (curves are plotted using a mean value for $d_{\star}$ ).

It should be noted that $\theta_{c r} \approx 0.055$ for all the data (fine sediments), but $d *$ varies from 2 to 14 (Figure 68). The mean value $d *=6.4$ was used for plotting Equation 190.

The percentage of values predicted within a factor 2 or 5 as well as the mean value and the standard deviation of the ratio $f\left(c_{R}\right)=$ $\log \left(c_{R, p r e d} / c_{R, \text { meas }}\right)$ are presented in Table 29. Again, the Madsen (1993) formula (Equation 184) yields a good comparison to the measured values for $c_{R}$. However, as before, this formula seems not to be sufficiently sensitive to the Shields parameter and grain size giving more or less constant value for each data set. Equation 190 together with Equation 191 present equivalent results as Equation 188. Using a power $n=1.5$ instead of $n=1.0$ on the transport-depending term in Equation 190 may improve the behavior of the relationship in comparing with the Nielsen (1986) data. 
Table 29. Prediction of bottom concentration using data from Nielsen (1986).

\begin{tabular}{|l|l|l|l|l|}
\hline Author(s) & $P \times 2(\%)$ & $P \times 5(\%)$ & Mean $\left(f_{\left.C_{R}\right)}\right)$ & $\operatorname{Std}\left(f\left(c_{R}\right)\right)$ \\
\hline Nielsen & 47 & 85 & 0.02 & 0.50 \\
\hline Madsen & 26 & 63 & -0.18 & 0.64 \\
\hline Equations 190 and 186 & 11 & 29 & -0.98 & 0.50 \\
\hline Equations 190 and 191 & 50 & 89 & 0.06 & 0.45 \\
\hline
\end{tabular}

Finally, using previously presented data sets where waves were dominant $\left(U_{c}<0.05 \mathrm{~m} / \mathrm{sec}\right.$ ), a comparison was performed between the different formulas investigated (Table 30). A greater dispersion is observed compared to the Nielsen (1986) data set, mainly because many data points originate from field experiments where larger uncertainty typically occurs. This may explain the poorer results obtained with Equations 190 and 191.

Table 30. Prediction of reference concentration using studied data sets encompassing waves only.

\begin{tabular}{|c|c|c|c|c|}
\hline Author(s) & $P \times 2(\%)$ & $P \times 5$ (\%) & Mean $\left(f\left(c_{R}\right)\right)$ & $\operatorname{Std}\left(f\left(c_{R}\right)\right)$ \\
\hline Nielsen $^{1}$ & 24 & 55 & 0.46 & 1.05 \\
\hline Madsen $^{1}$ & 21 & 49 & 0.70 & 0.58 \\
\hline Equations 190 and $186^{1}$ & 41 & 77 & -0.15 & 0.59 \\
\hline Equations 190 and $191^{1}$ & 15 & 38 & 0.80 & 0.58 \\
\hline Equation 190 with $A_{c r}=510^{-41}$ & 32 & 68 & -0.38 & 0.58 \\
\hline Equations 190 and $C \& L^{1}$ & 37 & 72 & -0.31 & 0.59 \\
\hline Equations 190 and $186^{2}$ & 35 & 70 & 0.06 & 0.63 \\
\hline Equations 190 and $191^{2}$ & 11 & 29 & 1.03 & 0.61 \\
\hline Equation 190 with $A_{c r}=510^{-4} 2$ & 36 & 71 & -0.17 & 0.61 \\
\hline Equation 190 and $C \& L^{2}$ & 38 & 71 & -0.10 & 0.61 \\
\hline \multicolumn{5}{|c|}{$\begin{array}{l}{ }^{1} \text { Van Rijn (1993) method used for both ripple characteristics and Nikuradse roughness. } \\
2 \text { Soulsby and Whitehouse (2005a, b) equations used for ripple characteristics and Kim } \\
\text { (2004) equation for the Nikuradse roughness. } \\
\text { C\&L: } A_{C R} \text { given by Camenen and Larson (2007). }\end{array}$} \\
\hline
\end{tabular}

Even if the range of grain sizes is quite similar to the Nielsen (1986) data set ( $d *$ varies from 1.6 to 11), it seems that Equation 190 together with Equation 191 overestimate the measurements. An explanation for this overestimation using Equation 191 may lie in the method Nielsen (1986) used for determining the bottom (reference) concentration. Indeed, Equations 190 and $186\left(A_{c R, w}=A_{c R, c}\right)$ still yield the best results. Compared to the results with a current, however, the range of values for $d *$ in the data 
set with current only was wider ( $d *$ from 1.0 to 18 ), and the grain size distribution of the data is different compared to the data sets for current (Figure 69) where $d *<5$ for 40 percent of the data against 95 percent for the data set with waves only. This may explain the difference compared to the results for the current, and especially the difference observed using Equation 190 with $A_{c R}=510^{-4}$ (overestimation for the current data set and underestimation for the wave data set). Also, the effect of the dimensionless grain size appears to still be significant. As an alternative, Camenen and Larson (2007) proposed a relationship for $A_{c R}$ similar to Nielsen (1986), but with different coefficient values:
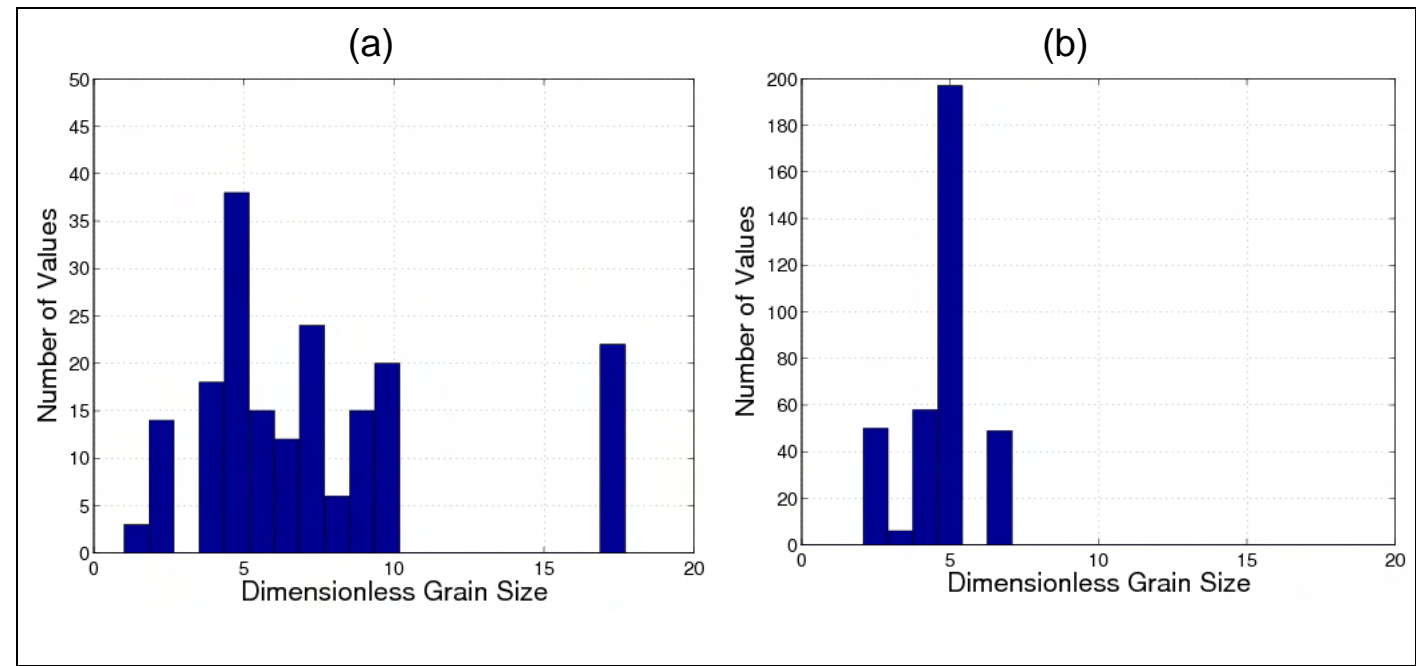

Figure 69. Histograms of grain-size distribution for current data set (a) and wave data set (b).

The equation proposed by Camenen and Larson (2007) yields similar results to Equation 186 (see Table 30). However, large uncertainties arise from estimation of the ripple characteristics and the associated induced Nikuradse roughness. Thus, in Table 30 are the results from using two different methods to estimate the Nikuradse roughness displayed: the first method employs the expressions by Van Rijn (1993) for both ripple characteristics and roughness computation, whereas the second method uses the Soulsby and Whitehouse (2005a, b) relationships for the ripple characteristics and the equations by Kim (2004) for the roughness computation. As shown in Table 30, the method selected to estimate the Nikuradse roughness affects the results more significantly than using Equation 186 or the equation proposed by Camenen and Larson (2007). In the following, only Equation 186 together with the Van Rijn (1993) method to estimate the Nikuradse roughness will be applied. 
A reason for the larger scatter is the uncertainty concerning the bed forms (measured or calculated using Van Rijn (1993) relationships) and the total shear stress calculation (using the Nielsen method with Equation 189, or the classical method estimating the Nikuradse roughness due to ripples from Equation 141). The coefficient $A_{c R}$ is observed to be a function of the ripple height $H_{r}$, or more specifically of the Nikuradse roughness ratio $k_{s} / d_{s}$ (where $d_{s}$ is the median grain size of the suspended sediments, Figure 70). The Madsen (1993) formula (as well as Equation 190 with $A_{c R}=510^{-4}$ ) again shows reasonable results because it is not as sensitive to $d *$ and $\theta$.

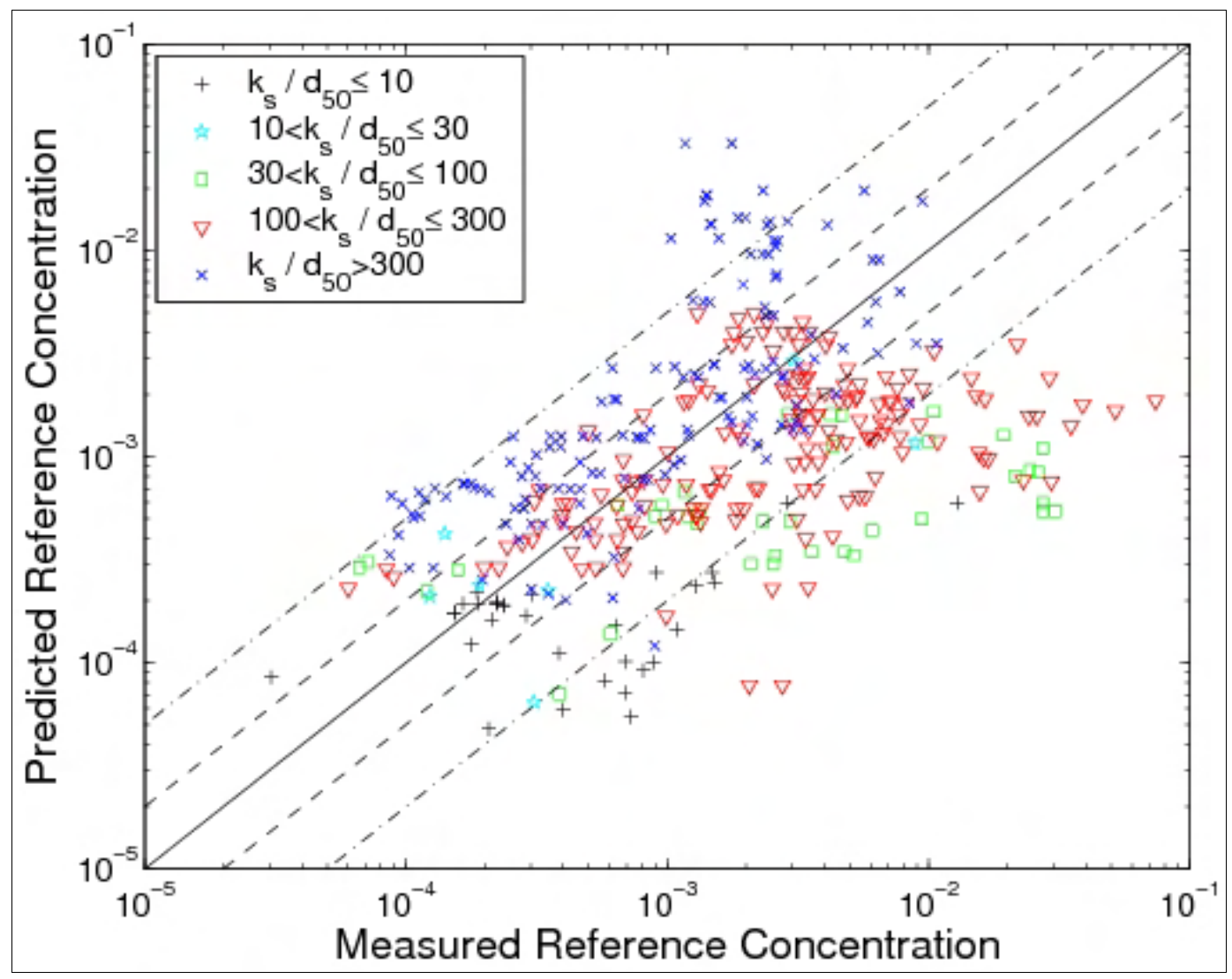

Figure 70. Reference concentration $c_{R}$ estimated from compiled data (see Table 19) versus $c_{R}$ calculated with Equation 190 and 186 with roughness ratio emphasized.

Figure 70 plots the reference concentration $c_{R}$ estimated from the compiled data (see Table 19 for cases where waves, i.e., $U_{c}<0.05 \mathrm{~m} / \mathrm{sec}$ ). These values are plotted versus $c_{R}$ calculated with Equations 190 and 186. The roughness ratio is emphasized, indicating that the calculation of the total shear stress produces large uncertainty (assuming that the reference concentration $c_{R}$ should not be a function of the ripple height or the 
roughness ratio but only of the total shear stress). It seems that the more overestimated or underestimated the reference concentration is, the larger or smaller the roughness ratio is, respectively.

Figure 71 plots the roughness ratio $k_{s} / d_{50}$ versus the total Shields parameter plotted with the ripple height emphasized. It appears that the Nikuradse roughness and Shields parameter tend to be excessively large $\left(\theta_{w}>10\right)$ for large ripple heights (the Van Rijn (1984c) method was used for the computations). An accurate prediction of the total shear stress thus appears to be a key factor for accurate prediction of the reference concentration.

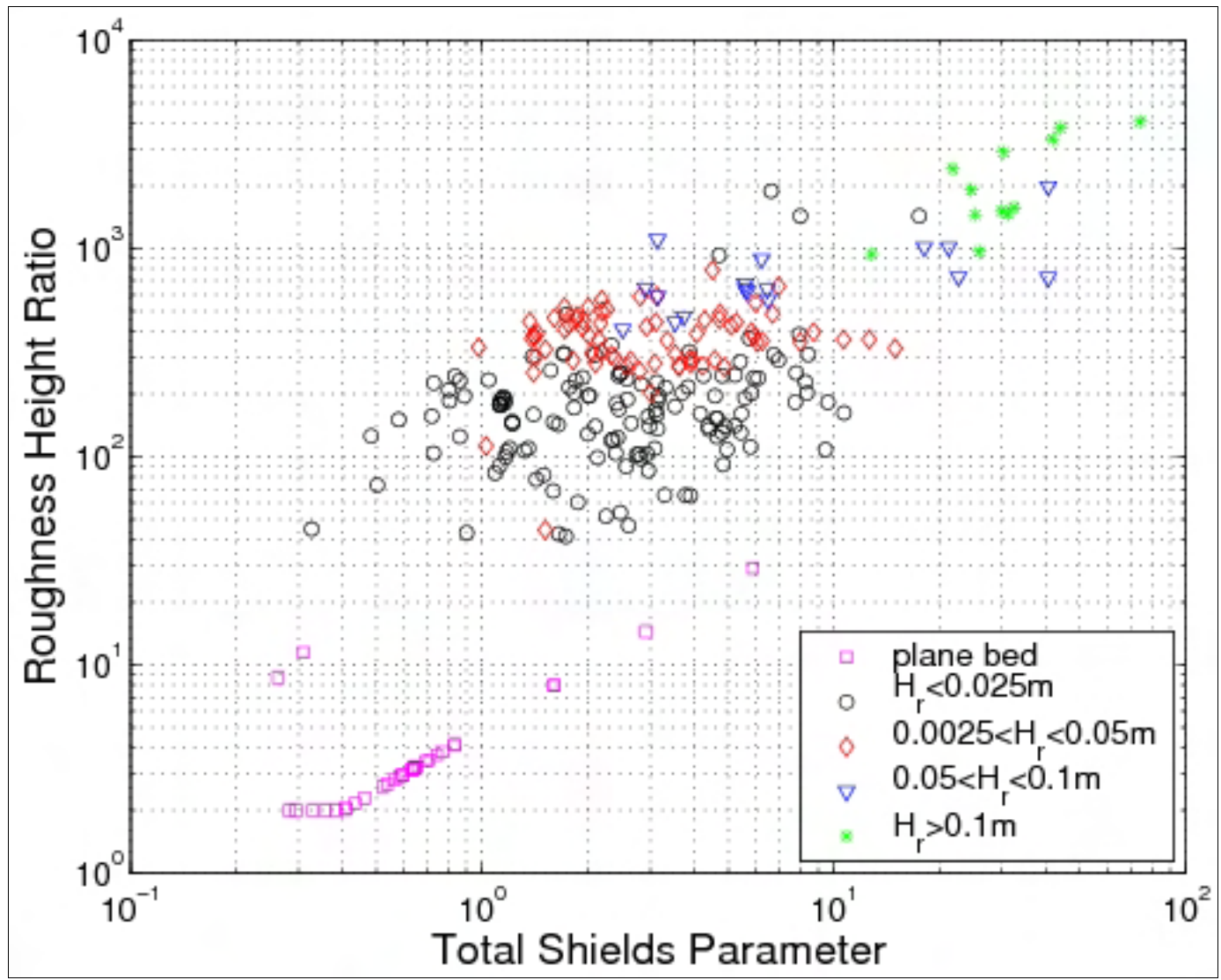

Figure 71 . Estimated roughness ratio $k_{s} / d_{50}$ versus total Shields parameter with ripple height emphasized.

Figure 72 plots the reference concentration $c_{R}$ estimated from the compiled data where waves were dominant $\left(U_{c}<0.05 \mathrm{~m} / \mathrm{sec}\right)$ plotted versus $c_{R}$ calculated with Equations 190 and 186 for all the data sets. It appears that the results do not depend on the specific data set except for the Nielsen 


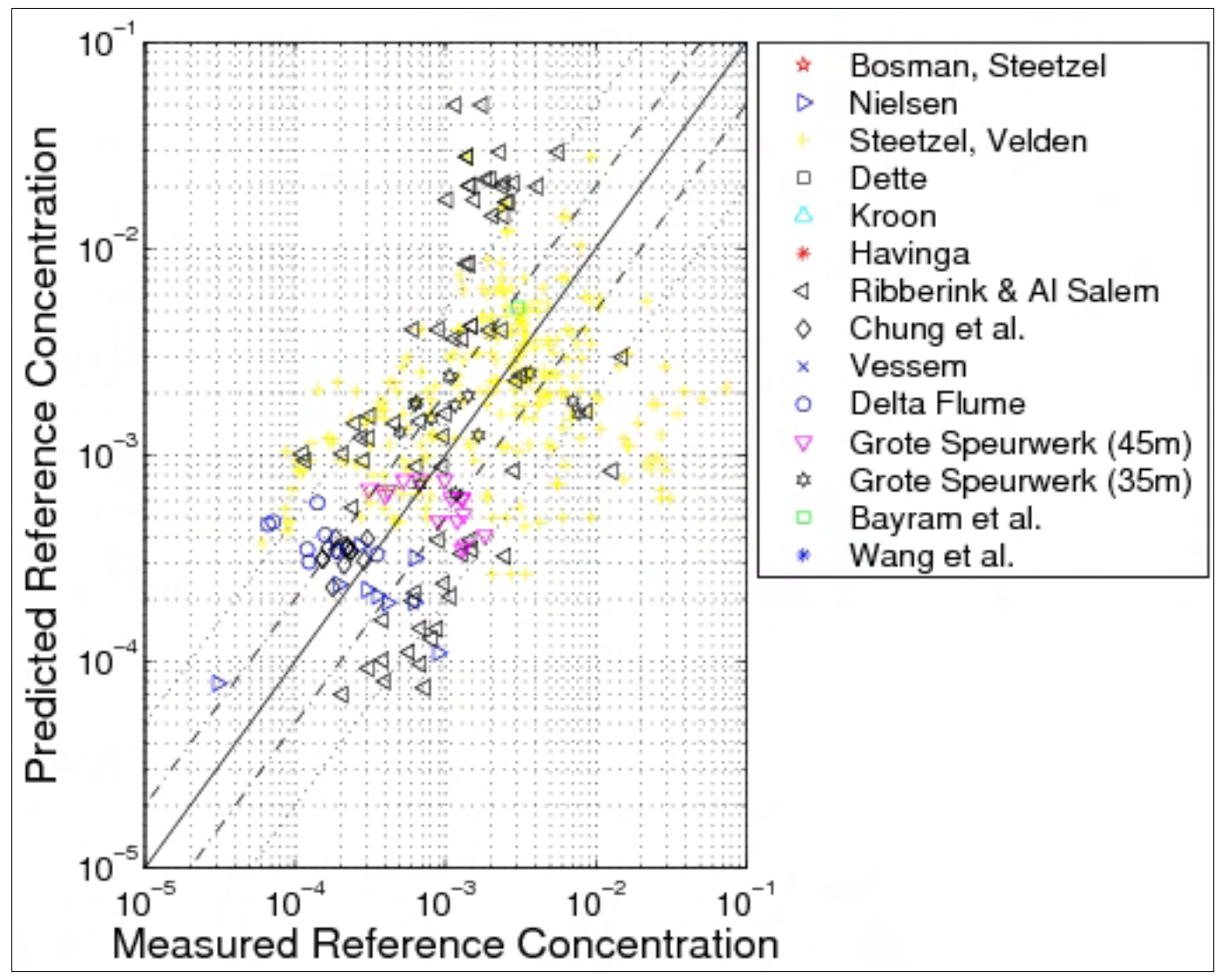

Figure 72. Reference concentration $c_{R}$ estimated from data compiled for waves only versus $c_{R}$ calculated with Equations 190 and 186.

(1984) data, which are generally overestimated and the Steetzel (1984) and Van der Velden (1986) data which are underestimated. The larger discrepancy observed for the Ribberink and Al Salem (1994) data set may be due to the calculation of the total shear stress, which yields underestimations for the plane cases and overestimations for the ripple cases.

\section{Wave-current interaction}

For situations where the wave-current interaction is significant, the intuitive Shields parameters to use in Equation 185 are $\theta_{T}=\theta_{c w, m}$ and $\theta_{M}=\theta_{c w}$ :

$$
c_{R}=A_{c R, c w} \theta_{c w, m} \exp \left(-4.5 \frac{\theta_{c r}}{\theta_{c w}}\right)
$$


There are several ways of estimating the shear stresses needed in Equation 192. To simplify the calculations, assuming a sinusoidal orbital wave velocity variation, the mean and maximum Shields parameter due to wave and current interaction may be obtained through vector addition, respectively.

$$
\begin{gathered}
\theta_{c w, m}=\sqrt{\theta_{c}{ }^{2}+\theta_{w, m}^{2}+2 \theta_{c} \theta_{w, m} \cos \varphi} \\
\theta_{c w}=\sqrt{\theta_{c}{ }^{2}+\theta_{w}^{2}+2 \theta_{c} \theta_{w} \cos \varphi}
\end{gathered}
$$

in which $\varphi$ is the angle between the wave and current directions. The mean Shields parameter due to wave and current interaction may also be estimated as follows:

$$
\theta_{c w, m}=\frac{1}{2} \frac{f_{c w}}{(s-1) g d_{50}}\left[\frac{1}{T_{w}} \int_{0}^{T w}\left(U_{c} \cos \varphi+u_{w}(t)\right)^{2} d t+\left(U_{c} \sin \varphi\right)^{2}\right]
$$

The friction coefficient $f_{c w}$ is assumed to be constant and it is calculated using the Grant and Madsen (1979) formula, i.e., $f_{c w}=f_{c} X_{v}+f_{w}\left(1-X_{v}\right)$ where $X_{v}=\left|U_{c}\right| /\left(\left|U_{c}\right|+U_{w}\right)$. Furthermore, using a linear weighting between the current-related Shields parameter and the mean wave-related Shields parameter yields:

$$
\theta_{c w, m}=X_{v} \theta_{c}+\left(1-X_{v}\right) \theta_{w, m}
$$

Finally, Soulsby (1997, pp.87-95) proposed a method for estimating the wave-current mean and maximum shear stresses.

The percentage of values obtained within a factor 2 or 5 , as well as the mean value and the standard deviation of the ratio $f\left(c_{R}\right)=$ $\log \left(c_{R, p r e d} / c_{R \text {,meas }}\right)$, are presented in Table 31 using the investigated formulas and Equation 192 with the two expressions found for $A_{c R}$. The Nielsen 1986) formula presents better results compared to the case with waves only, perhaps because the shear stress $\theta_{r}$ does not take into account the effect of the current well. On the other hand, the Madsen (1993) formula does not give as much scatter, but it generally overestimates the measurements. The new formula with $\theta_{T}=\theta_{c w, m}$ (using Equation 186 to obtain $c_{R}$ ) still yields the best results among the studied formulas. 
Table 31. Prediction of reference concentration using compiled data set with wave-current interaction.

\begin{tabular}{|l|l|l|l|l|}
\hline Author(s) & $P \times 2(\%)$ & $P \times 5(\%)$ & Mean $\left(f\left(c_{R}\right)\right)$ & Std $\left(f\left(c_{R}\right)\right)$ \\
\hline Nielsen & 43 & 66 & 0.29 & 0.73 \\
\hline Madsen & 02 & 16 & 1.04 & 0.43 \\
\hline Equations, 192 and 186 & 50 & 85 & 0.20 & 0.45 \\
\hline Equations 192 and 191 & 02 & 13 & 1.14 & 0.42 \\
\hline Equations, 192 with $A_{c r}=510^{-4}$ & 52 & 90 & -0.11 & 0.43 \\
\hline
\end{tabular}

As for the cases with waves only, Equation 191 induces large overestimation of the results (factor of 10). Surprisingly, using Equation 192 with $A_{c r}=510^{-4}$ produces much better estimations. This is mainly because the constant value for $A_{c r}$ is typically smaller than varying the value (Equation 186) and compensates for the larger Shields parameter values observed in case of wave-current interaction. Indeed, it appears that the computation of the mean wave and current Shields parameter $\theta_{c w, m}$ significantly influences the results. If Equation 192 tends to underestimate the results in case of waves only (cf. Table 30), it tends to overestimate the results for a wave-current interaction.

The different methods for computing the mean shear stress (Equations 193, 194, and 195 together with the Soulsby (1997) method) were also compared. The "addition" method (Equation 193) and the linear weighting method (Equation 195) yield similar results, whereas the integration method appears to be more sensitive to the current and yields larger values if $U_{c}$ is not negligible. The Soulsby (1997) method yields much smaller values because the proposed definition of the mean Shields parameter is different. This definition corresponds to a time-averaged Shields parameter with the direction included. Thus, for a pure sinusoidal wave without current, it yields $\theta_{c w, m}=0$, whereas the proposed method (time-averaging of the absolute value of the Shields parameter) yields $\theta_{c w, m}=1 / 2 \theta_{c w}$.

Figure 73 plots the calculated reference concentrations with Equations 192, 193, and 186 against the estimated reference concentration from the data with the absolute mean current and the roughness ratio emphasized. It appears that the effect of the mean current or the roughness ratio is not significant for the wave-current interaction. 


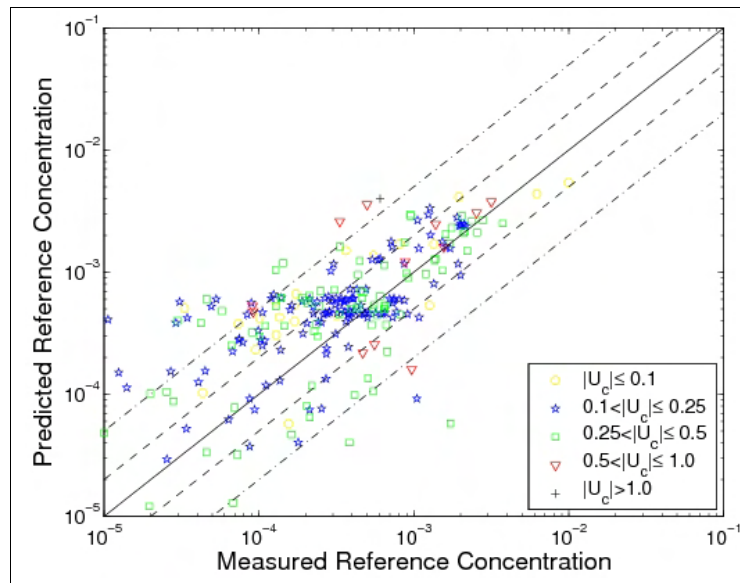

(a)

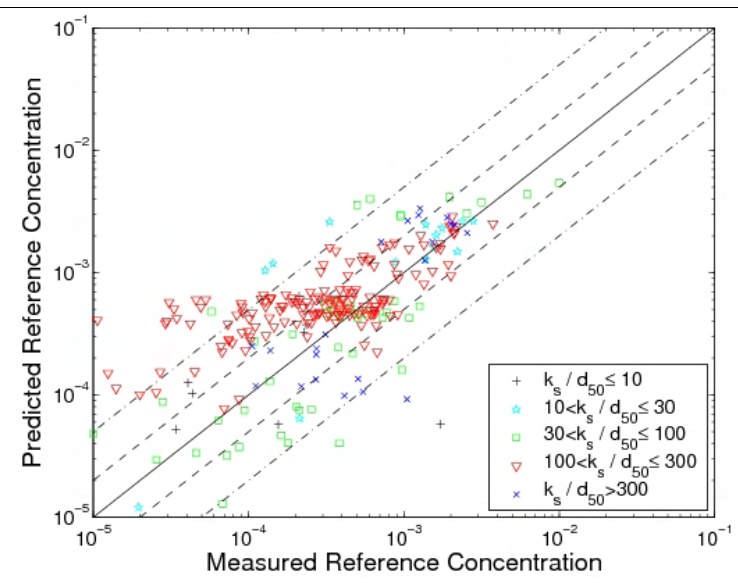

(b)

Figure 73. Reference concentration $c_{R}$ estimated from compiled data set with wave-current interaction versus $C_{R}$ calculated with Equations 192, 193, and 186 with (a) absolute mean current $\left|U_{c}\right|$ or $(b)$ roughness ratio $k_{s} / d_{50}$, emphasized.

The new relationship for $c_{R}$ significantly improves calculated estimates as compared to the other formulas: more than 45 percent of the data are correctly predicted within a factor 2 and more than 75 percent within a factor 5. Figure 74 plots the reference concentration $c_{R}$ estimated from the compiled data set where both current and waves are present versus $c_{R}$ calculated with Equations 192, 193, and 186. The results appear to vary depending on the specific data set: the Vessem (Van Rijn et al. 2001) and the Bayram et al. (2001) data sets are often overestimated, whereas the Nielsen (1984) and Kroon (1991) data sets are generally underestimated. It should be noted that the ripple height for the Vessem data set was estimated to be $H_{r}=0.05 \mathrm{~m}$ for all the cases.

\section{Cases with breaking waves}

Negligible effect of breaking waves on bed reference concentration. As a first approach, it is assumed that wave breaking does not affect the reference concentration, but only the sediment diffusivity. The turbulence induced by the breakers is expected to occur in the upper part of the water column; thus, it should not influence the bottom concentration significantly.

The data sets presented in Table 33 involve many experimental cases where breaking waves occurred. These data sets are from flume experiments (Bosman 1982; Steetzel 1985; Dette and Uliczka 1986; Peters 2000; 


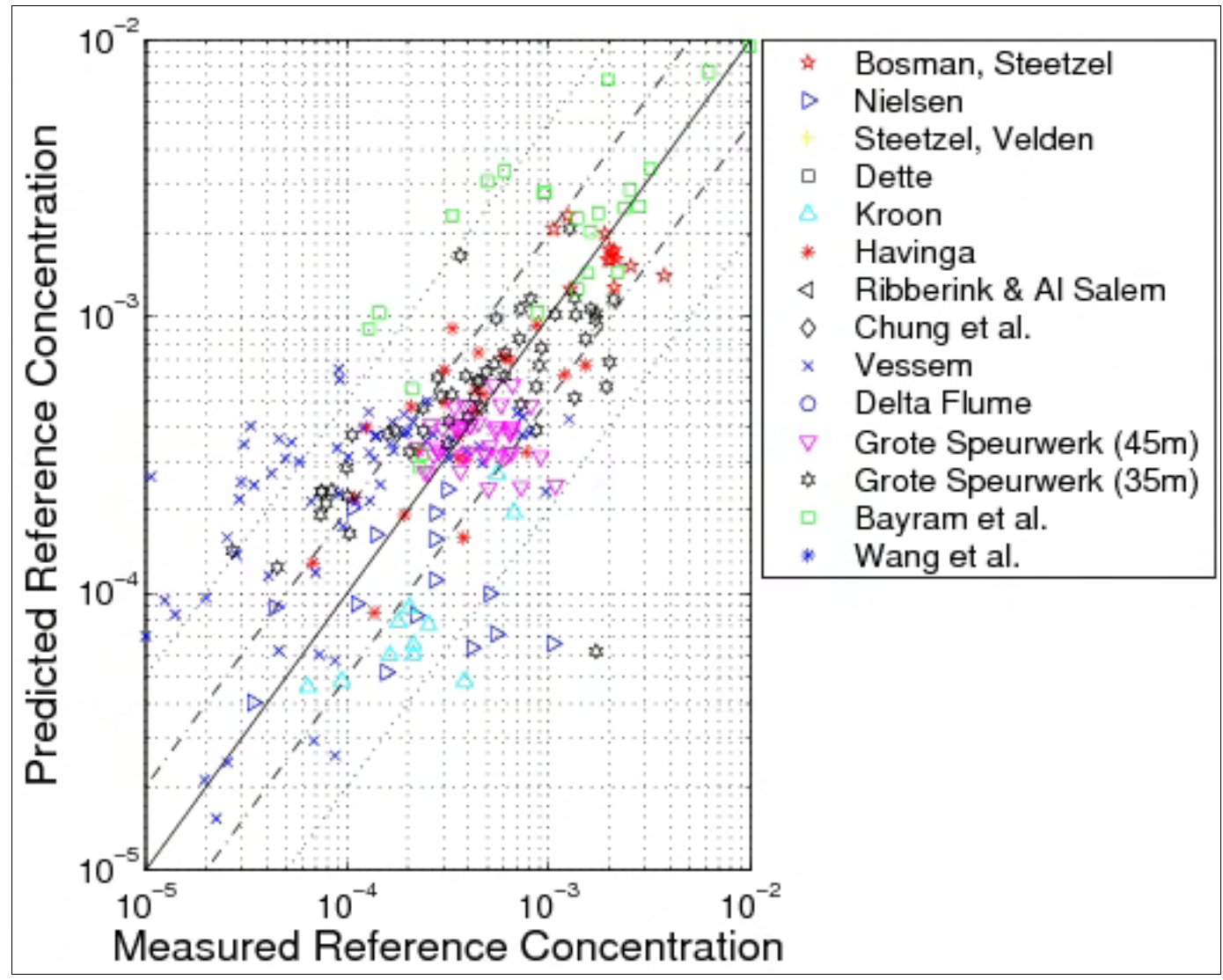

Figure 74. Reference concentration $c_{R}$ estimated from data compiled with wave-current interaction versus $C_{R}$ calculated with Equations 192, 193, and 186.

SEDMOC Van Rijn et al. 2001) with the Grote Speurwerk (35 m) and the Delta Flume), basin experiments (Havinga 1992; Wang et al. 2002), and from field experiments (Nielsen 1984; Kroon 1991; Bayram et al. 2001). Because the Peters (2000) data did not include the actual concentration profiles collected, but only the exponential fits to the data, comparisons for this data set are made separately.

Table 32 presents the calculations depending on the chosen formula. Although the results are scattered, Equation 192 together with Equation 186 present the best results among the studied formulas with about 45 percent of the data correctly predicted within a factor 2 and 75 percent within a factor 5 . The use of the constant $A_{c R}=510^{-4}$ does not yield results as good as found for the wave and current interaction. Also, the use of Equations 193, 194 or 195 does not significantly change the results, because the waves are dominant. 
Table 32. Prediction of reference concentration using compiled data set with breaking waves.

\begin{tabular}{|l|l|l|l|l|l|}
\hline Author(s) & Px2 (\%) & Px5 (\%) & Mean $\left(f\left(c_{R}\right)\right)$ & Std $\left(f\left(c_{R}\right)\right)$ \\
\hline \multicolumn{5}{|c|}{ All Data Except Peters (2000) } \\
\hline Nielsen & 22 & 51 & 0.66 & 0.77 \\
\hline Madsen & 15 & 54 & 0.74 & 0.52 \\
\hline Equations 192, 193, and 186 & 44 & 77 & -0.02 & 0.57 \\
\hline Equations 192, 193 with $A_{C R}=510^{-4}$ & 40 & 77 & -0.26 & 0.50 \\
\hline \multicolumn{5}{|l|}{ Peters (2000) Data } \\
\hline Nielsen & 36 & 75 & 0.31 & 0.48 \\
\hline Madsen & 44 & 89 & 0.34 & 0.28 \\
\hline Equations 192, 193, and 186 & 23 & 52 & -0.65 & 0.39 \\
\hline Equations 192, 193 with $A_{C R}=510^{-4}$ & 08 & 45 & -0.73 & 0.30 \\
\hline
\end{tabular}

Table 33. Data summary for suspended sediment experiments under oscillatory flows (GS35 and GS45 correspond to "Grote Speurwerk" with length of 35 and $45 \mathrm{~m}$, respectively).

\begin{tabular}{|c|c|c|c|c|c|c|c|c|c|}
\hline Author(s) & No. Exp. & $d_{50}(\mathrm{~m})$ & $h(\mathrm{~m})$ & $U_{c}(\mathrm{~m} / \mathrm{sec})$ & $U(\mathrm{z})$ meas. & \begin{tabular}{|l}
$U_{w^{1}}$ \\
$(\mathrm{~m} / \mathrm{sec})$
\end{tabular} & $T_{w^{1}}(\mathrm{sec})$ & $H_{r}(\mathrm{~m})$ & $L_{r}(\mathrm{~m})$ \\
\hline $\begin{array}{l}\text { Bosman (1982) and } \\
\text { Steetzel (1985) }\end{array}$ & 16 & 0.10 & $0.1-0.65$ & $\begin{array}{l}0.10- \\
0.32\end{array}$ & $3-4 \mathrm{pts}$ & $\begin{array}{l}0.13- \\
0.30\end{array}$ & $1.4-2.0$ & $-.01-0.03$ & 0.08 \\
\hline Nielsen (1984) & 43 & $\begin{array}{l}0.11- \\
0.62\end{array}$ & $0.8-1.8$ & $0-0.54$ & $5-7 \mathrm{pts}$ & $\begin{array}{l}0.28- \\
0.80\end{array}$ & $5.3-14.4$ & $0^{2}-0.20$ & $0^{2}-1.5$ \\
\hline Kroon (1991) & 62 & $\begin{array}{l}0.30- \\
0.47\end{array}$ & $0.4-1.5$ & $\begin{array}{l}-0.55- \\
0.97\end{array}$ & $3-5 \mathrm{pts}$ & $\begin{array}{l}0.20- \\
0.91\end{array}$ & 3.1 - 12.6 & $\begin{array}{l}0.005- \\
0.05\end{array}$ & $\begin{array}{l}0.15- \\
0.75\end{array}$ \\
\hline Havinga (1992) & 28 & 0.10 & $\begin{array}{l}0.40- \\
0.43\end{array}$ & $\begin{array}{l}0.10- \\
0.32\end{array}$ & $10 \mathrm{pts}$ & $0-0.80$ & $2.1-2.3$ & -3 & $-{ }^{3}$ \\
\hline Chung et al. (2000) & 14 & $\begin{array}{l}0.16- \\
0.33\end{array}$ & $3.5-4.5$ & $\begin{array}{l}-0.04-- \\
0.02\end{array}$ & $5 \mathrm{pts}$ & $\begin{array}{l}0.56- \\
0.67\end{array}$ & $6.6-7.1$ & $\begin{array}{l}0.03- \\
0.05\end{array}$ & $\begin{array}{l}0.25- \\
0.75\end{array}$ \\
\hline $\begin{array}{l}\text { Gailani and Smith } \\
\text { (2000) }\end{array}$ & 818 & 0.22 & $\begin{array}{l}16.6- \\
19.5\end{array}$ & $0-0.88$ & $1 \mathrm{pt}$ & $\begin{array}{l}0.03- \\
1.49\end{array}$ & $4.8-21.3$ & $-{ }^{3}$ & $-{ }^{3}$ \\
\hline $\begin{array}{l}\text { SEDMOC data set } \\
\text { (Van Rijn et al. } \\
\text { 2001) -Vessem- }\end{array}$ & 70 & 0.15 & $0.7-4.0$ & $\begin{array}{l}0.05- \\
0.65\end{array}$ & $1 \mathrm{pt}$ & $\begin{array}{l}0.02- \\
0.40\end{array}$ & $2.0-3.2$ & 0.05 & $-{ }^{3}$ \\
\hline $\begin{array}{l}\text { SEDMOC data set } \\
\text { (Van Rijn et al. } \\
\text { 2001) -GS45- }\end{array}$ & 19 & $\begin{array}{l}0.15- \\
0.29\end{array}$ & $\begin{array}{l}0.49- \\
0.55\end{array}$ & $\begin{array}{l}0.16- \\
0.35\end{array}$ & 2 - 3 pts & $\begin{array}{l}0.14- \\
0.60\end{array}$ & $2.4-2.8$ & -3 & $-{ }^{3}$ \\
\hline $\begin{array}{l}\text { SEDMOC data set } \\
\text { (Van Rijn et al. } \\
\text { 2001) - GS35- }\end{array}$ & 58 & $\begin{array}{l}0.10- \\
0.22\end{array}$ & $\begin{array}{l}0.29- \\
0.60\end{array}$ & $\begin{array}{l}0.07- \\
0.45\end{array}$ & $9-12$ pts & $0.17-0.55$ & $1.2-2.7$ & $\begin{array}{l}0.002- \\
0.029\end{array}$ & $\begin{array}{l}0.006- \\
0.20\end{array}$ \\
\hline $\begin{array}{l}\text { Bayram et al. } \\
\text { (2001) }\end{array}$ & 66 & $\begin{array}{l}0.18- \\
0.20\end{array}$ & $1.2-8.6$ & $\begin{array}{l}0.04- \\
1.32\end{array}$ & $3 \mathrm{pts}$ & $0.71-2.13$ & $8.0-12.8$ & -3 & -3 \\
\hline Wang et al. (2002) & 14 & 0.22 & $\begin{array}{l}0.10- \\
0.40\end{array}$ & $0-0.18$ & $6-9$ pts & $\begin{array}{l}0.27- \\
0.45\end{array}$ & $1.5,3.0$ & -3 & $-{ }^{3}$ \\
\hline
\end{tabular}


For the data sets with breaking waves, the sensitivity to grain size appears again not to be as significant as for the steady current data (Figure 75), because the data with larger grain size tend to be underestimated. However, the constant value $A_{c R}=510^{-4}$ presents results that are not as good as previously found, with larger underestimation. In case of the Peters (2000) data, this constant value yields a large underestimation, similar to Equation 186. This data set, however, seems to be unusually insensitive to the main parameters (e.g., Shields parameter, grain size). Thus, $c_{R}$ varies from $1.310^{-3}$ to $5.910^{-3}$ for this data set, whereas $\theta_{c w, m}$ varies from 0.3 to 2 ( $U_{w}$ varies from 0.5 to 1.25 ) and $d *$ varies from 3 to 8.
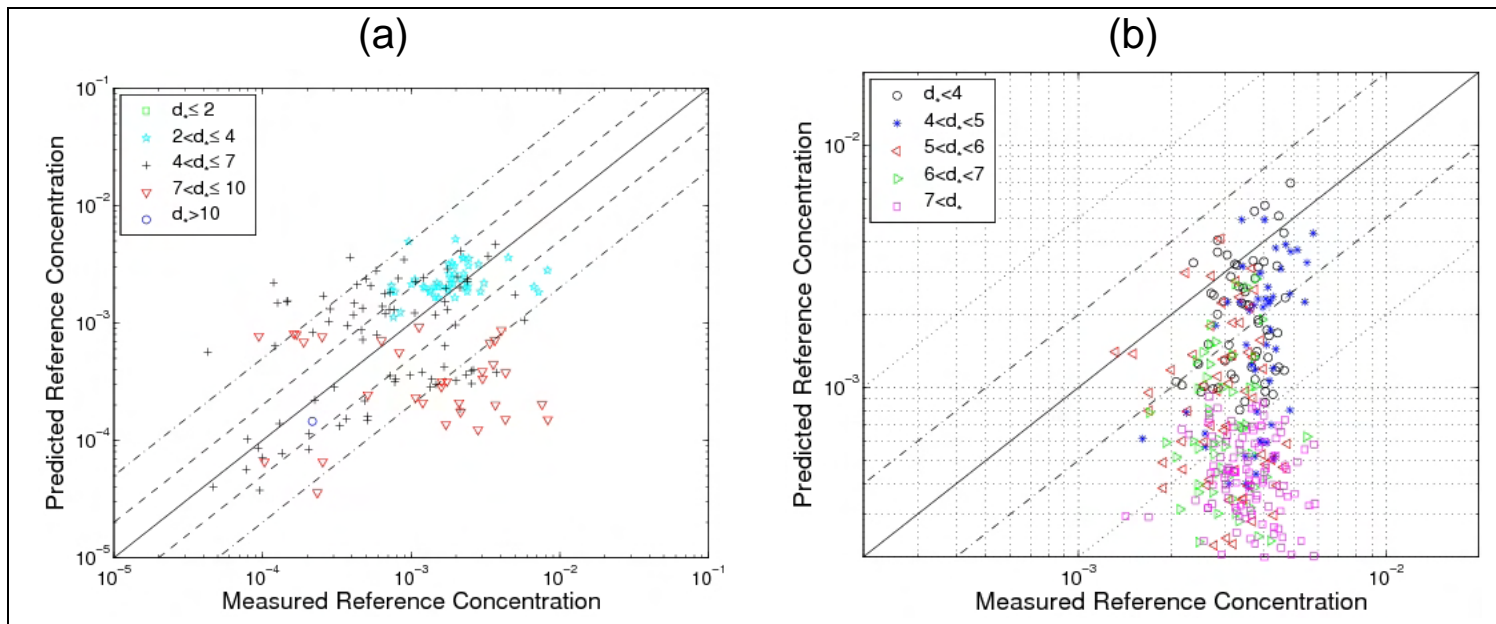

Figure 75. Reference concentration $c_{R}$ estimated from data compiled with breaking waves (a) excluding data from Peters (2000), and (b) using data set from Peters only, versus $C_{R}$ calculated with Equations 192, 193, and 186.

Using the constant value $c_{R}=310^{-3}$ gives a prediction of 99 percent with an error of a factor 2 allowed.

Influence of Irribaren parameter. For plunging breakers, the generated turbulent jet may penetrate to the bottom and influence the reference concentration. To study this effect, some specific data sets where beach profiles are known were examined (Table 24 in the "Experimental data with breaking waves" section). Figure 76 plots the ratio between the estimated reference concentration and the predicted reference concentration using Equations 192 and 186 plotted against the Irribaren parameter $\xi_{\infty}$. For the field experiments (the Bayram et al. (2001) and Voulgaris and Collins (2000) data sets), it seems that $c_{R, \text { meas }} / c_{R, \text { pred }}$ is an increasing function of the Irribaren parameter. If $\xi_{\infty}<0.2$ (spilling breakers), the proposed relationship tends to overestimate the measurements, whereas for $\xi_{\infty}>0.2$ 


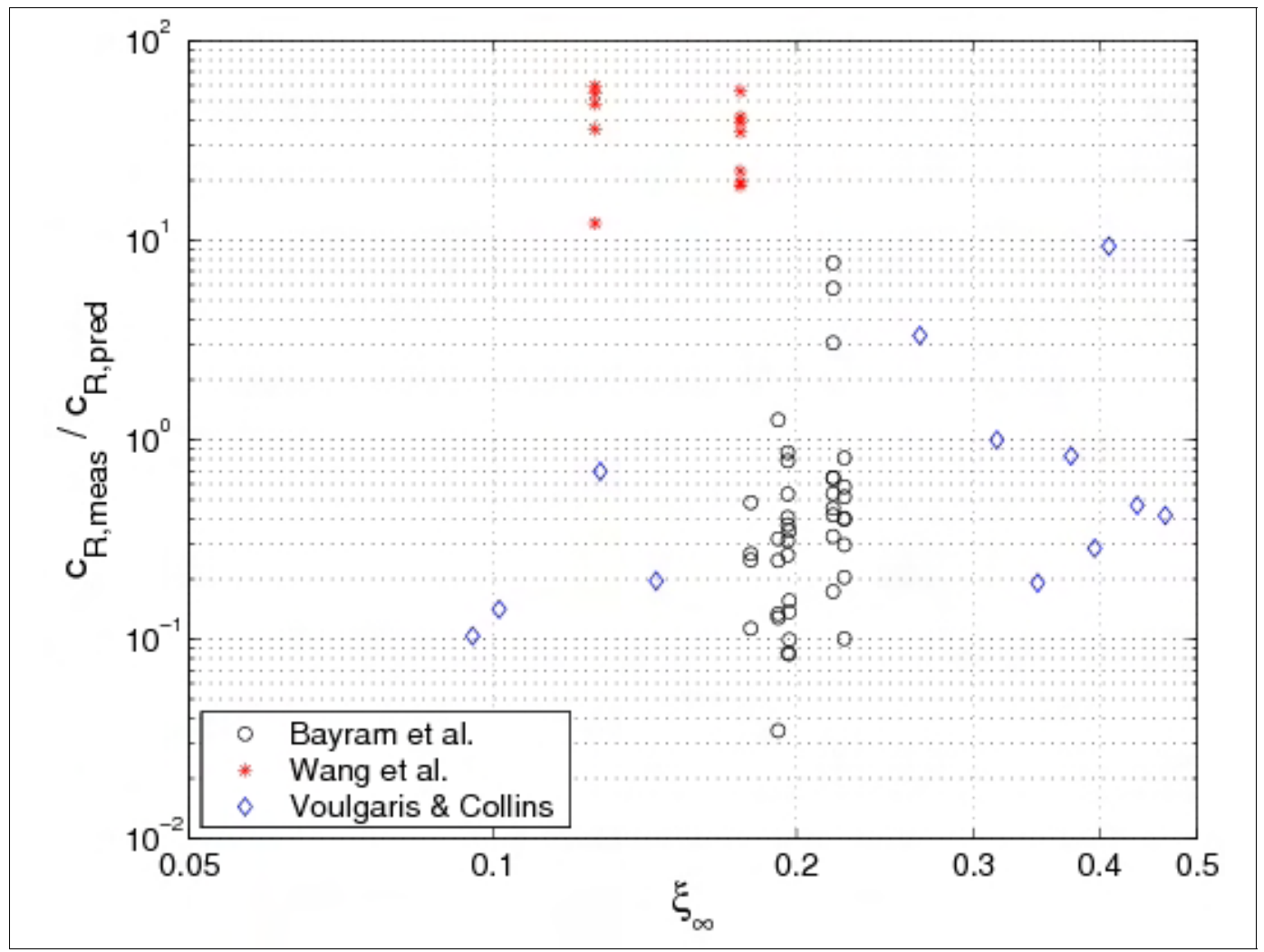

Figure 76. Ratio between estimated reference concentration and predicted reference concentration from Equations 192 and 186 as function of Irribaren parameter $\xi_{\infty}$ using data sets from Table 24.

(plunging breakers), the predictions are slightly better. In case of the Wang et al. (2002) data, the equations yield underestimations that decrease with $\xi_{\infty}$. Nevertheless, from all these experimental data sets, there is an indication that plunging breakers may increase the reference concentration. Viewing the results for the Kroon (1991) and Wang et al. (2002) data sets (Figure 77), it appears that the reference concentration is underestimated for the larger observed reference concentrations where strong energy dissipation due to plunging breaking waves was present.

An empirical equation may be introduced to take into account the effects of breaking waves (especially plunging waves) on the reference concentration by introducing a stirring coefficient:

$$
C_{R b}=1.0+\tanh \left[50\left(\xi_{\infty}-0.15\right)\right] \text { if } \xi_{\infty}>0.15
$$

where the reference concentration should be multiplied with $C_{R b}$. This equation is only valid for $\xi_{\infty}<3.0$, i.e., for spilling and plunging breakers. 


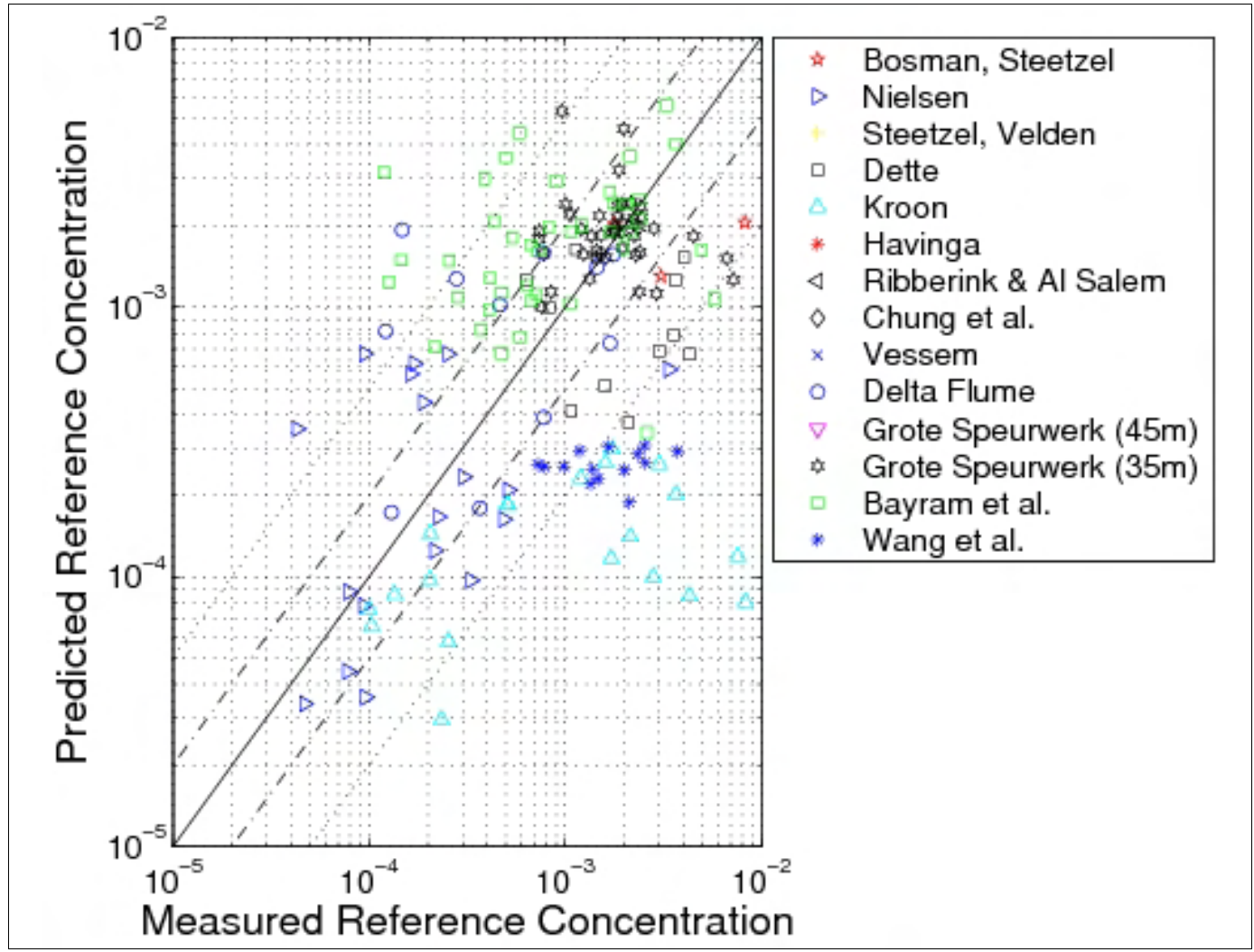

Figure 77. Reference concentration $c_{R}$ estimated from data compiled with breaking waves (see Table 33) versus $c_{R}$ calculated with Equations 192, 186, and 196.

Equation 196 was employed for all the data where waves were breaking (the offshore wave conditions were estimated if not available), but little net improvement in agreement was observed. Thus, there is a need for new measurements to estimate the effect of the breakers on the bottom concentration.

Viewing the different data sets (Figure 77), the bottom reference concentration is overall correctly estimated, but it does depend on the particular data set. The data from Dette and Uliczka (1986), Kroon (1991), and Wang et al. (2002) are typically underestimated, whereas the data from the Delta Flume and Bayram et al. (2001) are often overestimated.

\section{Suspended load transport}

The unsteady depth-averaged volumetric suspended load transport $q_{s s}$ may be calculated by averaging the product between the suspended sediment concentration $c$ and the velocity $u$ over the water depth. 


\section{Existing formulas for suspended load under wave-current interaction}

Many formulas have been proposed to estimate the suspended load. This section presents selected popular ones, discussing underlying hypotheses, and key background studies.

The Bijker formula. To calculate the suspended load, Bijker (1967) assumed that bed load occurred in a bottom layer having a thickness equal to the roughness and with a constant concentration over the thickness:

$$
c_{b}=\frac{q_{s b}}{6.34 \sqrt{\tau_{c} / \rho} k_{s, c w}}
$$

where $k_{s, c w}$ is the bottom roughness due to wave and current interaction. The concentration distribution is obtained from the following equation:

$$
c(z)=c_{b}\left(\frac{k_{s, c w}}{h-k_{s, c w}} \frac{h-z}{z}\right)^{\frac{W_{s}}{\kappa} \sqrt{\frac{\rho}{\tau_{c w}}}}
$$

where $\tau_{c w}$ is total shear stress for interaction between current and waves computed following Bijker's method. Integrating over the vertical from $z=k_{s, c w}$ to $z=h$, the total suspended load is determined as:

$$
q_{s s}=1.83 q_{s b}\left(I_{1} \ln \left[\frac{33 h}{\delta_{c}}\right]+I_{2}\right)
$$

where $q_{s b}$ and $q_{s s}$ is the sediment volume fluxes for bed load and suspended load, respectively, $I_{1}$ and $I_{2}$ are the Einstein integrals (suspended load), and $\delta_{c}=k_{s, c w} / h$ is the dimensionless thickness of the bed-load layer.

The Einstein integrals for the suspended load are given by the following equations:

$$
\begin{aligned}
& I_{1}=\int_{\delta}^{1}\left(\frac{1-y}{y}\right)^{A_{E}} d y, \\
& I_{2}=\int_{\delta}^{1}\left(\frac{1-y}{y}\right)^{A_{E}} \ln y d y
\end{aligned}
$$


where $A_{E}=W_{s} / \kappa\left(\tau_{c w} / \rho\right)^{0.5}$ is a function determining the suspension rate.

The Engelund and Hansen formula. The total load was expressed by Engelund and Hansen (1972) as:

$$
q_{s}=0.05 \sqrt{\frac{(s-1)}{g}} K_{s}^{2} h^{1 / 3} d_{50}^{3 / 2} \theta_{E H}^{5 / 2}
$$

where $K_{s}=\sqrt{2 g / f_{c}} h^{-1 / 6}$ is the Strickler parameter, and $\theta_{E H}$ a modified Shields parameter dependent on the transport regimes:

$$
\begin{gathered}
\theta_{E H}=0 \text { if } \theta \leq c_{1} \\
\theta_{E H}=2.5\left(\theta-c_{1}\right)^{0.5} \text { if } c_{1}<\theta \leq c_{2} \\
\theta_{E H}=1.065 \theta^{0.176} \text { if } c_{2}<\theta \leq c_{3} \\
\theta_{E H}=\theta \text { if } c_{3}<\theta
\end{gathered}
$$

where $c_{1}=0.06, c_{2}=0.384$, and $c_{3}=1.08$.

The Van Rijn formula. Time-averaged concentration is computed by solving the equation for concentration over depth:

$$
\frac{d c}{d z}=-\frac{(1-c)^{5} c W_{s}}{\varepsilon_{s, c w}}
$$

where $c(z)$ is the mean volume concentration (time-averaged) at elevation $z,(1-c)^{5}$ corresponds to the decrease in the settling velocity for large concentrations, and $\varepsilon_{s, c w}$ is the mixing coefficient for the wave-current interaction.

Then, sediment flux is integrated over the water depth from the reference level $z_{a}=\max \left(k_{s, t c}, k_{s, t w}\right)\left(k_{s, t c}\right.$ and $k_{s, t w}$ are the total roughness values due to current and waves, respectively) to $h$. The parameters $\varepsilon_{s, c w}, c_{a}$, and $u(\bar{z})$ are computed following the equations given by Van Rijn (1984b, 1989, 1993; see "Equilibrium profile for suspended sediment" section). 
The Bailard formula. Using similar hypothesis as for the bed-load transport, Bailard (1981) proposed an expression for the suspended load:

$$
\vec{q}_{s}=\frac{0.5 f_{c w}}{g(s-1)} \frac{\varepsilon_{s}}{W_{s}}<|\vec{u}|^{3} \vec{u}>
$$

where $\varepsilon_{s}$ is the suspended load efficiency and $<>$ corresponds to an average over several wave periods.

\section{A simple formula}

The traditional approach was applied in this study of calculating the vertical distributions of suspended sediment concentration and velocity, after which the product between these two quantities is integrated through the vertical to obtain the suspended load transport. Basically, the present formulation closely follows the simplified approach by Madsen et al. (2003).

If the current velocity is constant over depth, the suspended load transport $\left(q_{s s}\right)$ is obtained from:

$$
q_{s s}=\int_{z_{R}}^{h} c(z) u(z) d z=U_{c} \int_{z_{R}}^{h} c(z) d z
$$

where $z_{R}$ is the reference level separating bed load and suspended load, $h$ the water depth, $c$ the concentration, $u$ the horizontal velocity (varying through the vertical in the general case), $z$ the vertical coordinate, and $U_{c}$ the mean horizontal velocity. In determining $q_{s s}$, the vertical variation in $u$ will be neglected.

Thus, assuming an exponential concentration profile for the sediment, the suspended load transport can be written (an exponential profile for the concentration converges to a physical value when $z \rightarrow 0$, allowing for $\left.z_{R}=0\right)$ :

$$
\begin{gathered}
q_{s s}=U_{c} \int_{0}^{h} c_{R} \exp \left(-\frac{W_{s}}{\varepsilon} Z\right) d z \\
=U_{c} c_{R} \frac{\varepsilon}{W_{s}}\left[1-\exp \left(-\frac{W_{s} h}{\varepsilon}\right)\right]
\end{gathered}
$$


In solving the integral, the ratio $W_{s} h / \varepsilon$ may be usually be taken as large, so that the exponential term $\exp \left(-W_{s} h / \varepsilon\right) \approx 0$. This assumption that integrating to infinity or $h$ produces approximately the same result may not be valid in strong mixing if wave breaking is included. Integrating to the water surface only is straightforward, but results in an extra term involving an exponential function (Equation 210).

Equation 210 together with the expressions for the sediment diffusivity (Equation 164) and the reference concentration (Equation 185 and 186) allow for prediction of the suspended load in for a current, waves and current combined, and for breaking waves.

\section{Experimental data}

To investigate the suspended sediment transport in steady conditions and for the current and wave interaction, data sets covering a wide range in parameter values was compiled and analyzed. For a steady current, the same data set as for the study of the sediment diffusivity and reference concentration was used (Table 15). For the wave and current interaction, only data sets where the mean current (and preferably a velocity profile) was estimated could be used to calculate the total suspended load. Table 33 summarizes these data sets (see also Table 19).

\section{Validation of hypothesis}

To validate the two main hypotheses of this formula, i.e., an exponential profile of the sediment concentration and a constant velocity over depth, a comparison was performed between the measurements of the sediment suspended load and Equation 210 using the fitted values to the observed data for $c_{R}$ and $\varepsilon$.

Concerning the hypothesis of a constant value on the mean current over the water depth, a remark may first be made that it does not greatly influence the final results. Thus, knowing the concentration profile, use of the mean current $U_{c}$ causes an increase in the total suspended load of less than 5 percent compared to a vertical logarithmic velocity profile in the case of a steady current. For a cross-shore current (undertow), the velocity profile may be considerably more complex. Applying the model by Rattanapitikon and Shibayama (2000) for the undertow profile, a mean current $U_{c}$ induces a maximum overestimation of the suspended load of about 20 percent compared to the theoretical profile. To conclude, it 
appears that the error in the suspended load due to the chosen velocity profile is not significant compared to possible errors in the prediction of the mean current and suspended sediment concentration profiles.

Cases with steady current only. Figure 78 plots the observed suspended sediment load against the calculated suspended sediment load using Equation 210 with the empirical values of $c_{R}$ and $\varepsilon$ for the data with current only. The percentage of values obtained with an error less than a factor of 2 or 5 (designed as $P x 2$ or $P x 5$ ), as well as the mean value and the standard deviation of the function $f\left(q_{s s}\right)=\log \left(q_{s s, \text { pred }} / q_{s s, \text { meas }}\right)$, are presented in Table 34. The results indicates that the assumptions of an exponential concentration profile and a constant velocity over the depth are sufficient to estimate the suspended load for a steady current with a logarithmic vertical velocity profile.

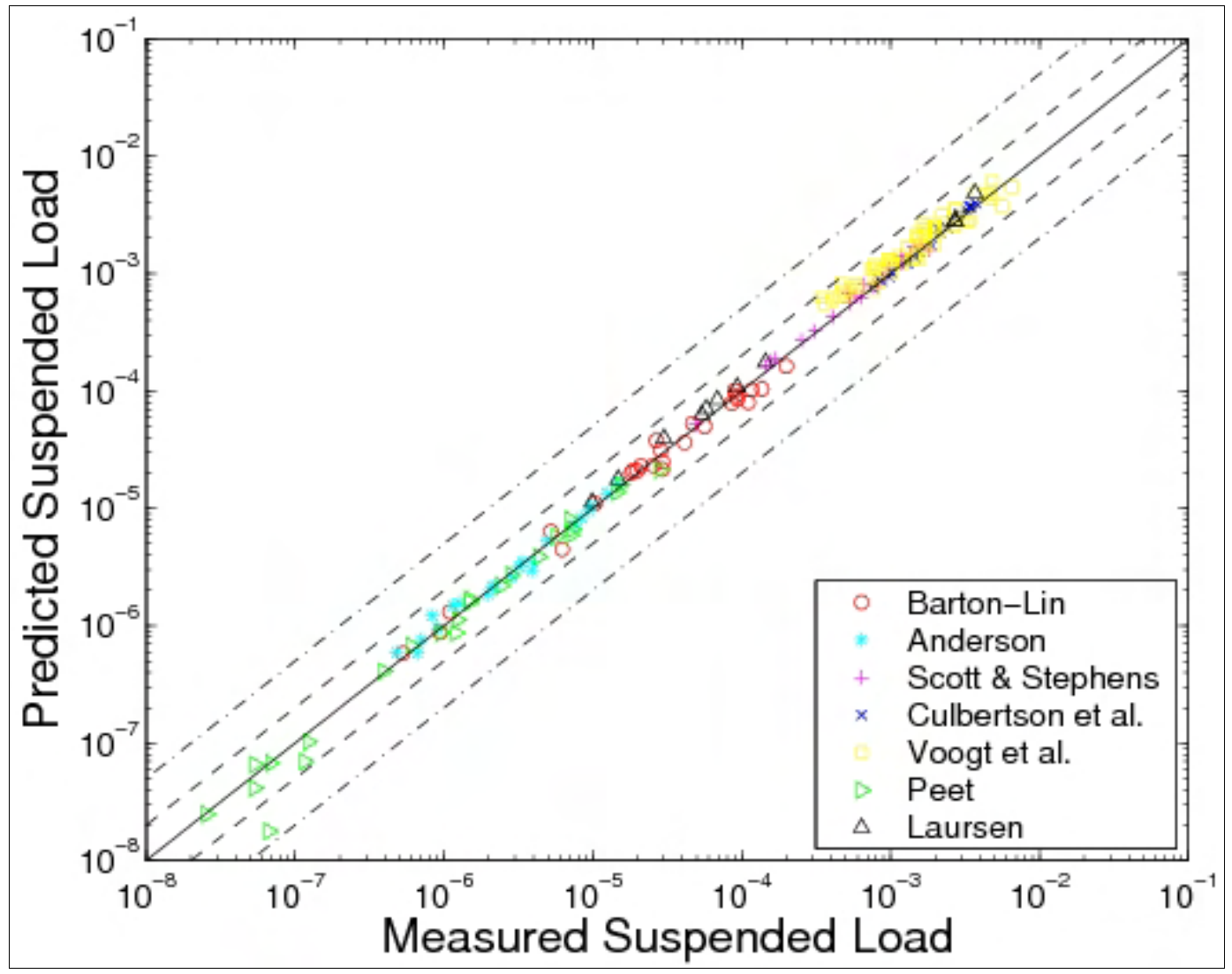

Figure 78. Comparison between observed and calculated suspended sediment load for steady current using Equation 210 with empirical values on $c_{R}$ and $\varepsilon$. 
Table 34. Prediction of suspended load transport for steady current.

\begin{tabular}{|l|l|l|l|l|}
\hline Author(s) & $P \times 2(\%)$ & $P \times 5(\%)$ & Mean $\left(f\left(c_{R}\right)\right)$ & Std $\left(f\left(c_{R}\right)\right)$ \\
\hline Equation 210 with observed $c_{R}$ and $\varepsilon$ & 99 & 100 & 0.03 & 0.09 \\
\hline Equations 210, 164, and 185 & 37 & 79 & -0.10 & 0.57 \\
\hline Bijker (1968) & 24 & 45 & 0.60 & 1.04 \\
\hline Engelund and Hansen (1972) & 31 & 55 & 0.65 & 0.85 \\
\hline Bailard (1981) & 33 & 72 & 0.32 & 0.69 \\
\hline Van Rijn (1984b, 1993) & 30 & 69 & -0.27 & 0.98 \\
\hline
\end{tabular}

Cases with wave and current interaction. Figure 79 plots measured (estimated) suspended sediment load against the calculated suspended sediment load calculated with Equation 210 with the measured values of $c_{R}$ and $\varepsilon$ for the data with wave and current interaction. The results are not as good as for the steady current data. Only 44 and 83 percent of the data are predicted within error factors of 2 and 5, respectively, (Table 35).

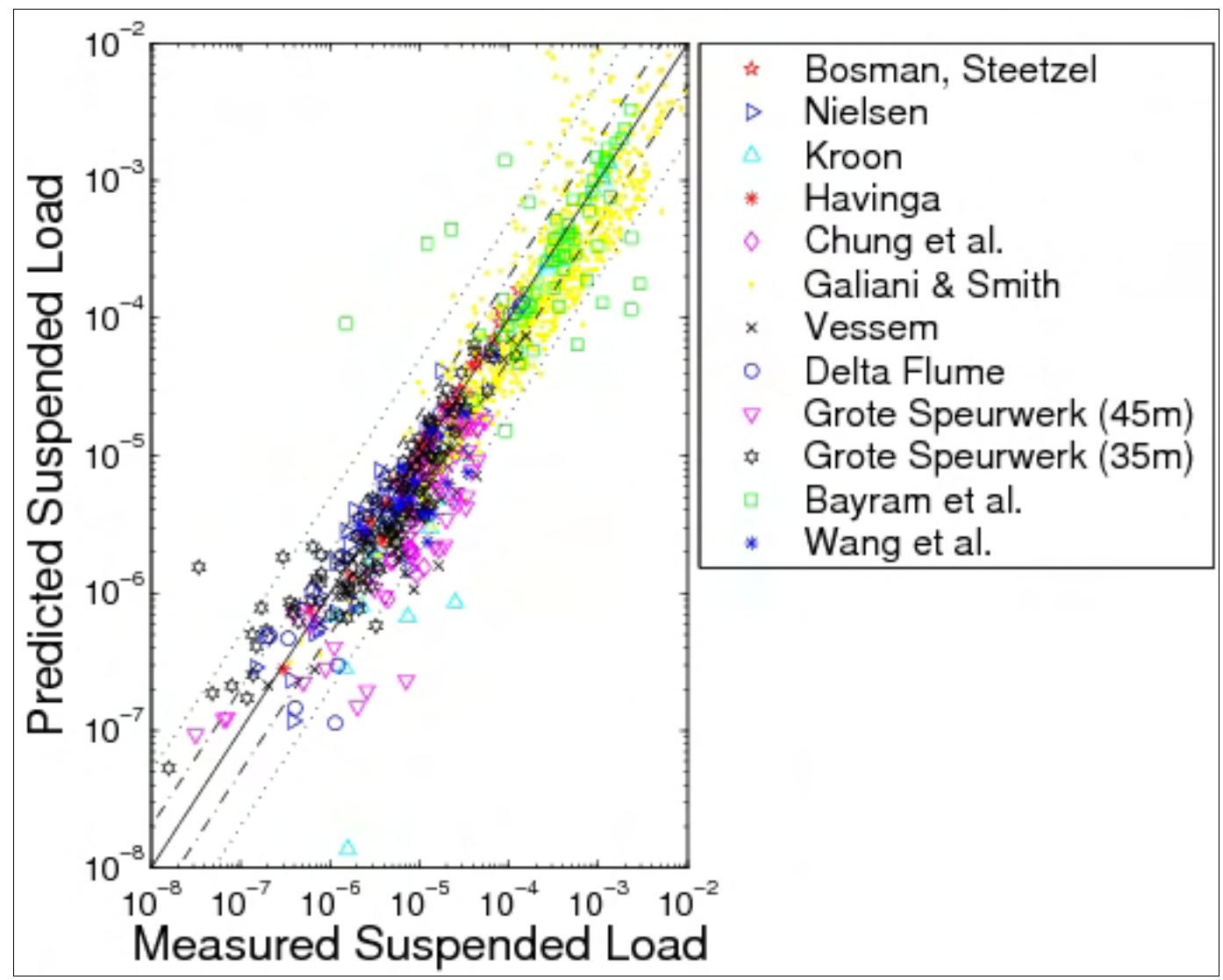

Figure 79. Comparison between observed and calculated suspended sediment load for wavecurrent interaction using Equation $\mathbf{2 1 0}$ with experimental values on $c_{R}$ and $\varepsilon$. 
Table 35. Prediction of suspended load transport for interaction between current and waves.

\begin{tabular}{|l|l|l|l|l|l|}
\hline Author(s) & $P \times 2(\%)$ & $P \times 5(\%)$ & Mean $\left(f\left(c_{R}\right)\right)$ & Std $\left(f\left(c_{R}\right)\right)$ \\
\hline \multicolumn{5}{|c|}{ All Data with Nonbreaking Waves Except Gailani and Smith Data Set } \\
\hline Equation 210 with observed $c_{R}$ and $\varepsilon$ & 67 & 91 & -0.16 & 0.38 \\
\hline Equations 210, 164, and 185 & 33 & 65 & 0.17 & 0.74 \\
\hline Bijker (1968) & 23 & 52 & 0.50 & 0.73 \\
\hline Bailard (1981) & 30 & 65 & 0.43 & 0.62 \\
\hline Van Rijn (1989) & 28 & 59 & -0.36 & 0.80 \\
\hline \multicolumn{5}{|c|}{ Gailani and Smith Data Set } \\
\hline Equation 210 with observed $c_{R}$ and $\varepsilon$ & 30 & 77 & -0.49 & 0.40 \\
\hline Equations 210, 164, and 185 & 24 & 52 & 0.25 & 0.91 \\
\hline Bijker (1968) & 25 & 54 & -0.35 & 0.84 \\
\hline Bailard (1981) & 26 & 51 & -0.48 & 0.90 \\
\hline Van Rijn (1989) & 15 & 36 & -0.77 & 1.26 \\
\hline \multicolumn{5}{|c|}{ All Data with Breaking Waves } & \multicolumn{2}{l|}{} \\
\hline Equation 210 with observed $c_{R}$ and $\varepsilon$ & 72 & 94 & -0.17 & 0.32 \\
\hline Equations 210, 164, and 185 & 32 & 70 & -0.09 & 0.69 \\
\hline Bijker (1968) & 27 & 68 & 0.20 & 0.69 \\
\hline Bailard (1981) & 50 & 84 & 0.28 & 0.50 \\
\hline Van Rijn (1989) & 18 & 49 & -0.12 & 0.81 \\
\hline
\end{tabular}

The suspended load is often overestimated. However, most of the cases where the suspended load is overestimated correspond to the large data set provided by Gailani and Smith (2000), where no measurements of the velocity were available close to the bottom. Thus, large uncertainty is encountered in estimating the suspended load (fitting the experimental data and using the coefficients for $c_{R}$ and $\varepsilon$ ). Also, for many cases (the GroteSpeurwerk (45 m) data set (Van Rijn et al. 2001), for example), measurements of the bed features were not reported. The estimation of the suspended load is strongly dependent on the Nikuradse roughness, or the bed-form height, as $z_{R}$ is considered to be equal to half of the bed-form height if such features are present (Van Rijn 1984c).

For a predominant longshore current, the velocity profile may be assumed to be equivalent to that of a steady current (logarithmic profile). On the other hand, on a cross-shore section, the velocity profile cannot be represented by a logarithmic velocity profile. It should be noted that for the Kroon (1991) data set, there are 31 suspended load profiles for which 
velocity profiles are provided alongshore and across shore. The suspended load is better predicted for the longshore component, especially if the mean undertow is small. A representative constant value for the velocity profile may be the mean value of the undertow over the water depth under the trough of the wave. Figure 80 presents (a) some typical cross-shore velocity profiles, and (b) concentration profiles (1) inside the surf zone, and (2) close to the breaker line. The velocity profiles correspond to a flume experiment by Svendsen and Hansen (1988), whereas the sediment concentration profiles correspond to a field experiment (Lubiatowo Beach, Baltic Sea, Poland) by Antsyferov et al. (1983). Even if the vertical profile of the undertow (circles) is clearly different from the classical velocity profile (dashed line), it appears that the mean undertow (averaged over the water depth under the trough of the waves; solid line) yields an accurate representation of the velocity.

With reference to Figure 80, if the wave rollers are not established, the velocity profile is nearly constant over depth and the sediment diffusivity is small. This implies that the greatest concentration and the main suspended load occur close to the bottom, where a logarithmic shape well represents the velocity profile. Following the results obtained for the steady current, using the mean undertow velocity in Equation 210 should allow for an accurate estimate of the suspended load (giving a slight overestimation). From Antsyferov et al. (1983), because the exponential profile tends to underestimate the sediment concentration close to the bed, the error in the total suspended load over the water depth may even be smaller.

In the middle of the surf zone, where the rollers are established, the velocity profile is not constant over depth. However, because the available energy is large, the sediment diffusivity is also large, so the concentration, as well as the suspended load, is more homogeneous over the water depth. A constant value on the velocity produces an underestimation of the suspended load for $z / h<0.3$, but an overestimation of the suspended load for $0.3<z / h$. Thus, the error in the total suspended load over the water depth may not be significant. However, there should be a tendency to overestimate the suspended sediment load if the sediment diffusivity is not sufficiently large. 


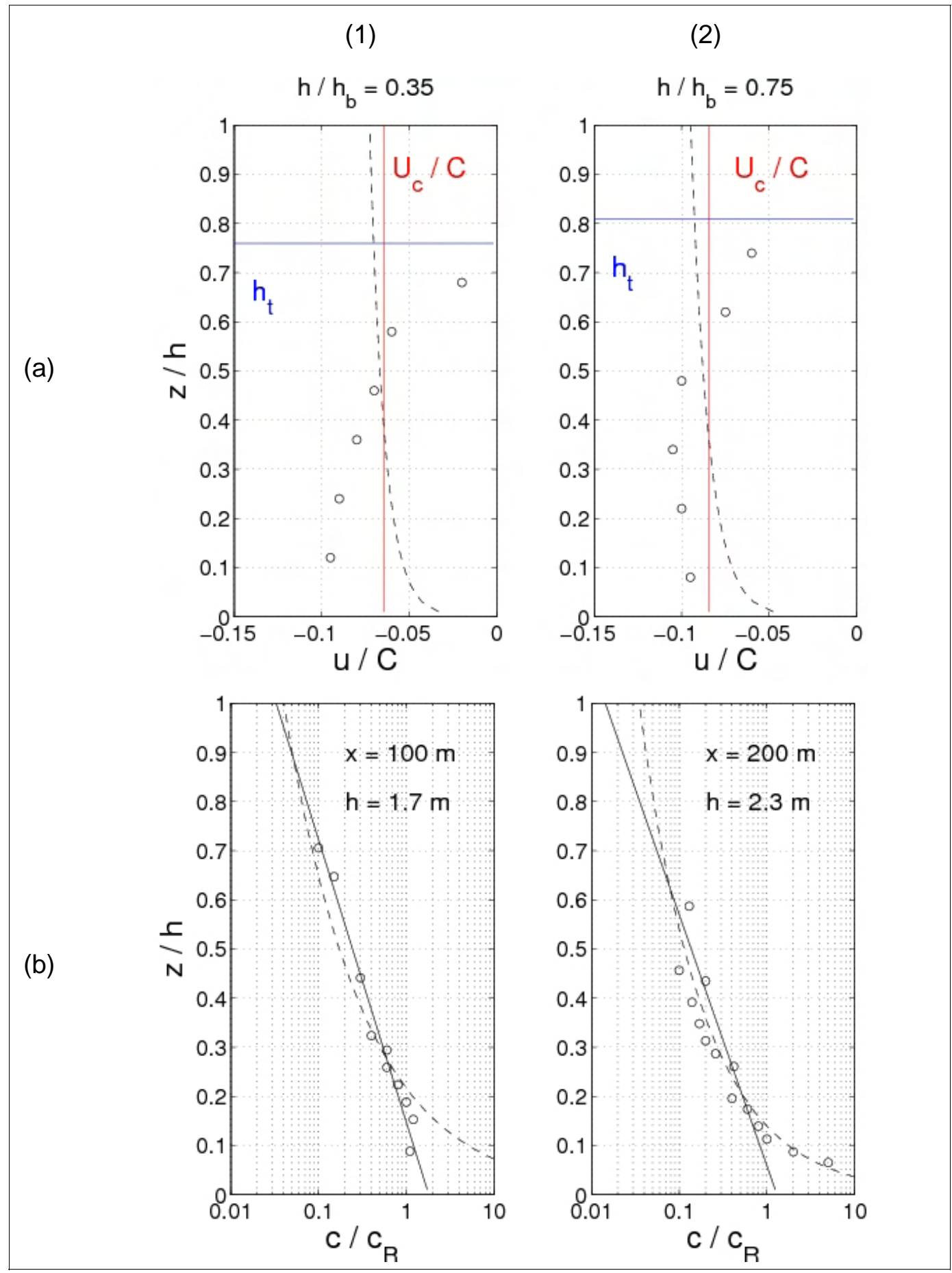

Figure 80. (a) Vertical velocity profile, and (b) sediment concentration profile, (1) inside surf zone, and (2) close to breaker line (after (a) Svendsen and Hansen 1988, and

(b) Antsyferov et al. 1983). Circles correspond to experimental data. For velocity profiles (a), theoretical logarithmic profile is included (dashed line). For suspended load profiles (b), solid line corresponds to an exponential profile, and the dashed line corresponds to power-law profile $\left(h_{t}\right.$ denotes the depth at wave trough level). 


\section{Comparison with experimental data in case of current only}

Measured and predicted suspended sediment load are compared in Table 34 and Figure 81. Overall, the proposed formula (Equation 210) shows correct behavior. The obtained results, however, appear to depend on the estimation of the reference concentration. Thus, using the equations proposed for $c_{R}$ for a steady current only (i.e., Equations 210 and 186) produce much better results than a constant value for $A_{c R}=510^{-4}$. An increase in the accuracy by nearly 10 percent and a decrease in the standard deviation by 6 percent can be observed. On the other hand, uncertainties in the prediction of the sediment diffusivity do not significantly affect the prediction of the suspended load transport.

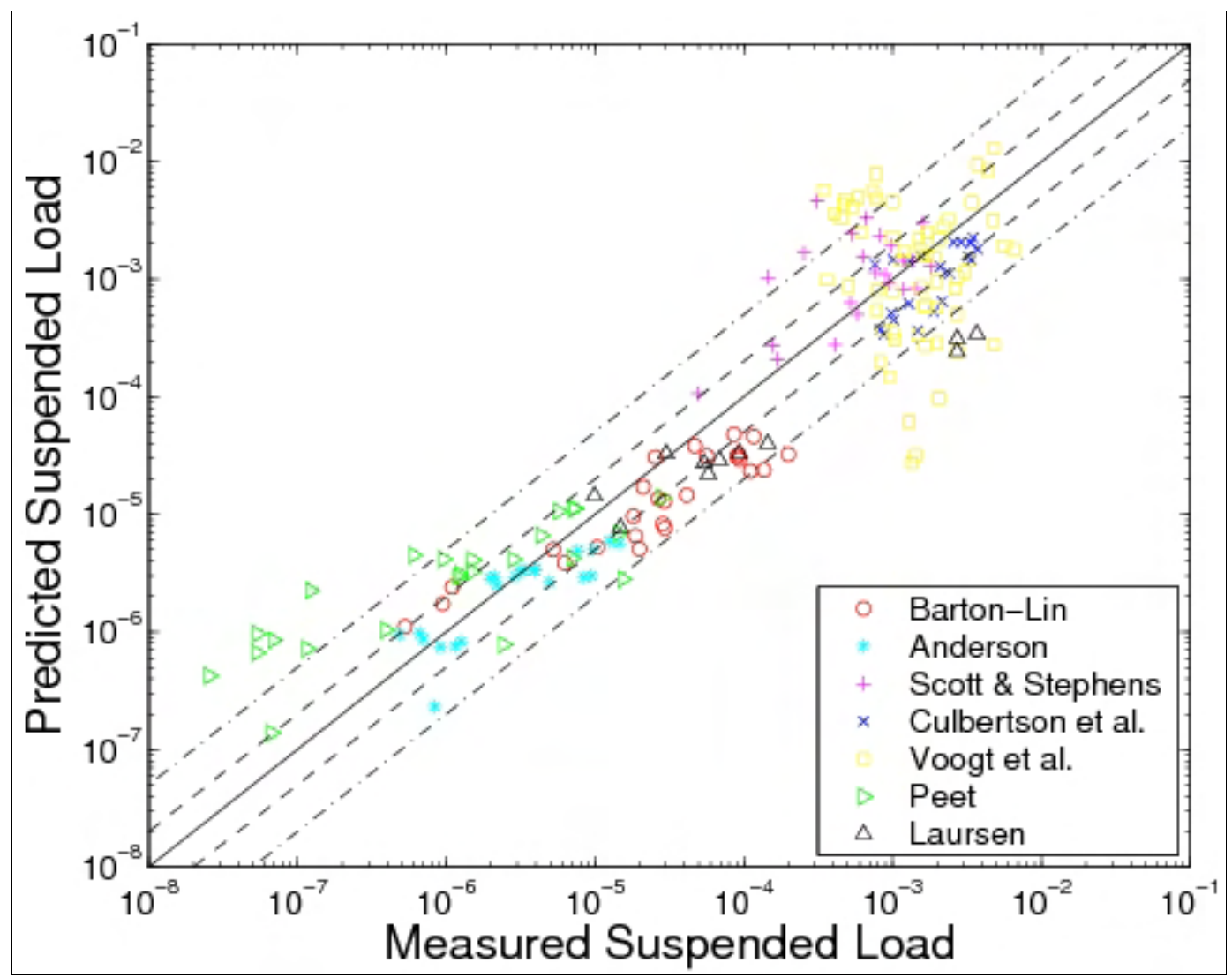

Figure 81. Comparison between observed suspended sediment load and calculated load using Equation 210 and predicted values for $C_{R}$ (Equation 185) and $\varepsilon$ (Equation 164) for current only.

Figure 81, exhibits similar behavior as for the reference concentration prediction: A general overestimation for the Anderson (1942), Scott and Stephens (1966), and Damgaard et al. (2003) data sets, and underestimation for the Barton and Lin (1955) and Laursen (1958) data. The 
reference concentration is sensitive to the dimensionless grain size (Equations 210 and 186). A comparison with other semi-empirical formulas found in the literature showed that the proposed relationship significantly improves the results only if Equation 185 is used for the reference concentration. Thus, $c_{R}$ appears to be the most significant parameter to determine in calculating suspended load.

\section{Comparison with experimental data for wave-current interaction}

Measured and predicted suspended sediment load for wave-current interaction are compared in Table 35 and Figure 82. It appears that the proposed formula (Equation 210) gives overall good results, although much more dispersion occurs compared to the steady current data.

Again, the results depend on estimation of the reference concentration, and thus, as previously shown, on estimation of the Nikuradse roughness and total shear stress. Indeed, using the equation proposed for $c_{R}$ for a steady current only (i.e., Equations 185 and 186) produces much better results than using a constant value for $A_{c R}=510^{-4}$. An increase in accuracy by nearly 10 percent, and a decrease in the standard deviation by 6 percent is obtained. Furthermore, plunging breaking waves may induce larger values on the reference concentration, as discussed. On the other hand, uncertainty from the prediction of the sediment diffusivity (in general, an overestimation) does not significantly affect the prediction of the suspended load transport. Thus, if an overestimation or underestimation is observed for the prediction of the reference concentration (Vessem data set (Van Rijn et al. 2001) and Kroon (1991) data set, respectively), the same observation can be made for the resulting suspended load. The percentage of values obtained within an error of a factor of 2 and 5 , as well as the mean value and the standard deviation of the ratio $f\left(q_{s}\right)=\log \left(q_{s, p r e d} /\right.$ $\left.q_{s, \text { meas }}\right)$, are presented in Table 35 for nonbreaking and breaking waves.

For wave-current interaction without breaking waves, the present work together with the Bailard (1981) formula yield the best results, even if marked scatter exists for the former. The Bailard (1981) formula, which does not include bed shear stress but velocities only, is less affected by the uncertainties in the Nikuradse roughness compared to the other formulas; thus, it yields smaller scatter. For the Van Rijn (1993) formula, the large number of parameters and its relative complexity may explain the observed scatter as it is more sensitive to any given parameter. 
(a)
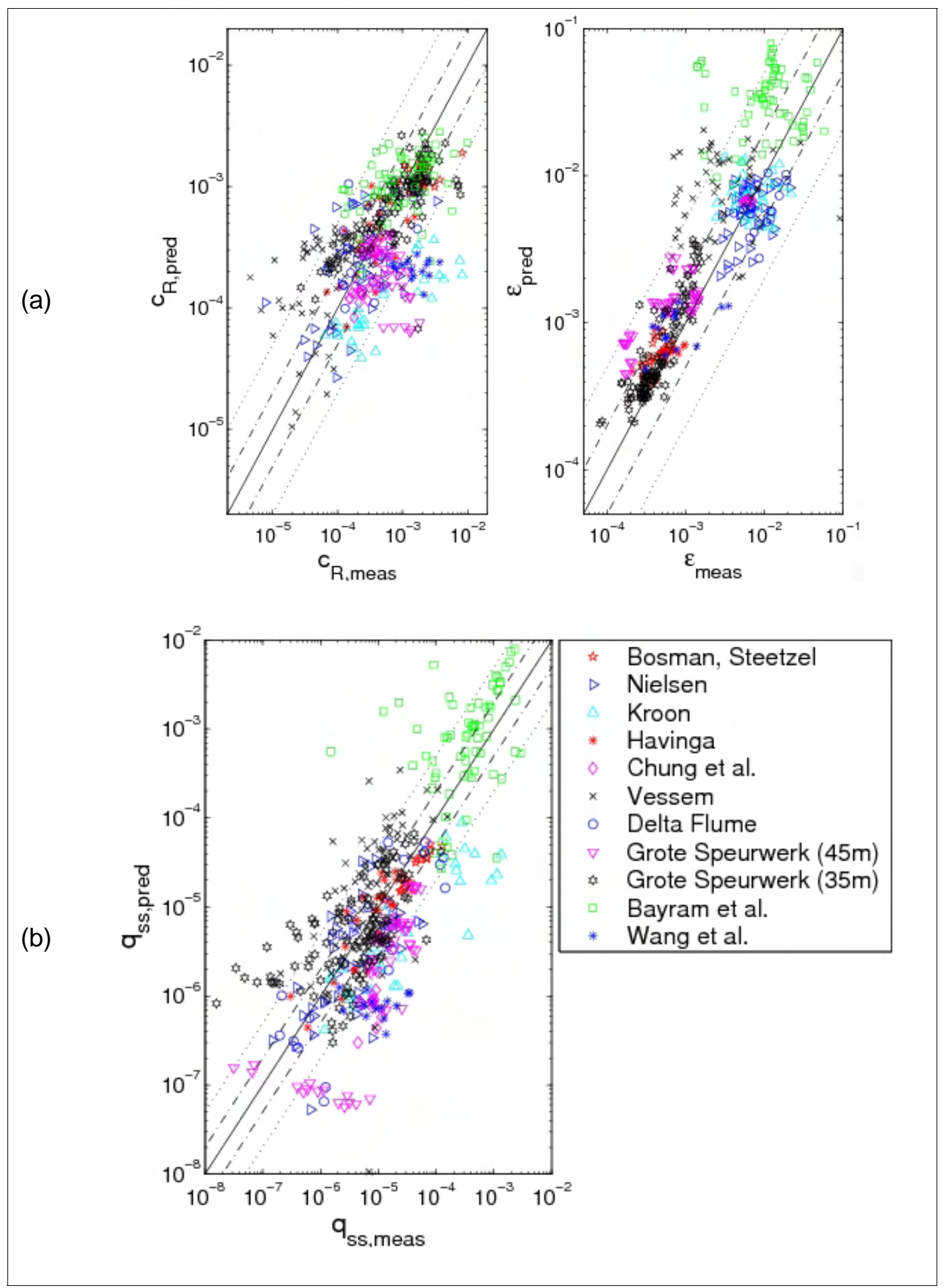

Figure 82. Comparison between measured and calculated values of wave and current interaction for (a) reference concentration $c_{R}$ (Equation 192) and sediment diffusivity $\varepsilon$ (Equation 164), and (b) for resulting suspended sediment load using Equation 210. 
The result obtained using the Van Rijn (1993) formula is poorer if waves are breaking. It appears that the Bijker (1968) formula and especially the Bailard (1981) formula present the best results among the studied formulas for this situation. These formulas were calibrated for estimation of the suspended load in the surf zone, thus yielding good predictions under breaking waves. The Bailard (1981) formula also produces less dispersion. This formula is not sensitive to the shear stress (only an average friction coefficient is introduced) and is simple enough to reduce the dispersion of the results. No improvement of the results could be obtained because the formula is basically only a function of the current and wave velocities at the bottom. For Equation 210, significant improvements in the results could be obtained with better predictions of the total shear stress. The formula tends to give an underestimate if waves are breaking (see the Kroon (1991) and Wang et al. (2002) data set in Figure 82b).

If Equation 196 is used (includes breaking wave effects), the results are improved for the Kroon 1991) and Wang et al. (2002) data sets, but not for the Grote Speurwerk ( $35 \mathrm{~m}$ ) and Vessem data sets. It should be noted that the overestimation observed for the Vessem and Bayram et al. (2001) data is mainly due to an overestimation of the sediment diffusivity. These two data sets correspond to greater water depth $(h>2 \mathrm{~m})$.

\section{Suspended sediment transport for rippled beds}

The typical bottom seaward of the surf zone has a rippled bed. Across shore, where the current is often weak and asymmetric waves prevail, and estimating the suspended load is difficult due to the interaction between bed forms and waves. These bed forms strongly affect the sediment transport by enhancing the suspended load, but also by creating a phase lag in the sediment suspension and sometimes modifying the direction of the sediment transport. In this situation, the wave-induced suspended load may dominate over the current-induced suspended load. Most semiempirical formulas are not valid because the wave-related suspended load $q_{s s, w}=<q_{s s}(t)>-q_{s s, c}$ (where $<>$ means a time-averaged value) is neglected. 


\section{Effects of ripples on suspended load}

Oscillatory wave motion over a rippled bed causes strong vortex motion that generates suspended sediment clouds, which move upward, forward, and backward in the water column (Figure 83). Spatial and temporal sediment concentration variability is, thus, relatively large. Furthermore, for asymmetric waves, the phase difference between the wave motion and the sediment concentration may result in offshore-directed net transport rates, because the suspended sediment clouds created during the first half period is transported the following half period in the opposite direction.

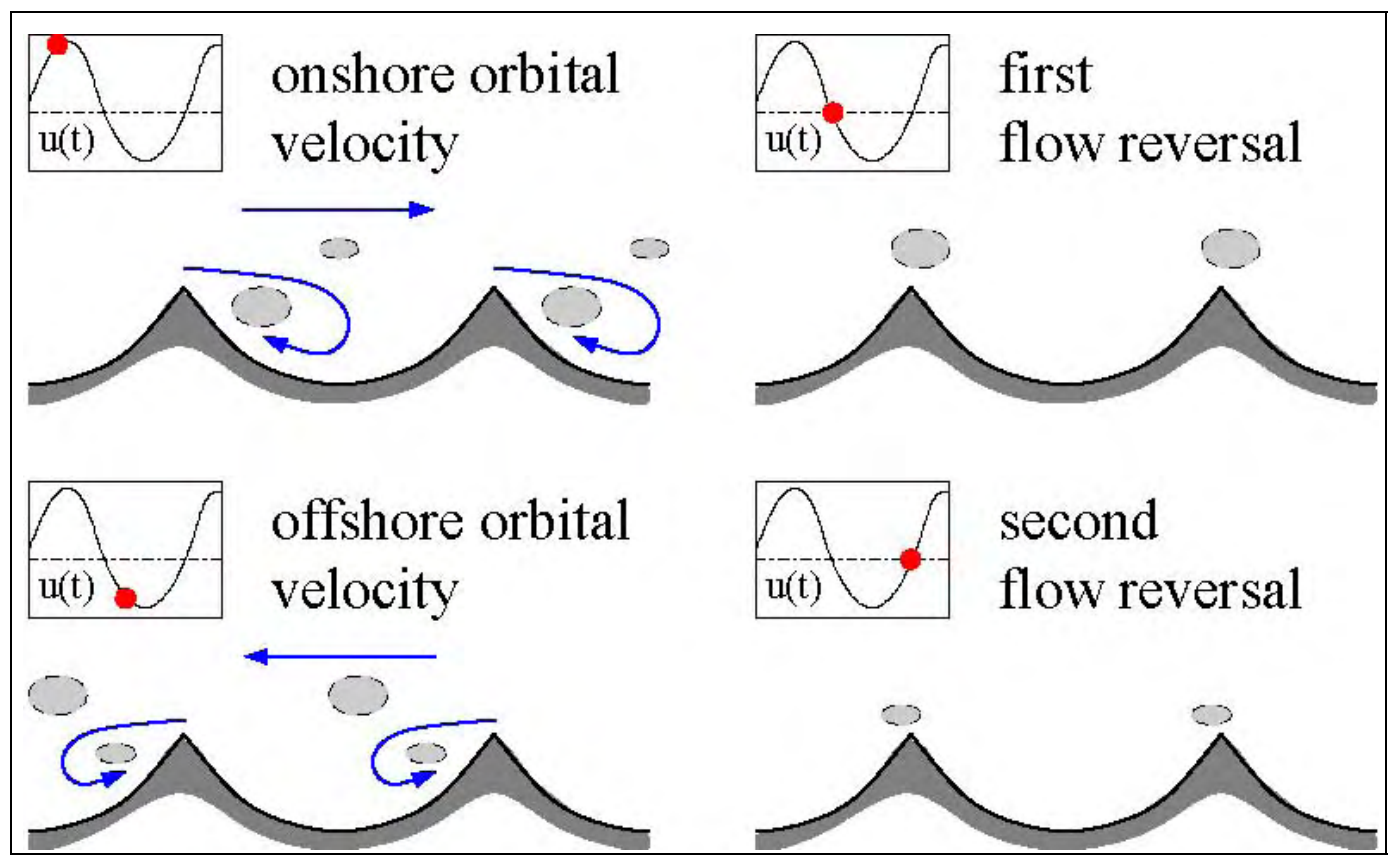

Figure 83. Schematic of transport processes in asymmetric wave motion over rippled bed.

These effects correspond to the wave-induced suspended sediment transport, as previously discussed (Equation 114):

$$
q_{s s, w}=<\int_{z_{a}}^{h(t)} \tilde{u}(z, t) \tilde{c}(z, t) d z>
$$

where $\tilde{c}(z, t)$ and $\tilde{u}(z, t)$ are the oscillatory components of the sediment concentration and velocity, respectively. This model is, however, difficult to apply directly because it is time-consuming and complicated to validate.

Some simplifications are necessary to estimate the wave-induced suspended load including the phase lag in a current-related formula, i.e., 
by assuming $<q_{s s, w}(t)>=X_{p l, s} q_{s s, c}$ where $X_{p l, s}$ is an empirical coefficient. The reduction in the sediment transport due to the phase lag may be defined as follows: $r_{p l, s}=q_{s s, n e t} / q_{s s, \phi_{p l}=0}=\left(q_{s s, c}+q_{s s, w}\right) / q_{s s, c}=1+X_{p l, s}$, where $q_{s s, n e t}=q_{s s, c}+q_{s s, w}$ is the net (total) suspended sediment transport, and $q_{s s, \phi_{p l}=0}=q_{s s, c}$ is the net suspended sediment transport if no phase lag occurs.

\section{Simple conceptual model for phase-lag effects on suspended load}

A simple conceptual model was introduced by Dohmen-J anssen (1999) to quantify the phase lag in sheet flow transport. This conceptual model may be extended to suspended load transport assuming that the instantaneous suspended load transport averaged over the water depth to be proportional to the instantaneous sediment concentration multiplied by the instantaneous horizontal velocity, both quantities being averaged over the water depth. Then, assuming that the instantaneous sediment concentration is a function of the instantaneous velocity to the power 2 , but with a possible phase lag, the effect of this phase lag on the sediment transport may be estimated. Using a velocity variation following second-order Stokes wave theory, the instantaneous velocity may be written $u(t)=U_{c}+$ $U_{w}\left[\cos (\omega t)+r_{w} \cos (2 \omega t)\right]$, and the instantaneous sediment concentration $c(t)=c_{0}\left[u\left(t+\phi_{p l}\right)\right]^{2}$, where $\phi_{p l}$ is the phase lag between the sediment suspension and the fluid velocity. The reduction in the sediment transport due to the phase lag compared to a case without phase lag may be obtained as follows:

$$
\begin{aligned}
r_{p l, s}= & \frac{q_{s s, n e t}}{q_{s s, \phi_{p l}=0}} \\
& =\frac{r_{u}^{3}+r_{u}\left[1 / 2+X_{p}+r_{w}^{2}\left(2 X_{p}^{2}-1 / 2\right)\right]+r_{w} / 2\left(X_{p}^{2}+X_{p}-1 / 2\right)}{r_{u}^{3}+3 / 2 r_{u}\left(1+r_{w}^{2}\right)+3 / 4 r_{w}}
\end{aligned}
$$

where $r_{u}=U_{c} / U_{w}, U_{c}$ is the mean current averaged over the depth, and $X_{p}$ $=\cos \phi_{p l}$, and $q_{s s, \phi p l=0}$ is the net sediment transport if no phase lag occurs.

It can be observed (Figure 84) that Equation 213 yields a larger reduction of the sediment transport with increasing $\phi_{p l}$. The direction of the sediment transport may also be modified if $r_{u}<0.5$ and $\phi_{p l}$ is close to $\pi / 2$. 


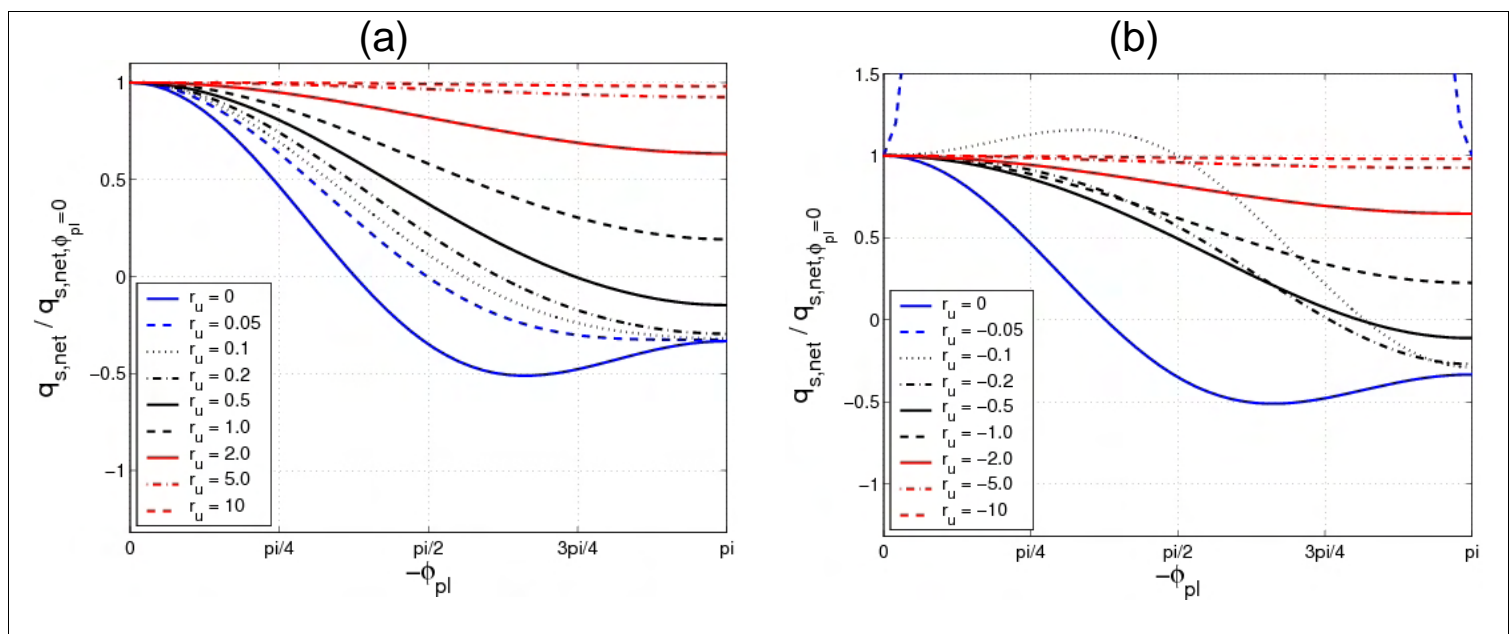

Figure 84. Phase-lag effects on sediment transport for second-order Stokes wave with (a) positive or (b) negative current introducing phase lag $\phi_{p l}$ for concentration at bottom and with asymmetry of $r_{w}=0.1$.

The net suspended sediment transport may also be simplified using the time-averaged sediment concentration and characteristic velocities for the onshore and offshore sediment transport. Assuming that no phase lag occurs, this yields:

$$
q_{s s, \phi_{p l}=0}=\frac{V_{c}+V_{t}}{U_{w}} \frac{1}{T_{w}} \int_{0}^{T_{w}} c(t) d t=\frac{V_{c}+V_{t}}{U_{w}}\left[r_{u}^{2}+\frac{1}{2}\left(1+r_{w}^{2}\right)\right]
$$

where $V_{c}$ and $V_{t}$ are characteristic velocities during the onshore and offshore motion, respectively. If a phase lag between the instantaneous concentration and velocity occurs, the characteristic onshore velocity is small compared to the mean concentration, whereas the characteristic offshore velocity is large. Thus, Equation 214 may be modified introducing an efficiency coefficient $\alpha_{p l, s}$ :

$$
q_{s s, n e t}=\frac{V_{c}\left(1-\alpha_{p l, s}\right)+V_{t}\left(1+\alpha_{p l, s}\right)}{U_{w}}\left[r_{u}^{2}+\frac{1}{2}\left(1+r_{w}^{2}\right)\right]
$$

with $0<a_{p l, s}<2$. Assuming as a first approximation, $V_{c} / U_{w}=r_{u}+$ $\sqrt{2} / 2\left(1+r_{w}\right)$ and $V_{t} / U_{w}=r_{u}-\sqrt{2} / 2\left(1-r_{w}\right)$, the coefficient $\alpha_{p l, s}$ is obtained from Equations 213, 214, and 215:

$$
\alpha_{p l, s}=\left(1-r_{p l, s}\right)\left(\sqrt{2} r_{u}+r_{w}\right)
$$




$$
=\frac{\left[r_{u}\left(1-X_{p}\right)+r_{u} r_{w}\left(1-2 X_{p}^{2}\right)+r_{w} / 2\left(2-X_{p}-X_{p}^{2}\right)\right]\left(\sqrt{2} r_{u}+r_{w}\right)}{r_{u}^{3}+3 / 2 r_{u}\left(1+r_{w}^{2}\right)+3 / 4 r_{w}}
$$

Figure 85 shows the relationship between $\alpha_{p l, s}$ and $\phi_{p l}$ for varying values of $r_{u}$. If $\left|r_{u}\right|<0.5$, large phase lag is predicted $\left(\alpha_{p l, s}>1\right)$ if $-\phi_{p l}>\pi / 2$. It should be noted that Equation 217 diverges if $r_{u}=r_{w} / 2$, because for this specific case the model predicts a net sediment transport rate equal to zero $\left(V_{c}=V_{t}\right)$.

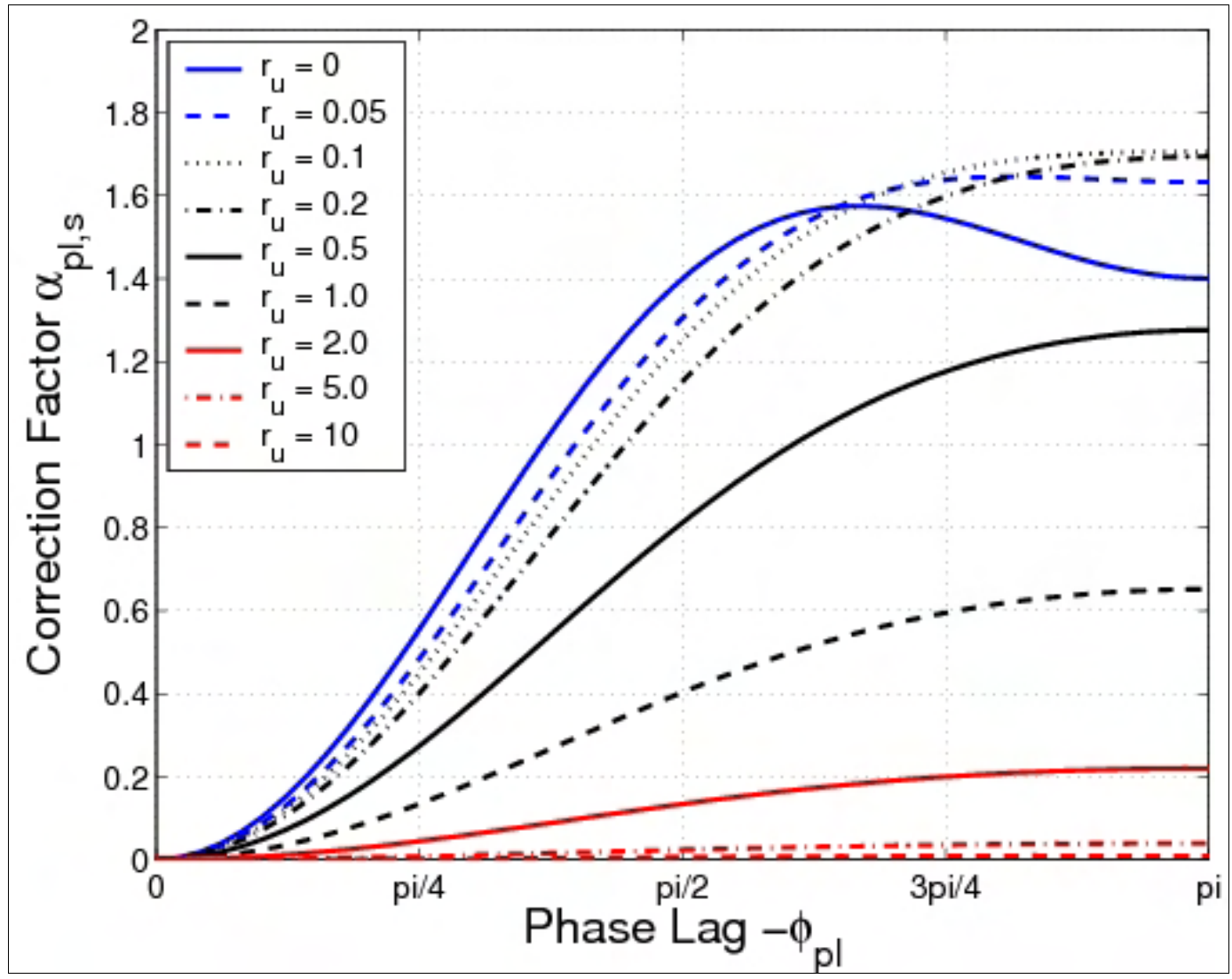

Figure 85. Coefficient $\alpha_{p l, s}$ as function of sediment phase lag $\phi_{p l}$ with varying values on $U_{c} / U_{w}$ and an asymmetry of $r_{w}=0.20$.

\section{Modification of formula for asymmetric waves}

For asymmetric waves, a net sediment transport may exist even if the mean steady current equals zero, because of the wave-related suspended load. To account for wave asymmetry, Equation 210 was modified by replacing $U_{c}$ with $U_{c, \text { net, }}$, where $U_{c, \text { net }}$ is defined as the "net" steady current, taking into account a possible phase lag of the sediment suspension: 


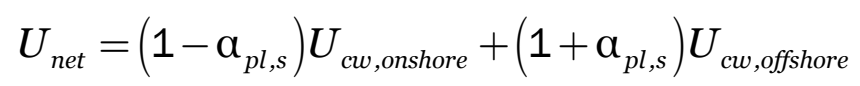

where $U_{c w, \text { onshore }}$ and $U_{c w, \text { offshore }}$ are the root-mean-square values of the total instantaneous velocity (wave and current: $u(t)=U_{c}+u_{w}(t)$ ) close to the bottom in the onshore direction $(u(t) \geq 0)$ or in the offshore direction $(u(t)<0)$, respectively (Figure 86). The coefficient $\alpha_{p l, s}$ describes the phase lag effect on the suspended load, decreasing $U_{c w, o n s h o r e ~}$ and increasing $U_{c w, o f f s h o r e ~}$ with increasing phase lag, assuming that the characteristic speed of the sediment concentration in the onshore (or offshore) direction is decreased (or increased). It should be noted that in case of a steady current without waves (or with sinusoidal waves), $U_{c, \text { net }}=U_{c}$.

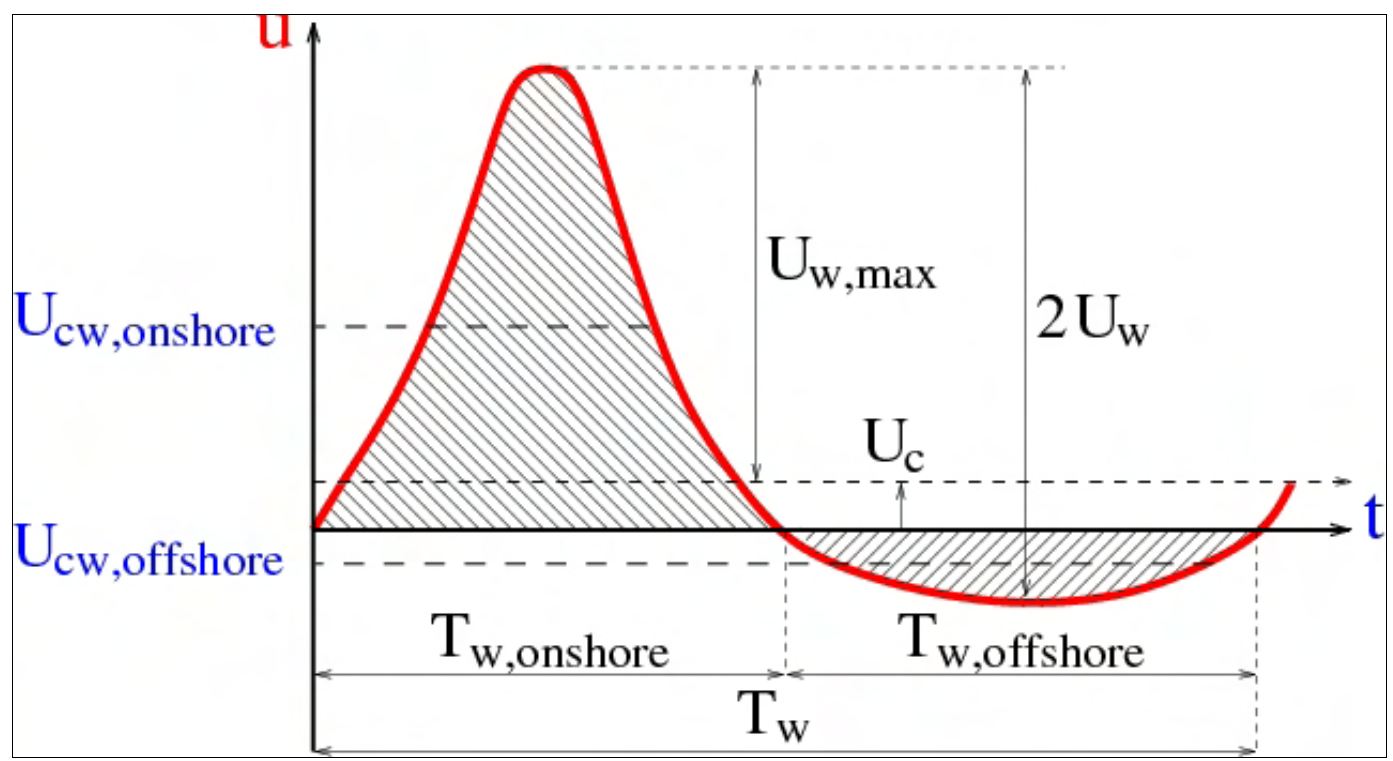

Figure 86. Notation for colinear wave and current interaction.

\section{Observations of phase-lag effects on suspended load over ripples}

To investigate phase-lag effects on suspended load transport over sandy ripples under combined waves and current, relevant data sets were compiled and analyzed. Only a few data sets were available for estimation of the net sediment transport rate including both current-related and wave-related suspended sediment transport. Table 36 summarizes the data sets employed, where the type of experiment, sediment characteristics, and wave properties are listed. 
Table 36. Summary of data on suspended sediment transport over ripples in full-cycle oscillatory flow.

\begin{tabular}{|l|l|l|l|l|l|l|l|l|l|}
\hline Author(s) & Exp. & Number & $\begin{array}{l}d_{50} \\
(\mathrm{~mm})\end{array}$ & $\begin{array}{l}U_{c} \\
(\mathrm{~cm} / \mathrm{sec})\end{array}$ & $\begin{array}{l}U_{w} \\
(\mathrm{~cm} / \mathrm{sec})\end{array}$ & $\begin{array}{l}r_{w} \\
(-) \times 100\end{array}$ & $\begin{array}{l}T_{w} \\
(\mathrm{sec})\end{array}$ & $\begin{array}{l}H_{r} \\
(\mathrm{~mm})\end{array}$ & $\begin{array}{l}L_{r} \\
(\mathrm{~mm})\end{array}$ \\
\hline Sato (1987) & OWT & 62 & 0.18 & 0 & $13-53$ & $4-66$ & $3.0-5.5$ & $7-27$ & $79-187$ \\
\hline $\begin{array}{l}\text { Ribberink } \\
\text { and Al Salem } \\
(1994)\end{array}$ & OWT & 6 & 0.21 & 0 & $28-47$ & $21-27$ & $5.0-9.1$ & $3-14$ & $84-110$ \\
\hline Clubb (2001) & OWT & 4 & 0.34 & 0 & $54-69$ & 26 & $5.0-10$ & $73-141$ & $510-770$ \\
\hline $\begin{array}{l}\text { Grasmeijer } \\
\text { (2002) }\end{array}$ & SWF & 20 & 0.10 & $-6--2$ & $27-52$ & $0-20$ & 2.3 & $5-13$ & $38-83$ \\
\hline $\begin{array}{l}\text { Van der Werf } \\
\text { and } \\
\text { Ribberink } \\
\text { (2004) }\end{array}$ & OWT & 10 & 0.35 & 0 & $42-85$ & 26 & $5.0-10$ & $25-139$ & $269-1,130$ \\
\hline
\end{tabular}

Using Equations 210 and 218 with $\alpha_{p l, s}=0$, which assumes that currentrelated suspended load dominates with a simple enhancement due to the presence of waves, large discrepancies are observed (Figure 87). Errors in the prediction of the net sediment transport are especially large for the Van der Werf and Ribberink (2004) data set where wave-related suspended load was prevailing.

As was shown by Nielsen (1992) and Ribberink and Al Salem (1994), Van der Werf and Ribberink (2004) observed that the vertical scale of the suspended sediment concentration profile is closely related to the ripple height, which scales reasonably well with the size of the vortices. They proposed to use the vortex suspension parameter to characterize the transport defined as follows:

$$
p_{W R}=\frac{H_{r}}{d_{50}}
$$

It is seen from Figure 88 that the direction of the net suspended transport rate depends on the parameter $p_{W R}$. The critical value of $p_{W R}$ up to which the net suspended load is directed offshore appears to be slightly larger than the one observed by Van der Werf and Ribberink (2004) $\left(p_{W R, c r}=100\right.$ instead of 70). However, large uncertainties exist in these estimates. 


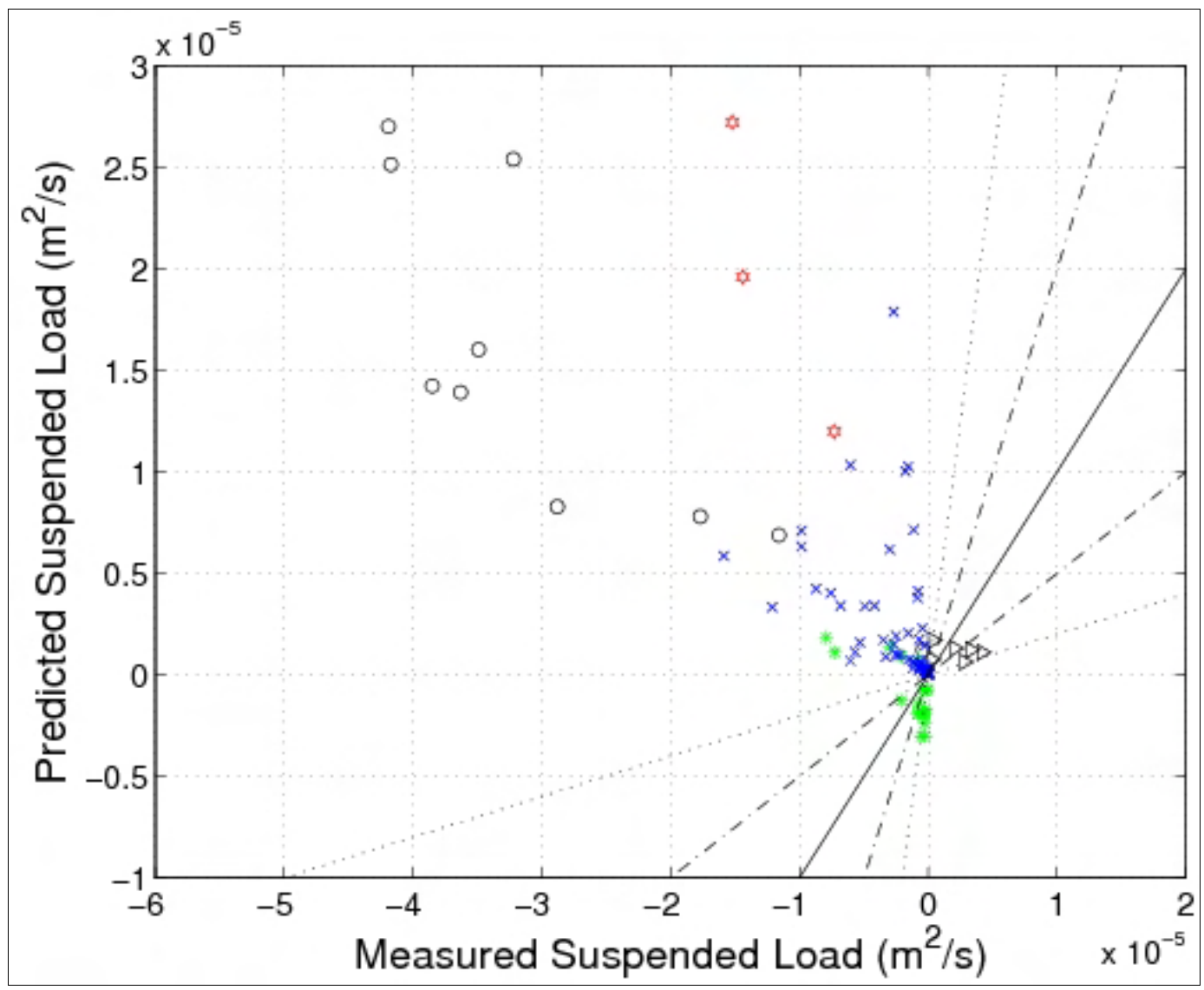

Figure 87. Comparison between measured and calculated net sediment transport rate using Equations 210 and 218 with $\alpha_{p l, s}=0$ (legend is same as in Figure 88).

\section{Empirical formulas for $\phi$ and $\alpha_{p l, s}$}

Table 37 lists predictions of the net suspended transport rate within a factor of $2(P \times 2)$ and $5(P x 5)$ of the measured values displayed ("factor of $\mathrm{x}^{\prime \prime}$ means between $x$ times and $1 / x$ times the measured net suspended transport rate). The table also presents the mean value of the ratio $f\left(q_{s s}\right)$ $=q_{s s, p r e d} / q_{s s, \text { meas. }}$ and its standard deviation. A perfect prediction leads to $f\left(q_{s s}\right)=1$. A negative value on $f\left(q_{s s}\right)$ means that the direction of the sediment transport is poorly predicted. 


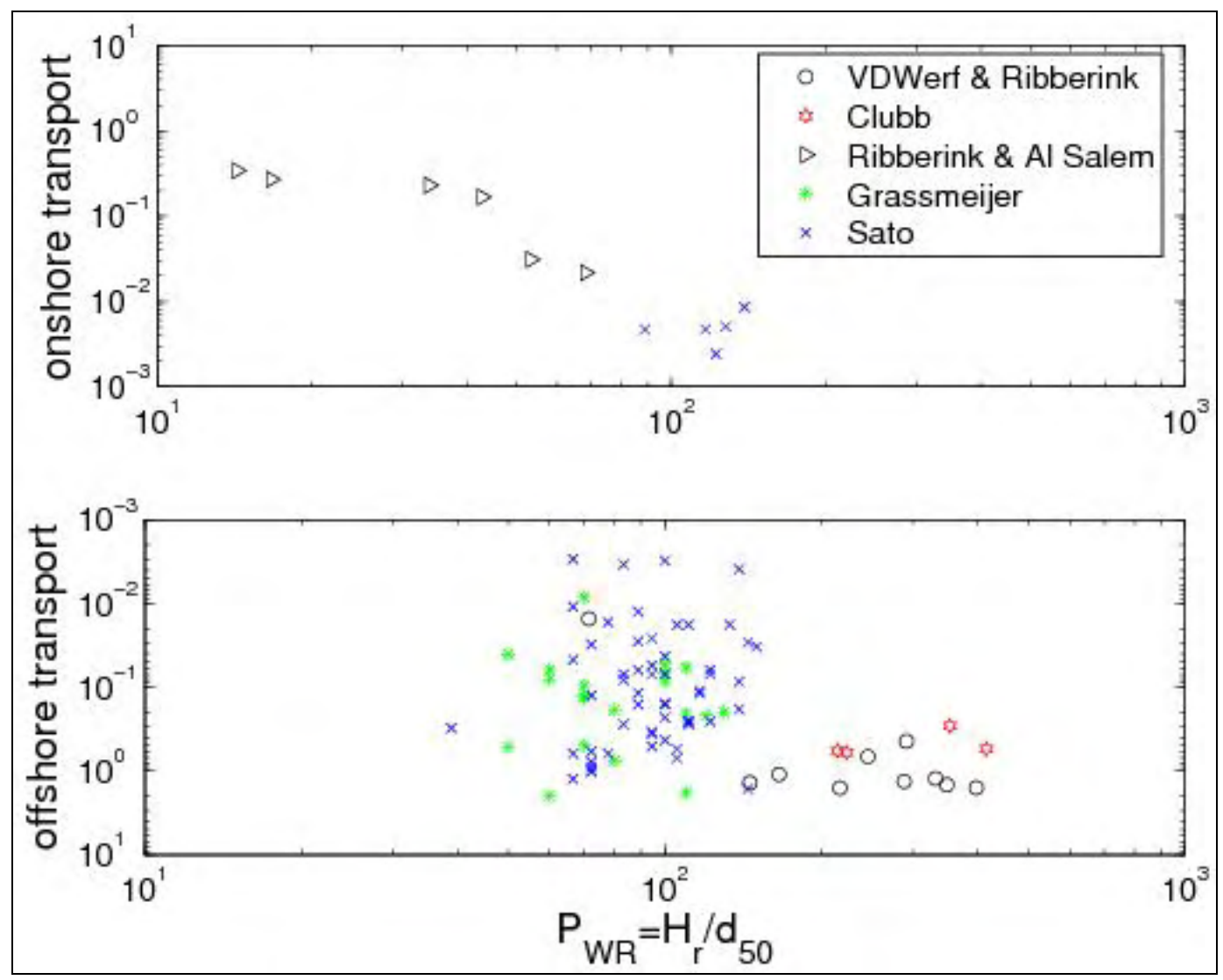

Figure 88. Dimensionless suspended sediment transport rate as function of phase-lag parameter $p_{W R}$.

Table 37. Prediction of suspended sediment transport rate within factor of 2 or 5 of measured values, together with mean value and standard deviation on $f\left(q_{s s}\right)$.

\begin{tabular}{|l|l|l|l|l|}
\hline Author(s) & $\begin{array}{l}\text { Px2 } \\
(\%)\end{array}$ & $\begin{array}{l}\text { Px5 } \\
(\%)\end{array}$ & Mean $\left(f\left(q_{s s}\right)\right)$ & Std $\left(f\left(q_{s s}\right)\right)$ \\
\hline Dibajnia and Watanabe (1992) & 15 & 25 & -3.5 & 2.38 \\
\hline Van der Werf and Ribberink (2004) & 20 & 34 & -2.8 & 2.50 \\
\hline Equations 210 and 218 with $\alpha_{p l, s}=0$ & 06 & 16 & -3.6 & 2.40 \\
\hline Equations 210 and 218 with $r_{p l, s}($ Equation 213) & 22 & 57 & -1.2 & 1.88 \\
\hline Equations 210, 218, 217, and 220 & 42 & 71 & -1.0 & 2.03 \\
\hline Equations 210, 218, and 221 & 46 & 69 & -0.7 & 1.93 \\
\hline
\end{tabular}

As a comparison, the predictions using the Dibajnia and Watanabe (1992) formula are also presented. This formula is a total load semi-empirical description that includes the effects of the phase lag for both ripple and sheet-flow regimes. It yields fairly good results; however, for situations with strong phase-lag effects (data from Van der Werf and Ribberink 
(2004) and some data from Sato (1987)), the direction of the net sediment transport is incorrectly estimated. Van der Werf and Ribberink (2004) proposed a modified version of the Dibajnia and Watanabe (1992) formula using the parameter $p_{W R}$ as a critical parameter for phase-lag inception and replacing the mobility parameter used by Dibajnia and Watanabe (1992) with the Shields parameter. It improves results, but still with a large scatter, especially for the data from Sato (1987). Equations 210 and 218 with $\alpha_{p l, s}=0$ yield poor results both because of the phase-lag effects, which are not taken into account, and because of excessive sensitivity for small grain size.

Calibration of the conceptual model with $r_{p l, s}$ (Equation 213) was particularly difficult. Indeed, an estimation of $r_{p l, s}$ was obtained by assuming that the sediment transport without phase lag $q_{s s, p r e d, \phi p l}=0$ is correctly predicted by Equations 210 and 218 with $\alpha_{p l, s}=0$, i.e., $r_{p l, s, \text { meas }}=q_{s s, \text { meas }} / q_{s, p r e d, \phi p l}=0$. This assumption leads to values on $r_{p l, s}$ equal either to 1 (no phase lag) or approximately less than - 1 , which is the minimum value from the conceptual model (Figure 84). Thus, the calibration of the parameter $\phi_{p l}$ leads to a simple equation that only includes the vortex suspension parameter $P_{W R}$ :

$$
\phi_{p l}=\pi \tanh \left[0.5\left(\frac{P_{W R}}{P_{W R, c r}}\right)^{2}\right]
$$

The introduction of the coefficient $r_{p l, s}$ significantly improves the results (Table 37). However, it underestimates the phase-lag effects for most of the data sets, because the minimum of $r_{p l, s}<-1$ (Figure 89(b)).

Using the same relationship for $\phi_{p l}$ with the conceptual model to estimate $\alpha_{p l, s}$ (Equation 217) yields good results for the prediction of the net sediment transport. More than 40 percent ( 70 percent) of the measurements are correctly predicted within a factor 2 (5) allowed. Most directions in the measurements are moreover correctly predicted. It appears (Figure 89(c)) that only the data set from Grasmeijer (2002), where large dispersion exists, is not well predicted. 


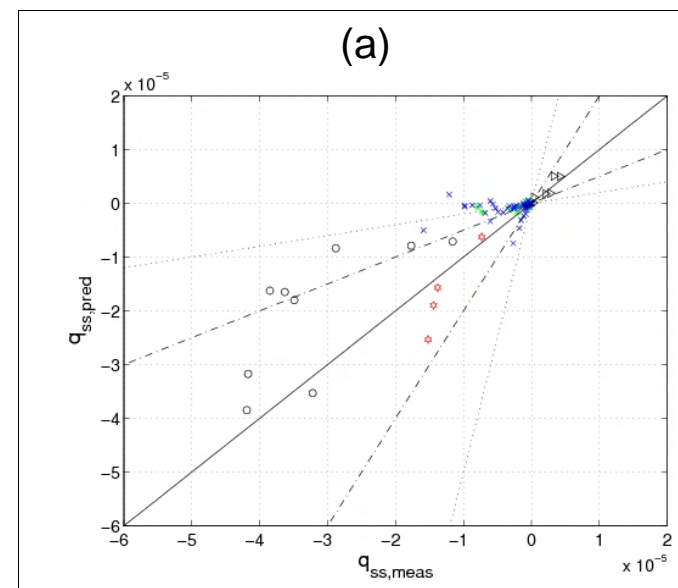

(c)

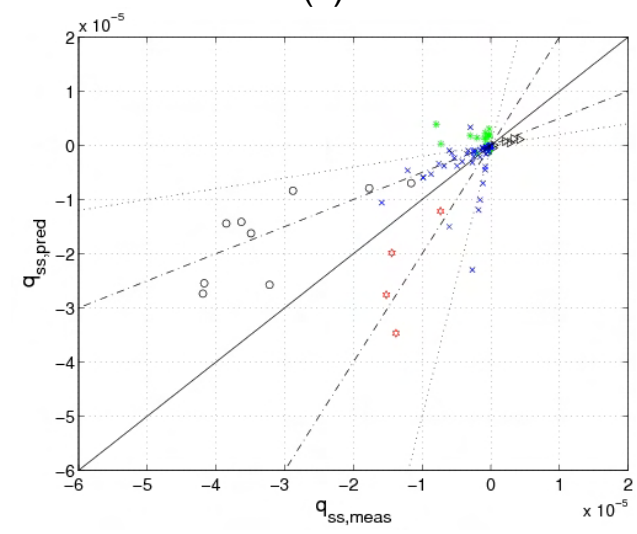

(b)

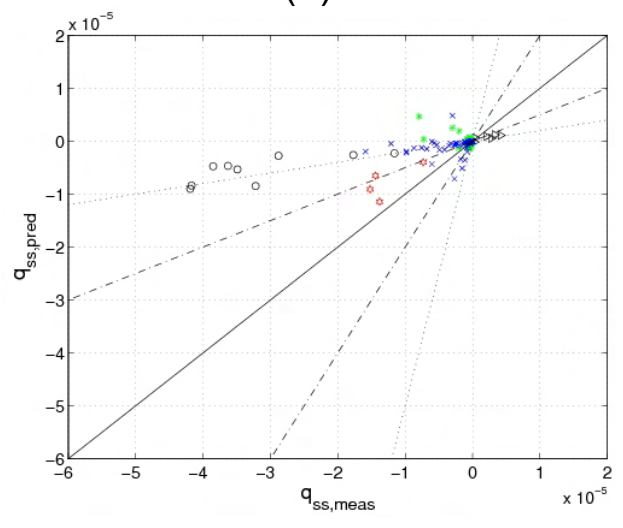

(d)

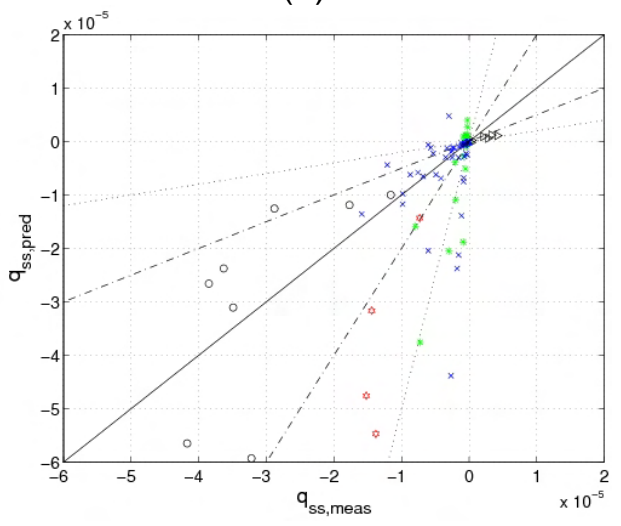

Figure 89. Comparison between measured and estimated net sediment transport rate using

(a) Van der Werf and Ribberink (2004) formula; (b) Equations 210 and 218 with $r_{p l, s}$ from Equation 213; (c) Equations 210 and 218 with $\alpha_{p l, s}$ from Equation 217; or (d) Equation 221.

To directly calibrate the coefficient $\alpha_{p l, s}$, an analysis was undertaken with respect to the wave and sediment characteristics. As for the Van der Werf and Ribberink (2004) formula, the vortex suspension parameter $p_{W R}$ was used to limit the effect of $\alpha_{p l, s}$ to cases where phase-lag effects may occur, i.e., when $p_{W R}>p_{W R, c r}$. To take into account the possible error on the critical vortex suspension parameter, an exponential function was introduced. Following the study on sheet flow phase lag, the coefficient $D_{p l}$ is calculated for each half-period ( $D_{p l, o n}$ and $\left.D_{p l, o f f}\right)$ :

$$
\alpha_{p l, s}=\left(D_{p l, o n}-D_{p l, o f f}\right) \exp \left[-0.25\left(\frac{p_{W R, c r}}{p_{W R}}\right)^{4}\right]
$$


where for each half-period, $D_{p l, i}$ (index $i$ refers to onshore or offshore) was found to be a function of the wave orbital velocity, period, and the settling velocity, through the following two dimensionless parameters:

$$
\begin{gathered}
v_{i}=\frac{U_{c w, i}}{W_{s}} \\
\omega_{i}=\frac{H_{r}}{T_{w, i} W_{s}}
\end{gathered}
$$

where the former is a suspension parameter and latter the ratio between the ripple height and the settling distance for each half-period of the wave. From the calibration, the resulting equation for $D_{p l, i}$ was:

$$
D_{p l, i}=A_{d} v_{i}^{0.5} \omega_{i}^{0.25}
$$

with $A_{d}=0.7$.

This latter equation yields the best overall results (Table 37 and Figure 89), although it tends to overestimate the phase-lag effects.

\section{Sensitivity analysis for different formulas}

To further understand the sensitivity of a phase lag on the different parameters, an analysis was undertaken for the wave orbital velocity, period, and asymmetry. For these tests, to be able to plot single curves, a constant value was used for the ripple height (mean value of the measurements). This assumption constitutes a limitation of the study. For all the graphics, "Camenen and Larson $\left(\alpha_{p l, s}-1-\right)$ " corresponds to Equations 210, 218, and 221; "Camenen and Larson ( $\left.\alpha_{p l, s}-2-\right)$ " corresponds to Equations 210, 218, 217, and 220; and "Camenen and Larson $\left(r_{p l, s}\right)$ " corresponds to Equations 210 and 218 with $r_{p l, s}$ (Equation 213).

Effect of wave orbital velocity. The phase lag is proportional to the wave orbital velocity. The greater $U_{w}$ is, the larger the amount of sediment put into suspension (the available energy is higher) and the larger the sheet flow layer thickness $\delta_{s}$. This implies a greater delay between the instantaneous concentration and shear stress and fluid velocity. Table 38 and Figure 90 show the obtained results for parameters from selected experiments. 
Table 38. Experiment conditions for studied cases on wave orbital velocity effects (mean value is used for ripple height $H_{r}$; for Grasmeijer (2002) experiment, mean value is also used for water depth $h$ and velocity $U_{c}$ ).

\begin{tabular}{|l|l|l|l|l|l|l|l|}
\hline Case & Author(s) & $\begin{array}{l}d_{50} \\
(\mathrm{~mm})\end{array}$ & $\begin{array}{l}h \\
(\mathrm{~m})\end{array}$ & $\begin{array}{l}U_{c} \\
(\mathrm{~m} / \mathrm{sec})\end{array}$ & $\begin{array}{l}T_{w} \\
(\mathrm{sec})\end{array}$ & $\begin{array}{l}V_{w} \\
(-)\end{array}$ & $\begin{array}{l}H_{r} \\
(\mathrm{~mm})\end{array}$ \\
\hline a & Van der Werf and Ribberink & 0.35 & 1.10 & 0 & 7.5 & 0.26 & 8.2 \\
\hline b & Grasmeijer & 0.10 & 0.46 & -0.03 & 2.3 & 0.05 & 8.7 \\
\hline c & Sato & 0.18 & 0.30 & 0 & 3.0 & 0.25 & 1.7 \\
\hline d & Sato & 0.18 & 0.48 & 0 & 3.7 & 0.20 & 1.7 \\
\hline
\end{tabular}

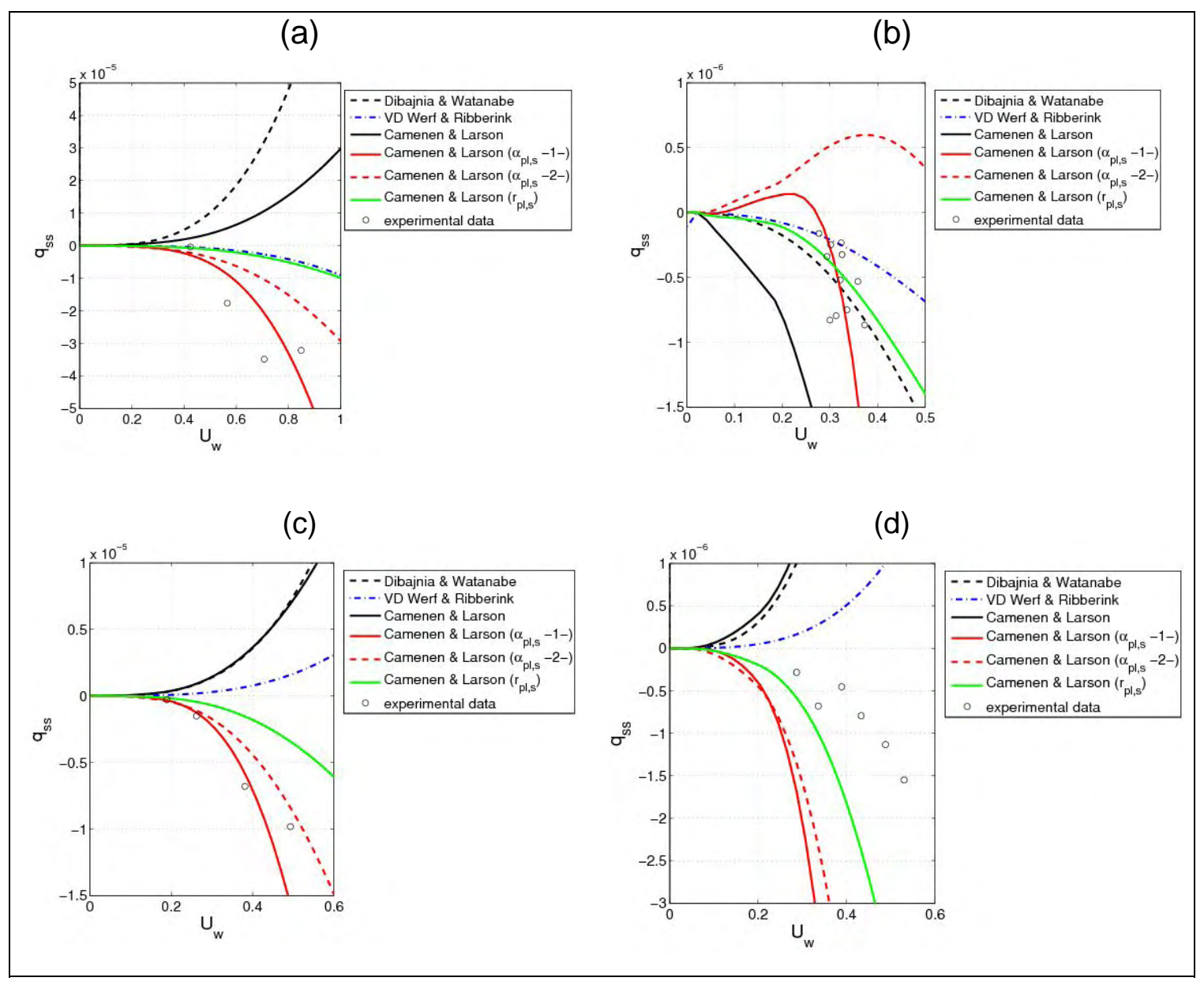

Figure 90. Influence of wave orbital velocity on sediment transport (details of input parameters for graphs a, b, c, and d are given in Table 38).

An increase in phase lag with an increase in orbital velocity clearly appears in the graphs. Equations 210 and 218 with $\alpha_{p l, s}$ yield the best behavior compared to the studied data with the exception of some data from Sato (1987) (Figure 90(d)) with irregular waves. For that case, as the Camenen 
and Larson (2007) formula seems to overestimate the reference concentration at the bottom and thus the quantity of sediments in suspension, an overestimation of the absolute sediment transport is observed. Another remark may be made based on the Grasmeijer (2002) data obtained in a flume where a weak undertow was measured. Three main factors play important roles for the direction of the sediment transport (direction of the mean flow, asymmetric waves opposite to the mean current, and phase lag of the sediment), and the formulas and measurements are sensitive to slight variations in each of these parameters.

Effect of wave period. The wave period is also an important factor for the phase lag and its effects on sediment transport: the shorter $T_{w}$ is, the larger the amount of sediment still in suspension after half a period. The delay in sediment settling before the change in the velocity direction strongly depends on the wave period. Table 39 and Figure 91 show the obtained results for selected measurements.

Table 39. Experiment conditions for studied cases on wave period effects (mean value is used for ripple height $H_{r}$ ).

\begin{tabular}{|l|l|l|l|l|l|l|l|}
\hline Case & Author(s) & $\begin{array}{l}d_{50} \\
(\mathrm{~mm})\end{array}$ & $\begin{array}{l}h \\
(\mathrm{~m})\end{array}$ & $\begin{array}{l}U_{c} \\
(\mathrm{~m} / \mathrm{sec})\end{array}$ & $\begin{array}{l}U_{\mathrm{w}} \\
(\mathrm{m} / \mathrm{sec})\end{array}$ & $\begin{array}{l}r_{w} \\
(-)\end{array}$ & $\begin{array}{l}H_{r} \\
(\mathrm{~mm})\end{array}$ \\
\hline a & Van der Werf and Ribberink & 0.35 & 1.10 & 0 & 0.56 & 0.26 & 8.2 \\
\hline b & Sato & 0.18 & 0.24 & 0 & 0.26 & 0.25 & 1.8 \\
\hline
\end{tabular}

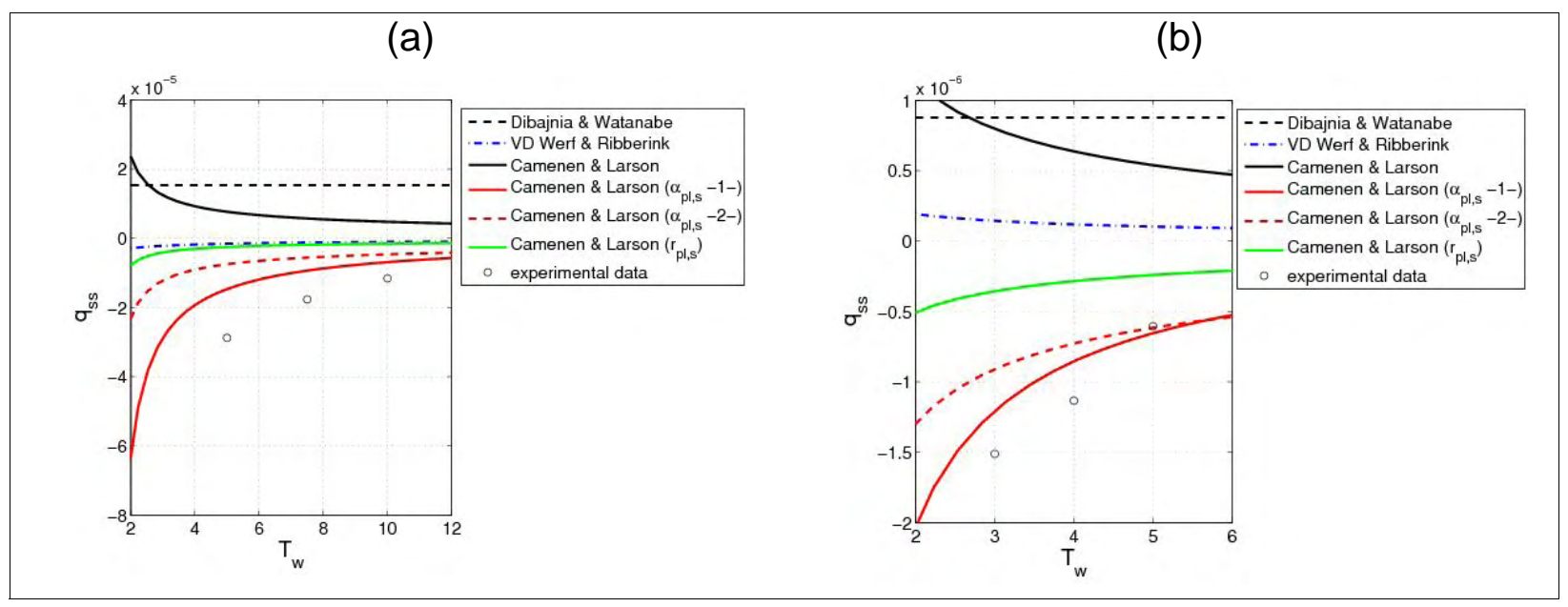

Figure 91. Influence of wave period on sediment transport (details of input parameters for graphs $\mathrm{a}$ and $\mathrm{b}$ are given in Table 39 ). 
The influence of the wave period is clearly seen in the Figure 91. Regarding the wave period, Equations 210 and 218 with $\alpha_{p l, s}$ yield the best behavior compared to the studied data. The other formulas appear to be independent of the wave period.

Effect of wave asymmetry. The wave asymmetry also plays an important role in the phase lag as it enhances the influence of the wave orbital velocity during the onshore half period: the larger $r_{w}$ is, the larger the amount of sediment put in suspension during the onshore half period. Table 40 and Figure 92 show the obtained results for selected experimental data.

Table 40. Experiment conditions for studied cases on wave asymmetry effects (mean value is used for ripple height $H_{r}$ ).

\begin{tabular}{|l|l|l|l|l|l|l|l|}
\hline Case & Author(s) & $\begin{array}{l}d_{50} \\
(\mathrm{~mm})\end{array}$ & $\begin{array}{l}h \\
(\mathrm{~m})\end{array}$ & $\begin{array}{l}U_{\mathrm{c}} \\
(\mathrm{m} / \mathrm{sec})\end{array}$ & $\begin{array}{l}U_{\mathrm{w}} \\
(\mathrm{m} / \mathrm{sec})\end{array}$ & $\begin{array}{l}T_{w} \\
(\mathrm{sec})\end{array}$ & $\begin{array}{l}H_{r} \\
(\mathrm{~mm})\end{array}$ \\
\hline a & Sato & 0.18 & 0.42 & 0 & 0.48 & 3.0 & 1.4 \\
\hline b & Sato & 0.18 & 0.35 & 0 & 0.23 & 5.0 & 1.9 \\
\hline
\end{tabular}

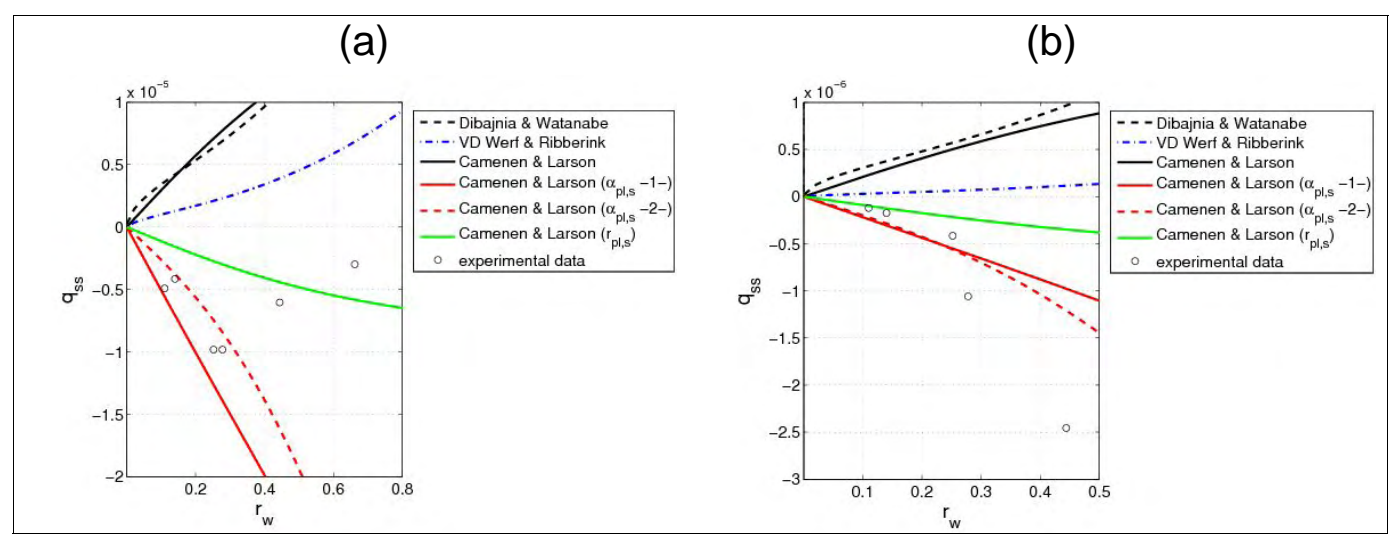

Figure 92. Influence of wave asymmetry on sediment transport (details of input parameters for graphs a and $b$ are given in Table 40).

The influence of wave asymmetry is clearly observed in Figure 92. If, for a given wave orbital velocity some phase lag occurs, an increasing asymmetry of the wave increases the lag because more sediment is put into suspension during the first half period and transported during the second half period. Again, Equations 210 and 218 with $\alpha_{p l, s}$ yield the best behavior compared to the studied data. However, for some specific data (Figure 92), it appears that the wave asymmetry, which also induces an increasing onshore transport if no phase lag occurs, does not increase the phase lag 
further. The primary effect of $r_{w}$ (increasing onshore velocity, and thus onshore transport) seems to slowly prevail over the secondary effect (increasing suspension of sediment during the first half period, and thus possibly phase-lag effects). This behavior appears not to be described by the proposed formula.

\section{Concluding remarks on phase-lag effects}

A study of the phase-lag effects on the suspension due to a rippled bed was presented here. As shown by Van der Werf and Ribberink (2004), the vortex suspension parameters $P_{W R}$ appears to be a fundamental quantity for estimating phase-lag effects due to the ripples. The modification of the suspended load formula presented by Camenen and Larson (2007) was presented using three different methods. The first method introduces a factor $r_{p l, s}$ which can be used for any other semi-empirical formula for the suspended load. The simple conceptual model to estimate $r_{p l, s}$ was calibrated using an empirical function of $P_{W R}$ for the coefficient $\phi_{p l}$. This formula coupled with the original Camenen and Larson (2007) formula yields accurate results, even if the phase-lag effects are underestimated. The two other methods are based on a modification of the Camenen and Larson (2007) formula, through the introduction of the velocity $U_{c w}$,net instead of the mean velocity $U_{c}$. The velocity $U_{c w, n e t}$ corresponds to the summation of an onshore and offshore velocity (if there are no waves, $U_{c w, n e t}=U_{c}$ ). A coefficient $\alpha_{p l, s}$ is then introduced in $U_{c w, \text { net }}$ to decrease the characteristic onshore velocity and increase the characteristic offshore velocity if a phase lag occurs. The parameter $\alpha_{p l, s}$ was first found analytically using the conceptual model, but it was also directly estimated through calibration against data. Both expressions obtained for $\alpha_{p l, s}$ significantly improved the suspended load model for a rippled bed. Compared to the studied semi-empirical formulas, the present model yields the best overall predictions. 


\section{A Unified Sediment Transport Formula for Coastal Inlet Application}

\section{Summary of total load formula}

In the following, a summary is given of the governing equations in the sediment transport model in concise form. Previous presentation of the model included many aspects of the equations that were developed and tested, which could make it difficult for the reader to extract the most suitable equations needed for computations.

\section{Bed-load transport}

Camenen and Larson (2005a, b) developed a formula for the bed-load transport based on the Meyer-Peter and Müller (1948) formula. The bedload transport $\left(q_{s b}\right)$ may be expressed as follows:

$$
\begin{aligned}
& \frac{q_{s b w}}{\sqrt{(s-1) g d_{50}^{3}}}=a_{w} \frac{\theta_{c w, n e t}}{\sqrt{\left|\theta_{c w, n e t}\right|}} \theta_{c w, m} \exp \left(-b \frac{\theta_{c r}}{\theta_{c w}}\right) \\
& \frac{q_{s b n}}{\sqrt{(s-1) g d_{50}^{3}}}=a_{n} \frac{\theta_{c n}}{\sqrt{\left|\theta_{c n}\right|}} \theta_{c w, m} \exp \left(-b \frac{\theta_{c r}}{\theta_{c w}}\right)
\end{aligned}
$$

where the subscripts $w$ and $n$ correspond, respectively, to the wave direction and the direction normal to the waves, $s$ is the relative density between sediment $\left(\rho_{s}\right)$ and water $(\rho), g$ the acceleration due to gravity, $d 50$ the median grain size, $a_{w}, a_{n}$, and $b$ are empirical coefficients (to be discussed), $\theta_{c w, m}$ the mean Shields parameter, $\theta_{c w}$ the maximum Shields parameter due to wave-current interaction, $\theta_{c n}=0.5 f_{c} U_{c}^{2} \sin \varphi|\sin \varphi| /\left((s-1) g d_{50}\right)$, where $f_{c}$ is the current related friction factor, $U_{c}$ the steady current velocity, and $\varphi$ the angle between the wave and the current direction. To simplify the calculations, the mean and maximum Shields parameter due to wave-current interaction is obtained by vector addition: $\theta_{c w, m}=\left(\theta_{c}^{2}+\theta_{w, m}^{2}+2 \theta_{w, m} \theta_{c} \cos \varphi\right)^{1 / 2}$ and $\theta_{c w}=\left(\theta_{c}^{2}+\theta_{w}^{2}+2 \theta_{w} \theta_{c} \cos \varphi\right)^{1 / 2}$, where $\varphi$ is the angle between the mean current direction and the wave incidence direction, and $\theta_{c}, \theta_{w, m}$, and $\theta_{w}$ 
are the current, mean wave, and maximum wave Shields number, and $\theta_{w, m}$ $=0.5 \theta_{w}$ for a sinusoidal wave profile.

The net sediment transporting velocity in Equation 225 is given by:

$$
\theta_{c w, \text { net }}=\left(1-\alpha_{p l, b}\right) \theta_{c w, \text { onshore }}+\left(1+\alpha_{p l, b}\right) \theta_{c w, \text { offshore }}
$$

where $\theta_{c w, o n s h o r e ~}$ and $\theta_{c w, o f f s h o r e}$ are the mean values of the instantaneous shear stress over the two half periods $T_{w c}$ and $T_{w t}\left(T_{w}=T_{w c}+T_{w t}\right.$, in which $T_{w}$ is the wave period), and $\alpha_{p l, b}$ a coefficient accounting for the phase lag (Camenen and Larson 2006). In the same way as for the Dibajnia and Watanabe (1992) formula, the mean values of the instantaneous shear stress over a half period are defined as follows (Figure 93):

$$
\begin{aligned}
& \theta_{c w, \text { onshore }}=\frac{1}{T_{w c}} \int_{0}^{T_{w c}} \frac{1 / 2 f_{c w}\left[u_{w}(t)+U_{c} \cos \varphi\right]^{2}}{(s-1) g d_{50}} d t \\
& \theta_{c w, \text { offshore }}=-\frac{1}{T_{w t}} \int_{T_{w c}}^{T_{w}} \frac{1 / 2 f_{c w}\left[u_{w}(t)+U_{c} \cos \varphi\right]^{2}}{(s-1) g d_{50}} d t
\end{aligned}
$$

where $u_{w}(t)$ is the instantaneous wave orbital velocity, $t$ the time, and $f_{c w}$ the friction coefficient due to wave-current interaction introduced by Madsen and Grant (1976):

$$
f_{c w}=X_{v} f_{c}+\left(1-X_{v}\right) f_{w}
$$

with $X_{v}=\left|U_{c}\right| /\left(\left|U_{c}\right|+U_{w}\right)$, where $U_{w}$ is the average of the peak velocities during the wave cycle (the root-mean-square value is used for random waves).

Based on comparison with an extensive data set (Camenen and Larson 2005b), the following relationship is proposed for the transport coefficient $a_{w}$ :

$$
a_{w}=6+6 Y
$$




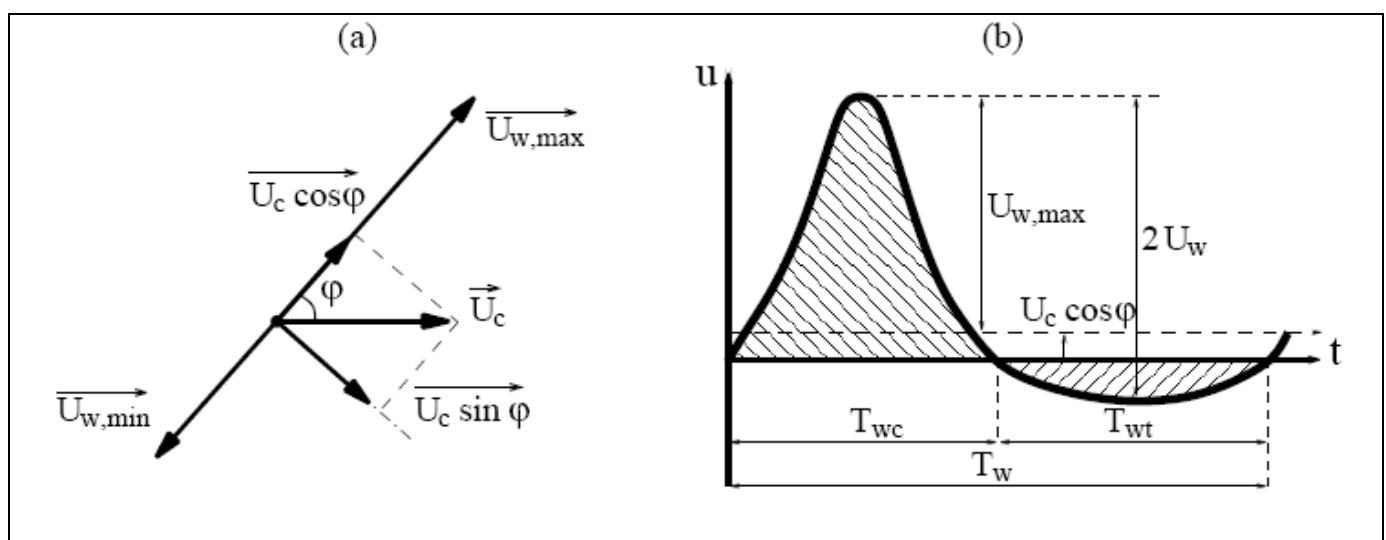

Figure 93. Definition of current and wave direction and velocity variation at bed in direction of wave propagation.

in which $Y=\theta_{c} /\left(\theta_{c}+\theta_{w}\right)$. The coefficient perpendicular to the waves, where only the current transport sediment, is set to $a_{n}=12$, and the coefficient in the term describing initiation of motion is $b=4.5$ (Equation 225). The phase lag is introduced through the coefficient $\alpha_{p l, b}=\alpha_{c}-\alpha_{t}$ following Camenen and Larson (2006):

$$
\alpha_{j}=\frac{v^{0.25} U_{c w, j}^{0.5}}{W_{s} T_{j}^{0.75}} \exp \left[-\left(\frac{U_{w, c r, s f}}{U_{c w, j}}\right)^{2}\right]
$$

where $U_{w, c r, s f}$ is the critical wave orbital velocity for the inception of sheet flow (Equation 57), and $U_{c w, j}$ is the root-mean-square value of the velocity (wave and current) over the half period $T_{w j}$, and the subscript $j$ should be replaced either by $c$ (crest or onshore) or $t$ (trough or offshore) (Figure 93):

$$
\begin{aligned}
& U_{c w, \text { onshore }}^{2}=\frac{1}{T_{w c}} \int_{0}^{T_{w c}}\left[u_{w}(t)+U_{c} \cos \varphi\right]^{2} d t \\
& U_{c w, o f f s h o r e}^{2}=\frac{1}{T_{w t}} \int_{T_{w c}}^{T_{w}}\left[u_{w}(t)+U_{c} \cos \varphi\right]^{2} d t
\end{aligned}
$$

\section{Suspended load transport}

In determining the suspended load $q_{s s}$, following the simplified approach by Madsen (1993) and Madsen et al. (2003), the vertical variation in the horizontal velocity was neglected and an exponential-law profile assumed for the sediment concentration. Thus, the suspended sediment load may be obtained from (Camenen et al. 2005; Camenen and Larson 2007): 


$$
\left\{\begin{array}{l}
q_{s s w}=U_{c, n e t} c_{R} \frac{\varepsilon}{W_{s}}\left[1-\exp \left(-\frac{W_{s} h}{\varepsilon}\right)\right] \\
q_{s s n}=U_{c} \sin \varphi c_{R} \frac{\varepsilon}{W_{s}}\left[1-\exp \left(-\frac{W_{s} h}{\varepsilon}\right)\right]
\end{array}\right.
$$

where $h$ is the water depth, $U_{c, \text { net }}$ the net mean current over a wave period, $c_{R}$ the reference concentration at the bottom, $W_{s}$ the sediment fall speed, and $\varepsilon$ the sediment diffusivity. In calculating the integral, the ratio $W_{s} h / \varepsilon$ may often be assumed large, implying that the exponential term is close to zero. However, such an assumption that integrating to infinity or to $h$ produces about the same result may not be valid if a strong mixing by wave breaking is present. The bed reference concentration is obtained from:

$$
c_{R}=A_{c R} \theta_{c w, m} \exp \left(-4.5 \frac{\theta_{c r}}{\theta_{c w}}\right)
$$

The coefficient $A_{c R}$ is written as follows:

$$
A_{c R}=3.510^{-3} \exp \left(-0.3 d_{*}\right)
$$

where $d_{*}=\sqrt[3]{(s-1) g / v^{2}} d_{50}$ is the dimensionless grain size. A multiplying factor is introduced if plunging breakers occurs:

$$
C_{R b}=1.0+\tanh \left(50\left(\xi_{\infty}-0.15\right)\right) \text { if } \xi_{\infty}>0.15
$$

The sediment diffusivity is related to the energy dissipation:

$$
\varepsilon=\left(\frac{D}{\rho}\right)^{1 / 3} h
$$

in which $D$ is the total effective dissipation:

$$
D=k_{b}^{3} D_{b}+k_{c}^{3} D_{c}+k_{w}^{3} D_{w}
$$

where the energy dissipation from wave breaking $\left(D_{b}\right)$ and from bottom friction due to current $\left(D_{c}\right)$ and waves $\left(D_{w}\right)$ were simply added, and $k_{b}, k_{c}$, and $k_{w}$ are coefficients (Equations 175, 166, and 167, respectively). The 
coefficient $k_{b}$ corresponds to an efficiency coefficient, whereas $k_{c}$ and $k_{w}$ are related to the Schmidt number. Assuming a parabolic profile for the vertical sediment diffusivity, its mean value over the depth (for a steady current or waves, respectively) may be written as follows:

$$
\varepsilon_{c / w}=\left(\frac{D_{c / w}}{\rho}\right)^{1 / 3} h=\frac{\sigma_{c / w}}{6 C_{w}} \kappa u_{w_{c} / w} h
$$

where $\sigma_{c / w}$ is the Schmidt number or ratio between the vertical eddy diffusivity of the particles $\varepsilon_{v}$ and the vertical eddy viscosity $v_{v}$, and $u_{*_{c} / w}$ is the shear velocity due to a current or to waves only, respectively, and $C_{w}$ is an integration constant that is 1 for the case of a steady current and $\pi / 2$ for a sinusoidal wave. The following expression for the Schmidt number was proposed:

$$
\sigma_{c / w}= \begin{cases}A_{1}+A_{2} \sin ^{2}\left(\frac{\pi}{2} \frac{W_{s}}{u_{*_{c} / w}}\right) & \text { if } \frac{W_{s}}{u_{*_{c} / w}} \leq 1 \\ 1+\left(A_{1}+A_{2}-1\right) \sin ^{2}\left(\frac{\pi}{2} \frac{u_{*_{c} / w}}{W_{s}}\right) & \text { if } \frac{W_{s}}{u_{*_{c} / w}}>1\end{cases}
$$

where $A_{1}=0.4$ and $A_{2}=3.5 \mathrm{in}$ case of a steady current alone and $A_{1}=0.15$ and $A_{2}=1.5$ in case of waves only. For wave and current interaction, a weighted value is employed for the Schmidt number:

$$
\sigma_{c w}=Y \sigma_{c}+(1-Y) \sigma_{w}
$$

The net mean current is defined in a similar way to the net Shields parameter for the bed load in order to take into account sediment transport due to asymmetric waves, as well as possible phase lag between the suspended concentration and the velocity:

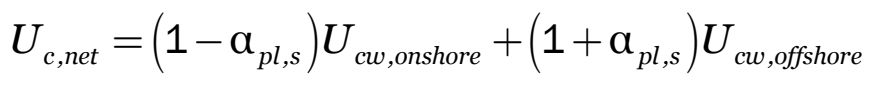

where $\alpha_{p l, s}$ is a coefficient for the phase lag on the suspended load (Equation 221). For a steady current, $U_{c, \text { net }}=U_{c}$. 


\section{Bottom slope}

The bottom slope may influence the sediment transport, especially if it is close to the critical value given by the internal friction angle at saturated conditions of the sediment. To take into account the local slope, the transport rate $\left(q_{s}\right)$ may be multiplied with a function containing the local slope and a coefficient,

$$
q_{s}^{*}=q_{s}\left(1-\beta \frac{\partial z_{b}}{\partial_{s}}\right)
$$

where $\beta$ is a coefficient for the bottom slope $(0.5<\beta<2)$, and $\partial z b / \partial s$ is the local slope. Following Bailard (1981), the coefficient $\beta$ depends on the sediment transport mode:

$$
\begin{array}{ll}
\beta_{b}=\frac{1}{\tan \phi} \approx 1.5 & \text { (bed load) } \\
\beta_{s}=\frac{\varepsilon_{s}<|\vec{u}|>}{W_{s}} \quad \text { (suspended load) }
\end{array}
$$

where $\varepsilon_{s}=0.02$ is the suspended-load efficiency as given by Bailard (1981), and $\vec{u}$ is the instantaneous velocity vector (wave and current combined).

\section{Velocity profiles for varying slope}

Variations in the velocity profile can influence both bed load and suspended load transport because the characteristic velocity in the lower part of the water column, where the concentration is larger, may be significantly reduced. Coles (1956) showed that velocity profiles in a nonuniform flow can be described by a linear combination of logarithmic profiles representing the law of the wall and a perturbation profile representing the influence of pressure gradients:

$$
u(z)=A_{1} u_{h} \ln \left(\frac{z}{z_{0}}\right)+A_{2} u_{h} F\left(\frac{z}{h}\right)
$$

in which $u_{h}$ is flow velocity at the water surface $(z=h), z_{o}$ the zero-velocity level ( $z_{o}=0.03 k_{s}$ from Van Rijn and Tan 1985), and $A_{1}$ and $A_{2}$ are dimensionless variables. Van Rijn and Tan (1985) proposed the following perturbation profile: 


$$
F\left(\frac{z}{h}\right)=2\left(\frac{z-z_{0}}{h-z_{0}}\right)^{n}-\left(\frac{z-z_{0}}{h-z_{0}}\right)^{2 n}
$$

Applying the boundary condition $u(z)=u_{h}$ for $z=h$, introducing the discharge integrated over the width $Q=B h U_{c}=B \int_{z_{0}}^{h} u d z$, where $B$ is the width of the flow, and assuming that the middepth velocity is approximately the same as for a uniform flow, a relationship for $n$ can be numerically obtained:

$$
0.16 n^{2}-0.29 n+1.02 \approx \frac{\ln \left(h / z_{0}\right)-1}{\ln \left(0.5 h / z_{0}\right)}
$$

Figure 94 shows that this method is capable of representing a wide range of velocity profiles including those with flow reversal.

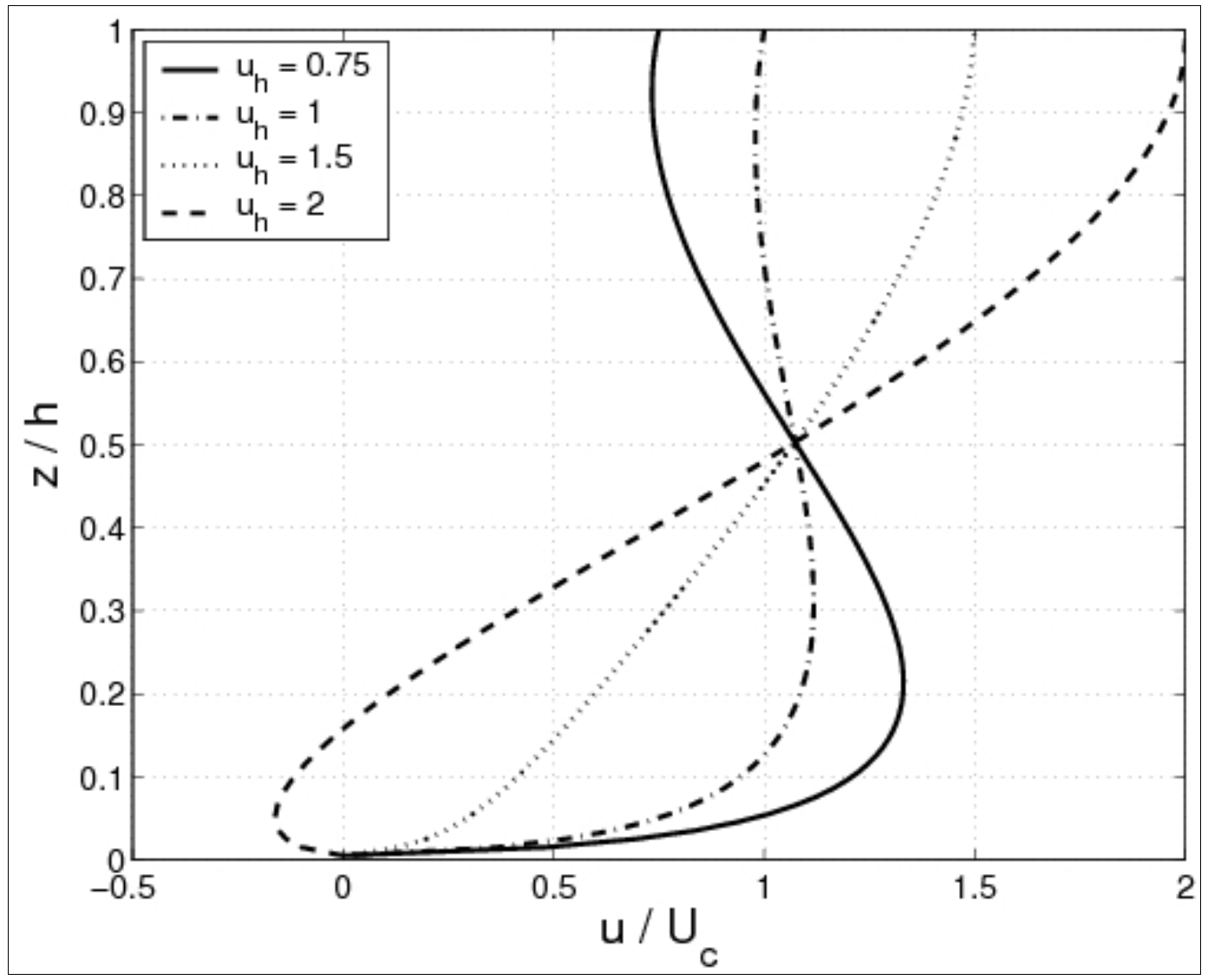

Figure 94. Velocity profiles according to Equations 244,245 , and $246\left(U_{c}=0.4 \mathrm{~m} / \mathrm{sec}\right.$, $h=0.2 \mathrm{~m}$, and $\left.z_{o}=0.001 \mathrm{~m}\right)$. 
Van Rijn and Tan (1985) proposed a first-order differential equation to solve for the spatial variation of the water surface velocity $u_{h}$, which yields an exponential adjustment of the surface velocity with respect to the equilibrium surface velocity $u_{h, e}$, as follows:

$$
\frac{d u_{h}}{d h}=\alpha_{1} \frac{u_{h, e}}{h}-\alpha_{2} \frac{u_{h}}{h}-\alpha_{3} \frac{u_{h}}{B}
$$

The coefficients $\alpha_{1}$ and $\alpha_{2}$ have been found to depend on the local bottom slope with values determined by comparison to data from several experiments, and $\alpha_{3}$ takes into account lateral variations:

$$
\begin{aligned}
& \alpha_{1}=0.28+0.11 \tanh [6(d h / d x)-0.15] \\
& \alpha_{2}=0.235+0.065 \tanh [17(d h / d x-0.035)] \\
& \alpha_{3}=0.1 \tanh [10(d B / d x)]
\end{aligned}
$$

Equation 247 can be solved numerically by a Runge-Kutta method, with the surface velocity $u_{h, 0}$ as the boundary condition.

\section{Application to coastal inlet studies}

Longshore sediment transport forms the main input required for many coastal engineering projects such as dredging of inlet navigation channels, assessment of beach evolution in the vicinity of jetties and groins, and the evolution and stability of inlets, breaches, and estuaries. For coastal inlets, complex interactions occur among the longshore current, tidal current in the inlet, and waves, which may induce many types of phenomena related to sediment transport (Figure 2).

\section{Validation of longshore sediment transport}

To validate the present formula for the case of longshore sediment transport, two data sets were employed. Bayram et al. (2001) discussed the Sandy Duck experiments carried out at the U.S. Army Corps of Engineers Field Research Facility in Duck, NC (for a summary of the field experiments, see Miller 1998, 1999). During these experiments, the cross-shore distribution of the time-averaged longshore current and sediment concentration were measured, from which the transport rate could be estimated for six cases from 1996 to 1998. Wang et al. (2002) performed four sets of experiments in a large wave basin (Large-scale Sediment Transport 
Facility - LSTF) at the Coastal and Hydraulics Laboratory, Vicksburg, MS. The longshore sediment transport rate was recorded on a sandy beach exposed to random waves breaking at an incident wave angle, in one experiment as plunging breakers and in the other as spilling breakers. In the LSTF experiments, the hydrodynamics and concentration profiles were recorded at many locations across the profile. The beach profiles for the spilling breaker case were similar to the plunging case apart from the shape of the offshore bar, which was less pronounced for the spilling breaker case, implying less intensive wave breaking (and energy dissipation) in this region.

Table 41 presents the main hydrodynamic conditions together with the median grain size for five of the experimental cases from Sandy Duck and the LSTF employed here for validating the sediment transport model. The main difference between the cases in the LSTF experiment was the breaker type, whereas the initial bathymetry was the same. For the Sandy Duck experiments, the bathymetry varied between the cases, where a clear bar was observed during the early cases and a terrace-shaped beach for later cases.

Table 41. Experiment conditions for studied cases on longshore sediment transport.

\begin{tabular}{|l|l|l|l|l|l|l|}
\hline Case & Characteristics & $\begin{array}{l}\xi_{\infty} \\
(-)\end{array}$ & $\begin{array}{l}d_{50} \\
(\mathrm{~mm})\end{array}$ & $\begin{array}{l}H_{w_{\infty}} \\
(\mathrm{m})\end{array}$ & $\begin{array}{l}T_{w} \\
(\mathbf{s})\end{array}$ & $\begin{array}{l}\alpha_{w} \\
(\mathrm{deg})\end{array}$ \\
\hline LSTF Case 1 & Spilling breakers & 0.30 & 0.2 & 0.25 & 1.5 & 30 \\
\hline LSTF Case 6 & Plunging breakers & 0.75 & 0.2 & 0.19 & 3.0 & 30 \\
\hline Sandy Duck 12/03/96 & Barred beach & 0.20 & 0.20 & 3.1 & 13 & 5 \\
\hline Sandy Duck 31/03/97 & Barred beach & 0.15 & 0.18 & 1.4 & 8 & 5 \\
\hline Sandy Duck 04/02/98 & Terrace beach & 0.20 & 0.18 & 2.3 & 13 & 5 \\
\hline
\end{tabular}

For these experiments, mainly the suspended load was estimated (the transport rate was derived from time-averaged sediment concentration and velocities). Furthermore, as the evolution of the wave asymmetry $r_{w}$ was not available, it was estimated using the formula by Dibajnia et al. (2001) (Figures 95(a), 96(a), 97(a) and 98(a); the first of the three plots in each figure). Similarly, for the LSTF data set, the cross-shore current (undertow) was not available, and it was estimated using the expression proposed by Svendsen (1984) (Figures 95(a), 96(a), 97(a), and 98(a); the second of the three plots in each figure). These calculations are a source of error in the estimation of the suspended load. 


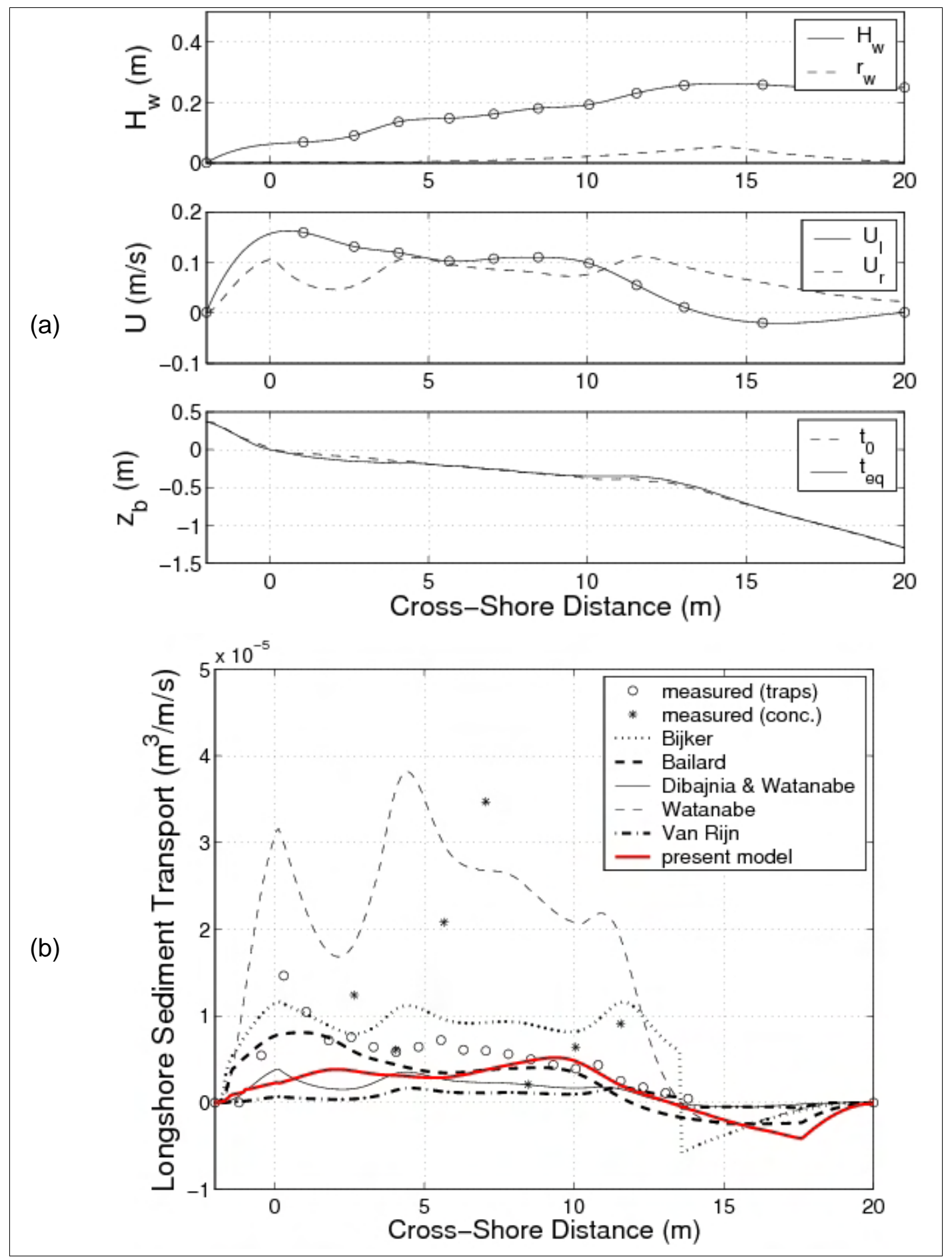

Figure 95. Cross-shore variations in hydrodynamic parameters and beach profile for an LSTF experimental case (Test 1 - spilling breakers) together with (a) measured longshore suspended sediment transport, and (b) calculated transport using six studied formulas. 


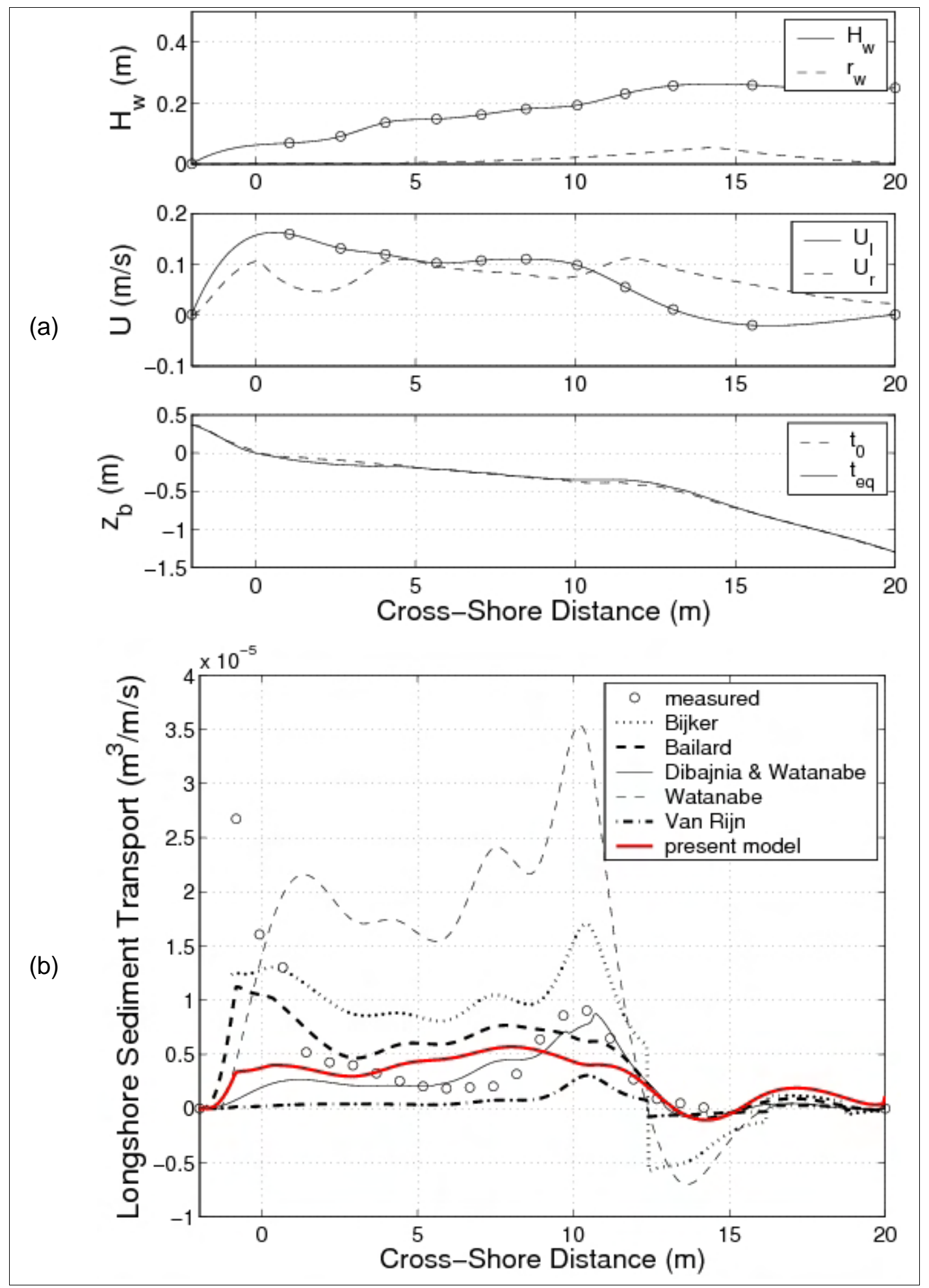

Figure 96. Cross-shore variations in hydrodynamic parameters and beach profile for an LSTF experimental case (Test 6 - plunging breakers) together with (a) measured longshore suspended sediment transport, and (b) calculated transport using six studied formulas. 
(a)
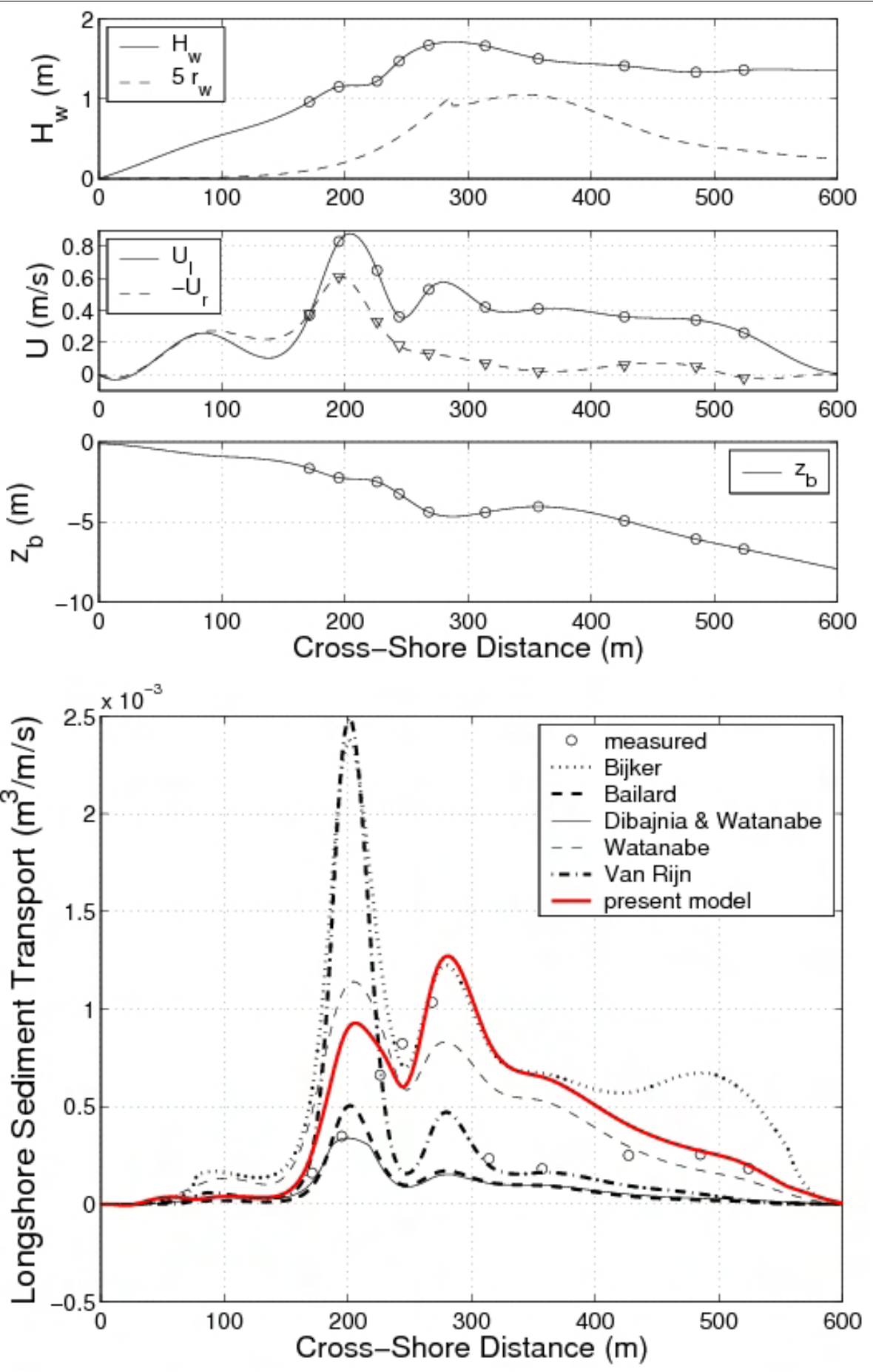

Figure 97. Cross-shore variations in hydrodynamic parameters and beach profile for

Sandy Duck experiment (31 March 1997) together with (a) measured longshore suspended sediment transport, and (b) calculated transport using six studied formulas. 


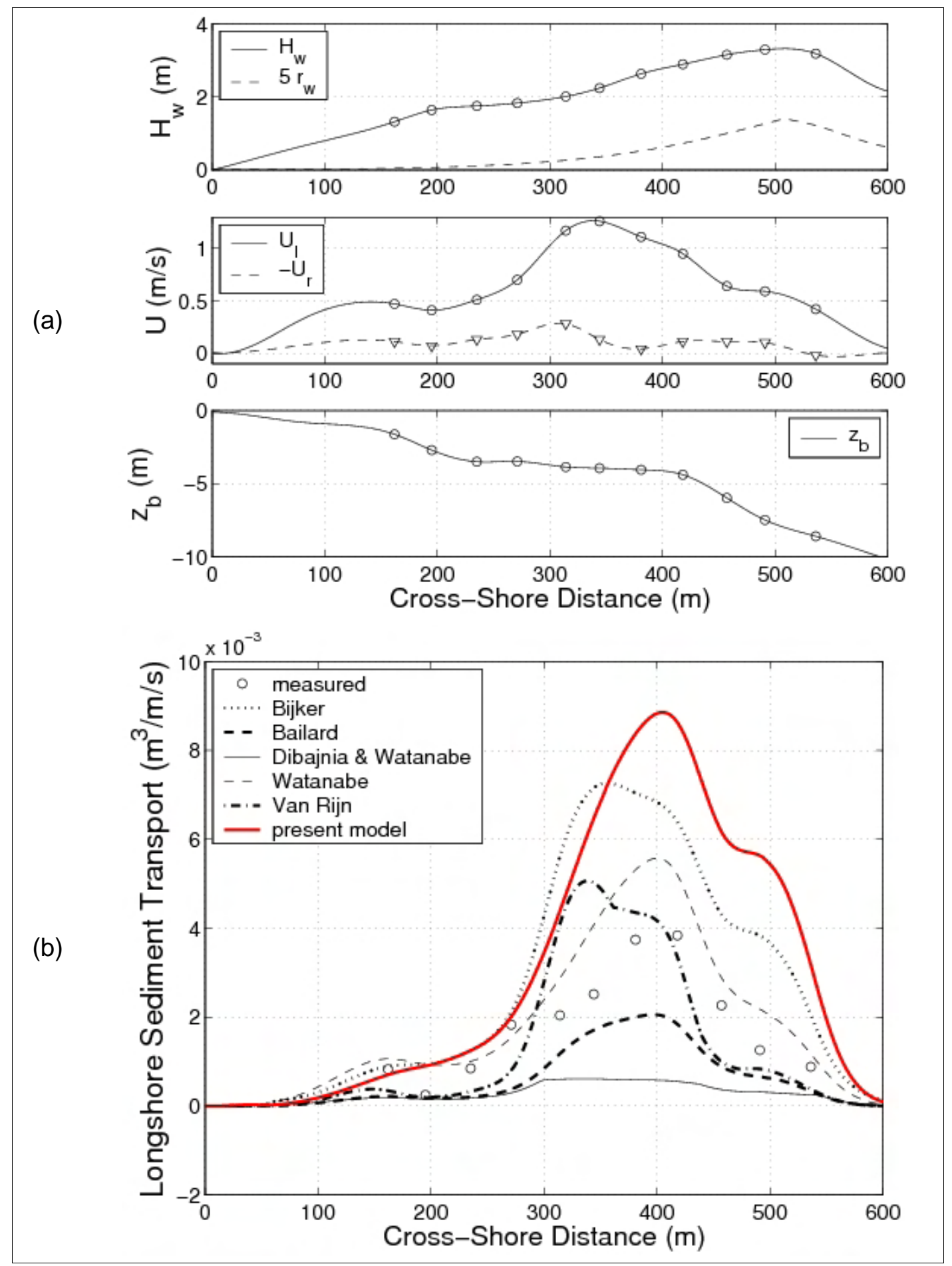

Figure 98. Cross-shore variations in hydrodynamic parameters and beach profile for Sandy Duck experiment (4 February 1998) together with (a) measured longshore suspended sediment transport, and (b) calculated transport using six studied formulas. 
The ripple characteristics were not measured, and they had to be estimated using a predictive formula. As previously discussed, this leads to large uncertainty in estimation of the Shields parameter. For the LSTF

Figure 95 shows the result obtained for the LSTF experiment Case 1 presented (case with spilling breakers) for the longshore sediment transport. Apart from the Watanabe and Isobe (1992) formula, which largely overestimate the results, and the Van Rijn (1989) formula, which tends to underestimate the results, all studied formulas yields similar results that lie close to the observations. However, all formulas underestimate the sediment transport in the swash zone, which is a region not properly described by the formulas. The physical mechanisms for the longshore sediment transport in the swash zone is different compared to the surf zone, and other formulas are necessary to describe the sediment transport rates in this region.

Figure 96 is the result obtained for the LSTF experimental Case 6 with plunging breakers. In the surf zone, most formulas tend to overestimate the transport rates although the location of the peak is well predicted. The Bailard (1981) formula and the present formula overall yield the correct magnitude, even if the peak of sediment transport in the zone of incipient breaking is underestimated and not as pronounced as in the measurements.

Figures 97 and 98 are representative results obtained for the Sandy Duck experiments regarding the longshore sediment transport rate shown. The spread in calculations is larger than for the LSTF data because larger uncertainty exists in the measurements of the current profiles and especially in the estimation of the concentration profiles. It appears that the main difference between the formulas is the magnitude. If the present formula, the Bijker (1968) formula, and the Watanabe and Isobe (1992) formula tend to overestimate the suspended load, the Bailard formula and the Dibajnia and Watanabe (1992) formula tend to underestimate the rates. It appears that the Bijker (1968) and Van Rijn (1989) formulas are too sensitive to the current magnitude and tend to overestimate the sediment transport at the peak of the longshore current, even if the wave height is smaller than at the breaker line (Figure 97). 
Table 42 presents the statistical results for all the formulas and both data sets. The percentage of values obtained with an error less than factor 2 or 5 (designed as $P \times 2$ or $P \times 5$ ) as well as the mean value and the standard deviation of the function $f\left(q_{s s}\right)=\log \left(q_{s s, \text { pred }} / q_{s s, \text { meas }}\right)$ are presented in the table. The Bailard (1981) formula gives the best results for the Sandy Duck data, but yields poorer results for the LSTF data. The Watanabe and Isobe (1992) formula yields good results for the Sandy Duck experiments, but poor agreement for the LSTF data. These two formulas as well as the Dibajnia and Watanabe (1992) formula seem to be sensitive to the scale of data collection. This is a result of the formulas not being functions of the total shear stress (which varies with the scale of the experiment), but only of the velocity profile (i.e., Dibajnia and Watanabe (1992) and Bailard 1981) formula) or are too simple to include all the parameters governing the bed load and suspended load (i.e., Watanabe and Isobe (1992) formula). For the cases studied here regarding longshore sediment transport, the Bijker (1968) and the present formula yield the overall best results. The Van Rijn (1989) formula generally underestimates the results and appears to be more sensitive to the steady current magnitude.

Table 42. Predictive capability of different transport formulas for longshore suspended load transport for LSTF and Sandy Duck experiments.

\begin{tabular}{|l|l|l|l|l|l|}
\hline Author(s) & $P \times 2(\%)$ & $P \times 5(\%)$ & Mean $\left(f\left(q_{s s}\right)\right)$ & Std $\left(f\left(q_{s s}\right)\right)$ \\
\hline \multicolumn{5}{|c|}{ LSTF Data } \\
\hline Bijker (1968) & 48 & 78 & 1.0 & 1.5 \\
\hline Bailard (1981) & 60 & 77 & 0.35 & 1.7 \\
\hline Van Rijn (1989) & 08 & 37 & -1.4 & 1.9 \\
\hline Watanabe and Isobe (1992) & 11 & 60 & 1.4 & 1.3 \\
\hline Dibajnia and Watanabe (1992) & 35 & 75 & -0.45 & 1.5 \\
\hline Present formula & 55 & 75 & 0.10 & 1.7 \\
\hline \multicolumn{2}{|l|l|l|l|}{} & 85 & 0.8 & 0.5 \\
\hline Bijker (1968) & Sandy Duck Data & -1.3 & 0.8 \\
\hline Bailard (1981) & 33 & 68 & -1.3 & 1.1 \\
\hline Van Rijn (1989) & 30 & 56 & 0.1 & 0.9 \\
\hline Watanabe and Isobe (1992) & 20 & 91 & -1.4 & 0.6 \\
\hline Dibajnia and Watanabe (1992) & 61 & 64 & 0.4 & 0.9 \\
\hline Present formula & 17 & 86 & \\
\hline
\end{tabular}


Figure 99 plots the predictive results of the longshore sediment transport rate across the beach profile for both experiments using the present formula. It confirms the underestimation in the swash zone observed for the LSTF data as well as a slight underestimation in the zone of incipient breaking. Because of a larger uncertainty in the measurements and calculations for the Sandy Duck data, a larger discrepancy is observed for these data. In general, the formula overestimates the transport rates, which may be due to an overestimation of the sediment diffusivity for waves at a large water depth, as previously pointed out.

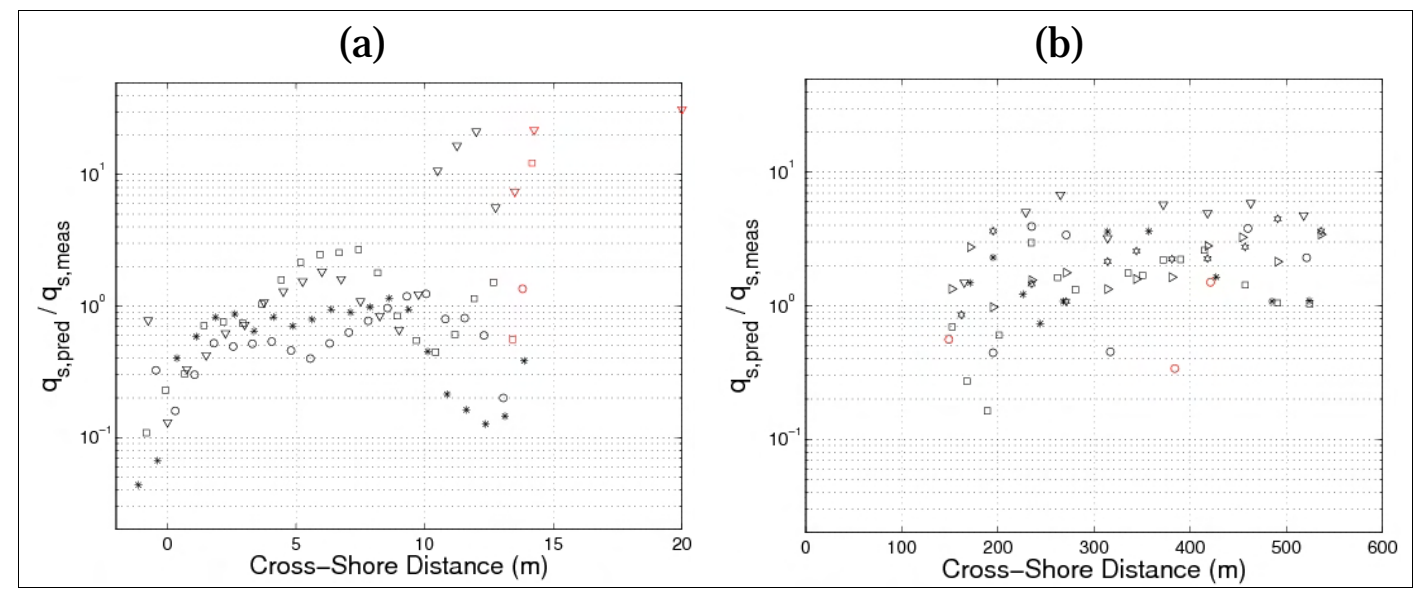

Figure 99. Predictive results for longshore sediment transport rate across beach profile using present formula for (a) LSTF data, and (b) Sandy Duck data.

\section{Validation of cross-shore sediment transport}

The cross-shore sediment transport rate could also be estimated for the Sandy Duck data. However, the sensitivity in the predictions by the formulas is much greater compared to the longshore transport rate. Figure 100 presents results obtained for one experimental case carried out on 12 March 1996. The Bijker (1968), Van Rijn (1989), and Watanabe and Isobe (1992) formulas induce a sediment transport that is in the same direction as the undertow, which means in the offshore direction. In contrast, the Bailard (1981), Dibajnia and Watanabe (1992) formulas, as well as the present formula, allows for transport in the opposite direction to the mean current if asymmetric waves are present. Thus, onshore sediment transport is often observed seaward of the surf zone. Because the transport rates derived from the measurements do not take into account the wave-induced sediment transport, it differs from the calculation results for the three latter formulas (for the other formulas, the estimated sediment transport rate is always in the direction of the mean current). 


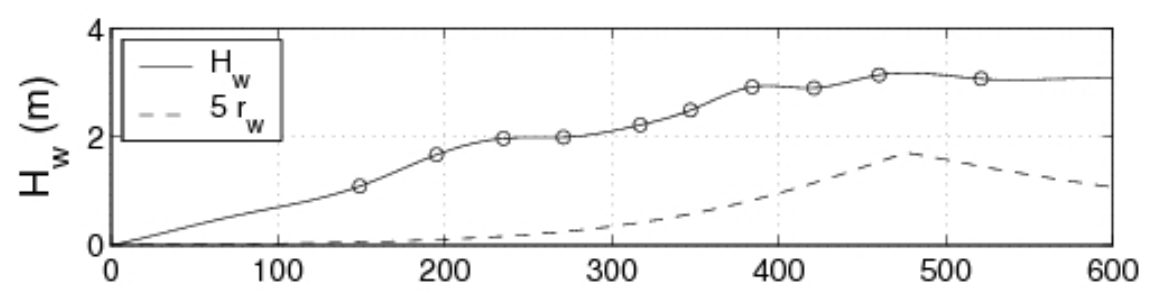

(a)
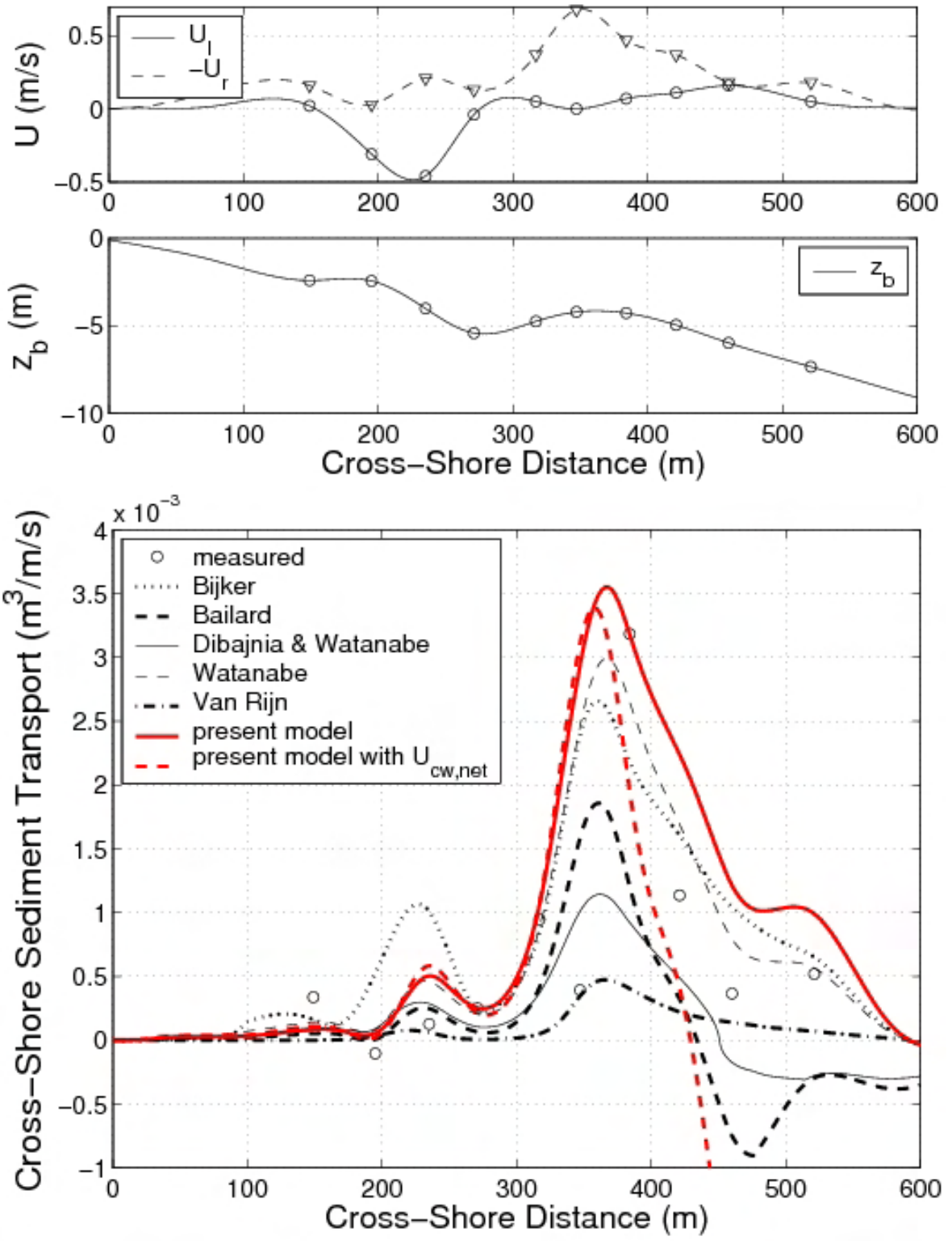

Figure 100. Cross-shore variations in hydrodynamic parameters and beach profile for (a) Sandy Duck experimental case (12 March 1996) together with measured cross-shore suspended sediment transport, and (b) calculated transport using six studied formulas. 
The present formula appears to be sensitive to the balance between the undertow and wave asymmetry at the seaward end of the surf zone (compare results when $U_{c}$ is used instead of $U_{c w, n e t}$ for the suspended load; see Figure 100).

Table 43 presents the statistical results for all the formulas compared regarding the Sandy Duck experiments on cross-shore transport. It appears for these cases that the predictive results are poorer than for the longshore sediment transport rate. The Watanabe and Isobe (1992) formula presents the best results, which is surprising because it was calibrated for longshore transport. However, as discussed previously, the measured suspended load includes transport by the mean current only, and other mechanisms are not included.

Table 43. Predictive capability of different transport formulas regarding suspended load transport in cross-shore direction for Sandy Duck experiments.

\begin{tabular}{|l|l|l|l|l|}
\hline Author(s) & $P \times 2(\%)$ & $P \times 5(\%)$ & Mean $\left(f\left(q_{s s}\right)\right)$ & Std $\left(f\left(q_{s s}\right)\right)$ \\
\hline Bijker (1968) & 14 & 36 & 2.3 & 1.2 \\
\hline Bailard (1981) & 23 & 50 & 0.05 & 1.6 \\
\hline Van Rijn (1989) & 24 & 53 & 0.15 & 1.7 \\
\hline Watanabe and Isobe (1992) & 47 & 68 & 0.8 & 1.0 \\
\hline Dibajnia and Watanabe (1992) & 35 & 61 & 0.02 & 1.2 \\
\hline Present formula & 33 & 65 & 1.1 & 1.1 \\
\hline
\end{tabular}

Figure 101 plots the prediction of the cross-shore sediment transport rate across the beach profile for the Sandy Duck experiments using the present formula with only the current-related suspended load included ( $U_{c}$ is used instead of $\left.U_{c w, n e t}\right)$. It confirms the general overestimation and dispersion previously observed for these data. If the wave-related sediment transport is included, the direction of the sediment transport is incorrectly estimated for several data points in the offshore. However, it may be a result of the formula including wave-related sediment transport, whereas the transport rates estimated from the measurements do not. 


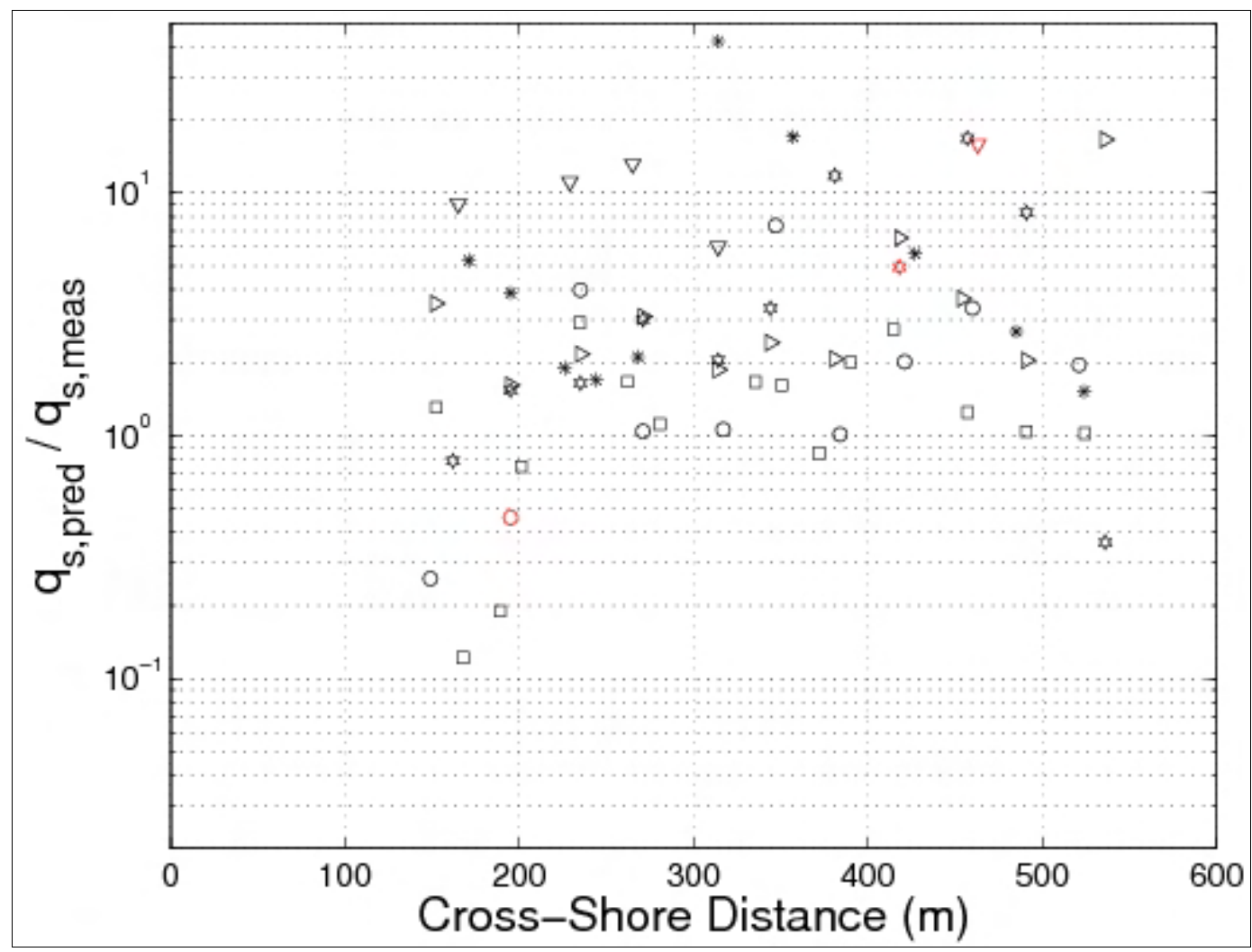

Figure 101. Comparison of cross-shore suspended load across profile line with present formula and Sandy Duck data.

An interesting data set was provided by Dohmen-J anssen and Hanes (2002). They measured bed load and suspended load transport in a large wave flume for sheet flow, and obtained results for the four cases presented in Table 44. Although a small undertow was present (opposite to the wave direction), the net sediment transport was directed onshore because of the asymmetric waves. The three formulas that assume the direction of the current to be the direction of the sediment transport (the Bijker (1968), Van Rijn (1989), and Watanabe and Isobe (1992) formulas) predict the wrong direction for the net total load. The Bailard (1981) formula predicts a correct direction for the sediment transport, but tends to overestimate the total load, especially the suspended load portion. 
Table 44. Predictive capability of total load sediment transport in cross-shore direction for sheet-flow experiments by Dohmen-Janssen and Hanes (2002).

\begin{tabular}{|l|l|l|l|l|l|}
\hline Author(s) & $P \times 2(\%)$ & $P \times 5(\%)$ & Mean $\left(f\left(q_{s s}\right)\right)$ & $\operatorname{Std}\left(f\left(q_{s s}\right)\right)$ & $q_{s b} / q_{s s}$ \\
\hline Bijker (1968) & $0 *$ & $0 *$ & -0.2 & 0.5 & 0.03 \\
\hline Bailard (1981) & 0 & 0 & 2.4 & 0.07 & 0.11 \\
\hline Van Rijn (1989) & 0 & $0 *$ & -0.3 & 0.2 & 1.3 \\
\hline Watanabe and Isobe (1992) & $0 *$ & $0 *$ & -0.4 & 0.4 & - \\
\hline Dibajnia and Watanabe (1992) & 100 & 100 & -0.15 & 0.3 & - \\
\hline Present Work & 75 & 100 & 0.5 & 0.3 & 0.4 \\
\hline * Opposite transport direction predicted.
\end{tabular}

Dohmen-J anssen and Hanes (2002) observed that, in case of sheet flow and nonbreaking waves, bed load was always dominant and only 10 percent of the total load was carried by the suspended load. The Bailard (1981) formula (as well as the Bijker (1968) formula) predicts that suspended load is dominant $\left(q_{s b} / q_{s s}=0.11\right)$. The Dibajnia and Watanabe (1992) formula, as it was calibrated for sheet-flow conditions, yields good results. Finally, the present formula also yields good results, although it tends to overestimate the suspended load.

\section{Comments on morphological evolution using total load formulas}

Total load formulas (bed load and suspended load) are commonly used in numerical models of morphological evolution. Based on the predictions of the local transport at fixed grid points, the gradients are computed and the sediment volume conservation equation is employed to determine the change in bed elevation. This approach implies that the transport rate can be determined at a point without considering the conditions at neighboring points. Such a simplification might be applicable in many situations, although it depends on the hydrodynamic, sedimentologic, and topographic conditions. If there is strong coupling between neighboring points, which might be the case for finer sediment and larger waves, governing equations that take into account horizontal exchange of material should be applied. The advection-diffusion (AD) equation is often utilized to model the horizontal exchange processes. Typically, the vertical exchange of material is assumed to occur rapidly, so that a fixed vertical concentration profile can be employed in the $\mathrm{AD}$ equation. If not, the total load formulas discussed in this paper is not applicable, and three-dimensional transport 
equations for the sediment must be employed. With respect to the coastal inlet environment, experience has shown that several types of morphological behavior might be difficult to simulate without calculating with the $\mathrm{AD}$ equation. 


\section{Conclusions}

This report presents an extensive study of the bed load and suspended load for the case of current and wave interaction. The unified formulation was developed for clastic material in sand range or coarser, and the formulas transition smoothly among differing degrees of waves and currents and between breaking and nonbreaking waves.

A formula for bed-load sediment transport was developed and presented that includes interaction between waves and current. This formula is based on the assumption that the sediment transport is proportional to the total Shields parameter to the power $3 / 2$. For purely oscillatory flows, the mean Shields parameter for each half period (for $u>0$ and for $u \leq 0$ ) is computed to take into account the effect of wave asymmetry. The new formula provides satisfactory agreement with the extensive data set that was compiled, and the best agreement compared to other formulas previously proposed.

The effect of the critical Shields parameter $\left(\theta_{c r}\right)$ was examined, and an exponential function of the ratio $\theta_{c r} / \theta_{c w}$ was proposed ( $\theta_{c w}$ is the maximum Shields parameter for the specific flow situation). This relationship significantly improves agreement with data for both steady current and oscillatory flow (wave) cases.

The net sediment transport by waves produced a transport coefficient that was smaller than expected. A coefficient value of $a=6$ was found (Equation 90), although it reached $a=12$ for a steady current (Equation 81). This value for the waves may be due to difficulties in estimating the bed roughness and to the influence of phase lag between fluid and sediment. Thus, it seems that phase-lag effects might be present even for small wave orbital velocities and coarser sediment, which introduce a weaker net sediment transport over a wave period.

Some discrepancy remains because the total shear stress is unknown for many of the experiments. For the experiments with oscillatory flows, the total shear stress must be estimated based on theoretical values. Two calculation approaches were presented, either using the Wilson (1966) formula to compute the Nikuradse roughness or using the Nikuradse skin 
roughness (even if it is known that the roughness increases strongly if sheet flow occurs). Depending on the data set, one or the other of these formulas gives the best agreement. This result emphasizes how important it is to accurately estimate the bottom shear stress if sheet flow occurs to predict the sediment transport rate accurately.

Because the bed-load formula does not take into account the effect of phase lag, adding a coefficient quantifying this effect should increase its accuracy. The phase-lag phenomenon is the main nonsteady effect due to oscillatory flows: a quantity of sand can still remain mobilized in the bed layer after each half-cycle of the wave velocity profile, and hence, move in the other direction. Dibajnia and Watanabe (1992) introduced a semiempirical formula that allows estimation of phase-lag effects. DohmenJ anssen (1999) and Camenen and Larroudé (2003) also proposed some semi-empirical coefficients to estimate the phase lag.

Regarding suspended load, a study of sediment concentration profiles using a large data set showed that an exponential profile overall gives a correct prediction of the profile shape. Assuming that the time-averaged current velocity is constant over the depth, the resulting sediment transport may be estimated from a simple equation. The two main parameters in the concentration profile are the mean sediment diffusivity over the depth and the bottom reference concentration. Comparison with measured velocity and concentration profiles over the depth showed that the assumption of a constant velocity over the depth does not significantly affect the results for most situations, especially for a steady current. If complex flows occur, such as undertow in the surf zone, the results are, in general, more scattered, but still good.

A prediction equation for the sediment diffusivity was proposed assuming a linear combination of the mixing generated by breaking waves and the mixing by energy dissipation in the bottom boundary layer due to the mean current and waves. For the dissipation in the boundary layer, the dissipation by the current/ waves was expressed as the product between a force (bottom shear stress) and a velocity (shear velocity) in order to be coherent with the classical mixing length approach (where $\varepsilon=\sigma \mathrm{\kappa} / 6 u * h$; Rouse 1938; Dally and Dean 1984). An estimation of the Schmidt number $\sigma$ was proposed for the current data and wave data separately. For the mixing due to breaking waves, an efficiency coefficient was introduced 
characterizing the energy dissipation due to breaking waves, and its value was determined through calibration against experimental data.

Following the approach by Madsen (1993), the reference concentration was found to be proportional to the mean Shields parameter including the effect of the critical Shields parameter. The results showed scatter mainly because of uncertainty in the prediction of the total Shields parameter including the effects of the bed forms. The formulas proposed by Van Rijn (1989) were adopted to estimate the ripple geometry and the Nikuradse roughness if no measurements were available.

Furthermore, as Van Rijn (1993) noted, the dimensionless grain size $d *$ should be taken into account in calculation of the reference concentration. It was introduced in the formula as a reduction factor for larger grain size. A study of sediment transport by breaking waves on a sloping beach showed that plunging breaking waves may increase the reference concentration. This effect was taken into account using an empirically derived formula based on the Irribaren parameter.

If ripples are present, a strong phase lag in the suspended load may be observed (Van der Werf and Ribberink 2004). One of the main parameters controlling this phase lag appears to be the ratio between the ripple height and the median grain size. A similar approach as for describing the phase lag in bed-load transport for the sheet-flow regime was proposed, which overall yielded good results.

The resulting formula for the suspended load appears to be robust and effective. It gives the best results among the studied formulas for most data sets. Also, because this formula is physically based, any improvement in knowledge concerning sediment transport processes (for example, estimation of the total shear stress) could be taken into account and thus is expected to improve its predictive capability. 


\section{References}

Abou-Seida, M. M. 1965. Bed load function due to wave action. Hydraulics Engineering Laboratory Technical Report HEL-2-11. Berkeley, CA: University of California.

Ahilan, R. V., and J . F. A. Sleath. 1987. Sediment transport in oscillatory flow over flat beds. Journal of Hydraulic Engineering 113(3):308-322.

Ahmed, A. S., and S. Sato. 2003. A sheet-flow transport model for asymmetric oscillatory flow: Part I: Uniform grain size sediments. Coastal Engineering Journal 45(3):321-337.

Al Salem, A. A. 1993. Sediment transport in oscillatory boundary layers under sheet flow conditions. PhD dissertation. The Netherlands: Delft Hydraulics.

Anderson, A. G. 1942. Distribution of suspended sediment in a natural stream. Transactions American Geophysical Union 23(2):678-683.

Antsyferov, S. M., T. Basinski, and N. V. Pykhov. 1983. Measurements of coastal suspended sediment concentrations. Coastal Engineering 7(2):145-166.

Antunes Do Carmo, J . S., A. Temperville, and F. J . Seabra-Santos. 2003. Bottom friction and time-dependent shear stress for wave-current interaction. Journal of Hydraulic Research 41(1):27-37.

Asano, T. 1992. Observations of granular-fluid mixture under an oscillatory sheet flow. Proceedings $23^{\text {rd }}$ Coastal Engineering Conference, ASCE, 1,896-1,909.

Bagnold, R. A. 1966. An approach to the sediment transport problem from general physics. Professional Paper 422-I. Washington, DC: U.S. Geological Survey.

Bailard, J . A. 1981. An energetic total load sediment transport model for a plane sloping beach. Journal of Geophysical Research 86(C11):10,938-10,954.

Bailard, J . A., and D. L. Inman. 1981. An energetics bed load model for a plane sloping beach: Local transport. Journal of Geophysical Research 86(C3):2,035-2,043.

Barton, J . R., and P. N. Lin. 1955. A study of the sediment transport in alluvial channels. Technical Report 55J RBZ. Fort Collins, CO: Civil Engineering Department, Colorado College, U.S.A.

Bayram, A., B. Camenen, and M. Larson. 2003. Equivalent roughness under sheet flow conditions. Proceedings Coastal Sediments 'o3, ASCE (CDROM).

Bayram, A., M. Larson, H. C. Miller, and N. C. Kraus. 2001. Cross-shore distribution of longshore sediment transport: Comparison between predictive formulas and field measurements. Coastal Engineering 44(2):79-99.

Bijker, E. W. 1967. Some considerations about scales for coastal models with movable bed. Technical Report 50. The Netherlands: Delft Hydraulics Laboratory. 
Bijker, E. W. 1968. Littoral drift as function of waves and current. Proceedings $11^{\text {th }}$ Coastal Engineering Conference, ASCE, 415-435.

Bosman, J . 1982. Concentration distribution under waves and current. Technical Report M1875. The Netherlands: Coastal Engineering Department, Delft University of Technology, (in Dutch).

Boussinesq, J . 1872. Theorie des ondes et des remous qui se propagent le long d'un canal rectangulaire horizontal. Journal Mathematique Pure et Applique 2(17):55-108.

Bowen, A. J . 1980. Simple models of nearshore sedimentation, beach profiles and longshore bars. In The Coastline of Canada, S. B. McCann (ed.), Geological Survey of Canada, Paper 80-10, 1-11.

Brownlie, W. R. 1981. Compilation of alluvial channel data: laboratory and field. Technical Report KH-R-43B. Pasadena, CA: California Institute of Technology, U.S.A.

Buttolph, A. M., C. W. Reed, N. C. Kraus, N. Ono, M. Larson, B. Camenen, H. Hanson, T. Wamsley, and A. K. Zundel. 2006. Two-dimensional depth-averaged circulation model CMS-M2D: Version 3.o, Report 2: Sediment transport and morphology change. Coastal and Hydraulics Laboratory Technical Report ERDC/CHL TR-069. Vicksburg, MS: U.S. Army Engineer Research and Development Center, U.S.A.

Camenen, B. 2002. Modelisation numerique du transport sedimentaire sur une plage sableuse. Unpublished PhD thesis. Grenoble, France: Universite J oseph Fourier. . 2007. Simple and general formula for the settling velocity of particles. Journal of Hydraulic Engineering 133(2): 229-233.

Camenen, B., A. Bayram, and M. Larson. 2006. Equivalent roughness height for plane bed under steady flow. Journal of Hydraulic Engineering 132(11):1,146-1,158.

Camenen, B., and P. Larroudé. 2003. Comparison of sediment transport formulae for a coastal environment. Coastal Engineering 48(2):111-132.

Camenen, B., and M. Larson. 2005a. Bed-load transport under steady and oscillatory flow. Proceedings Coastal Dynamics o5', ASCE (CDROM).

. 2005b. A general formula for non-cohesive bed load sediment transport.

Estuarine, Coastal, and Shelf Science 63(1-2):249-260.

. 2006. Phase lag effects in sheet flow transport. Coastal Engineering

53(5/6):531-542.

. 2007. A suspended load sediment transport formula for the nearshore. Journal of Coastal Research, in press.

Camenen, B., M. Larson, and T. Yamashita. 2005. A suspended load formula for the nearshore. Proceedings $52^{\text {nd }}$ Japanese Coastal Engineering Conference, J SCE, 381-385 (in J apanese). 
Chan, K. W., M. H. I. Baird, and G. F. Round. 1972. Behaviour of beds of dense particles in a horizontally oscillating liquid. Proceedings Royal Society of London $\mathrm{A}(330): 537-559$.

Cheng, N.-S. 2002. Exponential formula for bed load transport. Journal of Hydraulic Engineering 128(10):942-946.

Chung, D. H., B. T. Grasmeijer, and L. C. Van Rijn. 2000. Wave-related suspended sand transport in ripple regime. Proceedings $27^{\text {th }}$ Coastal Engineering Conference, ASCE, 2,836-2,849.

Clubb, G. 2001. Experimental study of vortex ripples in full-scale sinusoidal and asymmetric flows. Unpublished $\mathrm{PhD}$ dissertation. United Kingdom: Aberdeen University.

Coleman, N. L. 1970. Flume studies of the sediment transfer coefficient. Water Resources Research 6(3):801-809. . 1981. Velocity profiles with suspended sediment. Journal of Hydraulic Research 19(3):211-229.

Coles, D. 1956. The law of the wake in the turbulent boundary layer. Journal of Fluid Mechanics 1(Part 2):191-226.

Culbertson, J . K., C. H. Scott, and J. P. Bennet. 1972. Alluvial-channel data from Rio Grande conveyance channel, New Mexico, 1965-69. Technical Report 562-J, 49 pp. Washington, DC: U.S. Geological Survey, Professional Paper.

Dally, W. R., and R. G. Dean. 1984. Suspended sediment transport and beach profile evolution. Journal of Waterways, Port, Coastal and Ocean Engineering 110(1):15-33.

Damgaard, J . S., R. L. Soulsby, A. H. Peet, and S. Wright. 2003. Sand transport on steeply sloping plane and rippled beds. Journal of Hydraulic Engineering 129(9):706719 .

Davies, A. G. 1990. Modelling of the vertical distribution of suspended sediment in combined wave-current flow. Proceedings $5^{\text {th }}$ International Conference on the Physics of Estuaries and Coastal Seas. Gregynog: University of Wales.

Davies, A. G., J . S. Ribberink, A. Temperville, and J . A. Zyserman, J . 1997. Comparisons between sediment transport models and observations made in wave and current flows above plane beds. Coastal Engineering 31(1-4):163-198.

Davies, A. G., R. L. Soulsby, and H. L. King. 1988. A numerical model of the combined wave and current boundary layer. Journal of Geophysical Research 93(C1):491508.

Dette, H., and K. Uliczka. 1986. Velocity and sediment concentration fields across surf zones. Proceedings $20^{\text {th }}$ Coastal Engineering Conference, ASCE, 1,062-1,076.

Dibajnia, M. 1991. Study on nonlinear effects in beach processes. Unpublished $\mathrm{PhD}$ thesis. J apan: University of Tokyo. 
Dibajnia, M. 1995. Sheet flow transport formula extended and applied to horizontal plane problems. Coastal Engineering in Japan 38(2):178-194.

Dibajnia, M., and A. Watanabe. 1992. Sheet flow under nonlinear waves and currents. Proceedings $23^{\text {rd }}$ Coastal Engineering Conference, ASCE, 2,015-2,029.

Dibajnia, M., T. Moriya, and A. Watanabe. 2001. A representative wave model for estimation of nearshore local transport rate. Coastal Engineering Journal 43(1):1-38.

Dohmen-J anssen, C. M. 1999. Grain size influence on sediment transport in oscillatory sheet flow, phase-lags and mobile-bed effects. Unpublished $\mathrm{PhD}$ dissertation. The Netherlands: Delft University of Technology.

Dohmen-J anssen, C. M., and D. M. Hanes. 2002. Sheet flow dynamics under monochromatic nonbreaking waves. Journal of Geophysical Research 107(C10):13:1-13:21.

Dohmen-J anssen, C. M., D. Kroekenstoel, D. M. Hanes, and J . S. Ribberink. 2002. Phase lags in oscillatory sheet flow: Experiments and bed load modeling. Coastal Engineering 46(1):61-87.

Drake, T. G., and J . Calantoni. 2001. Discrete particle model for sheet flow sediment transport in the nearshore. Journal of Geophysical Research 106(C9):19,85919,868 .

DuToit, C. G., and J . F. A. Sleath. 1981. Velocity measurements close to rippled beds in oscillatory flow. Journal of Fluid Mechanics 112:71-96.

Einstein, H. A. 1950. The bed-load function for sediment transportation in open channel flows. Technical Report 1026. Washington, DC: U.S. Department of Agriculture, Technical Bulletin, U.S.A.

Engelund, F., and J . Fredsøe. 1976. A sediment transport model for straight channel. Nordic Hydrology 7(5):293-306.

Engelund, F., and E. Hansen. 1972. A Monograph on sediment transport in alluvial streams. Copenhagen: Technical Press Education.

Falques, A., and H. E. De Swart. 1998. Coastal morphodynamics. Technical Report, Institute of Marine, and Atmospheric Research, Utrecht, EG/ MASTY Advanced Study Course. The Netherlands: Renesse.

Fredsøe, J . 1984. Turbulent boundary layer in wave-current motion. Journal of Hydraulic Engineering 110(8):1,103-1,120.

Fredsøe, J ., O. H. Andersen, and S. Silberg. 1985. Distribution of suspended sediment in large waves. Journal of Waterways, Port, Coastal and Ocean Engineering 111(6):1,041-1,059.

Fredsøe, J ., and R. Deigaard. 1994. Mechanics of coastal sediment transport. Vol. 3, Advanced Series on Ocean Engineering, World Scientific Publication. 
Frijlink, H. C. 1952. Discussion des formules de debit solide de Kalinske, Einstein et Meyer-Peter and Müller compte tenue des mesures recentes de transport dans les rivieres Nederlandaises. $2^{\text {nd }}$ Journal Hydraulique, Societe Hydraulique de France, 98-103.

Gailani, J . Z., and J. S. Smith. 2000. Analysis of sediment transport processes, mouth of Columbia River. Coastal and Hydraulics Laboratory unpublished manuscript. Vicksburg, MS: U.S. Army Engineer Research and Development Center.

Gilbert, G. K. 1914. The transportation of debris by running water. Technical Report 86, U.S. Geological Survey, Professional Paper.

Grant, W. D., and O. S. Madsen. 1979. Combined wave and current interaction with a rough bottom. Journal of Geophysical Research 84(C4):1,797-1,808. . 1982. Movable bed roughness in unsteady oscillatory flow. Journal of Geophysical Research 87(C1):469-481.

Grasmeijer, B. 2002. Process-based cross-shore modelling of barred beaches. Unpublished $\mathrm{PhD}$ dissertation. The Netherlands: Utrecht University, Utrecht.

Green, M. O., T. J . Dolphin, A. Swales, and C. E. Vincent. 1999. Transport of mixed-size sediments in a tidal channel. Proceedings Coastal Sediments '99, ASCE, 644-658.

Hanson, H., and B. Camenen. 2007. Closed form solution for threshold velocity for initiation of sediment motion under waves. Proceedings Coastal Sediments 'O7, ASCE, 15-27.

Havinga, F. J . 1992. Sediment concentrations and sediment transport in case of irregular non-breaking waves with a current. Technical report, Coastal Engineering Department. The Netherlands: Delft University of Technology, Delft.

Hayakawa, N., G. Tsujimoto, and H. Hashimoto. 1983. Velocity distribution and suspended sediment concentration over large scale ripples. Coastal Engineering in Japan 26:91-100.

Homma, M., K. Horikawa, and R. Kajima. 1965. A study of suspended sediment due to wave action. Coastal Engineering in Japan 8:85-103.

Horikawa, K., A. Watanabe, and S. Katori. 1982. Sediment transport under sheet flow conditions. Proceedings $18^{\text {th }}$ Coastal Engineering Conference, ASCE, 1,3351,352 .

Huynh-Thanh, S., and A. Temperville. 1991. A numerical model of the rough turbulent boundary layer in combined wave and current interaction. In "Sand transport in rivers estuaries and the sea," Euromech 262:141-148.

J enkins, J. T., and D. M. Hanes. 1998. A sheared layer of colliding grains driven from above by a turbulent fluid. Journal of Fluid Mechanics 370:29-52.

J onsson. I. G. 1966. Wave boundary layers and friction factors. Proceedings $10^{\text {th }}$ Coastal Engineering Conference, ASCE, 127-148. 
Kajiura, K. 1968. A model of the bottom boundary layer in water waves. Bulletin of the Earthquake Research Institute 46:75-123.

Kalkanis, G. 1964. Transportation of bed material due to wave action. Technical Memorandum 2. Washington, DC: Coastal Engineering Research Center, U.S. Army Corps of Engineers.

Kim, H. 2004. Effective form roughness of ripples for waves. Journal of Coastal Research 20(3):731-738.

King, D. B. 1991. Studies in oscillatory flow bed load sediment transport. Unpublished $\mathrm{PhD}$ dissertation. San Diego, CA: University of California.

Komar, P. D., and M. C. Miller. 1975. The initiation of oscillatory ripple marks and the development of plane-bed at high shear stresses under waves. Journal of Sedimentary Petrology 45(3):697-703.

Kosyan, R. D. 1985. Vertical distribution of suspended sediment concentrations seawards of the breaking zone. Coastal Engineering 9(2):171-187.

Kraus, N. C., and M. Larson. 2001. Mathematical model for rapid estimation of infilling and sand bypassing at inlet entrance channels. Coastal and Hydraulics Laboratory Technical Note CHETN-IV-35. Vicksburg, MS: U.S. Army Engineer Research and Development Center, USA.

Kroon, A. 1991. Suspended-sediment concentrations in a barred nearshore zone. Proceedings Coastal Sediments '91, ASCE, 328-341.

Larson, M. 1995. Model for decay of random waves in surf zone. Journal of Waterways, Port, Coastal and Ocean Engineering 121(1):1-12.

Laursen, E. M. 1958. The total sediment load of streams. Journal of Hydraulic Engineering 84(1):1-36.

Lees, B. J . 1981. Relationship between eddy viscosity of sea water and eddy diffusivity of suspended sediments. Geo-Marine Letters 1:249-254.

Li, L., and M. Sawamoto. 1995. Experiments on sediment transport in sheet-flow regime under oscillatory flow. Coastal Engineering in Japan 38(2):157-178.

Madsen, O. S. 1991. Mechanics of cohesionless sediment transport in coastal waters. Proceedings Coastal Sediments '91, ASCE, 15-27.

. 1993. Sediment transport outside the surf zone. Coastal Engineering Research Center, unpublished report. Vicksburg, MS: U.S. Army Engineer Waterways Experiment Station, USA.

Madsen, O. S., and W. D. Grant. 1976. Sediment transport in the coastal environment. Technical Report 209. Cambridge, MA: Massachusetts Institute of Technology, USA. 
Madsen, O. S., Y. Tajima, and B. A. Ebersole. 2003. Longshore sediment transport: a realistic order-of-magnitude estimate. Proceedings Coastal Sediments 'o3, ASCE (CDROM).

Manohar, M. 1955. Mechanics of bottom sediment movement due to wave action. Beach Erosion Board Technical Memorandum 75. Washington, DC: U.S. Army Corps of Engineers.

Meyer-Peter, E., and R. Müller. 1948. Formulas for bed-load transport. $2^{\text {nd }}$ Meeting of the International Association of Hydraulic Structures Research, 39-64. Stockholm, Sweden.

Migniot, C. 1977. Action des courants, de la houleet duvent sur les sediments. La Houille Blanche 1:9-47.

Miller, H. C. 1998. Comparison of storm longshore transport rates to predictions. Proceedings $26^{\text {th }}$ International Coastal Engineering Conference, ASCE, 2,9542,967 . . 1999. Field measurements of longshore sediment transport during storm. Coastal Engineering 36(4):301-321.

Nakato, T., F. A. Locher, J. R. Glover, and J . F. Kennedy. 1977. Wave entrainment of sediment from rippled beds. Journal of Waterways, Port, Coastal and Ocean Engineering 103:83-100.

Nielsen, P. 1979. Some basic concepts of wave sediment transport. Technical report, 160 pp. Denmark: Institute of Hydrodynamics and Hydraulic Engineering (ISVA), Technical University of Denmark. . 1981. Dynamics and geometry of wave-generated ripples. Jurnal of Geophysical Research 86(C7):6,467-6,472.

. 1984. Field measurements of time-averaged suspended sediment concentrations under waves. Coastal Engineering 8(1):51-72.

. 1986. Suspended sediment concentrations under waves. Coastal Engineering 10(1):23-31.

. 1992. Coastal bottom boundary layers and sediment transport. Vol. 4 of Advanced Series on Ocean Engineering, World Scientific Publication.

Nikora, V. I, and G. M. Smart. 1997. Turbulence characteristics of New Zealand gravelbed rivers. Journal of Hydraulic Engineering 123(9):764-773.

Nnadi, F. N., and K. C. Wilson. 1992. Motion of contact-load particles at high shear stress. Journal of Hydraulic Engineering 118(12):1,670-1,684.

Ogston, A. S., and R. W. Sternberg. 2002. Effect of wave breaking on sediment eddy diffusivity, suspended-sediment, and longshore sediment flux profiles in the surf zone. Continental Shelf Research 22(4):633-655. 
Peters, K. 2000. Morphodynamics in the surf zone of sandy coasts. Unpublished PhD dissertation. Germany: Technical University of Braunschweig (in German).

Rattanapitikon, W., and T. Shibayama. 2000. Simple model for undertow profile. Coastal Engineering Journal 42(1):1-30.

Raudkivi, A. J . 1998. Loose boundary hydraulics. Balkema. The Netherlands: Rotterdam.

Ribberink, J . S. 1998. Bed-load transport for steady flows and unsteady oscillatory flows. Coastal Engineering 34(1):59-82.

Ribberink, J. S., and A. A. Al Salem. 1994. Sediment transport in oscillatory boundary layers in cases of rippled beds and sheet flow. Journal of Geophysical Research 99(C6):707-727.

Ribberink, J. S., and Z. Chen. 1993. Sediment transport of fine sand under asymmetric oscillatory flow. Report H840 (Part VII). The Netherlands: Delft Hydraulics.

Richardson, J. F., and W. N. Zaki. 1954. Sedimentation and fluidization: Part I. Transactions Institution of Chemical Engineers 32:35-53.

Rose, C. P., and P. D. Thorne. 2001. Measurements of suspended sediment transport parameters in a tidal estuary. Continental Shelf Research 21(15):1,551-1,575.

Rouse, H. 1938. Experiments on the mechanics of sediment suspension. Proceedings $5^{\text {th }}$ International Congress for Applied Mechanics Proceedings 55. New York: Wiley and Sons.

Rowe, P. N. 1987. A convenient empirical equation for estimation of the Richardson-Zaki exponent. Chemical Engineering Science 42:2,795-2,796.

Sato, S. 1987. Oscillatory boundary layer flow and sand movement over ripples. Unpublished PhD dissertation. Tokyo, J apan: University of Tokyo.

Sawamoto, M., and T. Yamashita. 1986. Sediment transport rate due to wave action. Journal of Hydroscience and Hydraulic Engineering 4(1):1-15.

Scott, G. H., and H. D. Stephens. 1966. Special sediment investigation, Mississippi River at St Louis, Missouri, 1961-1963. Technical Report 1819-J . Washington, DC: Geological Survey Water-Supply Paper.

Shibayama, T., and K. Horikawa. 1982. Sediment transport and beach transformation. Proceedings $18^{\text {th }}$ Coastal Engineering Conference, ASCE, 1,509-1,522.

Shields, A. 1936. Anwendung der Ähnlichkeitsmechanik und der Turbulenzforschung auf die Geshiebebewegung. Preussische Versuchsanstalt fur Wasserbau und Schiffbau. Heft 26, 5-24.

Skafel, M. G., and B. G. Krishnappan. 1984. Suspended sediment distribution in wave field. Journal of Waterways, Port, Coastal and Ocean Engineering 110(2):215230. 
Sleath, J . F. A. 1978. Measurements of bedload in oscillatory glow. Journal of Waterways, Port, Coastal and Ocean Engineering 10(4):291-307.

. 1982. The suspension of sand by waves. Journal of Hydraulic Research 20(5):439-451.

Smart, G. M. 1984. Sediment transport formula for steep channels. Journal of Hydraulic Engineering 111(3):267-276.

Smart, G. M. 1999. Turbulent velocity profiles and boundary shear in gravel bed rivers. Journal of Hydraulic Engineering 125(2):106-116.

Smith, J . D., and McLean, S. R. 1977. Spatially averaged flow over a wavy surface. Journal of Geophysical Research 82(12):1,735-1,746.

Soulsby, R. L. 1997. Dynamics of marine sands, a manual for practical applications. H. R. Wallingford, UK: Thomas Telford.

Soulsby, R. L., L. Hamm, G. Klopman, D. Myrhaug, R. R. Simons, and G. P. Thomas. 1993. Wave-current interaction within and outside the boundary layer. Coastal Engineering 21(1-3):41-69.

Soulsby, R. L. and R. J . S. Whitehouse 2005a. Prediction of ripple properties in shelf seas. Mark 1 Predictor. HR Wallingford Report TR150, February. Wallingford, UK.

Soulsby, R. L. and R. J . S. Whitehouse 2005b. Prediction of ripple properties in shelf seas. Mark 2 Predictor for time evolution. HR Wallingford Report TR154, December. Wallingford, UK.

Soulsby, R. L., and R. S. Whitehouse. 1997. Threshold of sediment motion in coastal environment. Proceedings Conference on Pacific Coasts and Ports, 149-154. Christchurch, NewZealand: University of Canterbury.

Staub, C., I. G. J onsson, and I. A. Svendsen. 1984. Variation of sediment suspension in oscillatory flow. Proceedings $19^{\text {th }}$ Coastal Engineering Conference, ASCE, 2,3102,321 .

Steetzel, H. J. 1984. Sediment suspension in an oscillating water motion close to the sand bed. Technical report, Coastal Engineering Department. Delft, The Netherlands: Delft University of Technology, Delft (in Dutch).

. 1985. Model tests of scour near the toe of dune revetments. Technical Report M2051-Il, Coastal Engineering Department. Delft, The Netherlands: Delft University of Technology, Delft (in Dutch).

Stive, M. J . F. 1984. Energy dissipation in waves breaking on gentle beaches. Coastal Engineering 8(2):99-127.

Stokes, G. G. 1851. On the effect of internal friction of fluids on the motion of pendulums. Transactions of Cambridge Philosophical Society IX:8-106. 
Sumer, B. M., and R. Deigaard. 1981. Particle motions near the bottom in turbulent flow in an open channel (Part 2). Journal of Fluid Mechanics 109:311-337.

Svendsen, I. A. 1984. Mass flux and undertow in the surf zone. Coastal Engineering 8(4):347-365.

Svendsen, I. A., and J . B. Hansen. 1988. Cross-shore currents in surf-zone modeling. Coastal Engineering 12:23-42.

Swart, D. H. 1974. Offshore sediment transport and equilibrium beach profiles. Technical report. Delft, The Netherlands: Delft Hydraulics Laboratory.

Trowbridge, J., and O. S. Madsen. 1984a. Turbulent wave boundary layers, 1: Model formulation and first-order solution. Journal of Geophysical Research 89(C5):7,989-7,997.

. 1984b. Turbulent wave boundary layers, 2: Second-order theory and mass transport. Journal of Geophysical Research 89(C5):7,999-8,007.

Van der Velden, E. 1986. Sediment suspension in an oscillating water motion close to the sand bed. Technical report, Coastal Engineering Department. Delft, The Netherlands: Delft University of Technology (in Dutch).

Van der Werf, J . J ., and J . S. Ribberink. 2004. Wave-induced sediment transport processes in the ripple regime. Proceedings $29^{\text {th }}$ Coastal Engineering Conference, ASCE, 1,741-1,753.

Van Rijn, L. C. 1984a. Sediment transport: Part I: Bed load transport. Journal of Hydraulic Engineering 110(10):1,431-1,456. . 1984b. Sediment transport: Part II: Suspended load transport. Journal of Hydraulic Engineering 110(11):1,613-1,641.

. 1984c. Sediment transport: Part III: Bed form sand alluvial roughness. Journal of Hydraulic Engineering 110(12):1,733-1,754.

. 1989. Handbook of sediment transport by currents and waves. Technical Report H461. The Hague: Delft Hydraulics Laboratory.

. 1993. Principles of sediment transport in rivers, estuaries and coastal seas.

The Netherlands: Aqua Publications.

Van Rijn, L. C., A. G. Davies, J . Van der Graff, and J . S. Ribberink (eds.). 2001. SEDMOC: Sediment transport modelling in marine coastal environments. The Netherlands: Aqua Publications, Amsterdam.

Van Rijn, L. C., and G. L. Tan. 1985. Sutrench-model: Two-dimensional vertical mathematical model for sedimentation in dredges channels and trenches by currents and waves. Technical report. The Hague: Delft Hydraulics Laboratory.

Vanoni, V. A. 1946. Transportation of suspended sediment by water. Transactions American Society of Civil Engineers III:67-133. 
Voogt, L., L. C. Van Rijn, and J. H. Van den Berg. 1991. Sediment transport of fine sand at high velocities. Journal of Hydraulic Engineering 117(7):869-890.

Voulgaris, G., and M. B. Collins, M. 2000. Sediment resuspension on beaches: Response to breaking waves. Marine Geology 167(1-2):167-187.

Wang, P., B. A. Ebersole, and E. R. Smith. 2002. Longshore sand transport - initial results from large-scale sediment transport facility. Coastal and Hydraulics Laboratory Technical Note CHETN-II-46. Vicksburg, Mississippi: U.S. Army Engineer Research and Development Center, USA.

Watanabe, A. 1982. Numerical models of nearshore currents and beach deformation. Coastal Engineering in Japan 25:147-161.

Watanabe, A. 1989. Numerical models of nearshore currents and beach deformation. Coastal Engineering 12(4):371-379.

Watanabe, A., and M. Isobe. 1990. Sand transport rate under wave-current action. Proceedings 22 ${ }^{\text {nd }}$ Coastal Engineering Conference, ASCE, 2,495-2,506. . 1992. Total rate and distribution of longshore sand transport. Proceedings $23^{\text {rd }}$ Coastal Engineering, ASCE, 2,528-2,541.

Whitehouse, R. S. 1995. Observations of the boundary layer characteristics and the suspension of sand at a tidal site. Continental Shelf Research 15(13):1,549-1,567.

Willis, G. P., N. L. Coleman, and W. M. Ellis. 1972. Laboratory study of transport of fine sand. Journal of Hydraulic Engineering 98(3):801-816.

Wilson, K. C. 1966. Bed-load transport at high shear stress. Journal of Hydraulic Engineering 92(11):49-59. . 1989a. Friction of wave induced sheet flow. Coastal Engineering 12(4):371-379. . 1989b. Mobile bed friction at high shear stress. Journal of Hydraulic Engineering 115(6):825-830.

Yalin, M. S. 1977. Mechanics of Sediment Transport. Pergamon Press, Oxford, 2nd edition.

You, Z.-J . 1999. The inception of sheet flow in oscillatory sheet flow. Technical Note, Ocean Engineering 26(3):277-285.

Yu, Y., R.W. Sternberg, and R. A. Beach. 1993. Kinematics of breaking wave and associated suspended sediment in the nearshore zone. Continental Shelf Research 13(11):1,219-1,242.

Zala Flores, N., and J. F. A. Sleath. 1998. Mobile layer in oscillatory sheet flow. Journal of Geophysical Research 106(C6):12,783-12,793.

Zyserman, J . A., and J . Fredsøe. 1994. Data analysis is of bed concentration of suspended sediment. Journal of Hydraulic Research 120(9):1,021-1,042. 


\section{Appendix A: Notation}

\section{Roman letters}

$a_{w}, a_{n}, b$

$A, B$

$A_{c R}$

$A_{d w}, B_{d w}$

$A_{E}$

$A_{r}$

$A_{w}=U_{w} T_{w} /(2 \pi)$

$A_{\varepsilon}$

$c$

$c_{a}$

$c_{b}$

$c_{m}$

$c_{o}$

$c_{R}$

$c_{1}, c_{2}, c_{3}$

$C_{b}$

$C_{g}$

$C_{D}$

$C_{f}$

$C_{R b}$

$d$

$d_{k}$

$d_{50}$

$d_{*}=\left[(s-1) g / v^{2}\right]^{1 / 3} d_{50}$

$D$

$D_{b}$

$D_{c}$

$D_{p l}$ coefficients for the Camenen and Larson formula (2005b)

coefficients used in various equations

empirical coefficient in the reference concentration formula

coefficients for the Dibajnia and Watanabe formula (1992)

power in the Einstein integrals

coefficient for ripple roughness calculation

orbital amplitude (semi-excursion) of wave motion at the bed

wave breaking dissipation coefficient

volume concentration

reference volume concentration at the level $z_{a}$

concentration of particles at the top of the moving mixing layer

maximum volume concentration

concentration of particles inside the fixed bed

reference volume concentration

coefficient values in shear stress relationship developed by Engelund and Hansen (1972)

breaking wave parameter from Bijker (1968)

group speed of waves

drag coefficient applicable to depth-averaged current

drag coefficient

multiplicative coefficient for the influence of breaker type on the reference concentration

grain diameter

grain diameter for which $k \%$ of the grains by mass is finer

median grain size

dimensionless grain size

total effective energy dissipation

energy dissipation due to wave breaking

energy dissipation from bottom friction due to the current

coefficient in correction factor $\alpha_{p l, s}$ for phase lag in suspended load 


\begin{tabular}{|c|c|}
\hline$D_{w}$ & energy dissipation from bottom friction due to the waves \\
\hline$\vec{e}_{z}$ & vertical unit vector \\
\hline$E_{r m s}$ & root-mean-square error \\
\hline$E_{w}$ & wave energy \\
\hline$f_{c}$ & current-related friction coefficient \\
\hline$f_{c w}$ & friction coefficient for wave and current interaction \\
\hline$f_{w}$ & wave-related friction coefficient \\
\hline$F$ & total sediment flux \\
\hline$F_{a}$ & sediment flux due to the advection \\
\hline$F_{d}$ & sediment flux due to the diffusion \\
\hline$F_{r}$ & Froude number \\
\hline$F_{s}$ & sediment flux due to the settling \\
\hline$F_{w}=E_{w} C_{g}$ & wave energy flux \\
\hline$g=9.81 \mathrm{~ms}^{-1}$ & acceleration due to gravity \\
\hline$h$ & water depth \\
\hline$h_{t}$ & Water depth at the wave trough level \\
\hline$H_{d}$ & height of dunes \\
\hline$H_{m o}$ & energy-based significant wave height \\
\hline$H_{r c}$ & height of current ripples \\
\hline$H_{r w}$ & height of wave ripples \\
\hline$H_{s}$ & significant wave height \\
\hline$H_{w}$ & wave height \\
\hline$H_{r m s}$ & root-mean-square wave height \\
\hline$I_{1}, I_{2}$ & Einstein integrals for suspended load \\
\hline$k_{a}$ & apparent roughness height \\
\hline$k_{w}=2 \pi / L_{w}$ & wave number \\
\hline$k_{s}$ & roughness height \\
\hline$k_{s, d}$ & roughness height due to dunes \\
\hline$k_{s, g}$ & roughness height due to skin friction \\
\hline$k_{s, r}$ & roughness height due to ripples \\
\hline$k_{s, s f}$ & roughness height due to sheet flow \\
\hline K & Strickler parameter \\
\hline$K_{1}, K_{2}$ & coefficients \\
\hline
\end{tabular}




\begin{tabular}{|c|c|}
\hline$L_{d}$ & length of dunes \\
\hline$L_{r c}$ & length of current ripples \\
\hline$L_{r w}$ & length of wave ripples \\
\hline$L_{w}$ & wavelength \\
\hline$m$ & mean slope of the beach \\
\hline$m_{0}, m_{2}$ & zero'th and second moment of the wave spectrum \\
\hline $\operatorname{Mean}(f)$ & mean value of the function $f$ \\
\hline$n$ & empirical power used in various equations \\
\hline$\vec{n}$ & unit vector perpendicular to a surface \\
\hline$p$ & porosity \\
\hline$p_{p l}$ & phase lag parameter (Dohmen-Janssen 1999) \\
\hline$P_{k}$ & prediction within a factor $k$ (in \%) \\
\hline$P_{R}=W_{s} /\left(\kappa u_{*}\right)$ & Rouse parameter \\
\hline$P_{W R}=H_{r} / d_{50}$ & vortex suspension parameter \\
\hline$q_{s}$ & volumetric sediment transport rate \\
\hline$q_{s b}$ & volumetric bed-load sediment transport rate \\
\hline$q_{s s}$ & volumetric suspended load sediment transport rate \\
\hline$r=A_{w} / k_{s}$ & relative roughness \\
\hline$r_{p l}$ & sediment transport reduction due to phase-lag effects \\
\hline$r_{p l, C L}$ & $\begin{array}{l}\text { sediment transport reduction due to phase-lag effects according to } \\
\text { Camenen and Larson (2006) }\end{array}$ \\
\hline$r_{p l, D W}$ & $\begin{array}{l}\text { sediment transport reduction due to phase-lag effects according to } \\
\text { Dibajnia and Watanabe (1992) }\end{array}$ \\
\hline$r_{p l, s}$ & $\begin{array}{l}\text { sediment transport reduction due to phase-lag effects for } \\
\text { suspended load }\end{array}$ \\
\hline$r_{u}=U_{c} / U_{w}$ & dimensionless steady velocity \\
\hline$r_{w}=u_{w, \max } / U_{w}-1$ & wave asymmetry coefficient \\
\hline$s=\rho_{s} / \rho$ & relative density of particle \\
\hline$S$ & erosion/deposition flux due to the suspended load \\
\hline $\operatorname{Std}(f)$ & standard deviation of the function $f$ \\
\hline$S_{*}=\sqrt{(s-1) g d_{50}{ }^{3}} /(4 v)$ & dimensionless immersed sediment weight \\
\hline$t$ & time \\
\hline$T$ & flow period \\
\hline$T_{m}$ & mean period of irregular waves \\
\hline$T_{p}$ & peak period of irregular waves (peak of the wave spectrum) \\
\hline
\end{tabular}




\begin{tabular}{|c|c|}
\hline$T_{w}$ & wave period \\
\hline$T_{w c}, T_{w t}$ & half-wave periods where $u(t) \geq 0$ and $u(t)<0$, respectively \\
\hline$T_{\tau}=\left(\theta-\theta_{c r}\right) / \theta_{c r}$ & dimensionless bed shear stress parameter (Van Rijn 1984a) \\
\hline$u$ & horizontal velocity \\
\hline$u_{h}$ & horizontal velocity at water surface \\
\hline$u_{w}$ & wave orbital velocity \\
\hline$u_{w, \min }, \quad u_{w, \max }$ & minimum and maximum wave orbital velocity \\
\hline$u_{w c}, u_{w t}$ & $\begin{array}{l}\text { quadratic velocities (wave and current combined) over each half- } \\
\text { period (Dibajnia and Watanabe 1992) }\end{array}$ \\
\hline$u_{*}=\sqrt{\tau / \rho}$ & friction velocity \\
\hline$U_{c}$ & depth-averaged current velocity \\
\hline$U_{s}$ & speed of the bed-load layer \\
\hline$U_{w}$ & wave orbital velocity amplitude at seabed \\
\hline$V$ & volume \\
\hline$V_{c}, V_{t}$ & $\begin{array}{l}\text { characteristic velocity during onshore and offshore motion, } \\
\text { respectively }\end{array}$ \\
\hline$V_{\text {mat }}, V_{\text {tot }}$ & volume of matter and total volume, respectively \\
\hline$w$ & vertical velocity \\
\hline$W_{s}$ & settling velocity \\
\hline$W_{\text {sh }}$ & hindered settling velocity \\
\hline$W_{*}=W_{s} / \sqrt{(s-1) g d_{50}}$ & dimensionless settling velocity \\
\hline$x, y$ & horizontal coordinates \\
\hline$X=\cos \phi_{s}$ & parameter in formula for phase-log effects of sheet flow \\
\hline$X_{v}=\left|U_{c}\right| /\left(\left|U_{c}\right|+U_{w}\right)$ & linear function for wave and current interaction (velocity) \\
\hline$X_{p}=\cos \phi_{p l}$ & parameter in formula for phase-lag effects on suspended load \\
\hline$X_{p l, s}$ & $\begin{array}{l}\text { coefficient to take into account wave-related transport in the } \\
\text { suspended load }\end{array}$ \\
\hline$Y=\theta_{c} /\left(\theta_{c}+\theta_{w}\right)$ & linear function the wave and current interaction (shear stress) \\
\hline$z$ & vertical coordinate \\
\hline$z_{a}$ & $\begin{array}{l}\text { reference height (above the bed-load layer) at which reference } \\
\text { concentration } c_{a} \text { is estimated }\end{array}$ \\
\hline$z_{0}$ & bed roughness length \\
\hline$z_{w}$ & $\begin{array}{l}\text { elevation from the bed to where the sediment diffusivity is constar } \\
\text { (Van Rijn 1989) }\end{array}$ \\
\hline
\end{tabular}




\section{Greek letters}

\begin{tabular}{|c|c|}
\hline$\alpha_{B}, \alpha_{E}$ & $\begin{array}{l}\text { observed slope of concentration profile for a power law and an } \\
\text { exponential law, respectively }\end{array}$ \\
\hline$\alpha_{c w}$ & coefficient for the wave and current interaction (Van Rijn 1984a) \\
\hline$\alpha_{p l}$ & $\begin{array}{l}\text { correction factor for phase lag in the formula by Camenen and } \\
\text { Larson (2006) }\end{array}$ \\
\hline$\alpha_{p l, s}$ & correction factor for phase lag due suspended load \\
\hline$a_{1}, a_{2}, a_{3}$ & $\begin{array}{l}\text { coefficients in governing equation for water velocity profile under } \\
\text { varying bottom slope }\end{array}$ \\
\hline$\beta_{w}$ & dimensionless diffusion coefficient (Skafel and Krishnappan 1984) \\
\hline$\gamma_{b}=H_{w b} / h_{b}$ & breaker depth ratio \\
\hline$\Gamma$ & sediment availability (Dibajnia and Watanabe 1992) \\
\hline$\delta$ & boundary layer thickness \\
\hline$\delta_{b}$ & bed-load layer thickness \\
\hline$\delta_{m}$ & moving bed layer thickness \\
\hline$\delta_{s}$ & sheet-flow layer thickness \\
\hline$\delta_{w}=v T_{w} / \pi$ & wave-boundary layer thickness \\
\hline$\varepsilon$ & sediment diffusivity \\
\hline$\varepsilon_{b}, \varepsilon_{s}$ & bed load and suspended load efficiency (Bailard 1981) \\
\hline$\varepsilon_{h}, \varepsilon_{v}$ & horizontal and vertical sediment diffusivity \\
\hline$\theta$ & Shields parameter \\
\hline$\theta^{\prime}$ & skin Shields parameter \\
\hline$\theta_{c}$ & current-related Shields parameter \\
\hline$\theta_{c w}(t)$ & $\begin{array}{l}\text { instantaneous Shields parameter } \theta_{c w}(t) \text { for wave and current } \\
\text { interaction }\end{array}$ \\
\hline$\theta_{c w, m}$ & mean Shields parameter for wave and current interaction \\
\hline$\theta_{c w, \max }$ & maximum Shields parameter due to waves and current combined \\
\hline$\theta_{c w, n e t}$ & $\begin{array}{l}\text { net Shields parameter for wave and current interaction (Camenen } \\
\text { and Larson, 2005b) }\end{array}$ \\
\hline$\theta_{c w, \text { onshore }}$ & $\begin{array}{l}\text { mean value of the instantaneous Shields parameter } \theta_{c w}(t) \text { in the } \\
\text { first half-period where } \theta_{c w}(t) \geq 0 \text { (Camenen and Larson 2005b) }\end{array}$ \\
\hline$\theta_{c w, o f f s h o r e}$ & $\begin{array}{l}\text { mean value of the instantaneous Shields parameter } \theta_{c w}(t) \text { in the } \\
\text { second half-period where } \theta_{c w}(t)<0 \text { (Camenen and Larson, } \\
\text { 2005b) }\end{array}$ \\
\hline$\theta_{c r}$ & threshold Shields parameter for the inception of movement \\
\hline$\theta_{c r, s}$ & threshold Shields parameter for the inception of suspended load \\
\hline
\end{tabular}


$\theta$

$\theta_{M}$

$\theta_{r}$

$\theta_{T}$

$\theta_{w}$

$\theta_{w, m}$

$\kappa=0.41$

$\lambda_{w}=H_{w} / L_{w}$

$\mu$

$\mu_{c}=\left(f_{c t} / f_{c}\right)^{3 / 2}$

$\mu_{f}=f_{c} / f_{c t}$

$v=\mu / \rho$

$v_{i}=U_{c w} / W_{s}$

$v_{t}$

$\xi_{b}=m / \sqrt{\lambda_{w b}}$

$\xi_{\infty}=m / \sqrt{\lambda_{w \infty}}$

$\xi_{B}=\sqrt{f_{w t} / f_{c t}}$

$\bar{\omega}_{s}=W_{s} / u_{*}$

$\rho$

$\rho_{s}$

$\sigma_{B}$

$\sigma_{E}$

$\sigma_{P}$

$\sigma=\varepsilon_{v} / v_{v}$

$\sigma_{s}=\sqrt{d_{84} / d_{16}}$

$\tau$

$\tau_{b}$

$\phi$

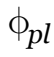

$\phi_{d}$ threshold Shields parameter for the inception of sheet flow

maximum Shields parameter

enhanced skin-friction Shields parameter due to ripples

transport-dependent Shields parameter

wave-related Shields parameter

mean wave-related Shields parameter

Von Karman's constant

wave steepness

dynamic viscosity

ripple parameter (Bijker 1968)

shape factor (Van Rijn 1984a)

kinematic viscosity

dimensionless parameter appearing in formula for $D_{p l}$

turbulent eddy viscosity

Irribaren parameter defined at the break point

Irribaren parameter defined offshore (deep water)

parameter for wave and current interaction (Bijker 1968)

suspension parameter

density of water

density of sediment grains

Schmidt number based on a power-law concentration profile (sediment diffusivity following a parabolic shape)

Schmidt number based on a exponential concentration profile

Schmidt number based on a power-law concentration profile (sediment diffusivity following a linear shape)

Schmidt number (ratio vertical eddy diffusivity of particles to vertical eddy viscosity)

geometric standard deviation of grain size

horizontal shear stress

bottom shear stress

internal friction angle of the sediments

phase lag between time-dependent sediment concentration profile and velocity profile in suspended load

phi-size of grains 


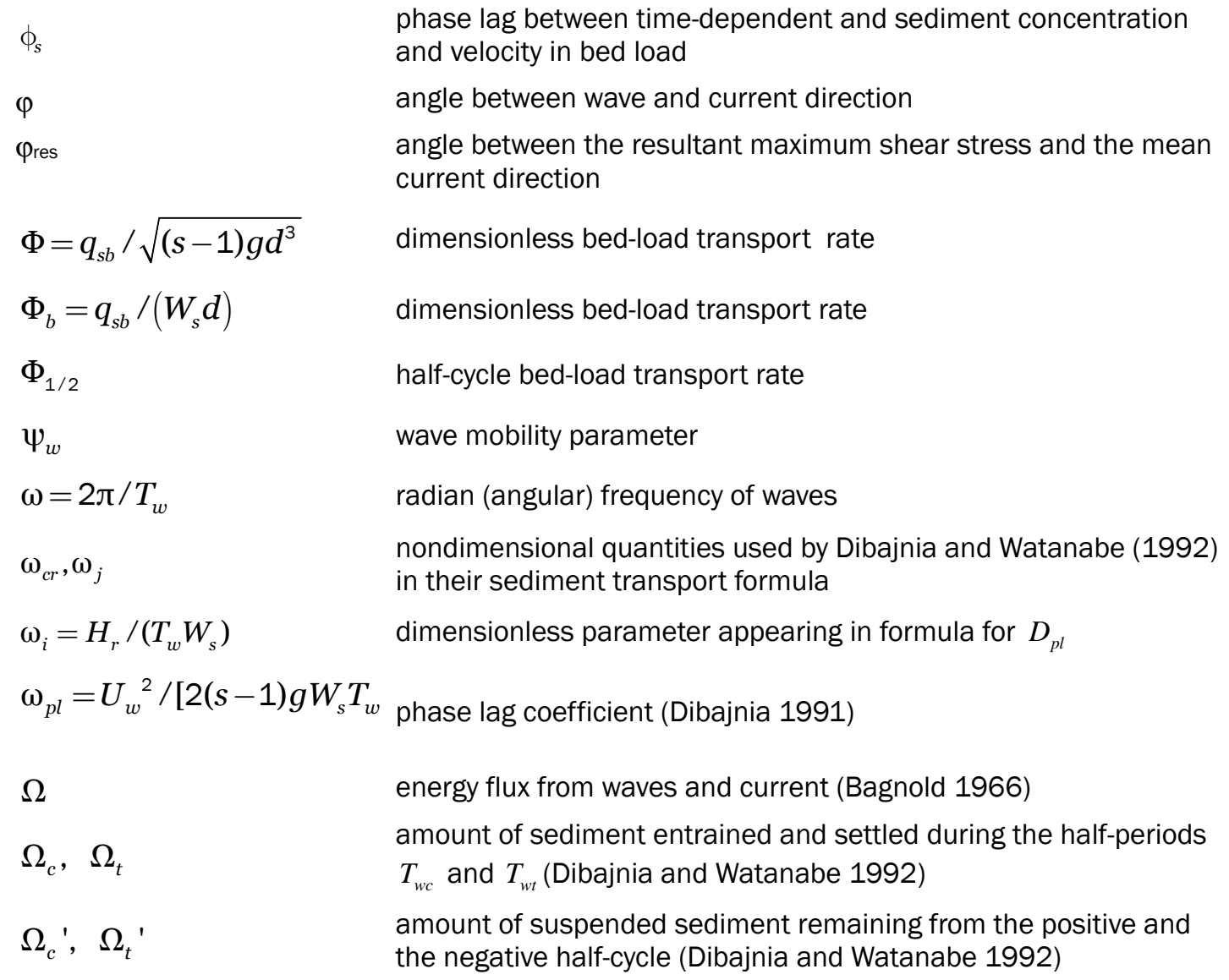

phase lag between time-dependent and sediment concentration and velocity in bed load

angle between wave and current direction

angle between the resultant maximum shear stress and the mean current direction

half-cycle bed-load transport rate

wave mobility parameter

radian (angular) frequency of waves

nondimensional quantities used by Dibajnia and Watanabe (1992)

in their sediment transport formula

dimensionless parameter appearing in formula for $D_{p l}$

phase lag coefficient (Dibajnia 1991)

energy flux from waves and current (Bagnold 1966)

amount of sediment entrained and settled during the half-periods $T_{w c}$ and $T_{w t}$ (Dibajnia and Watanabe 1992)

amount of suspended sediment remaining from the positive and the negative half-cycle (Dibajnia and Watanabe 1992)

\section{Other symbols}

$$
\begin{aligned}
& \Re_{*}=W_{s} d / v \\
& \Re_{*_{w}}=U_{w} d / v \\
& \Re_{w}=U_{w} A_{w} / v
\end{aligned}
$$

particle Reynolds number

wave-related particle Reynolds number

wave-related Reynolds number

\section{Subscripts}

$b$

bed

$c$

cr

$E, P, B$

$h$

$m$ break point value / pertaining to bed load

pertaining to the bed

pertaining to current

critical value

pertaining to an exponential, power-law, and Rouse concentration profile, respectively

horizontal value

mean value 


$\begin{array}{ll}\begin{array}{ll}\text { max } \\ \text { meas }\end{array} & \text { maximum value } \\ \text { min } & \text { measured value } \\ n & \text { minimum value } \\ n e t & \text { pertaining to the direction normal to the waves } \\ o & \text { net value } \\ \text { onshore } & \text { pertaining to offshore value (deep water) } \\ \text { offshore } & \text { pertaining to the direction of wave propagation } \\ \text { res } & \text { pertaining to the opposite direction of wave propagation } \\ \text { pred } & \text { residual value } \\ r m s & \text { predicted value } \\ s & \text { root-mean-square value } \\ s f & \text { pertaining to sediment / suspension } \\ t & \text { pertaining to sheet flow regime } \\ v & \text { total value } \\ w & \text { vertical value }\end{array}$

\section{General operators}

$\begin{array}{ll}\bar{X} & \text { time-averaged, steady component (of the variable } x \text { ) } \\ \tilde{X} & \text { periodic component (of the variable } x \text { ) } \\ \vec{X} & \text { vector (variable } x \text { ) } \\ <\quad>\quad & \text { time average }\end{array}$

\section{Abbreviations}

$\begin{array}{ll}\text { LSTF } & \text { Large Sediment Transport Facility } \\ \text { LWF } & \text { Large Wave Flume } \\ \text { OT } & \text { Oscillating Tray } \\ \text { OWT } & \text { Oscillating Water Tunnels }\end{array}$




\section{Appendix B: Computation of Mean Values for Onshore and Offshore Shields Parameter}

In this appendix, analytical solutions presented for the mean values of the onshore and offshore Shields parameter for various combinations of waves and a mean current, where either linear or $2^{\text {nd }}$-order Stokes wave theory is employed. Linear wave theory yields a simple sinusoidal variation in the velocity with time, whereas the $2^{\text {nd }}$-order Stokes theory produces an additional higher-order term that varies at twice the frequency compared to the basic sinusoidal wave.

\section{Sinusoidal wave without current}

For a sinusoidal wave (i.e., $u_{w}(t)=U_{w} \cos \omega t$, where $\omega=2 \pi / T_{w}$ and $U_{w}$ is the wave orbital velocity at the bottom, and $T_{w}$ the wave period), the following relationship is obtained:

$$
\theta_{w, \text { onshore }}=-\theta_{w, \text { offshore }}=<\left|\theta_{w}(t)\right|>=\frac{1}{4} \frac{f_{w} U_{w}^{2}}{(s-1) g d_{50}}
$$

where $<\left|\theta_{w}(t)\right|>=\theta_{w, m}$ is the time-averaged absolute value of the instantaneous Shields parameter.

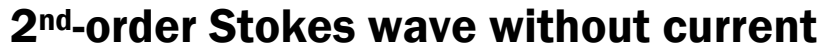

Assuming a wave velocity profile following Stokes $2^{\text {nd }}-$ order wave theory, (i.e., $u_{w}(t)=U_{w}\left(\cos \omega t+r_{w} \cos 2 \omega t\right)$, where $r_{w}$ is the wave asymmetry), the mean Shields parameters become (see Camenen 2002):

$$
\begin{aligned}
& \theta_{w, \text { onshore }}=\frac{1}{2} \frac{f_{w}}{(s-1) g d_{50}} \frac{U_{w}^{2}}{2}\left(1+r_{w}^{2}+\frac{13}{6} r_{w} \frac{\sin a_{c}}{a_{c}}+\frac{1}{6} \frac{\sin 2 a_{c}}{2 a_{c}}\right) \\
& \theta_{w, \text { ofshore }}=\frac{1}{2} \frac{f_{w}}{(s-1) g d_{50}} \frac{U_{w}^{2}}{2}\left(-1-r_{w}^{2}+\frac{13}{6} r_{w} \frac{\sin a_{t}}{a_{t}}-\frac{1}{6} \frac{\sin 2 a_{t}}{2 a_{t}}\right)
\end{aligned}
$$

where $a_{c}=\pi T_{w c} / T_{w}$ and $a_{t}=\pi T_{w t} / T_{w}=\pi-a_{c}$.

Moreover, due to the asymmetry, $T_{w c}$ and $T_{w t}$ are no longer equal to $T_{w} / 2$ : 


$$
\begin{aligned}
& T_{w c}=\frac{T_{w}}{\pi} \arccos \left(\frac{\sqrt{\Delta U}-1}{4 r_{w}}\right) \\
& T_{w t}=T_{w}-T_{w c}
\end{aligned}
$$

where $\Delta U=1+8 r_{w}^{2}$ and $r_{w} \neq 0$.

\section{Sinusoidal wave with current}

The interaction between a steady current and a wave with a sinusoidal velocity variation yields the following relationships for the mean Shields parameter in the onshore and offshore direction, respectively:

$$
\begin{aligned}
& \theta_{c w, \text { onshore }}=\frac{1}{2} \frac{f_{c w}}{(s-1) g d_{50}}\left(\left(U_{c} \cos \varphi\right)^{2}+\frac{U_{w}^{2}}{2}+\frac{3}{2} U_{c} \cos \varphi U_{w} \frac{\sin a_{c}}{a_{c}}\right) \\
& \theta_{c w, \text { offhore }}=\frac{1}{2} \frac{f_{c w}}{(s-1) g d_{50}}\left(-\left(U_{c} \cos \varphi\right)^{2}-\frac{U_{w}^{2}}{2}+\frac{3}{2} U_{c} \cos \varphi U_{w} \frac{\sin a_{t}}{a_{t}}\right)
\end{aligned}
$$

As for an asymmetric wave velocity profile, $T_{w c}$ and $T_{w t}$ are no longer equal to $T_{w} / 2$ because of the current effect:

$$
\begin{aligned}
& T_{w c}=\frac{T_{w}}{\pi} \arccos \left(-r_{o}\right) \\
& T_{w t}=T_{w}-T_{w c}
\end{aligned}
$$

where $r_{o}=U_{c} \cos \varphi / U_{w}$ and $T_{w c}=T_{w}$ if $r_{o} \geq 1$ and $T_{w c}=0$ if $r_{o} \leq-1$.

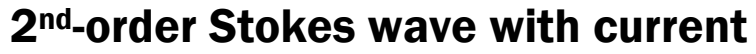

Assuming a wave velocity variation following $2^{\text {nd }}$-order Stokes wave theory (i.e., $u_{w}(t)=U_{w}\left(\cos (\omega t)+r_{w} \cos (2 \omega t)\right)$, where $r_{w}$ is the wave asymmetry), the mean Shields parameters become (see Camenen 2002): 


$$
\begin{aligned}
& \theta_{c w, \text { onshore }}=\frac{1}{2} \frac{f_{c w}}{(s-1) g d_{50}}\left(\begin{array}{l}
\left(U_{c} \cos \mathrm{j}\right)^{2}+\frac{U_{w}^{2}}{2}\left(1+r_{w}^{2}+\frac{13}{6} r_{w} \frac{\sin a_{c}}{a_{c}}+\frac{1}{6} \frac{\sin 2 a_{c}}{2 a_{c}}\right) \\
+U_{c} \cos U_{w}\left(\frac{19}{12} \frac{\sin a_{c}}{a_{c}}+\frac{3}{2} r_{w} \frac{\sin 2 a_{c}}{2 a_{c}}\right)
\end{array}\right) \\
& \theta_{c w, \text { offshore }}=\frac{1}{2} \frac{f_{c w}}{(s-1) g d_{50}}\left(\begin{array}{l}
-\left(U_{c} \cos \right)^{2}-\frac{U_{w}^{2}}{2}\left(1+r_{w}^{2}-\frac{13}{6} r_{w} \frac{\sin a_{t}}{a_{t}}+\frac{1}{6} \frac{\sin 2 a_{t}}{2 a_{t}}\right) \\
+U_{c} \cos U_{w}\left(\frac{19}{12} \frac{\sin a_{t}}{a_{t}}-\frac{3}{2} r_{w} \frac{\sin 2 a_{t}}{2 a_{t}}\right)
\end{array}\right)
\end{aligned}
$$

The periods $T_{w c}$ and $T_{w t}$ are calculated using Equation B3 with $\Delta U=1-8\left(r_{o}-r_{w}\right) r_{w}$. When $r_{w} \leq 0.25, T_{w c}=T_{w}$ if $r_{o} \geq 1-r_{w}$ and $T_{w c}=0$ if $r_{o} \leq-1-r_{w}$.

If $r_{w}>0.25$, the $2^{\text {nd }}$ order Stokes wave will produce a "hump" where the minimum value should be observed, and some modifications are necessary for these extreme cases:

$$
\begin{aligned}
& T_{w c}=\frac{T_{w}}{\pi}\left(\pi+\arccos \left(\frac{\sqrt{\Delta U}-1}{4 r_{w}}\right)-\arccos \left(\frac{-\sqrt{\Delta U}-1}{4 r_{w}}\right)\right) \\
& T_{w t}=T_{w}-T_{w c}
\end{aligned}
$$




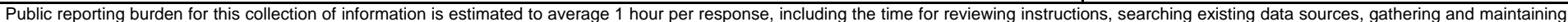

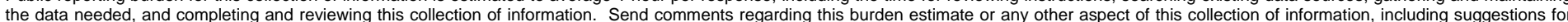

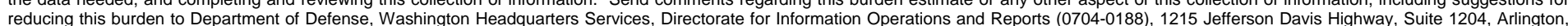

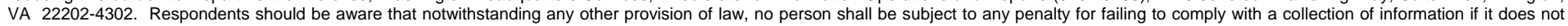
display a currently valid OMB control number. PLEASE DO NOT RETURN YOUR FORM TO THE ABOVE ADDRESS.
1. REPORT DATE (DD-MM-YYYY) September 2007 2. REPORT TYPE Final report 4. TITLE AND SUBTITLE

\section{A Unified Sediment Transport Formulation for Coastal Inlet Application}

\section{AUTHOR(S)}

Benoît Camenen and Magnus Larson
3. DATES COVERED (From - To)

5a. CONTRACT NUMBER

5b. GRANT NUMBER

5c. PROGRAM ELEMENT NUMBER

5d. PROJECT NUMBER

5e. TASK NUMBER

5f. WORK UNIT NUMBER

8. PERFORMING ORGANIZATION REPORT NUMBER

ERDC/CHL CR-07-1

10. SPONSOR/MONITOR'S ACRONYM(S)

Headquarters, U.S. Army Corps of Engineers

Washington, DC 20314-1000
11. SPONSOR/MONITOR'S REPORT NUMBER(S)

\section{DISTRIBUTION / AVAILABILITY STATEMENT}

Approved for public release; distribution is unlimited.

\section{SUPPLEMENTARY NOTES}

\section{ABSTRACT}

The Coastal Inlets Research Program (CIRP) is developing predictive numerical models for simulating the waves, currents, sediment transport, and morphology change at and around coastal inlets. Water motion at a coastal inlet is a combination of quasi-steady currents such as river flow, tidal current, wind-generated current, and seiching, and of oscillatory flows generated by surface waves. Waves can also create quasi-steady currents, and the waves can be breaking or non-breaking, greatly changing potential for sediment transport. These flows act in arbitrary combinations with different magnitudes and directions to mobilize and transport sediment. Reliable prediction of morphology change requires accurate predictive formulas for sediment transport rates that smoothly match in the various regimes of water motion. This report describes results of a research effort conducted to develop unified sediment trans $\neg$ port rate predictive formulas for application in the coastal inlet environ $\neg$ ment. The formulas were calibrated with a wide range of available measurements compiled from the laboratory and field and then imple $\neg$ mented in the CIRP's Coastal Modeling System.

(Continued)

\section{SUBJECT TERMS}

Bed load transport

Coastal sediment transport

16. SECURITY CLASSIFICATION OF:

\section{a. REPORT}

UNCLASSIFIED
Phase lag

Sheet flow

b. ABSTRACT
UNCLASSIFIED

c. THIS PAGE

UNCLASSIFIED
Suspended load transport

Transport by current and waves

\section{LIMITATION OF ABSTRACT \\ 18. NUMBER OF PAGES PERSON}

247

\section{9b. TELEPHONE NUMBER (include} area code) 


\section{7. (Concluded)}

Cemagref Lyon

3 Bis Quai Chauveau

CP 220, 69336 Lyon, CEDEX 09, France;

Department of Water Resources Engineering, Lund University

Box 118, 22100 Lund, Sweden;

Coastal and Hydraulics Laboratory

U.S. Army Engineer Research and Development Center

3909 Halls Ferry Road, Vicksburg, MS 39180-6199

\section{4. (Concluded)}

Emphasis of the study was on reliable predictions over a wide range of input conditions. All relevant physical processes were incorporated to obtain greatest generality, including: (1) bed load and suspended load, (2) waves and currents, (3) breaking and non-breaking waves, (4) bottom slope, (5) initiation of motion, (6) asymmetric wave velocity, and (7) arbitrary angle between waves and current. A large database on sediment transport measurements made in the laboratory and the field was compiled to test different aspects of the formulation over the widest possible range of conditions. Other phenomena or mechanisms may also be of importance, such as the phase lag between water and sediment motion or the influence of bed forms. Modifications to the general formulation are derived to take these phenomena into account. The performance of the new transport formulation was compared to several popular existing predictive formulas, and the new formulation yielded the overall best predictions among the formulas investigated. Results of this report are thus considered to represent a significant and operational step toward a unified formulation for sediment transport at coastal inlets and the nearshore where transport of non-cohesive sediment is common. 